WSRC-TR-2002-00319 Rev. 0

SRT-RPP-2002-0017]

\title{
Preliminary Modeling Results of Evaporated Tc-Eluate Physical Properties
}

SAYANNAH RIVER TECHNOLOGY CENTER

Publication Date: October, 2002

Westinghouse Sayannah River Company

Savannah River Site

Aiken, SC. 29808

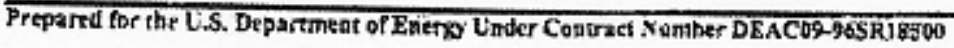

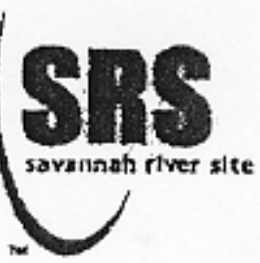


This document was prepared in conjunction with work accomplished under Contract No. DE-AC09-96SR18500 with the U. S. Department of Energy.

\section{DISCLAIMER}

This report was prepared as an account of work sponsored by an agency of the United States Government. Neither the United States Government nor any agency thereof, nor any of their employees, makes any warranty, express or implied, or assumes any legal liability or responsibility for the accuracy, completeness, or usefulness of any information, apparatus, product or process disclosed, or represents that its use would not infringe privately owned rights. Reference herein to any specific commercial product, process or service by trade name, trademark, manufacturer, or otherwise does not necessarily constitute or imply its endorsement, recommendation, or favoring by the United States Government or any agency thereof. The views and opinions of authors expressed herein do not necessarily state or reflect those of the United States Government or any agency thereof.

This report has been reproduced directly from the best available copy.

Available for sale to the public, in paper, from: U.S. Department of Commerce, National Technical Information Service, 5285 Port Royal Road, Springfield, VA 22161, phone: (800) 553-6847, fax: (703) 605-6900

email: orders@ntis.fedworld.gov

online ordering: http://www.ntis.gov/help/index.asp

Available electronically at http://www.osti.gov/bridge

Available for a processing fee to U.S. Department of Energy and its contractors, in paper, from: U.S. Department of Energy, Office of Scientific and Technical Information, P.O. Box 62, Oak Ridge, TN 37831-0062,

phone: (865)576-8401,

fax: (865)576-5728

email: $\underline{\text { reports@ adonis.osti.gov }}$ 
WSRC-TR-2002-00319 Rev. 0

SRT-RPP-2002-00171

KEYWORDS:

Hanford River Protection Project

Retention:

Permanent

Key WTP R\&T References:

Test Specification Document: 24590-WTP-TSP-RT-01-008Rev.0

Task Technical Plan Document:

WSCR-TR-2002-00408, Rev. 0

Test Scoping Statement:

S79

\section{Preliminary Modeling Results of Evaporated Tc-Eluate Physical Properties}

SAVANNAH RIVER TECHNOLOGY CENTER

C. D. Barnes

T. B. Edwards

A. S. Choi

Publication Date: August, 2002

Westinghouse Savannah River Company

Savannah River Site

Aiken, SC 29808

Prepared for the U.S. Department of Energy Under Contract Number DEAC09-96SR18500

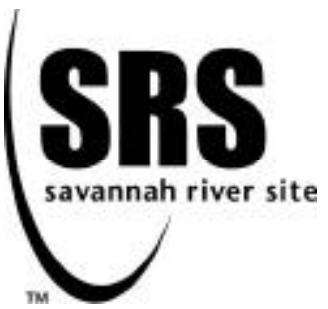




\section{Table of Contents}

1 Summary of Testing ............................................................................................1

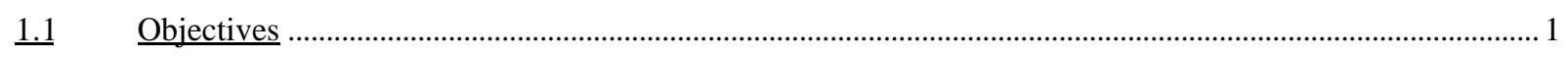

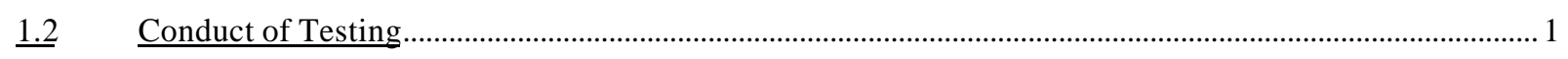

$1.3 \quad$ Results and Performance Against Objectives ..........................................................................................

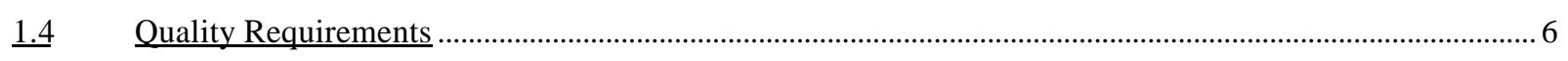

$1.5 \quad$ Issues

$\underline{2}$ Introduction and Background.......................................................................8

$\underline{3}$ Discussion....................................................................................................................9

3.1 Determination of Significant Eluate Species ……....................................................................................

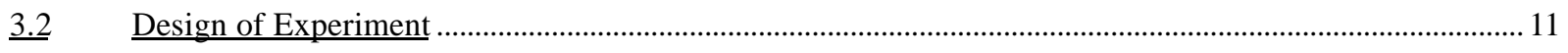

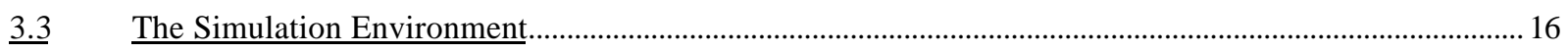

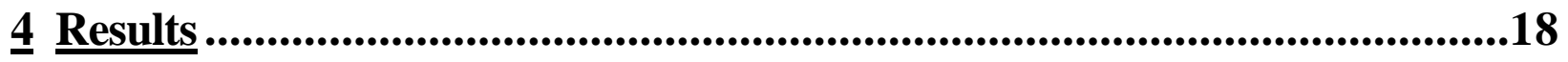

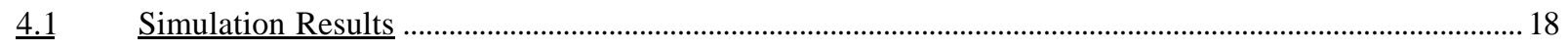

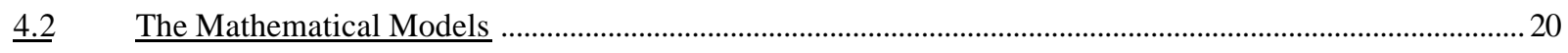

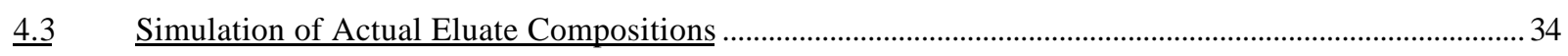

$\underline{5}$ Dynamic Simulation............................................................................38

$\underline{6}$ Conclusion/Summary .........................................................................................41

$\underline{7}$ References ...................................................................................................

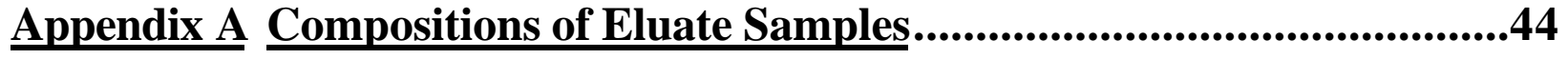

Appendix B Sample Calculation ...............................................................................45

Appendix C Quality Assurance; Verification of Excel Macros and Perl

Script ........................................................................................................46

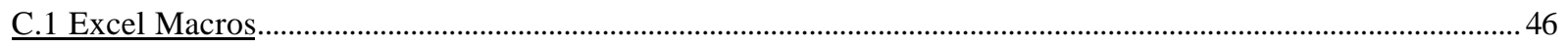

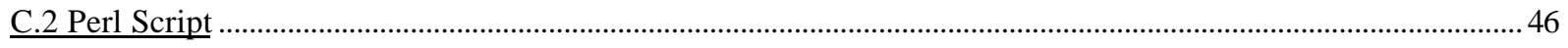

C.3 Example Verification .............................................................................................................................. 46

Appendix D Design of Experiment..................................................................51

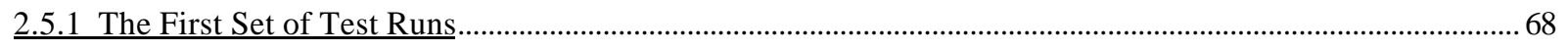

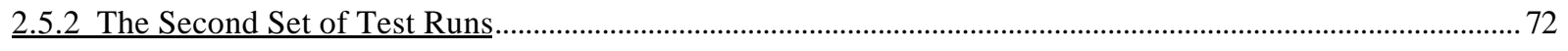

2.5.3 The Third and Final Set of Test Runs ……………….............................................................................. 74

2.5.4 The Complete Test Matrix ............................................................................................................................... 


\section{List of Figures}

Figure 3-1. OLI/ESP Tc Eluate Evaporation Flowsheet ……………................................................................17

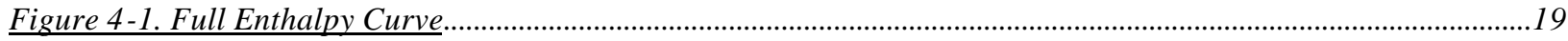

Figure 4-2. Portion of Enthalpy Curve used for Heat Capacity Determination ..........................................................19

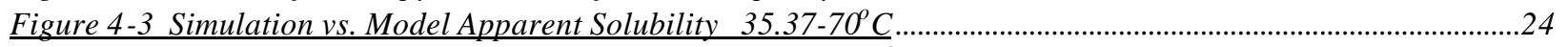

Figure 4-4 Simulation vs. Model Water Mass Fraction 35.37-70 C........................................................................24

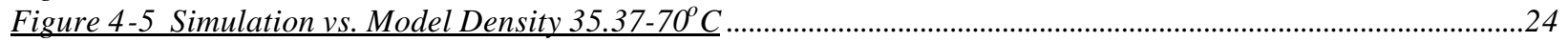

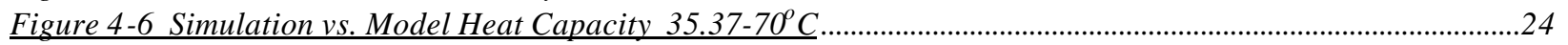

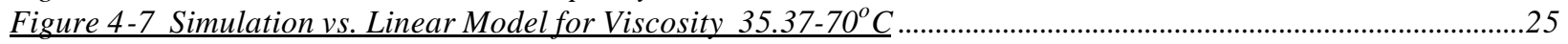

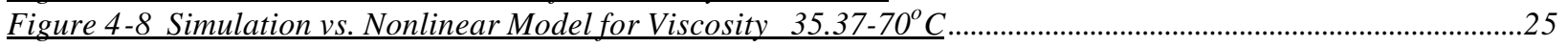

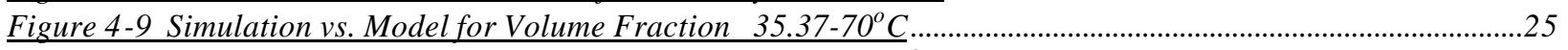

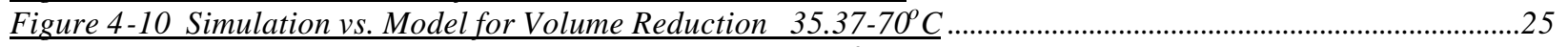

Figure 4-11 Simulation vs. Model Apparent Solubility 20-35.37 C...........................................................................28

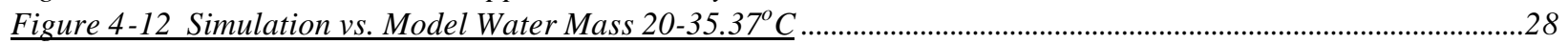

Figure 4-13 Simulation vs. Model Density 20-35.37 C .....................................................................................28

Figure 4-14 Simulation vs. Model Heat Capacity 20-35.37 C..............................................................................28

Figure 4-15 Simulation vs. Linear Model for Viscosity 20-35.37 C .....................................................................29

Figure 4-16 Simulation vs. Nonlinear Model for Viscosity 20-35.37 C..................................................................29

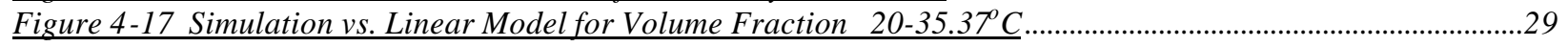

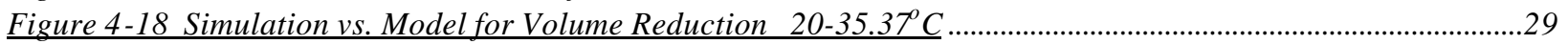

Figure 4-19 Simulation vs. Model Apparent Solubility 20-35.37 $7^{\circ}$ (simulation of actual samples)...........................35

Figure 4-20 Simulation vs. Model Water Mass 20-35.37 $\mathrm{C}$ (simulation of actual samples) ........................................35

Figure 4-21 Simulation vs. Model Density 20-35.37 C (simulation of actual samples) ...........................................36

Figure 4-22 Simulation vs. Nonlinear Model Viscosity 20-35.37 C (simulation of actual samples) ..............................36

Figure 4-23 Simulation vs. Model for Volume Reduction $20-35.37^{\circ} \mathrm{C}$ (simulation of actual samples) .......................36

Figure 4-24 Simulation vs. Model Apparent Solubility $35.37-70^{\circ} \mathrm{C}$ (simulation of actual samples)..........................36

Figure 4-25 Simulation vs. Model Water Mass Fraction 35.37-70 ${ }^{\circ} \mathrm{C}$ (simulation of actual samples) .........................37

Figure 4-26 Simulation vs. Model Density 35.37-70 $\mathrm{C}$ (simulation of actual samples) .................................................37

Figure 4-27 Simulation vs. Nonlinear Model Viscosity 35.37-70 ${ }^{\circ} \mathrm{C}$ (simulation of actual samples) ..........................37

Figure 4-28 Simulation vs. Model Volume Reduction $35.37-70^{\circ} \mathrm{C}$ (simulation of actual samples)................................37 


\section{List of Tables}

Table 1-1 Mass Fraction Ranges for which Models are Valid..................................................................................

Table 3-1 Mass Fraction Ranges for which Models are Valid............................................................................... 9

Table 3-2 Composition of Radioactive Tc Eluate Samples above Minimum Detection Limits.........................................10

Table 3-3 Design Point Compositions Simulated using OLI/ESP ……….................................................................. 14

Table 3-4 Effect of Feed to Air In-leakage Flow Ratio on Product Composition.......................................................18

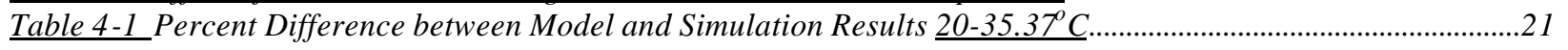

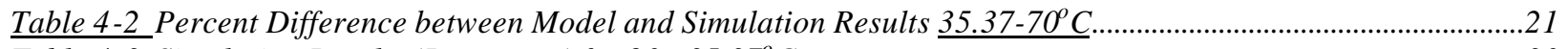

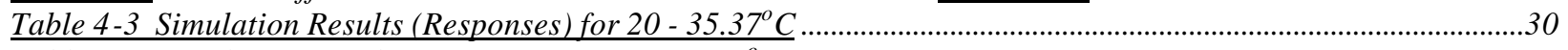

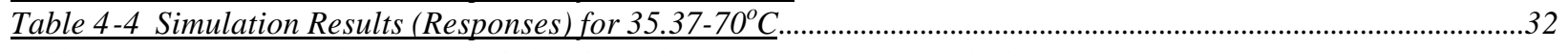

Table 4-5 Comparison between Model and Simulation Results of Actual Eluate Compositions .................................34

Table A-1 Anion Mass Fractions of Actual Tc Eluate Samples...................................................................................4

Table A-2 Composition of Radioactive Tc Eluate Samples Above Minimum Detection Limits.....................................44

Table B-3 Mass Fraction Ranges for which Models are Valid ..................................................................................45

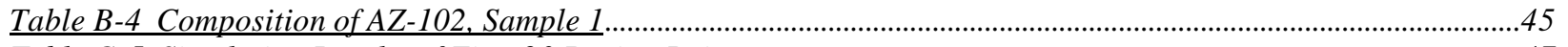

Table C-5 Simulation Results of First 20 Design Points ..........................................................................................47

Table C-6 Enthalpies of First 4 Design Points .....................................................................................................48

Table C-7 Portion of OLI/ESP Simulation Output File for Design Point 1 Showing Eluate Stream ...........................48

Table C-8 Portion of OLI/ESP Simulation Output File .................................................................................................49 


\section{Summary of Testing}

\subsection{Objectives}

The original scope of this task was to develop mathematical expressions for the apparent solubility, density and heat capacity of concentrated technetium eluate solutions as a function of temperature and concentrations of significant analytes present in the as-received eluate feeds. The task scope was later expanded to develop additional correlations for viscosity and the volume reduction factor that can be achieved at $80 \%$ of the prescribed evaporation endpoint. The apparent solubility is defined in this report as either the saturation point of any major salt species present or the point at which the sum of all insoluble solids formed from the remaining minor constituents adds up to $1 \mathrm{wt} \%$ of the solution, whichever occurs first. This definition of solubility captures the features important to the operation of the evaporator, this is, small amounts of insoluble solids are acceptable during operation of the evaporation and generally do not increase significantly during evaporation, where as large amounts of a major salt (a relatively high concentration of the salt's ion constituents are present in solution) form when the solution is concentrated beyond the salt's solubility point. The necessary data to develop such correlations were calculated by the steady state computer simulation of technetium eluate evaporation using a statistically designed matrix of test solutions as the feed. The resulting correlations are to be used to support the design and operation of the technetium eluate evaporator for the Hanford River Protection Project (RPP) Waste Treatment Plant (WTP). The acceptance criterion for these correlations was set to predict measured physical properties within an error of $\pm 15 \%$ with a confidence of 1 -sigma ${ }^{1}$. The scope was further expanded during this task to include a dynamic computer simulation in addition to the steady state simulations to address the concern over the potential of forming gibbsite during the initial phase of evaporation when the solution $\mathrm{pH}$ in the pot is at its minimum.

\subsection{Conduct of Testing}

The significant eluate species and their concentration ranges were determined from the results of previous ion exchange lab experiments using actual tank samples. A design of experiments [2] was done based on these concentration ranges, producing a matrix of 132 design points. The simulation results of up to 88 of these design points were used to fit trial mathematical expressions for the physical properties of the eluate. The simulation results of the remaining 41 design points were used for comparison with the model predictions as a way of verifying the model since no experimental data on actual eluate evaporation are currently available (the models will also be verified against results from the evaporation of simulant Tc eluate currently in progress). Simulations of the eluate evaporation were done using the OLI Environmental Software Program (OLI/ESP) version 6.5 using a simple OLI/ESP chemistry model derived from the significant species to represent the eluate. Excel macros were used to automate the execution of the simulations.

The evaporation was simulated as a flash calculation at a fixed temperature of $70^{\circ} \mathrm{C}$ and at the boiling-point pressure of the concentrated eluate. The evaporation endpoint, for apparent solubility (and water mass fraction) only, was defined to be the eluate concentrated to $1.0 \mathrm{wt} \%$ insoluble solids, or the first appearance of a major salt, which ever occurred first, at some specified temperature between $20-70^{\circ} \mathrm{C}$ (not necessarily the evaporator operating temperature). The evaporation endpoint for all other physical properties was defined to be the eluate concentrated to $0.8 \mathrm{wt} \%$ insoluble solids, or a major salt equilibrium constant $\left(\mathrm{K}_{\text {major salt }}\right)$ equal to $80 \%$ of its equilibrium constant at saturation $\left(\mathrm{K}_{\text {major salt }}=0.8 * \mathrm{~K}_{\text {sp-major salt }}\right)$. The physical

\footnotetext{
${ }^{1}$ A confidence of 1-sigma is defined such that the value predicted by the model has a $67 \%$ chance of being within the stated error ( $15 \%$ in this case) of the actual value.
} 


\section{WSRC-TR-2002-00319 Rev. 0 \\ SRT-RPP-2002-00171}

properties calculated by OLI/ESP were fit to the trial expressions (polynomials, $1^{\text {st }}$ and/or $2^{\text {nd }}$ order in composition, with and without temperature terms) by $\mathrm{JMP}^{\circledR}$ version 4.05 statistical software [15] using least squares linear regression. In addition to the linear fits, a non-linear fit was done for viscosity to a well-known form for viscosity (Vogel, a function of temperature only, and generally used for pure fluids) using the non-linear platform in JMP ${ }^{\circledR}$.

OLI/ESP (version 6.5) is not capable of determining heat capacity directly. Hence, the heat capacity was calculated using enthalpy vs. temperature plots created from a series of simulations at the endpoint compositions by varying the temperature $\pm 2{ }^{\circ} \mathrm{C}$, in increments of $0.2^{\circ} \mathrm{C}$ about the endpoint temperature.

The original temperature range of $20-70^{\circ} \mathrm{C}$ was divided into two ranges $\left(20-35.37^{\circ} \mathrm{C}\right.$ and $\left.35.37-70^{\circ} \mathrm{C}\right)$ and separate physical property expressions were derived for each of the temperature ranges. This was necessary because of the opposing solubility behavior among the three hydrated forms of the sodium carbonate salts, considered to be major salts. This is more fully described in the discussion (Section 3) of this report. Attempts to capture these opposing behaviors in a single expression over the entire temperature range introduced significant error as compared to the separate expressions derived for each of the two temperature ranges.

\subsection{Results and Performance Against Objectives}

\section{Dynamic Simulation}

The results of dynamic simulation showed that in the case of AZ-102 technetium eluate evaporation up to $30 \%$ of total aluminum fed could remain undissolved in the pot as gibbsite. However, the formation of gibbsite would not be an operational issue due to its low concentration. Instead, the target evaporation endpoint of $1.0 \mathrm{wt} \%$ insoluble solids in the pot would be reached due to formation of sodium oxalate crystals, and the maximum volume reduction factor that can be achieved at that endpoint is $65 \mathrm{X}$. Furthermore, the likelihood of forming any major salts such as $\mathrm{NaNO}_{3}, \mathrm{Na}_{2} \mathrm{CO}_{3} \cdot \mathrm{xH}_{2} \mathrm{O}$ (where $\mathrm{x}$ is 1 , 7, or 10 for the mono-, hepta-, or deca-hydrated salt) or more importantly $\mathrm{NaTcO}_{4}$ and $\mathrm{KTcO}_{4}$, out of the AZ102 technetium eluate solution would still be remote even at 100X volume reduction.

\section{Physical Property Models}

Model predictions were compared to OLI/ESP simulations for the 41 test points, as well as OLI/ESP evaporation simulations using the compositions of actual Tc eluate samples. Simulations of the eluate samples used an expanded OLI/ESP chemistry model based on all species found in the actual eluate samples above their minimum detection limits.

The success of a model was measured by the distribution of the percent differences between the model predictions and simulation results ((simulation-model)/model*100) for the 41 test points. The error of the mathematical model is defined to be standard deviation of the percent differences (giving a confidence of 1-sigma).

The equations 1-5, and 7-11 below are in terms of the mass fractions of the anions relative to the total mass of all eight anions, where $[\mathrm{AlO} 2]$ is the aluminate mass fraction, $\left[\mathrm{C}_{2} \mathrm{O}_{4}\right]$ the oxalate mass fraction, $\left[\mathrm{CO}_{3}\right]$ the carbonate mass fraction, $\left[\mathrm{NO}_{2}\right]$ the nitrite mass fraction, $\left[\mathrm{NO}_{3}\right]$ the nitrate mass fraction, $[\mathrm{OH}]$ the hydroxide mass fraction, $\left[\mathrm{SO}_{4}\right]$ the sulfate mass fraction, and $\left[\mathrm{TcO}_{4}\right]$ the pertechnetate mass fraction. The non-linear viscosity equation (6) is a function of temperature only, and the corresponding equation (12) for the lower temperature range is also a function of density. Equations (1) and (9) for apparent solubility and equations (2) and (10) for water mass fraction correspond to an eluate concentrated to $1.0 \mathrm{wt} \%$ insoluble solids, or when a major salt begins to precipitate (i.e. its saturation). The remaining equations, 3-8 and 9-16 (the other physical properties), correspond to an eluate concentrated to $0.8 \mathrm{wt} \%$ insoluble solids or when the equilibrium constant of a major salt ( $\left.\mathrm{K}_{\text {major salt }}\right)$ is equal to 0.80 times its solubility product for the mixture 
$\left(\mathrm{K}_{\text {major salt }}=0.8 * \mathrm{~K}_{\mathrm{sp} \text {-major salt }}\right)$. The anion mass fraction ranges which were simulated, and for which equations 1-12 are valid, are listed in Table 1-1. Equations 1-6 are valid for temperatures from $20-35.37^{\circ} \mathrm{C}$, and equations 6-12 are valid for temperatures from $35.37-70^{\circ} \mathrm{C}$.

Table 1-1 Mass Fraction Ranges for which Models are Valid

\begin{tabular}{|c|c|c|c|c|c|c|c|c|}
\hline & $\mathrm{AlO}_{2}$ & $\mathrm{C}_{2} \mathrm{O}_{4}$ & $\mathrm{CO}_{3}$ & $\mathrm{NO}_{2}$ & $\mathrm{NO}_{3}$ & $\mathrm{OH}$ & $\mathrm{SO}_{4}$ & $\mathrm{TcO}_{4}$ \\
\hline $\mathrm{Min}$ & 0.00750 & 0.00001 & 0.16500 & 0.03900 & 0.10500 & 0.01600 & 0.000001 & 0.00042 \\
\hline Max & 0.03000 & 0.02050 & 0.80500 & 0.36000 & 0.73500 & 0.04100 & 0.19500 & 0.02150 \\
\hline
\end{tabular}

weight, and are relative to the total mass of the species listed in the table.

With the exception of the linear fit for viscosity, all trial expressions derived for the $35.37-70^{\circ} \mathrm{C}$ range were well within acceptance criteria of a $15 \%$ error with a confidence of 1-sigma, including the non-linear form for viscosity. Unfortunately, the trial expressions for the $20-35.37^{\circ} \mathrm{C}$ range, with the exception of density, were significantly outside the same acceptance criteria, due largely to the complicated nature of the precipitating species in the lower temperature range.

It should be noted that, with the exception of the non-linear viscosity model, the trial expressions which included temperature terms did not perform any better than those presented here, which are functions of composition only. This is not to suggest that the physical properties are independent of temperature, but that the accuracy of the models is broad relative to the variations in the physical properties for composition and temperature ranges considered here. That is, the error in the model prediction is on the order of, or greater than, the variations in the physical properties with respect to temperature.

\section{Expressions valid for $35.37-70^{\circ} \mathrm{C}$}

The apparent solubility ( $1 \mathrm{wt} \%$ insoluble solids or precipitation of a major salt) is given as:

$$
\begin{aligned}
& \text { solubility at endpoint conditions }\left(\mathrm{g} \text { solids/kg } \mathrm{H}_{2} \mathrm{O}\right) \\
& \begin{aligned}
\mathbf{7 2 8} *\left[\mathrm{AlO}_{2}\right]-2,510 *\left[\mathrm{CO}_{4}\right]+492 *[\mathrm{CO}]+554 *[\mathrm{NO}]+1,070 *[\mathrm{NO}] \\
\quad+\mathbf{2 , 7 7 0} *[\mathrm{OH}]+827 *[\mathrm{SQ}]+733 *[\mathrm{Tc} \Theta]
\end{aligned}
\end{aligned}
$$

having a mean of $4.7 \%$ and a standard deviation of $9.9 \%$. The concentrations are in terms of the anion mass fraction relative to the total mass of the anions.

The water mass fraction (WMF) is given as:

$$
\begin{aligned}
& \text { WMF at endpoint conditions }\left(\mathrm{g} \mathrm{H}_{2} \mathrm{O} / \mathrm{g}\right. \text { solution) } \\
& =0.498 *\left[\mathrm{AlO}_{2}\right]+1.59 *\left[\mathrm{C}_{2} \mathrm{O}_{4}\right]+0.662 *\left[\mathrm{CO}_{3}\right]+0.624 *\left[\mathrm{NO}_{2}\right]+0.477 *\left[\mathrm{NO}_{3}\right] \\
& \quad-0.03099 *[\mathrm{OH}]+0.575 *\left[\mathrm{SO}_{4}\right]+0.608 *\left[\mathrm{TcO}_{4}\right]
\end{aligned}
$$

having a mean of $-2.60 \%$ and a standard deviation of $3.93 \%$. The concentrations are in terms of the anion mass fraction relative to the total mass of the anions. 
The density is given as:

$$
\begin{aligned}
& \text { density at endpoint conditions }(\mathrm{g} / \mathrm{L}) \\
& =1,920 *[\mathrm{AlO}]+1,150 *\left[\mathrm{C}_{2} \mathrm{O}_{4}\right]+1,260 *[\mathrm{CO}]+1,210 *[\mathrm{NO}] \\
& \quad+1,300 *[\mathrm{NO}]+2,470 *[\mathrm{OH}]+1,180 *\left[\mathrm{SO}_{4}\right]+1,060 *[\mathrm{TcO}]
\end{aligned}
$$

having a mean of $0.33 \%$ and a standard deviation of $2.09 \%$. The concentrations are in terms of the anion mass fraction relative to the total mass of the anions.

The heat capacity is given as:

$$
\begin{aligned}
& \text { heat capacity at endpoint conditions }\left(\mathrm{cal} / \mathrm{g} /{ }^{\mathrm{o}} \mathrm{C}\right) \\
& =0.504 *[\mathrm{AlO}]+\mathbf{2 . 7 0} *\left[\mathrm{GO}_{4}\right]+0.656 *[\mathrm{CO}]+0.736 *[\mathrm{NO}] \\
& +0.559 *[\mathrm{NO}]-1.06 *[\mathrm{OH}]+0.667 *[\mathrm{SO}]+1.23 *[\mathrm{TcO}]
\end{aligned}
$$

having a mean of $1.16 \%$ and a standard deviation of $4.32 \%$. The concentrations are in terms of the anion mass fraction relative to the total mass of the anions.

The viscosity is given as:

$$
\begin{aligned}
& \text { viscosity at endpoint conditions }(\mathrm{cP}) \\
&= 28.5 *\left[\mathrm{AlO}_{3}\right]+37.2 *\left[\mathrm{C}_{2} \mathrm{O}_{4}\right]+3.02 *\left[\mathrm{CO}_{3}\right]+2.41 *\left[\mathrm{NO}_{2}\right] \\
&+1.18 *\left[\mathrm{NO}_{3}\right]-\mathbf{7 . 1 4} *[\mathrm{OH}]+\mathbf{1 . 0 0} *[\mathrm{SO}]+\mathbf{2 2 . 9} *[\mathrm{TcO}]
\end{aligned}
$$

having a mean of $6.62 \%$ and a standard deviation of $27.7 \%$. The concentrations are in terms of the anion mass fraction relative to the total mass of the anions. Because of the poor performance of this form of equation for viscosity, the form given by equation (6) below should be used instead.

The Vogel form for viscosity is given as:

$$
\begin{aligned}
& \text { viscosity at endpoint conditions }(\mathrm{cP}) \\
& =\exp \left(\frac{140}{\left(\text { temperature }\left({ }^{\circ} \mathrm{C}\right)+20\right)}-100\right)
\end{aligned}
$$

having a mean of $0.07 \%$ and a standard deviation of $8.62 \%$, and is a function of temperature only. This form should be used in lieu of equation (5).

The volume fraction is given as:

$$
\begin{aligned}
& \text { volume fraction at endpoint conditions } \\
& \text { (concentrated eluate volume / evaporator feed volume at } 20^{\circ} \mathrm{C} \text { and } 1 \mathrm{wt} \% \text { total solids) } \\
& =0.0117 *\left[\mathrm{AlO}_{2}\right]+\mathbf{0 . 0 3 3 3} *\left[\mathrm{CO}_{4}\right]+0.0145 *\left[\mathrm{CO}_{3}\right]+0.0118 *[\mathrm{NO}] \\
& \quad+\mathbf{0 . 0 0 8 0 2} *\left[\mathrm{NO}_{3}\right]-0.00946 *[\mathrm{OH}]+\mathbf{0 . 0 1 0 5}^{*}\left[\mathrm{SO}_{4}\right]+0.0156 *[\mathrm{TcO}]
\end{aligned}
$$

having a mean of $4.9 \%$ and a standard deviation of $5.58 \%$. The concentrations are in terms of the anion mass fraction relative to the total mass of the anions. The volume fraction is relative to a feed volume containing $1 \mathrm{wt} \%$ total solids at $20^{\circ} \mathrm{C}$. 
The volume reduction is given as:

volume reduction at endpoint conditions

(evaporator feed volume at $20^{\circ} \mathrm{C}$ and $1 \mathrm{wt} \%$ total solids / concentrated eluate volume)

$=63.7 *\left[\mathrm{AlO}_{2}\right]-86.8 *\left[\mathrm{C}_{2} \mathrm{O}_{4}\right]+67.0 *\left[\mathrm{CO}_{3}\right]+79.0 *[\mathrm{NO}]$

$+117 *\left[\mathrm{NO}_{3}\right]-258 *[\mathrm{OH}]+103 *[\mathrm{SQ}]+58.9 *[\mathrm{TcQ}]$

having a mean of $4.19 \%$ and a standard deviation of $6.36 \%$. The concentrations are in terms of the anion mass fraction relative to the total mass of the anions. The volume reduction is relative to a feed volume containing $1 \mathrm{wt} \%$ total solids at $20^{\circ} \mathrm{C}$.

\section{Expressions valid for $20-35.37^{\circ} \mathrm{C}$}

The apparent solubility (1wt\% insoluble solids or precipitation of a major salt) is given as:

solubility at endpoint conditions ( $\mathrm{g}$ solids/ $\left./ \mathrm{kg} \mathrm{H}_{2} \mathrm{O}\right)$

$$
\begin{aligned}
= & -167,000 *[\mathrm{AlO}]-74,600 *\left[\mathrm{COO}_{4}\right]+12,000 *\left[\mathrm{CO}_{3}\right]+16,300 *[\mathrm{NQ}] \\
& +22,900 *\left[\mathrm{NO}_{3}\right]+48,800 *[\mathrm{OH}]+3,160 *\left[\mathrm{SO}_{4}\right]-173,000 *[\mathrm{TcQ}]
\end{aligned}
$$

having a mean of $21.9 \%$ and a standard deviation of $48 \%$. The concentrations are in terms of the anion mass fraction relative to the total mass of the anions.

The water mass fraction (WMF) is given as:

$$
\begin{aligned}
& \text { WMF at endpoint conditions (grams } \left.\mathrm{H}_{2} \mathrm{O} / \text { grams solution }\right) \\
& \begin{array}{l}
=4.62 * \mathrm{AlO}_{2}+4.20 * \mathrm{C}_{2} \mathrm{O}_{4}+0.516 * \mathrm{CO}_{3}+0.451 * \mathrm{NO}_{2}+0.207 * \mathrm{NO}_{3} \\
\quad-1.58 * \mathrm{OH}+0.668 * \mathrm{SO}_{4}+5.20 * \mathrm{TcO}_{4}
\end{array}
\end{aligned}
$$

having a mean of $-10.5 \%$ and a standard deviation of $18.8 \%$. The concentrations are in terms of the anion mass fraction relative to the total mass of the anions.

The density is given as:

$$
\begin{aligned}
& \text { density at endpoint conditions }(\mathrm{g} / \mathrm{L}) \\
& =-1,520 *[\mathrm{AlO}]+762 *\left[\mathrm{C}_{2} \mathrm{O}_{4}\right]+1,310 *[\mathrm{CO}]+1,490 *[\mathrm{NO}] \\
& \quad+1,620 *[\mathrm{NO}]+2,510 *[\mathrm{OH}]+1,100 *[\mathrm{SO}]-2,670 *[\mathrm{TcO}]
\end{aligned}
$$

having a mean of $5.5 \%$ and a standard deviation of $7.0 \%$. The concentrations are in terms of the anion mass fraction relative to the total mass of the anions.

The heat capacity is given as:

$$
\begin{aligned}
& \text { heat capacity at endpoint conditions }\left(\mathrm{cal} / \mathrm{g} /{ }^{\circ} \mathrm{C}\right) \\
& =3.57 *[\mathrm{AlO}]+3.17 *\left[\mathrm{CO}_{4}\right]+0.687 *[\mathrm{CO}]+0.528 *[\mathrm{NO}] \\
& +0.349 *\left[\mathrm{NO}_{3}\right]-1.62 *[\mathrm{OH}]+0.738 *\left[\mathrm{SO}_{4}\right]+4.76 *\left[\mathrm{TcO}^{-1}\right]
\end{aligned}
$$

having a mean of $-11.5 \%$ and a standard deviation of $13.6 \%$. The concentrations are in terms of the anion mass fraction relative to the total mass of the anions. 
The viscosity is given as:

$$
\begin{aligned}
& \text { viscosity at endpoint conditions }(\mathrm{cP}) \\
& =28.5 *[\mathrm{AlO}]+37.2 *\left[\mathrm{C}_{2} \mathrm{O}_{4}\right]+3.02 *\left[\mathrm{CO}_{3}\right]+2.41 *[\mathrm{NO}] \\
& +1.18 *[\mathrm{NO}]-7.14 *[\mathrm{OH}]+1.00 *\left[\mathrm{SO}_{4}\right]+22.9 *[\mathrm{TcO}]
\end{aligned}
$$

having a mean of $44.5 \%$ and a standard deviation of $68.8 \%$. The concentrations are in terms of the anion mass fraction relative to the total mass of the anions.

The alternative form non-linear form for viscosity (Vogel) is given below:

$$
\begin{aligned}
& \text { viscosity at endpoint conditions }(\mathrm{cP}) \\
& =\exp \left(000174 * \operatorname{density}(\mathrm{g} / \mathrm{L})-\frac{0.0181 * \operatorname{density}(\mathrm{g} / \mathrm{L})}{\left(\operatorname{temperature}\left({ }^{\circ} \mathrm{C}\right)+5\right)}\right)
\end{aligned}
$$

having a mean of $54.8 \%$ and a standard deviation of $65.7 \%$, and is a function of temperature and density only. Note that unlike its corresponding equation (6) for the $35.37-70^{\circ} \mathrm{C}$ range, this equation includes density terms. The measured density of the sample should be used as opposed to that calculated by equation (11).

The volume fraction is given as:

volume fraction at endpoint conditions

(concentrated eluate volume / evaporator feed volume at $20^{\circ} \mathrm{C}$ and $1 \mathrm{wt} \%$ total solids)

$=0.0626 *\left[\mathrm{AlO}_{2}\right]+0.0720 *\left[\mathrm{G}_{4}\right]+0.0239 *\left[\mathrm{CO}_{3}\right]+0.00626 *\left[\mathrm{NO}_{2}\right]$

$+0.00291 *\left[\mathrm{NO}_{3}\right]-0.0808 *[\mathrm{OH}]+0.0149 *[\mathrm{SO}]+0.0649 *\left[\mathrm{TcO}_{4}\right]$

having a mean of $-26 \%$ and a standard deviation of $19 \%$. The concentrations are in terms of the anion mass fraction relative to the total mass of the anions. The volume fraction is relative to a feed volume containing $1 \mathrm{wt} \%$ total solids at $20^{\circ} \mathrm{C}$.

The volume reduction is given as:

volume reduction at endpoint conditions

(evaporator feed volume at $20^{\circ} \mathrm{C}$ and $1 \mathrm{wt} \%$ total solids / concentrated eluate volume)

$=-888 *[\mathrm{AlO}]-438 *\left[\mathrm{GO}_{4}\right]+\mathbf{8 6 . 0} *[\mathrm{CO}]+142 *[\mathrm{NO}]$

$+198 *[\mathrm{NO}]+636 *[\mathrm{OH}]+32.8 *\left[\mathrm{SO}_{4}\right]-1,290 *[\mathrm{TcQ}]$

having a mean of $23 \%$ and a standard deviation of $29 \%$. The concentrations are in terms of the anion mass fraction relative to the total mass of the anions. The volume reduction is relative to a feed volume containing $1 \mathrm{wt} \%$ total solids at $20^{\circ} \mathrm{C}$.

\subsection{Quality Requirements}

Quality requirements pertaining to OLI/ESP software have been addressed in the document "Software Quality Assurance Plan for Hanford RPP-WTP Evaporator Modeling"[14]. OLI/ESP version 6.5 was used with the private databooks gibbsite, newtc, and carbonat, along with the public databook. 


\section{WSRC-TR-2002-00319 Rev. 0}

SRT-RPP-2002-00171

The Excel macros were verified by manually re-running several design points using the OLI/ESP input file generated by the Excel macro to verify the input and output files resulting from automated simulations. All re-run points exactly reproduced the results of the automated simulations.

\subsection{Issues}

All physical properties have mathematical expressions for the $35.37-70^{\circ} \mathrm{C}$ range that fall within error specified by the task plan [1]. However, with the exception of density, all of the expressions for the 20$35.37^{\circ} \mathrm{C}$ range have an error greater (or much greater) than the error specifications. Further work is necessary to reduce these errors.

These equations should be considered preliminary until validated with experimental results using simulant and actual waste samples. 


\section{Introduction and Background}

This report describes Tc eluate evaporation modeling work done as specified in the Task Technical and Quality Assurance Plan for Cesium and Technetium Eluate Physical Property Modeling[1], items A, C and D of section 2.1.1, in support of the Hanford River Protection Project (RPP) Waste Treatment Plant (WTP) project. The task plan describes work to be done for both Cs and Tc eluates; only the Tc work is described here. Modeling work for the Cs portion of the task plan will be issued as a separate report.

Waste currently stored in underground tanks at Hanford is to be pre-treated, then vitrified for permanent storage. Pre-treatment involves the separation of the tank waste into high level waste (HLW) and low activity waste (LAW) streams. The current flow sheet calls for the tank waste to be blended with RPPWTP recycles ${ }^{2}$ and sent first to ultra-filtration (following precipitation of Sr/TRU for envelope C only), and the filtered solids washed and leached prior to storage for eventual treatment and vitrification as HLW. The permeate is to be sent to two sets of ion exchange columns. The first set is for Cs removal, with the lead $\mathrm{Cs}$ column being eluted with $\mathrm{HNO}_{3}$ and the eluate concentrated for processing as HLW. The Cs effluent is to be fed to the second set of ion exchange columns for Tc removal using SuperLig ® 639 resin. The Tc effluent will be concentrated and sent to LAW for treatment and vitrification. The lead Tc column will be eluted with $\left(70^{\circ} \mathrm{C}\right)$ water and the eluate concentrated using a siphon-flow evaporator prior to processing as HLW.

The Savannah River Technology Center (SRTC) has been asked to develop mathematical expressions for the apparent solubility of the Tc eluate concentrated to either $1.0 \mathrm{wt} \%$ insoluble solids or the initial appearance of a major salt, which ever occurs first, as well as expressions for density, heat capacity, viscosity, and volume fraction (concentrated eluate volume $/$ feed volume @ $20^{\circ} \mathrm{C}$ and $1 \mathrm{wt} \%$ total solids) of the eluate concentrated to $0.8 \mathrm{wt} \%$ insoluble solids or when the equilibrium constant of a major salt $\left(\mathrm{K}_{\text {major salt }}\right)$ is equal to 0.80 times its solubility product for the mixture $\left(\mathrm{K}_{\text {major salt }}=0.8 * \mathrm{~K}_{\mathrm{sp} \text {-major salt }}\right)$. These models are to be used in the design and operation of the Tc eluate evaporator. They are functions of the composition of the dilute eluate feed to the evaporator, and, if appropriate, the temperature at which the concentrated eluate will be stored, thereby providing the ability to predict the evaporation endpoint of the eluate. The models were developed using the results of computer experiments, which simulated the evaporation of eluates over a range of compositions and temperatures. The goal was to develop physical property expressions based on the significant eluate species having an error of $15 \%$ or less with a confidence of 1-sigma.

The significant eluate species and their concentration ranges were determined from the analytical results of previous experimental ion exchange work done using radioactive tank samples, and is described in section 3.1. Based on the concentration ranges, a statistical design of experiments was performed which generated a matrix of composition vectors to be used in the computer simulations. A brief explanation of the design of experiments is included in section 3.2, and the full report is included in Appendix D Section 3.3 describes the simulation environment in which the design points were run. Finally, the simulation results, model fits, and comparison of the models with simulations using actual Tc eluate compositions are presented in sections. $4.2,4.2$, and 4.3 respectively.

${ }^{2}$ If the tank waste is less than $5 \mathrm{M} \mathrm{Na}$ then the waste is blended with recycles and then evaporated prior to filtering in the cross flow filters. 


\section{Discussion}

Initially, physical property expressions were developed which were intended to be valid over the entire 20$70^{\circ} \mathrm{C}$ temperature range. However, after reviewing the results it was found that three hydrated forms of sodium carbonate frequently precipitated out, making it necessary to develop two distinct sets physical property expressions, one set for each of two temperature ranges $\left(20-35.37^{\circ} \mathrm{C}\right.$ and $\left.35.37-70^{\circ} \mathrm{C}\right)$ which were based on the temperatures at which these species precipitated. This is fully described in section 4.0, but mentioned here for clarification in this section.

\subsection{Determination of Significant Eluate Species}

Because the eluate composition is somewhat insensitive to the LAW feed composition (due to the selectivity of the ion exchange column), it is believed that a single model for each physical property could be developed which applies to eluate derived from any of the three waste envelopes-A, B, or C (as opposed to a separate model for each envelope). Therefore, data from each of the envelopes is necessary for determination of the significant species and their concentration ranges. Several reports were reviewed for the determination of significant eluate analytes, and are listed below along with an explanation for those that were excluded. The significant species and their corresponding concentration ranges are also discussed and listed in Table 3-1

Table 3-1. Mass Fraction Ranges for which Models are Valid

\begin{tabular}{|c|c|c|c|c|c|c|c|c|}
\hline & $\mathrm{AlO}_{2}$ & $\mathrm{C}_{2} \mathrm{O}_{4}$ & $\mathrm{CO}_{3}$ & $\mathrm{NO}_{2}$ & $\mathrm{NO}_{3}$ & $\mathrm{OH}$ & $\mathrm{SO}_{4}$ & $\mathrm{TcO}_{4}$ \\
\hline $\mathrm{FW}$ & 58.982 & 88.02 & 60.0093 & 46.002 & 62.005 & 17.007 & 96.064 & 162.9039 \\
\hline $\mathrm{Min}$ & 0.00750 & 0.00001 & 0.16500 & 0.03900 & 0.10500 & 0.01600 & 0.000001 & 0.00042 \\
\hline Max & 0.03000 & 0.02050 & 0.80500 & 0.36000 & 0.73500 & 0.04100 & 0.19500 & 0.02150 \\
\hline
\end{tabular}

Significant anions used as variables in the physical property models and their corresponding mass fraction ranges for which the models are valid. The table includes the anion formula weights used to calculate the mass fractions. Mass fractions are relative to the total mass of the anions listed.

The results of eight Tc ion exchange lab experiments using actual tank samples and SuperLig ${ }^{\circledR} 639$ resin were reviewed. Of these, four (at least one from each of the three envelopes A, B, and C) were used to determine the significant eluate analytes and their concentration ranges. They were from Hanford tanks 241-AN-103 [4], 241-AN-105 [5], 241-AN-107 [6], and 241-AZ-102 [7] (the prefix 241 is common to all Hanford tanks and will be dropped for the rest of this report). Although the relative order of Cs and Tc ion exchange columns were reversed in the experiment using the AN-103 sample, the composition of the eluate relative to that of the feed was not appreciably different from that of other experiments. Therefore, the results are believed to be representative for the purpose of determining significant species. The results of the remaining four reports were not used for the following reasons.

The results from the AW-101 sample [8] were excluded because the column was eluted with $\mathrm{HNO}_{3}$ instead of water as required by the current flow sheet. For the two AN-102 samples ("small" and "large" C eluate samples) $[9,10]$ the anion concentrations determined by ion chromatography $\left(\mathrm{NO}_{2}, \mathrm{NO}_{3}, \mathrm{Cl}, \mathrm{PO}_{4}\right.$, etc...) were below the detection limits making the data unsuitable for this purpose. The columns used in the AN107 experiment [11] had been used previously in the AW-101 [8] experiment mentioned above. Analytical results of the AN-107 eluate indicate that the columns may have been contaminated with AW-101 waste at the onset of the experiment. For example, the excess of anions was so large that $\mathrm{Na}$ (the dominant cation) had to be increased by $60 \%$ in order to achieve a charge balance. Also, the $\mathrm{SiO}_{2}$ concentration was well 
beyond its known solubility. Finally, because Tc exists in both the non-pertechnetate and pertechnetate forms in the ion exchange feed, ammonium pertechnetate ( $\mathrm{Tc}$ as ${ }^{95 \mathrm{~m}} \mathrm{Tc}$ ) was as a tracer in both the AW-101 and $\mathrm{AN}-107$ experiments to give an accurate measure of the pertechnetate break-through curves. In spite of this procedure, the concentration of tracer $T c$ in the effluent relative to that in the feed $\left(\mathrm{C}_{\mathrm{eff}} / \mathrm{C}_{\text {feed }} * 100\right)$ in the initial loading phase was measured to be $145 \%$, and dropped only to $125 \%$ after 100 bed volumes had been fed to the column (the total effluent produced was 170 bed volumes). Therefore, it appears the column was contaminated with constituents from the previous run, which were then being eluted by the ion exchange feed.

The data of the four sets of experimental results $[4,5,6,7]$ used to determine the Tc eluate concentration ranges was somewhat incomplete, due in large part to high minimum detection limits for many anions. The following assumptions were used to charge balance the results. AZ-102 was the only one to include a hydroxide concentration, and the value reported appears unrealistically high. It was presumed that the only source of hydroxide in the eluate samples came from the $0.1 \mathrm{M} \mathrm{NaOH}$ used to displace the eluate feed in the column prior to its elution with water, resulting in one column volume of $0.1 \mathrm{M} \mathrm{NaOH}$ to be included as part of the total eluate sample. AZ-102 was also the only one to report an oxalate concentration above the minimum detection limit, the detection limits in the other three reports being so high as to give no reasonable bounds on their concentrations. Both the TOC and oxalate values reported for AZ-102 appeared to be quite high. The TOC value of the corresponding tank characterization report "Chemical Characterization of an Envelope B/D Sample from Hanford Tank 241-AZ-102" [16] was 4.08 times higher than that of an earlier AZ-102 tank characterization report "Tank Characterization Report for Double-Shell Tank 241-AZ-102" [17]. An adjusted (reduced) eluate TOC value was calculated using the ratio of TOC (adjusted based on the relative sodium concentrations) between the two tank characterization reports (4.08). An (reduced) oxalate value was then calculate from this adjusted TOC value using the ratio of carbon from oxalate to TOC from the original - non-adjusted - eluate data. (later characterization of the sample used in the last AZ-102 tank characterization [16] showed measurable levels of other organics, including complexants). The TIC for AN-105 was reduced significantly (about 50\%) to achieve a charge balance. Its concentration was much higher than that of any other eluate sample, and it is know that $\mathrm{CO}_{2}$ from air is taken up in a basic solution (the reduced AN-105 TIC value still defines the upper bounds of the TIC concentration range used for the design matrix). Nitrate and nitrite were below the minimum detection limits for AN-107. Since it is known that $\mathrm{NaNO}_{3}$ competes for sites with the $\mathrm{TcO}_{4}^{-}$anion in the ion exchange resin, a value of $90 \%$ of the minimum detection limit was used for nitrate and nitrite. The remainder of the charge balance for AZ-102, AN-107, and AN-103 was achieved by adjusting the sodium concentration.

Table 3-2 Composition of Radioactive Tc Eluate Samples above Minimum Detection Limits (Charge Balanced)

\begin{tabular}{|c|c|c|c|c|c|c|c|c|}
\hline ICP-AES & charge & AZ-102 Sample 1 & AZ-102 Sample 2 & AN-105 Sample 1 & AN-105 Sample 2 & \begin{tabular}{|c|} 
AN-107 Sample \\
1
\end{tabular} & AN-107 Sample 2 & AN-103 Sample 1 \\
\hline element & & Mole/L & mole/L & mole/L & mole/L & mole/L & mole/L & mole/L \\
\hline $\mathrm{Al}(\mathrm{AlO} 2)$ & -1 & 0.001564006 & 0.001571 & 0.00103 & 0.0008 & 0.000423 & 0.000439 & 0.000284 \\
\hline $\mathrm{B}$ & 3 & 0.000807511 & 0.000802 & 0.000771 & 0.000671 & 0.000303 & 0.000309 & \\
\hline $\mathrm{Ba}$ & 2 & $1.37625 \mathrm{E}-05$ & $1.41 \mathrm{E}-05$ & & & & & \\
\hline $\mathrm{Ca}$ & 2 & $7.58521 \mathrm{E}-06$ & 1.79E-05 & $2.24 \mathrm{E}-05$ & 0.000102 & $4.93 \mathrm{E}-05$ & $4.9 \mathrm{E}-05$ & $2.54 \mathrm{E}-05$ \\
\hline $\mathrm{Cd}$ & 2 & $3.08691 \mathrm{E}-06$ & $3.97 \mathrm{E}-06$ & & & & $9.81 \mathrm{E}-07$ & $3.65 \mathrm{E}-06$ \\
\hline $\mathrm{Co}$ & 2 & $1.54582 \mathrm{E}-05$ & $1.83 \mathrm{E}-05$ & & & $8.85 \mathrm{E}-06$ & $8.28 \mathrm{E}-06$ & \\
\hline $\mathrm{Cr}$ (CrO4) & -2 & 0.0002981 & 0.000298 & $2.11 \mathrm{E}-05$ & & $6.69 \mathrm{E}-06$ & $8.24 \mathrm{E}-06$ & \\
\hline $\mathrm{Cu}$ & 2 & $1.0575 \mathrm{E}-05$ & $1.13 \mathrm{E}-05$ & & & $8.08 \mathrm{E}-06$ & $9.81 \mathrm{E}-06$ & \\
\hline $\mathrm{Fe}$ & 3 & $8.93513 \mathrm{E}-06$ & $1.01 \mathrm{E}-05$ & & & $2.77 \mathrm{E}-05$ & $2.7 \mathrm{E}-05$ & \\
\hline $\mathrm{K}(\mathrm{AA})$ & 1 & 0.001992429 & 0.002041 & 0.001465 & 0.00139 & 0.0002 & 0.000188 & 0 \\
\hline $\mathrm{La}$ & 3 & $1.15182 \mathrm{E}-05$ & $1.22 \mathrm{E}-05$ & $2.44 \mathrm{E}-05$ & & $4.02 \mathrm{E}-05$ & $4.67 \mathrm{E}-05$ & \\
\hline $\mathrm{Li}$ & 1 & $6.09422 \mathrm{E}-05$ & 0.0002 & & & $8.86 \mathrm{E}-05$ & $9.53 \mathrm{E}-05$ & \\
\hline $\mathrm{Mg}$ & 2 & & & & $5.06 \mathrm{E}-06$ & 3.67E-06 & $3.84 \mathrm{E}-06$ & \\
\hline $\mathrm{Mn}$ & 2 & $2.56653 \mathrm{E}-06$ & $2.97 \mathrm{E}-06$ & & & & & \\
\hline Mo (MoO4) & -2 & $1.78236 \mathrm{E}-05$ & $1.66 \mathrm{E}-05$ & & & & & $5.31 \mathrm{E}-06$ \\
\hline $\mathrm{Na}$ & 1 & 0.07881837 & 0.078947 & 0.082298 & 0.081075 & 0.031433 & 0.035178 & 0.045199 \\
\hline $\mathrm{Ni}$ & 2 & $1.95945 \mathrm{E}-05$ & $1.61 \mathrm{E}-05$ & & $1.59 \mathrm{E}-05$ & $3.33 \mathrm{E}-05$ & $3.69 \mathrm{E}-05$ & \\
\hline \begin{tabular}{|l}
$\mathrm{P}(\mathrm{PO} 4)$ \\
\end{tabular} & -3 & $1.15182 \mathrm{E}-05$ & $1.22 \mathrm{E}-05$ & & & & & $4.88 \mathrm{E}-06$ \\
\hline $\mathrm{Pb}$ & 2 & $2.17664 \mathrm{E}-05$ & $2.3 \mathrm{E}-05$ & & $2.46 \mathrm{E}-05$ & $1.2 \mathrm{E}-05$ & & \\
\hline $\mathrm{Si}(\mathrm{HSiO} 4)$ & -1 & 0.000242114 & 0.00022 & 0.000972 & 0.000732 & 0.000833 & 0.000887 & 0.000171 \\
\hline
\end{tabular}


WSRC-TR-2002-00319 Rev. 0

SRT-RPP-2002-00171

\begin{tabular}{|c|c|c|c|c|c|c|c|c|}
\hline ICP-AES & charge & AZ-102 Sample 1 & AZ-102 Sample 2 & AN-105 Sample 1 & AN-105 Sample 2 & \begin{tabular}{|c|} 
AN-107 Sample \\
1 \\
\end{tabular} & AN-107 Sample 2 & AN-103 Sample 1 \\
\hline Sn & 2 & & & & & $2.38 \mathrm{E}-05$ & $3.19 \mathrm{E}-05$ & \\
\hline $\mathrm{Ti}$ & 3 & $3.92605 \mathrm{E}-06$ & $4.03 \mathrm{E}-06$ & & & & & \\
\hline $\mathrm{U}$ & 4 & $2.71397 \mathrm{E}-07$ & $3.21 \mathrm{E}-07$ & & & & & \\
\hline $\mathrm{V}$ & 3 & 4.3692E-06 & $4.62 \mathrm{E}-06$ & & & $3.44 \mathrm{E}-06$ & $3.78 \mathrm{E}-06$ & \\
\hline $\mathrm{Zn}$ & 2 & & & & & $3.77 \mathrm{E}-06$ & $3.53 \mathrm{E}-06$ & \\
\hline $\mathrm{Zr}$ & 4 & 4.29729E-05 & 4.22E-05 & & & & & \\
\hline \multicolumn{9}{|l|}{ IC } \\
\hline $\mathrm{Cl}$ & -1 & 0.009646574 & 0.009759 & 0.000628 & 0.000698 & 0.004265 & 0.006445 & \\
\hline $\mathrm{NO} 2$ & -1 & 0.012563579 & 0.012629 & 0.002302 & 0.002447 & 0.009243 & 0.009511 & 0.002954 \\
\hline $\mathrm{NO} 3$ & -1 & 0.011208773 & 0.011225 & 0.004654 & 0.005102 & 0.006858 & 0.007057 & 0.026303 \\
\hline $\mathrm{C} 2 \mathrm{O} 4$ & -2 & 0.00070893 & 0.000709 & & & & & \\
\hline SO4 & -2 & 0.006214607 & 0.006204 & & & & & \\
\hline $\mathrm{OH}$ & -1 & 0.002941176 & 0.002941 & 0.002929 & 0.002929 & 0.002828 & 0.002828 & 0.002955 \\
\hline TIC (CO3) & -2 & 0.015333333 & 0.015417 & 0.036744 & 0.035957 & 0.004331 & 0.004861 & 0.006224 \\
\hline \multicolumn{9}{|l|}{ GEA } \\
\hline \begin{tabular}{|l|} 
Sr-90 \\
\end{tabular} & 2 & & & & & & & $8.06 \mathrm{E}-10$ \\
\hline \multicolumn{9}{|l|}{ Cs-137 } \\
\hline \multicolumn{9}{|l|}{ ICP-MS } \\
\hline Tc-99(TcO4) & -1 & 0.00039596 & $4.01 \mathrm{E}-04$ & 0.000148 & 0.000148 & $3.15 \mathrm{E}-06$ & $3.17 \mathrm{E}-06$ & $1.18 \mathrm{E}-04$ \\
\hline
\end{tabular}

Prior to the charge balance, the amounts of $\mathrm{Na}^{+}$and $\mathrm{K}^{+}$combined made up at least 98.9 mole $\%$ of the total cations present in all samples. $\mathrm{Na}^{+}$was never less than 90 mole\%, and as high as 99 mole\%. Therefore, $\mathrm{Na}^{+}$and $\mathrm{K}^{+}$were considered to be the only significant cations. Also, because the concentration of $\mathrm{Na}^{+}$was consistently high, the effect on the physical properties due to variations in the $\mathrm{Na}^{+}$to $\mathrm{K}^{+}$ratio (within these concentration ranges) was thought to be negligible, therefore, $\mathrm{Na}^{+}$and $\mathrm{K}^{+}$were not included as variables in the mathematical models. Sodium and potassium were fixed at $97.5 \mathrm{~mole}^{+}{ }^{+}$and 2.5 mole $\% \mathrm{~K}^{+}$for all computer simulations.

The anions determined to be significant (based in large part on their relative concentrations) were $\mathrm{AlO}_{2}{ }^{-}$, $\mathrm{C}_{2} \mathrm{O}_{4}{ }^{-2}, \mathrm{CO}_{3}^{-2}, \mathrm{NO}_{2}^{-}, \mathrm{NO}_{3}{ }^{-}, \mathrm{OH}^{-}, \mathrm{SO}_{4}^{-2}$, and $\mathrm{TcO}_{4}^{-}$. No single anion was consistently dominant across all samples. $\mathrm{F}$ was below the minimum detection limits for all the reports, and $\mathrm{Cl}^{-}$was either below the detection limits, or small relative to the other anions.

The mathematical models are expressed in terms of the mass fraction of an anion relative to the total mass of the eight significant anions. They were expressed in terms of a dry weight basis because the endpoint of the eluate evaporation is a function only of the relative composition of the solids, and not effected by the relative amount of water initially present. (Regardless of the amount of water present in some fixed composition of dry solids, the endpoint is identical, only the amount of water removed changes making concentrations expressed in terms of water, such as molarity, inapplicable.)

The lower bound of a concentration range for an anion was defined by the minimum, non-zero, mass fraction of all eight eluate samples, The upper bound was determined by the maximum mass fraction of all eight eluate samples. In order for the entire concentration ranges of the anions to be simulated, a constraint must be imposed such that any anion mass fraction maximum summed with the remaining minimums be less than or equal to one. As all sums were less than one, there was no need for further modification of the concentration ranges. The resulting anion mass fraction ranges along with the molecular weights used for each are given in Table 3-1.

\subsection{Design of Experiment}

A statistical design of experiments was done [2] based on the concentration ranges of the significant species, resulting in a matrix of 129 design points (each representing an eluate composition vector), and is included in Appendix D. The physical properties of the concentrated eluate calculated by the computer simulations were fit to mathematical models of four different functional forms (polynomials of $\mathrm{P}^{\mathrm{st}}$ and/or $2^{\text {nd }}$ order in composition, with and without temperature cross terms). Although only four forms of the 
mathematical models were used, there are actually six trial expressions for each physical property because two of the forms were fit from two different sets of design points.

The first set contained sixteen design points and was used to fit two functional forms, equations (17) and (18) below. Both are linear in composition, with the addition of temperature cross terms in equation (18) (note that a maximum of 16 data points can be used to fit equation (18) without over specifying the equation). The second set consisted of 88 design points, including the 16 points of the first set, and was used to fit all four functional forms, equations $17-20$, as shown below. Equations (19) and (20) are both second order in composition, with temperature cross terms included in equation (20). The $\beta_{i}$ 's are the unknown coefficients to be determined by linear regression, Temp is the endpoint temperature in ${ }^{\circ} \mathrm{C}$, and the name of anion (e.g. [AlO2]) represents the mass fraction of that anion relative to the total mass of the eight significant anions.

$$
\begin{aligned}
\text { Response }_{\text {OLIESP }}= & \beta_{1}\left[\mathrm{AlO}_{2}\right]+\beta_{2}\left[\mathrm{C}_{2} \mathrm{O}_{4}\right]+\beta_{4}\left[\mathrm{CO}_{3}\right]+\beta_{7}\left[\mathrm{NO}_{2}\right]+\beta_{11}\left[\mathrm{NO}_{3}\right] \\
& +\beta_{16}[\mathrm{OH}]+\beta_{22}\left[\mathrm{SO}_{4}\right]+\beta_{29}\left[\mathrm{TcO}_{4}\right] \\
\text { Response }_{\text {OLI/ESP }}= & \beta_{1}\left[\mathrm{AlO}_{2}\right]+\beta_{2}\left[\mathrm{C}_{2} \mathrm{O}_{4}\right]+\beta_{4}\left[\mathrm{CO}_{3}\right]+\beta_{7}\left[\mathrm{NO}_{2}\right]+\beta_{11}\left[\mathrm{NO}_{3}\right] \\
& +\beta_{16}[\mathrm{OH}]+\beta_{22}\left[\mathrm{SO}_{4}\right]+\beta_{29}\left[\mathrm{TcO}_{4}\right]+\beta_{37}\left[\mathrm{AlO}_{2}\right] \mathrm{Temp} \\
& +\beta_{38}\left[\mathrm{C}_{2} \mathrm{O}_{4}\right] \text { Temp }+\beta_{40}\left[\mathrm{CO}_{3}\right] \text { Temp }+\beta_{43}\left[\mathrm{NO}_{2}\right] \text { Temp } \\
& +\beta_{47}\left[\mathrm{NO}_{3}\right] \text { Temp }+\beta_{52}[\mathrm{OH}] \text { Temp }+\beta_{58}\left[\mathrm{SO}_{4}\right] \text { Temp }+\beta_{65}\left[\mathrm{TcO}_{4}\right] \text { Temp }
\end{aligned}
$$

$$
\begin{aligned}
& \text { Response }_{\text {OLIESP }}=\beta_{1}\left[\mathrm{AlO}_{2}\right]+\beta_{2}\left[\mathrm{C}_{2} \mathrm{O}_{4}\right]+\beta_{3}\left[\mathrm{AlO}_{2}\right]\left[\mathrm{C}_{2} \mathrm{O}_{4}\right]+\beta_{4}\left[\mathrm{CO}_{3}\right]+\beta_{\{}[\mathrm{AlO}]\left[\mathrm{CO}_{3}\right] \\
& +\beta_{6}\left[\mathrm{C}_{2} \mathrm{O}_{4}\right]\left[\mathrm{CO}_{3}\right]+\beta_{7}\left[N \mathrm{NO}_{2}\right]+\beta_{8}\left[\mathrm{AlO}_{2}\right]\left[\mathrm{NO}_{2}\right]+\beta_{9}\left[\mathrm{C}_{2} \mathrm{O}_{4}\right]\left[N \mathrm{NO}_{2}\right] \\
& +\beta_{10}\left[\mathrm{CO}_{3}\right]\left[N O_{2}\right]+\beta_{11}\left[N O_{3}\right]+\beta_{12}\left[\mathrm{AlO}_{2}\right]\left[N O_{3}\right]+\beta_{13}\left[\mathrm{C}_{2} \mathrm{O}_{4}\right]\left[N O_{3}\right] \\
& +\beta_{14}\left[\mathrm{CO}_{3}\right]\left[\mathrm{NO}_{3}\right]+\beta_{15}\left[\mathrm{NO}_{2}\right]\left[\mathrm{NO}_{3}\right]+\beta_{16}[\mathrm{OH}]+\beta_{17}\left[\mathrm{AlO}_{2}\right][\mathrm{OH}] \\
& +\beta_{18}\left[\mathrm{C}_{2} \mathrm{O}_{4}\right][\mathrm{OH}]+\beta_{19}\left[\mathrm{CO}_{3}\right][\mathrm{OH}]+\beta_{20}\left[\mathrm{NO}_{2}\right][\mathrm{OH}]+\beta_{21}\left[\mathrm{NO}_{3}\right][\mathrm{OH}] \\
& +\beta_{22}\left[\mathrm{SO}_{4}\right]+\beta_{23}\left[\mathrm{AlO}_{2}\right]\left[\mathrm{SO}_{4}\right]+\beta_{24}\left[\mathrm{C}_{2} \mathrm{O}_{4}\right]\left[\mathrm{SO}_{4}\right]+\beta_{25}\left[\mathrm{CO}_{3}\right]\left[\mathrm{SO}_{4}\right] \\
& +\beta_{26}\left[\mathrm{NO}_{2}\right]\left[\mathrm{SO}_{4}\right]+\beta_{27}\left[\mathrm{NO}_{3}\right]\left[\mathrm{SO}_{4}\right]+\beta_{28}[\mathrm{OH}]\left[\mathrm{SO}_{4}\right] \\
& +\beta_{29}\left[\mathrm{TcO}_{4}\right]+\beta_{30}\left[\mathrm{AlO}_{2}\right]\left[\mathrm{TcO}_{4}\right]+\beta_{31}\left[\mathrm{C}_{2} \mathrm{O}_{4}\right]\left[\mathrm{TcO}_{4}\right] \\
& +\beta_{32}\left[\mathrm{CO}_{3}\right]\left[\mathrm{TcO}_{4}\right]+\beta_{32}\left[\mathrm{NO}_{2}\right]\left[\mathrm{TcO}_{4}\right]+\beta_{34}\left[\mathrm{NO}_{3}\right]\left[\mathrm{TcO}_{4}\right]_{4} \\
& +\beta_{35}[\mathrm{OH}]\left[\mathrm{TcO}_{4}\right]+\beta_{36}\left[\mathrm{SO}_{4}\right]\left[\mathrm{TcO}_{4}\right]
\end{aligned}
$$




$$
\begin{aligned}
& \text { Response }_{\text {OLIESP }}=\beta_{1}\left[\mathrm{AlO}_{2}\right]+\beta_{2}\left[\mathrm{C}_{2} \mathrm{O}_{4}\right]+\beta_{3}\left[\mathrm{AlO}_{2}\right]\left[\mathrm{C}_{2} \mathrm{O}_{4}\right]+\beta_{4}\left[\mathrm{CO}_{3}\right]+\beta_{5}\left[\mathrm{AlO}_{2}\right]\left[\mathrm{CO}_{3}\right] \\
& +\beta_{6}\left[C_{2} O_{4}\right]\left[C O_{3}\right]+\beta_{7}\left[N O_{2}\right]+\beta_{5}\left[A l O_{2}\right]\left[N O_{2}\right]+\beta_{9}\left[C_{2} O_{4}\right]\left[N O_{2}\right] \\
& +\beta_{10}\left[\mathrm{CO}_{3}\right]\left[N \mathrm{NO}_{2}\right]+\beta_{11}\left[N O_{3}\right]+\beta_{12}\left[\mathrm{AlO}_{2}\right]\left[N O_{3}\right]+\beta_{13}\left[\mathrm{C}_{2} \mathrm{O}_{4}\right]\left[N O_{3}\right] \\
& +\beta_{14}\left[\mathrm{CO}_{3}\right]\left[\mathrm{NO}_{3}\right]+\beta_{15}\left[\mathrm{NO}_{2}\right]\left[\mathrm{NO}_{3}\right]+\beta_{16}[\mathrm{OH}]+\beta_{17}\left[\mathrm{AlO}_{2}\right][\mathrm{OH}] \\
& +\beta_{18}\left[\mathrm{C}_{2} \mathrm{O}_{4}\right][\mathrm{OH}]+\beta_{19}\left[\mathrm{CO}_{3}\right][\mathrm{OH}]+\beta_{2 d}\left[\mathrm{NO}_{2}\right][\mathrm{OH}]+\beta_{2[}\left[\mathrm{NO}_{3}\right][\mathrm{OH}] \\
& +\beta_{22}\left[\mathrm{SO}_{4}\right]+\beta_{23}\left[\mathrm{AlO}_{2}\right]\left[\mathrm{SO}_{4}\right]+\beta_{24}\left[\mathrm{C}_{2} \mathrm{O}_{4}\right]\left[\mathrm{SO}_{4}\right]+\beta_{25}\left[\mathrm{CO}_{3}\right]\left[\mathrm{SO}_{4}\right] \\
& +\beta_{26}\left[\mathrm{NO}_{2}\right]\left[\mathrm{SO}_{4}\right]+\beta_{27}\left[\mathrm{NO}_{3}\right]\left[\mathrm{SO}_{4}\right]+\beta_{28}[\mathrm{OH}]\left[\mathrm{SO}_{4}\right] \\
& +\beta_{29}\left[\mathrm{TcO}_{4}\right]+\beta_{30}\left[\mathrm{AlO}_{2}\right]\left[\mathrm{TcO}_{4}\right]+\beta_{31}\left[\mathrm{C}_{2} \mathrm{O}_{4}\right]\left[\mathrm{TcO}_{4}\right] \\
& +\beta_{32}\left[\mathrm{CO}_{3}\right]\left[\mathrm{TcO}_{4}\right]+\beta_{33}\left[\mathrm{NO}_{2}\right]\left[\mathrm{TcO} \mathrm{O}_{4}\right]+\beta_{34}\left[\mathrm{NO}_{3}\right]\left[\mathrm{TcO}_{4}\right]_{4} \\
& +\beta_{35}[\mathrm{OH}]\left[\mathrm{TcO}_{4}\right]+\beta_{36}\left[\mathrm{SO}_{4}\right]\left[\mathrm{TcO}_{4}\right]+\beta_{37}\left[\mathrm{AlO}_{2}\right] \mathrm{Temp}+\beta_{38}\left[\mathrm{C}_{2} \mathrm{O}_{4}\right] \mathrm{Temp} \\
& +\beta_{39}\left[\mathrm{AlO}_{2}\right]\left[\mathrm{C}_{2} \mathrm{O}_{4}\right] \mathrm{Temp}+\beta_{40}\left[\mathrm{CO}_{3}\right] \mathrm{Temp}+\beta_{41}\left[\mathrm{AlO}_{2}\right]\left[\mathrm{CO}_{3}\right] \mathrm{Temp} \\
& +\beta_{42}\left[\mathrm{C}_{2} \mathrm{O}_{4}\right]\left[\mathrm{CO}_{3}\right] \text { Temp }+\beta_{43}\left[\mathrm{NO}_{2}\right] \text { Temp }+\beta_{44}\left[\mathrm{AlO}_{2}\right]\left[\mathrm{NO}_{2}\right] \text { Temp } \\
& +\beta_{45}\left[\mathrm{C}_{2} \mathrm{O}_{4}\right]\left[\mathrm{NO}_{2}\right] \mathrm{Temp}+\beta_{46}\left[\mathrm{CO}_{3}\right]\left[\mathrm{NO}_{2}\right] \text { Temp }+\beta_{47}\left[\mathrm{NO}_{3}\right] \text { Temp } \\
& +\beta_{48}\left[\mathrm{AlO}_{2}\right]\left[\mathrm{NO}_{3}\right] \text { Temp }+\beta_{49}\left[\mathrm{C}_{2} \mathrm{O}_{4}\right]\left[\mathrm{NO}_{3}\right] \mathrm{Temp}+\beta_{50}\left[\mathrm{CO}_{3}\right]\left[\mathrm{NO}_{3}\right] \text { Temp } \\
& +\beta_{51}\left[\mathrm{NO}_{2}\right]\left[\mathrm{NO}_{3}\right] \mathrm{Temp}+\beta_{52}[\mathrm{OH}] \mathrm{Temp}+\beta_{53}\left[\mathrm{AlO} \mathrm{O}_{2}\right][\mathrm{OH}] \mathrm{Temp} \\
& +\beta_{54}\left[\mathrm{C}_{2} \mathrm{O}_{4}\right][\mathrm{OH}] \mathrm{Temp}+\beta_{55}\left[\mathrm{CO}_{3}\right][\mathrm{OH}] \mathrm{Temp}+\beta_{56}\left[\mathrm{NO}_{2}\right][\mathrm{OH}] \text { Temp } \\
& +\beta_{57}\left[\mathrm{NO}_{3}\right][\mathrm{OH}] \mathrm{Temp}+\beta_{58}\left[\mathrm{SO}_{4}\right] \mathrm{Temp}+\beta_{59}\left[\mathrm{AlO}_{2}\right]\left[\mathrm{SO}_{4}\right] \text { Temp } \\
& +\beta_{60}\left[\mathrm{C}_{2} \mathrm{O}_{4}\right]\left[\mathrm{SO}_{4}\right] \mathrm{Temp}+\beta_{61}\left[\mathrm{CO}_{3}\right]\left[\mathrm{SO}_{4}\right] \mathrm{Temp}+\beta_{62}\left[\mathrm{NO}_{2}\right]\left[\mathrm{SO}_{4}\right] \mathrm{Temp} \\
& +\beta_{63}\left[\mathrm{NO}_{3}\right]\left[\mathrm{SO}_{4}\right] \mathrm{Temp}+\beta_{64}[\mathrm{OH}]\left[\mathrm{TcO}_{4}\right] \mathrm{Temp}+\beta_{65}\left[\mathrm{TcO} \mathrm{O}_{4}\right] \mathrm{Temp} \\
& +\beta_{66}\left[\mathrm{AlO}_{2}\right]\left[\mathrm{TcO}_{4}\right] \mathrm{Temp}+\beta_{67}\left[\mathrm{C}_{2} \mathrm{O}_{4}\right]\left[\mathrm{TcO}_{4}\right] \mathrm{Temp}+\beta_{68}\left[\mathrm{CO}_{3}\right]\left[\mathrm{TcO} \mathrm{O}_{4}\right] \mathrm{Temp}
\end{aligned}
$$

The remaining 41 design points were derived using the Orthogonal Latin Hypercube (OLH) method [3] designed to generate independent ("space filling") composition vectors evenly distributed across the concentration ranges. The mixture aspect of this problem prevented the use of the OLH method in its purest form. The solution composition had to satisfy a constraint. That is, they had to sum to one.

These OLH points were not used to fit the models. Instead, they were used as test points to compare the predictions of the trial mathematical models to the simulation results, providing a measure of the relative success of the trial models. The fitted model's inability to mirror the simulation results exactly reflects a bias in the model. The intent is to develop models that do not demonstrate a large bias over the OLH points. The design points which were simulated are included in Table 3-3 below. Two values are listed in the temperature column, the left values were used for the lower temperature range, and the right for the upper temperature range. The simulation results of points 1 though 16 were fit to equations (17) and (18). Points 17 through 57 (highlighted) are the OLH test points, and points 58 through 129 combined with the first 16 points are the 88 points used to fit to all four equations 17-20. Two values are listed in the temperature column, the left value was used in simulations for the lower temperature range, and the right for the upper temperature range. 
WSRC-TR-2002-00319 Rev. 0

SRT-RPP-2002-00171

Table 3-3. Design Point Compositions Simulated using OLI/ESP (highlighted rows are the OLH points)

\begin{tabular}{|c|c|c|c|c|c|c|c|c|c|}
\hline Run & \begin{tabular}{|l|}
$\mathrm{A} 1 \mathrm{O} 2$ \\
Mass fraction
\end{tabular} & \begin{tabular}{|l|}
$\mathrm{C} 2 \mathrm{O} 4$ \\
mass fraction \\
\end{tabular} & $\begin{array}{l}\mathrm{CO} 3 \\
\text { mass fraction }\end{array}$ & \begin{tabular}{|l|}
$\mathrm{NO} 2$ \\
mass fraction \\
\end{tabular} & $\begin{array}{l}\mathrm{NO3} \\
\text { mass fraction }\end{array}$ & \begin{tabular}{|l|}
$\mathrm{OH}$ \\
mass fraction
\end{tabular} & \begin{tabular}{|l|}
$\mathrm{SO} 4$ \\
mass fraction
\end{tabular} & \begin{tabular}{|l|}
$\mathrm{TcO} 4$ \\
mass fraction
\end{tabular} & \begin{tabular}{|l|} 
Temperature \\
$\left({ }^{\circ} \mathrm{C}\right)$ \\
\end{tabular} \\
\hline RTS001 & 0.0075 & 0.00001 & 0.805 & 0.039 & 0.10706 & 0.041 & 0.00001 & 0.00042 & 20 or 35.369 \\
\hline $\begin{array}{ll}\text { RTS002 } \\
\end{array}$ & 0.0075 & 0.0205 & 0.5955 & 0.039 & 0.105 & 0.016 & 0.195 & 0.0215 & 20 or 35.369 \\
\hline RTS003 & 0.03 & 0.0205 & 0.42199 & 0.36 & 0.105 & 0.041 & 0.00001 & 0.0215 & 20 or 35.369 \\
\hline RTS004 & 0.03 & 0.00001 & 0.29357 & 0.36 & 0.105 & 0.016 & 0.195 & 0.00042 & 20 or 35.369 \\
\hline $\begin{array}{ll}\text { RTS005 } \\
\end{array}$ & 0.0075 & 0.0205 & 0.165 & 0.36 & 0.43057 & 0.016 & 0.00001 & 0.00042 & 20 or 35.369 \\
\hline RTS006 & 0.0075 & 0.00001 & 0.165 & 0.36 & 0.20999 & 0.041 & 0.195 & 0.0215 & 20 or 35.369 \\
\hline RTS007 & 0.02348 & 0.00001 & 0.165 & 0.039 & 0.735 & 0.016 & 0.00001 & 0.0215 & 20 or 35.369 \\
\hline RTS008 & 0.03 & 0.0205 & 0.165 & 0.039 & 0.50908 & 0.041 & 0.195 & 0.00042 & 20 or 35.369 \\
\hline $\begin{array}{l}\text { RTS009 } \\
\end{array}$ & 0.0075 & 0.00001 & 0.805 & 0.039 & 0.10706 & 0.041 & 0.00001 & 0.00042 & 35.37 or 70 \\
\hline $\begin{array}{ll}\text { RTS010 } \\
\end{array}$ & 0.0075 & 0.0205 & 0.5955 & 0.039 & 0.105 & 0.016 & 0.195 & 0.0215 & 35.37 or 70 \\
\hline RTS011 & 0.03 & 0.0205 & 0.42199 & 0.36 & 0.105 & 0.041 & 0.00001 & 0.0215 & 35.37 or 70 \\
\hline $\begin{array}{ll}\text { RTS012 } \\
\end{array}$ & 0.03 & 0.00001 & 0.29357 & 0.36 & 0.105 & 0.016 & 0.195 & 0.00042 & 35.37 or 70 \\
\hline RTS013 & 0.0075 & 0.0205 & 0.165 & 0.36 & 0.43057 & 0.016 & 0.00001 & 0.00042 & 35.37 or 70 \\
\hline RTS014 & 0.0075 & 0.00001 & 0.165 & 0.36 & 0.20999 & 0.041 & 0.195 & 0.0215 & 35.37 or 70 \\
\hline $\begin{array}{ll}\text { RTS015 } \\
\end{array}$ & 0.02348 & 0.00001 & 0.165 & 0.039 & 0.735 & 0.016 & 0.00001 & 0.0215 & 35.37 or 70 \\
\hline RTS016 & 0.03 & 0.0205 & 0.165 & 0.039 & 0.50908 & 0.041 & 0.195 & 0.00042 & 35.37 or 70 \\
\hline RTS017 & 0.01945 & 0.00897 & 0.32527 & 0.15937 & 0.2625 & 0.016 & 0.18891 & 0.01952 & 32.01 or 62.42 \\
\hline \begin{tabular}{|l|l|} 
RTS018 \\
\end{tabular} & 0.02016 & 0.0109 & 0.49883 & 0.16941 & 0.28219 & 0.01678 & 0.00001 & 0.00174 & 22.88 or 41.86 \\
\hline RTS019 & 0.02086 & 0.00769 & 0.39332 & 0.21956 & 0.30188 & 0.01756 & 0.01829 & 0.02084 & 32.97 or 64.59 \\
\hline RTS020 & 0.02156 & 0.01218 & 0.23359 & 0.20953 & 0.32156 & 0.01834 & 0.18281 & 0.00042 & 21.92 or 39.70 \\
\hline RTS021 & 0.02297 & 0.01346 & 0.29339 & 0.12928 & 0.47906 & 0.01991 & 0.02438 & 0.01755 & 20.96 or 37.53 \\
\hline RTS022 & 0.02367 & 0.00513 & 0.18507 & 0.25969 & 0.45937 & 0.02069 & 0.04266 & 0.00371 & 34.89 or 68.92 \\
\hline RTS023 & 0.02508 & 0.00385 & 0.59778 & 0.07913 & 0.105 & 0.03475 & 0.14016 & 0.01425 & 27.20 or 51.60 \\
\hline RTS024 & 0.02578 & 0.01602 & 0.65462 & 0.08916 & 0.12469 & 0.03397 & 0.04876 & 0.00701 & 28.65 or 54.85 \\
\hline RTS025 & 0.02648 & 0.00257 & 0.41096 & 0.29981 & 0.14438 & 0.03319 & 0.06704 & 0.01557 & 26.24 or 49.44 \\
\hline RTS026 & 0.02719 & 0.0173 & 0.32951 & 0.28978 & 0.16406 & 0.03241 & 0.13407 & 0.00569 & 29.61 or 57.01 \\
\hline RTS027 & 0.01875 & 0.01026 & 0.21453 & 0.1995 & 0.42 & 0.0285 & 0.0975 & 0.01096 & 27.68 or 52.69 \\
\hline RTS028 & 0.01594 & 0.00833 & 0.19547 & 0.18947 & 0.51844 & 0.03866 & 0.0122 & 0.0215 & 33.45 or 65.67 \\
\hline RTS029 & 0.01523 & 0.0141 & 0.26443 & 0.27975 & 0.34125 & 0.03787 & 0.03048 & 0.01689 & 21.44 or 38.62 \\
\hline RTS030 & 0.01383 & 0.01538 & 0.24399 & 0.13931 & 0.38062 & 0.03631 & 0.15235 & 0.01821 & 20.48 or 36.45 \\
\hline RTS031 & 0.01313 & 0.00577 & 0.35629 & 0.14934 & 0.40031 & 0.03553 & 0.03657 & 0.00306 & 35.37 or 70.00 \\
\hline RTS032 & 0.00961 & 0.01922 & 0.3112 & 0.36 & 0.18375 & 0.02538 & 0.07922 & 0.01162 & 30.09 or 58.10 \\
\hline RTS033 & 0.00891 & 0.00193 & 0.27808 & 0.34997 & 0.20344 & 0.02616 & 0.12188 & 0.00964 & 24.80 or 46.19 \\
\hline RTS034 & 0.0082 & 0.0205 & 0.54564 & 0.05906 & 0.22312 & 0.02694 & 0.1036 & 0.01294 & 31.05 or 60.26 \\
\hline RTS035 & 0.0075 & 0.00065 & 0.55858 & 0.06909 & 0.24281 & 0.02772 & 0.08532 & 0.00833 & 23.84 or 44.03 \\
\hline RTS036 & 0.01917 & 0.00949 & 0.28098 & 0.17543 & 0.3255 & 0.021 & 0.15234 & 0.01609 & 32.01 or 62.42 \\
\hline RTS037 & 0.01959 & 0.01064 & 0.38511 & 0.18144 & 0.33731 & 0.02147 & 0.03901 & 0.00542 & 22.88 or 41.86 \\
\hline RTS038 & 0.02002 & 0.00872 & 0.3218 & 0.21154 & 0.34913 & 0.02194 & 0.04998 & 0.01688 & 32.97 or 64.59 \\
\hline RTS039 & 0.02044 & 0.01141 & 0.22597 & 0.20552 & 0.36094 & 0.02241 & 0.14869 & 0.00463 & 21.92 or 39.70 \\
\hline $\begin{array}{ll}\text { RTS040 } \\
\end{array}$ & 0.02086 & 0.00795 & 0.1846 & 0.15135 & 0.46725 & 0.02288 & 0.13772 & 0.0074 & 33.93 or 66.75 \\
\hline \begin{tabular}{|l|} 
RTS041 \\
\end{tabular} & 0.02128 & 0.01218 & 0.26185 & 0.15737 & 0.45544 & 0.02334 & 0.05363 & 0.01491 & 20.96 or 37.53 \\
\hline RTS042 & 0.0217 & 0.00718 & 0.19686 & 0.23561 & 0.44362 & 0.02381 & 0.0646 & 0.00661 & 34.89 or 68.92 \\
\hline RTS042 & 0.02255 & 0.00641 & 0.44449 & 0.12728 & 0.231 & 0.03225 & 0.1231 & 0.01293 & $27.20 \backslash$ or 51.60 \\
\hline RTS044 & 0.02297 & 0.01371 & 0.47859 & 0.13329 & 0.24281 & 0.03178 & 0.06826 & 0.00858 & 28.65 or 54.85 \\
\hline RTS045 & 0.02339 & 0.00565 & 0.33239 & 0.25969 & 0.25462 & 0.03131 & 0.07923 & 0.01372 & 26.24 or 49.44 \\
\hline \begin{tabular}{|l|l|l} 
RTS046 & \\
\end{tabular} & 0.02381 & 0.01448 & 0.28352 & 0.25367 & 0.26644 & 0.03084 & 0.11944 & 0.00779 & 29.61 or $\backslash 57.01$ \\
\hline RTS047 & 0.02466 & 0.01525 & 0.17641 & 0.10922 & 0.54994 & 0.02991 & 0.08288 & 0.01175 & 30.57 or 59.18 \\
\hline RTS048 & 0.01875 & 0.01026 & 0.21454 & 0.1995 & 0.42 & 0.0285 & 0.0975 & 0.01095 & 27.68 or 52.69 \\
\hline RTS049 & 0.01706 & 0.0091 & 0.2031 & 0.19348 & 0.47906 & 0.03459 & 0.04632 & 0.01728 & 33.45 or 65.67 \\
\hline RTS050 & 0.01664 & 0.01256 & 0.24447 & 0.24765 & 0.37275 & 0.03413 & 0.05729 & 0.01451 & 21.44 or 38.62 \\
\hline RTS051 & 0.01622 & 0.00833 & 0.16722 & 0.24163 & 0.38456 & 0.03366 & 0.14138 & 0.007 & 34.41 or 67.84 \\
\hline RTS052 & 0.0158 & 0.01333 & 0.23221 & 0.16339 & 0.39637 & 0.03319 & 0.13041 & 0.0153 & 20.48 or 36.45 \\
\hline $\begin{array}{ll}\text { RTS053 } \\
\end{array}$ & 0.01538 & 0.00757 & 0.29959 & 0.16941 & 0.40819 & 0.03272 & 0.06095 & 0.00621 & 35.37 or 70.00 \\
\hline RTS054 & 0.01327 & 0.01563 & 0.27254 & 0.2958 & 0.27825 & 0.02662 & 0.08654 & 0.01135 & .10 \\
\hline RTS055 & 0.01284 & 0.00526 & 0.25266 & 0.28978 & 0.29006 & 0.02709 & 0.11213 & 0.01016 & 24.80 \\
\hline
\end{tabular}


WSRC-TR-2002-00319 Rev. 0

SRT-RPP-2002-00171

\begin{tabular}{|c|c|c|c|c|c|c|c|c|c|}
\hline Run & $\begin{array}{l}\mathrm{A} 1 \mathrm{O} 2 \\
\text { Mass fraction }\end{array}$ & \begin{tabular}{|l|}
$\mathrm{C} 2 \mathrm{O} 4$ \\
mass fraction
\end{tabular} & \begin{tabular}{|l|}
$\mathrm{CO} 3$ \\
mass fraction
\end{tabular} & \begin{tabular}{|l|}
$\mathrm{NO} 2$ \\
mass fraction
\end{tabular} & $\begin{array}{l}\mathrm{NO} 3 \\
\text { mass fraction }\end{array}$ & \begin{tabular}{|l|}
$\mathrm{OH}$ \\
mass fraction
\end{tabular} & $\begin{array}{l}\mathrm{SO} 4 \\
\text { mass fraction }\end{array}$ & \begin{tabular}{|l|}
$\mathrm{TcO} 4$ \\
mass fraction
\end{tabular} & $\begin{array}{l}\text { Temperature } \\
\left({ }^{\circ} \mathrm{C}\right)\end{array}$ \\
\hline RTS056 & 0.01242 & 0.0164 & 0.4132 & 0.11524 & 0.30188 & 0.02756 & 0.10116 & 0.01214 & 31.05 or 60.26 \\
\hline RTS057 & 0.012 & 0.00449 & 0.42096 & 0.12126 & 0.31369 & 0.02803 & 0.09019 & 0.00937 & 23.84 or 44.03 \\
\hline RTS058 & 0.03 & 0.0205 & 0.38684 & 0.039 & 0.48223 & 0.041 & 0.00001 & 0.00042 & 20 or 35.369 \\
\hline \begin{tabular}{|l|} 
RTS059 \\
\end{tabular} & 0.01723 & 0.01528 & 0.61598 & 0.07408 & 0.23398 & 0.01898 & 0.00299 & 0.02149 & 20 or 35.369 \\
\hline $\begin{array}{l}\text { RTS060 } \\
\end{array}$ & 0.02999 & 0.02002 & 0.56696 & 0.12116 & 0.18596 & 0.03646 & 0.01797 & 0.02149 & 20 or 35.369 \\
\hline RTS061 & 0.02999 & 0.02049 & 0.45366 & 0.20006 & 0.20066 & 0.04099 & 0.03267 & 0.02149 & 20 or 35.369 \\
\hline RTS062 & 0.03 & 0.00001 & 0.49032 & 0.23313 & 0.15784 & 0.016 & 0.07228 & 0.00042 & 20 or 35.369 \\
\hline RTS063 & 0.02999 & 0.0205 & 0.74671 & 0.07367 & 0.10757 & 0.016 & 0.00514 & 0.00042 & 20 or 35.369 \\
\hline RTS064 & 0.0075 & 0.0205 & 0.30318 & 0.36 & 0.11269 & 0.041 & 0.15471 & 0.00042 & 20 or 35.369 \\
\hline RTS065 & 0.02999 & 0.02049 & 0.39088 & 0.13688 & 0.20288 & 0.04099 & 0.15639 & 0.02149 & 20 or 35.369 \\
\hline RTS066 & 0.0075 & 0.0205 & 0.63125 & 0.17445 & 0.11277 & 0.02401 & 0.0291 & 0.00042 & 20 or 35.369 \\
\hline RTS067 & 0.02999 & 0.02049 & 0.35878 & 0.23318 & 0.17078 & 0.04099 & 0.12429 & 0.02149 & 20 or 35.369 \\
\hline RTS068 & 0.0075 & 0.00001 & 0.57119 & 0.10488 & 0.105 & 0.016 & 0.195 & 0.00042 & 20 or 35.369 \\
\hline RTS069 & 0.0075 & 0.0205 & 0.78657 & 0.039 & 0.105 & 0.041 & 0.00001 & 0.00042 & 20 or 35.369 \\
\hline $\begin{array}{ll}\text { RTS070 } \\
\end{array}$ & 0.0075 & 0.0205 & 0.47262 & 0.18131 & 0.25382 & 0.041 & 0.00174 & 0.0215 & 20 or 35.369 \\
\hline RTS071 & 0.02999 & 0.02049 & 0.28648 & 0.12858 & 0.41448 & 0.04099 & 0.05749 & 0.02149 & 20 or 35.369 \\
\hline RTS072 & 0.01998 & 0.00002 & 0.73548 & 0.06558 & 0.16248 & 0.01601 & 0.00002 & 0.00043 & 20 or 35.369 \\
\hline $\begin{array}{ll}\text { RTS073 } \\
\end{array}$ & 0.02999 & 0.02049 & 0.27771 & 0.21591 & 0.28071 & 0.04099 & 0.11272 & 0.02149 & 20 or 35.369 \\
\hline RTS074 & 0.03 & 0.0205 & 0.5103 & 0.10959 & 0.30039 & 0.016 & 0.01279 & 0.00042 & 20 or 35.369 \\
\hline RTS075 & 0.03 & 0.00001 & 0.54132 & 0.1449 & 0.20807 & 0.041 & 0.0132 & 0.0215 & 20 or 35.369 \\
\hline RTS076 & 0.02999 & 0.02049 & 0.29533 & 0.16953 & 0.29733 & 0.04099 & 0.14591 & 0.00042 & 20 or 35.369 \\
\hline RTS077 & 0.02999 & 0.02049 & 0.52543 & 0.17573 & 0.14543 & 0.04099 & 0.06151 & 0.00042 & 20 or 35.369 \\
\hline RTS078 & 0.02999 & 0.00996 & 0.62295 & 0.14525 & 0.11495 & 0.02595 & 0.02946 & 0.02149 & 20 or 35.369 \\
\hline \begin{tabular}{|l|} 
RTS079 \\
\end{tabular} & 0.02991 & 0.02049 & 0.56916 & 0.15546 & 0.12516 & 0.03866 & 0.03967 & 0.02149 & 20 or 35.369 \\
\hline RTS080 & 0.0075 & 0.00001 & 0.39102 & 0.039 & 0.42542 & 0.016 & 0.12063 & 0.00042 & 20 or 35.369 \\
\hline RTS081 & 0.02999 & 0.02049 & 0.33216 & 0.20656 & 0.27016 & 0.04099 & 0.07817 & 0.02149 & 20 or 35.369 \\
\hline RTS082 & 0.02999 & 0.02049 & 0.34018 & 0.18248 & 0.27818 & 0.04099 & 0.08619 & 0.02149 & 20 or 35.369 \\
\hline $\begin{array}{ll}\text { RTS083 } \\
\end{array}$ & 0.03 & 0.00001 & 0.76873 & 0.039 & 0.12083 & 0.041 & 0.00001 & 0.00042 & 20 or 35.369 \\
\hline RTS084 & 0.02999 & 0.01495 & 0.68989 & 0.08399 & 0.11789 & 0.02889 & 0.0129 & 0.02149 & 20 or 35.369 \\
\hline RTS085 & 0.02999 & 0.02049 & 0.56736 & 0.12156 & 0.18636 & 0.03436 & 0.01837 & 0.02149 & 20 or 35.369 \\
\hline RTS086 & 0.03 & 0.02048 & 0.34753 & 0.13666 & 0.39046 & 0.01991 & 0.03347 & 0.0215 & 20 or 35.369 \\
\hline RTS087 & 0.0075 & 0.00001 & 0.66902 & 0.09266 & 0.1933 & 0.016 & 0.00001 & 0.0215 & 20 or 35.369 \\
\hline RTS088 & 0.02482 & 0.02049 & 0.68982 & 0.08392 & 0.11782 & 0.02882 & 0.01283 & 0.02149 & 20 or 35.369 \\
\hline RTS089 & 0.02999 & 0.01985 & 0.68249 & 0.10869 & 0.11049 & 0.02149 & 0.0055 & 0.02149 & 20 or 35.369 \\
\hline RTS090 & 0.0075 & 0.00001 & 0.31872 & 0.16098 & 0.44568 & 0.04099 & 0.02569 & 0.00042 & 20 or 35.369 \\
\hline RTS091 & 0.01116 & 0.01596 & 0.74466 & 0.07476 & 0.10866 & 0.01966 & 0.00367 & 0.02149 & 20 or 35.369 \\
\hline $\begin{array}{ll}\text { RTS092 } \\
\end{array}$ & 0.03 & 0.0205 & 0.41096 & 0.21296 & 0.25126 & 0.041 & 0.03291 & 0.00042 & 20 or 35.369 \\
\hline $\begin{array}{ll}\text { RTS093 } \\
\end{array}$ & 0.02999 & 0.02049 & 0.48576 & 0.10376 & 0.23276 & 0.04099 & 0.06477 & 0.02149 & 20 or 35.369 \\
\hline RTS094 & 0.03 & 0.0205 & 0.38684 & 0.039 & 0.48223 & 0.041 & 0.00001 & 0.00042 & 35.37 or 70 \\
\hline RTS095 & 0.01723 & 0.01528 & 0.61598 & 0.07408 & 0.23398 & 0.01898 & 0.00299 & 0.02149 & 35.37 or 70 \\
\hline RTS096 & 0.02999 & 0.02002 & 0.56696 & 0.12116 & 0.18596 & 0.03646 & 0.01797 & 0.02149 & 35.37 or 70 \\
\hline $\begin{array}{ll}\text { RTS097 } \\
\end{array}$ & 0.02999 & 0.02049 & 0.45366 & 0.20006 & 0.20066 & 0.04099 & 0.03267 & 0.02149 & 35.37 or 70 \\
\hline RTS098 & 0.03 & 0.00001 & 0.49032 & 0.23313 & 0.15784 & 0.016 & 0.07228 & 0.00042 & 35.37 or 70 \\
\hline RTS099 & 0.02999 & 0.0205 & 0.74671 & 0.07367 & 0.10757 & 0.016 & 0.00514 & 0.00042 & 35.37 or 70 \\
\hline RTS100 & 0.0075 & 0.0205 & 0.30318 & 0.36 & 0.11269 & 0.041 & 0.15471 & 0.00042 & 35.37 or 70 \\
\hline RTS101 & 0.02999 & 0.02049 & 0.39088 & 0.13688 & 0.20288 & 0.04099 & 0.15639 & 0.02149 & 35.37 or 70 \\
\hline RTS102 & 0.0075 & 0.0205 & 0.63125 & 0.17445 & 0.11277 & 0.02401 & 0.0291 & 0.00042 & 35.37 or 70 \\
\hline RTS103 & 0.02999 & 0.02049 & 0.35878 & 0.23318 & 0.17078 & 0.04099 & 0.12429 & 0.02149 & 35.37 or 70 \\
\hline RTS104 & 0.0075 & 0.00001 & 0.57119 & 0.10488 & 0.105 & 0.016 & 0.195 & 0.00042 & 35.37 or 70 \\
\hline RTS105 & 0.0075 & 0.0205 & 0.78657 & 0.039 & 0.105 & 0.041 & 0.00001 & 0.00042 & 35.37 or 70 \\
\hline RTS106 & 0.0075 & 0.0205 & 0.47262 & 0.18131 & 0.25382 & 0.041 & 0.00174 & 0.0215 & 35.37 or 70 \\
\hline RTS107 & 0.02999 & 0.02049 & 0.28648 & 0.12858 & 0.41448 & 0.04099 & 0.05749 & 0.02149 & 35.37 or 70 \\
\hline RTS108 & 0.01998 & 0.00002 & 0.73548 & 0.06558 & 0.16248 & 0.01601 & 0.00002 & 0.00043 & 35.37 or 70 \\
\hline RTS109 & 0.02999 & 0.02049 & 0.27771 & 0.21591 & 0.28071 & 0.04099 & 0.11272 & 0.02149 & 35.37 or 70 \\
\hline RTS110 & 0.03 & 0.0205 & 0.5103 & 0.10959 & 0.30039 & 0.016 & 0.01279 & 0.00042 & 35.37 or 70 \\
\hline RTS111 & 0.03 & 0.00001 & 0.54132 & 0.1449 & 0.20807 & 0.041 & 0.0132 & 0.0215 & 35.37 or 70 \\
\hline RTS112 & 0.02999 & 0.02049 & 0.29533 & 0.16953 & 0.29733 & 0.04099 & 0.14591 & 0.00042 & 35.37 or 70 \\
\hline RTS113 & 0.02999 & 0.02049 & 0.52543 & 0.17573 & 0.14543 & 0.04099 & 0.06151 & 0.00042 & 35.37 or 70 \\
\hline
\end{tabular}


WSRC-TR-2002-00319 Rev. 0

SRT-RPP-2002-00171

\begin{tabular}{|l|c|c|c|c|c|c|c|c|c|}
\hline Run & $\begin{array}{l}\text { A1O2 } \\
\text { Mass fraction }\end{array}$ & $\begin{array}{l}\text { C2O4 } \\
\text { mass fraction }\end{array}$ & $\begin{array}{l}\text { CO3 } \\
\text { mass fraction }\end{array}$ & $\begin{array}{l}\mathrm{NO} 2 \\
\text { mass fraction }\end{array}$ & $\begin{array}{l}\text { NO3 } \\
\text { mass fraction }\end{array}$ & $\begin{array}{l}\text { OH } \\
\text { mass fraction }\end{array}$ & $\begin{array}{l}\text { SO4 } \\
\text { mass fraction }\end{array}$ & $\begin{array}{l}\text { TcO4 } \\
\text { mass fraction }\end{array}$ & $\begin{array}{l}\text { Temperature } \\
\left({ }^{\circ} \mathrm{C} \text { ) }\right.\end{array}$ \\
\hline RTS114 & 0.02999 & 0.00996 & 0.62295 & 0.14525 & 0.11495 & 0.02595 & 0.02946 & 0.02149 & 35.37 or 70 \\
\hline RTS115 & 0.02991 & 0.02049 & 0.56916 & 0.15546 & 0.12516 & 0.03866 & 0.03967 & 0.02149 & 35.37 or 70 \\
\hline RTS116 & 0.0075 & 0.00001 & 0.39102 & 0.039 & 0.42542 & 0.016 & 0.12063 & 0.00042 & 35.37 or 70 \\
\hline RTS117 & 0.02999 & 0.02049 & 0.33216 & 0.20656 & 0.27016 & 0.04099 & 0.07817 & 0.02149 & 35.37 or 70 \\
\hline RTS118 & 0.02999 & 0.02049 & 0.34018 & 0.18248 & 0.27818 & 0.04099 & 0.08619 & 0.02149 & 35.37 or 70 \\
\hline RTS119 & 0.03 & 0.00001 & 0.76873 & 0.039 & 0.12083 & 0.041 & 0.00001 & 0.00042 & 35.37 or 70 \\
\hline RTS120 & 0.02999 & 0.01495 & 0.68989 & 0.08399 & 0.11789 & 0.02889 & 0.0129 & 0.02149 & 35.37 or 70 \\
\hline RTS121 & 0.02999 & 0.02049 & 0.56736 & 0.12156 & 0.18636 & 0.03436 & 0.01837 & 0.02149 & 35.37 or 70 \\
\hline RTS122 & 0.03 & 0.02048 & 0.34753 & 0.13666 & 0.39046 & 0.01991 & 0.03347 & 0.0215 & 35.37 or 70 \\
\hline RTS123 & 0.0075 & 0.00001 & 0.66902 & 0.09266 & 0.1933 & 0.016 & 0.00001 & 0.0215 & 35.37 or 70 \\
\hline RTS124 & 0.02482 & 0.02049 & 0.68982 & 0.08392 & 0.11782 & 0.02882 & 0.01283 & 0.02149 & 35.37 or 70 \\
\hline RTS125 & 0.02999 & 0.01985 & 0.68249 & 0.10869 & 0.11049 & 0.02149 & 0.0055 & 0.02149 & 35.37 or 70 \\
\hline RTS126 & 0.0075 & 0.00001 & 0.31872 & 0.16098 & 0.44568 & 0.04099 & 0.02569 & 0.00042 & 35.37 or 70 \\
\hline RTS127 & 0.01116 & 0.01596 & 0.74466 & 0.07476 & 0.10866 & 0.01966 & 0.00367 & 0.02149 & 35.37 or 70 \\
\hline RTS128 & 0.03 & 0.0205 & 0.41096 & 0.21296 & 0.25126 & 0.041 & 0.03291 & 0.00042 & 35.37 or 70 \\
\hline RTS129 & 0.02999 & 0.02049 & 0.48576 & 0.10376 & 0.23276 & 0.04099 & 0.06477 & 0.02149 & 35.37 or 70 \\
\hline
\end{tabular}

Table of design points in terms of anion mass fractions and temperature in ${ }^{\circ} \mathrm{C}$. The simulation results of these design points were fit to the mathematical models. The highlighted rows are the OLH design points. The left value in the temperature column was used in simulations for the lower temperature range and the right for the upper temperature range.

\subsection{The Simulation Environment}

OLI Environmental Software Program (ESP) version 6.5 was used with the private databooks carbonat, newtc and gibbsite in addition to the public databook, to simulate the evaporation of Tc eluate to the endpoint temperature and concentration for each design point. As described in the task plan [1] the evaporation endpoint for apparent solubility (and therefore water mass fraction) is defined to be either the point at which the concentrated eluate reaches $1.0 \mathrm{wt} \%$ insoluble solids or when a major salt first begins to appear, which ever occurs first. For the remaining physical properties (density, heat capacity, viscosity and volume fraction), the endpoint is defined as $0.8 \mathrm{wt} \%$ insoluble solids or when the equilibrium constant of a major salt $\left(\mathrm{K}_{\text {major salt }}\right)$ is equal to 0.80 times its solubility product for the mixture $\left(\mathrm{K}_{\text {major salt }}=0.8 * \mathrm{~K}_{\mathrm{sp} \text {-mixture }}\right)$.

Because no volatiles are present, and Tc itself was demonstrated to be non-volatile during evaporation [12], a flash calculation was used to simulate the evaporator. A flow diagram of the simulation is shown in Figure 3-1. The effluent (from Cs ion exchange column) and air stream (representing air in-leakage due to the vacuum existing in the evaporator) are fed at $25^{\circ} \mathrm{C}$ and $1 \mathrm{~atm}$. to a (separator) block representing the evaporator. Some vapor mole fraction is removed from the evaporator at a fixed operating temperature of $70^{\circ} \mathrm{C}$ and the corresponding equilibrium pressure (simulation results were between 0.158-0.305 atm. at the endpoints). The overhead and bottoms are each sent to (mixer) blocks and their equilibrium states calculated at $1 \mathrm{~atm}$. and the endpoint temperature. The physical properties of the resulting product stream are fit the mathematical expression. 
Figure 3-1. OLI/ESP Tc Eluate Evaporation Flowsheet

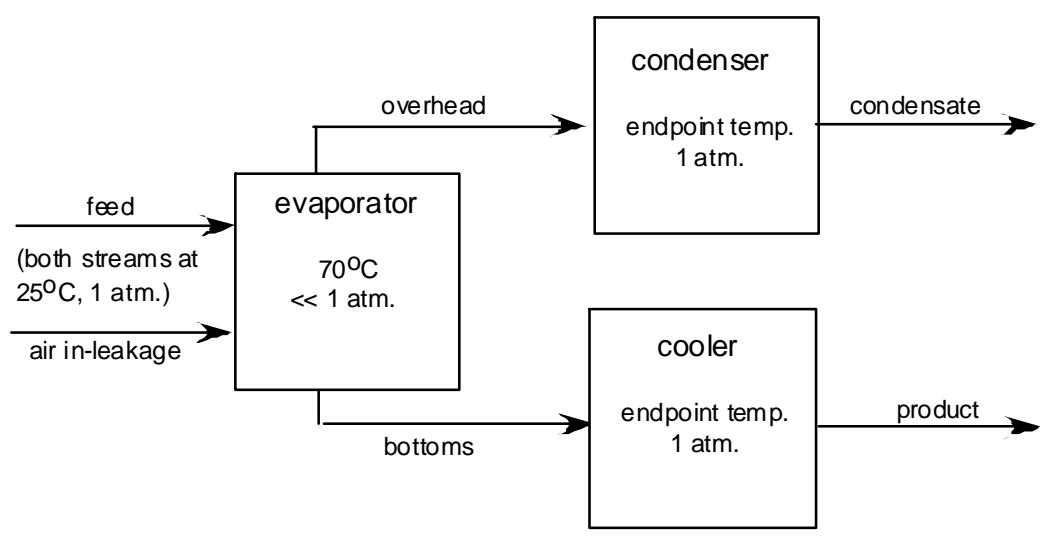

A simplified OLI/ESP chemistry model was used to represent the Tc eluate in the simulations of the design points. As described in Sec. $3.1, \mathrm{Na}^{+}$and $\mathrm{K}^{+}$were the overwhelmingly dominant cations in the actual eluate samples, so no other cations were included as input to the chemistry model. Also, because $\mathrm{Na}^{+}$was never less than $95.3 \mathrm{~mole} \%, \mathrm{Na}^{+}$was fixed at $97.5 \mathrm{~mole} \%$ of the total cations for all design points $\left(\mathrm{K}^{+}\right.$was $2.5 \mathrm{~mole} \%)$. The only other species included as inputs were the eight significant anions. A simplified OLI/ESP chemistry model was based on these 10 input species. Finally, water was added so that each evaporator feed stream (design point) contained 1.0wt\% total solids.

Heat capacities cannot be determined by OLI/ESP (version 6.5) directly and were calculated using enthalpy-temperature plots. These were created from OLI/ESP simulations done at the endpoint composition over $\pm 2^{\circ} \mathrm{C}$ range about the endpoint temperature in $0.2^{\circ} \mathrm{C}$ increments. A similar series of simulations was run over a temperature range of 15 to $75^{\circ} \mathrm{C}$ in one degree increments to provide a broad view of the behavior of the heat capacity. Figure 4-1 and Figure 4-2 in Sec. 4.2 are examples of the $\pm 2^{\circ} \mathrm{C}$ plots.

An Excel macro was written to automate the iterations of the simulations to their endpoints by estimating a new water fraction for the next iteration based on the previous simulations of the design point. A similar macro was written to automate the heat capacity simulations used to create enthalpy-temperature plots. A description and example validation are included in Appendix C.

Prior to simulating the design points, the effect of the relative flow rates of the feed to the air in-leakage on the product composition was examined by running three preliminary simulations. The range of actual evaporator feed rates was calculated to be from 335 to about $1900 \mathrm{~L} / \mathrm{hr}$, based on a $17 \mathrm{wt} \%$ Na loading in the glass at a rate of $30 \mathrm{M}$ tons of glass per day and the eluate/effluent compositions of the $\mathrm{Tc}$ ion exchange lab samples. Three simulations were done, two using a fixed air in-leakage rate of $135 \mathrm{~mol} / \mathrm{hr}$ [13] with corresponding feed rates of 335 and $1700 \mathrm{~L} / \mathrm{hr}$, and a third with no air in-leakage at a feed rate of $1700 \mathrm{~L} / \mathrm{hr}$ (this extreme case goes well beyond the calculated upper bound feed to air in-leakage ratio of $1900 \mathrm{~L} / 135 \mathrm{~mol}$ ). The analytical results of the Tc eluate sample for tank AN-102 were used as the feed composition for all three runs (the OLI/ESP chemistry model was expanded to included all species present above the minimum detection limits). The results listed in Table 3-4 show a minimal effect on the concentrated eluate composition due to variations in the feed to air in-leakage ratio. Therefore, the design point simulations were run at a fixed feed to air in-leakage flow ratio of $1700 \mathrm{~kg} / \mathrm{hr}$ to $135 \mathrm{~mol} / \mathrm{hr}$ (as opposed to $1700 \mathrm{~L} / \mathrm{hr}$ mentioned above, OLI/ESP works in terms moles or mass, not molarity, but the waste feed specific gravity is very close to 1 at $25^{\circ} \mathrm{C}$ ). 
Table 3-4. Effect of Feed to Air In-leakage Flow Ratio on Product Composition

\begin{tabular}{|c|c|c|c|c|c|}
\hline Species & $\begin{array}{l}385 \mathrm{~L} / \mathrm{hr} \text { Eluate } \\
\text { (mass fraction) }\end{array}$ & $\begin{array}{l}\text { 1700L/hr Eluate } \\
\text { (mass fraction) }\end{array}$ & $\begin{array}{c}\text { 1700L/hr Eluate with no air } \\
\text { in-leakage } \\
\text { (mass fraction) }\end{array}$ & $\begin{array}{l}\text { Percent Difference } \\
(1700-385) / 385 * 100\end{array}$ & $\begin{array}{c}\text { Percent Difference } \\
\text { (no air in leakage- } \\
385 \text { ) } / 385 * 100\end{array}$ \\
\hline $\mathrm{OH}$ & 0.0177 & 0.0182 & 0.0183 & 2.4060 & 3.1102 \\
\hline $\mathrm{CO} 3$ & 0.1364 & 0.1358 & 0.1357 & -0.4498 & -0.5817 \\
\hline $\mathrm{HCO} 3$ & $2.15 \mathrm{E}-05$ & $2.09 \mathrm{E}-05$ & $2.07 \mathrm{E}-05$ & -2.8381 & -3.6447 \\
\hline $\mathrm{H}$ & $6.75 \mathrm{E}-16$ & $6.59 \mathrm{E}-16$ & $6.55 \mathrm{E}-16$ & -2.3835 & -3.0610 \\
\hline $\mathrm{NO} 2$ & 0.1130 & 0.1131 & 0.1131 & 0.0351 & 0.0454 \\
\hline $\mathrm{NaHCO} 3$ & $5.00 \mathrm{E}-06$ & $4.86 \mathrm{E}-06$ & $4.82 \mathrm{E}-06$ & -2.7278 & -3.5033 \\
\hline NANO3 & 0.0012 & 0.0012 & 0.0012 & 0.1424 & 0.1845 \\
\hline $\mathrm{AL}(\mathrm{OH}) 3$ & 0.0163 & 0.0161 & 0.0161 & -1.0697 & -1.3833 \\
\hline $\mathrm{AL}(\mathrm{OH}) 4$ & 0.0092 & 0.0094 & 0.0095 & 2.4303 & 3.1420 \\
\hline $\mathrm{K}$ & 0.0139 & 0.0139 & 0.0139 & 0.0327 & 0.0421 \\
\hline $\mathrm{K} 2 \mathrm{SO} 4$ & 0.0023 & 0.0023 & 0.0023 & 0.0929 & 0.1196 \\
\hline $\mathrm{NA} 2 \mathrm{CO} 3$ & 0.0614 & 0.0612 & 0.0612 & -0.2999 & -0.3881 \\
\hline NA & 0.3161 & 0.3162 & 0.3163 & 0.0348 & 0.0451 \\
\hline NA2SO4 & 0.0089 & 0.0089 & 0.0089 & 0.1560 & 0.2015 \\
\hline $\mathrm{NO} 3$ & 0.1345 & 0.1346 & 0.1346 & 0.0346 & 0.0449 \\
\hline $\mathrm{C} 2 \mathrm{O} 4$ & 0.0286 & 0.0286 & 0.0285 & -0.3381 & -0.4371 \\
\hline $\mathrm{SO} 4$ & 0.1072 & 0.1072 & 0.1072 & 0.0260 & 0.0336 \\
\hline $\mathrm{TcO} 4$ & 0.0127 & 0.0127 & 0.0127 & 0.0351 & 0.0454 \\
\hline $\mathrm{NA} 2 \mathrm{C} 2 \mathrm{O} 4$ & 0.0204 & 0.0205 & 0.0206 & 0.8344 & 1.0791 \\
\hline Water mass & 0.8637 & 0.8637 & 0.8637 & -0.0022 & -0.0028 \\
\hline Density $(\mathrm{g} / \mathrm{L})$ & 1109.6700 & 1109.6500 & 1109.6500 & -0.0018 & -0.0018 \\
\hline $\mathrm{pH}$ & 13.1690 & 13.1796 & 13.1826 & 0.0805 & 0.1033 \\
\hline
\end{tabular}

\section{Results}

It has already been mentioned that the precipitation of three different hydrated forms of sodium carbonate $\left(\mathrm{Na}_{2} \mathrm{CO}_{3} \cdot 1 \mathrm{H}_{2} \mathrm{O}, \mathrm{Na}_{2} \mathrm{CO}_{3} .7 \mathrm{H}_{2} \mathrm{O}\right.$, and $\left.\mathrm{Na}_{2} \mathrm{CO}_{3} \cdot 10 \mathrm{H}_{2} \mathrm{O}\right)$ made it necessary to split the temperature range into two regions $\left(20-35.37^{\circ} \mathrm{C}\right.$ and $\left.35.37-70^{\circ} \mathrm{C}\right)$, and physical property expressions developed for each region.

The temperature ranges for which the different hydrated forms of sodium carbonate are stable are defined in the OLI databooks, and are prohibited from precipitating outside this defined range by the OLI/ESP software. Mono-hydrated sodium carbonate precipitates between $20-32^{\circ} \mathrm{C}$, deca-hydrated sodium carbonate between $32-35.37^{\circ} \mathrm{C}$, and hepta-hydrated sodium carbonate from 35.37 to beyond $70^{\circ} \mathrm{C}$. Heptahydrated sodium carbonate has an inverse solubility (decreasing solubility with increasing temperature), while the other two forms display a typical solubility. This opposing solubility behavior among the three hydrated forms of sodium carbonate salt are difficult to capture accurately in a single expression over the entire temperature range. Therefore, the temperature range was divided to include the mono- and decahydrated sodium carbonates in one range, $20-35.37^{\circ} \mathrm{C}$, and the hepta-hydrated form in other, $35.37-70^{\circ} \mathrm{C}$.

This division of the temperature range greatly improved the model fits for the $35.37-70^{\circ} \mathrm{C}$ range, as all the design points had an endpoint of hepta-hydrated sodium carbonate in this range (i.e. there were no other major salts precipitating, and the insoluble solids were always less than $1 \mathrm{wt} \%$ as hepta-hydrated sodium carbonate began to precipitate). However, the model fits in the $20-35.37^{\circ} \mathrm{C}$ range did not improve, again due to the complicated nature of the precipitating species as described below.

\subsection{Simulation Results}

For the lower temperature ranges, 45 of the 129 design points had a $0.8 \mathrm{wt} \%$ insoluble solids endpoints, almost all of which included sodium oxalate (often exclusively), hexa-sodium carbonate disulfate $\left(\mathrm{Na}_{6}\left(\mathrm{SO}_{4}\right)_{2} \mathrm{CO}_{3}\right)$ and, rarely, gibbsite and sodium sulfate. The remaining 84 points had a major salt endpoint. At $20^{\circ} \mathrm{C}$, the endpoint was often either deca-hydrated sodium carbonate or potassium pertechnetate, each occurring with equal frequency. There was one case of a sodium nitrite endpoint for which the nitrite concentration in the feed was very high and that of the carbonate and oxalate very low. Occasionally the endpoint was $1 \mathrm{wt} \%$ insoluble solids. At $35.37^{\circ} \mathrm{C}$ the endpoint was often either potassium pertechnetate or $1.0 \mathrm{wt} \%$ insoluble solids, and occasionally hepta-hydrated sodium carbonate. The increase 
in the $1.0 \mathrm{wt} \%$ insoluble solids endpoints at this higher temperature was due to the appearance of $\mathrm{Na}_{6}\left(\mathrm{SO}_{4}\right)_{2} \mathrm{CO}_{3}$, which is stable only above $30^{\circ} \mathrm{C}$. At intermediate temperatures (the OHL points not used directly in the model fit) with moderate carbonate levels, the endpoint tended to be either pertechnetate or $0.8 \mathrm{wt} \%$ insoluble solids, depending the whether $\mathrm{Na}_{6}\left(\mathrm{SO}_{4}\right)_{2} \mathrm{CO}_{3}$ was precipitating (i.e. below $30^{\circ} \mathrm{C}$, a pertechnetate endpoint with no $\mathrm{Na}_{6}\left(\mathrm{SO}_{4}\right)_{2} \mathrm{CO}_{3}$, above $30^{\circ} \mathrm{C} 1 \mathrm{wt} \%$ solids endpoint with $\mathrm{Na}_{6}\left(\mathrm{SO}_{4}\right)_{2} \mathrm{CO}_{3}$ precipitation). At higher carbonate levels, either hepta- and deca-hydrated sodium carbonate precipitated, depending on the temperature.

The salts considered to be major were the hydrated forms of sodium carbonate $\left(\mathrm{NaCO}_{3} \cdot \mathrm{H}_{2} \mathrm{O}, \mathrm{NaCO}_{3} \cdot 7 \mathrm{H}_{2} \mathrm{O}\right.$, and $\mathrm{NaCO}_{3} \cdot 10 \mathrm{H}_{2} \mathrm{O}$ ), the sodium and potassium salts of nitrate and nitrite, and potassium pertechnetate $\left(\mathrm{KTcO}_{4}\right)$. The only potassium salt observed to precipitate (rarely) was $\mathrm{KTcO}_{4}$. Sodium pertechnetate has a higher solubility than potassium pertechnetate, and therefore never observed to precipitate.

At the completion of the evaporation simulations, the temperature of the eluate at each of the endpoint compositions was varied by $\pm 2.0^{\circ} \mathrm{C}$ about the endpoint temperature in increments of $0.2^{\circ} \mathrm{C}$, to generate enthalpy-temperature plots for heat capacity calculations. In a large majority of cases the enthalpytemperature plots produced straight lines, though there were instances of discontinuities. This occurred when a major salt began to precipitate out with the change in temperature (such as $\mathrm{Na}_{2} \mathrm{CO}_{3} .10 \mathrm{H}_{2} \mathrm{O}$ and $\mathrm{Na}_{6}\left(\mathrm{SO}_{4}\right)_{2} \mathrm{CO}_{3}$ ) causing a change in slope or break in the curve as shown in Figure 4-1 (for run number TR0026). In these cases, the heat capacity was taken to be the slope of the curve prior to precipitation since this is the region of interest for the models. Figure 4-2 shows the portion of the curve in Figure 4-1used to determine the heat capacity.

Figure 4-1. Full Enthalpy Curve

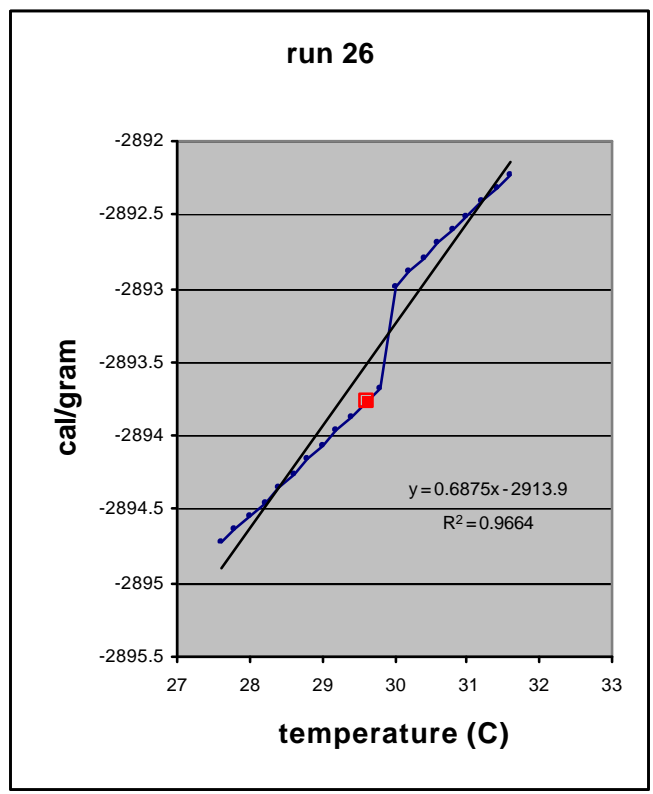

Precipitation of $\mathrm{Na} 6\left(\mathrm{SO}_{4}\right)_{2} \mathrm{CO}_{3}$ occurs at $30^{\circ} \mathrm{C}$, causing a discontinuity in the enthalpy vs. temperature plot at that point. A linear fit is also shown on the plot.
Figure 4-2. Portion of Enthalpy Curve used for Heat Capacity Calculation

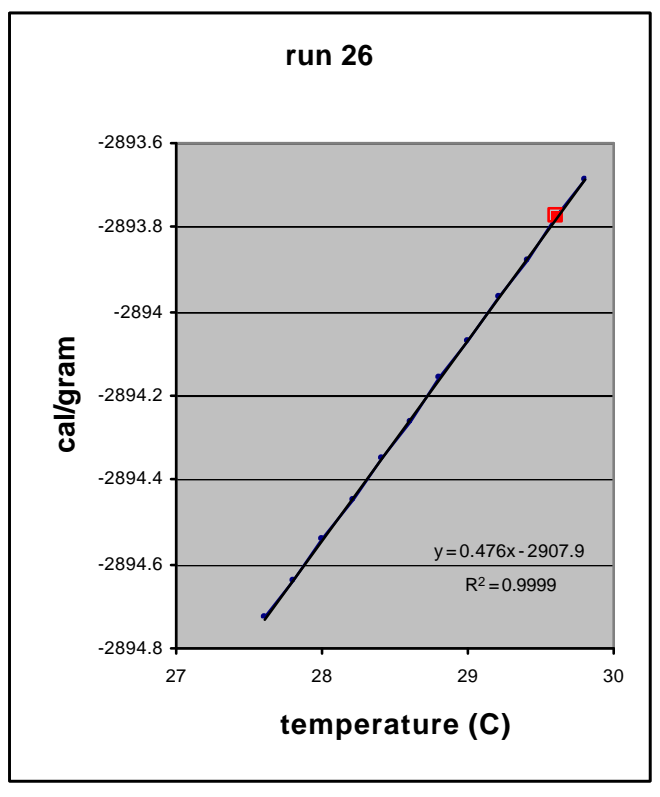

The portion of the curve in Figure 4-1 prior to precipitation of $\mathrm{Na} 6\left(\mathrm{SO}_{4}\right)_{2} \mathrm{CO}_{3}$ is shown above. The slope of this curve is used as heat capacity. A linear fit is also shown on the plot. 


\subsection{The Mathematical Models}

The simulation results (responses) were fit to the trial mathematical forms described in Sec. 3.2 and in "A Statistically Designed Test Matrix for a Computer Study of Tc Eluate Solubility" [2], which is included in Appendix D. The coefficients to the models were calculated in JMP ${ }^{\circledR}$ using the "Fit Model" platform which uses least squares linear regression, with the exception of the non-linear form used for viscosity, for which the "Fit nonlinear" platform in JMP ${ }^{\circledR}$ was used. The physical properties represented by the mathematical models are water mass fraction (WMF, mass water/total mass), solubility (1000* mass soluble solids/mass water $=1000 *[1-\mathrm{WMF}] / \mathrm{WMF}$ ), volume fraction (volume of concentrated Tc eluate /volume feed to Tc eluate evaporator), density (grams/liter), heat capacity $\left(\mathrm{cal} / \mathrm{g} /{ }^{\circ} \mathrm{C}\right)$, and viscosity $(\mathrm{cP})$. A non-linear form of viscosity was fit using the "non-linear" platform of JMP ${ }^{\circledR}$.

The success of a model was determined by its ability to predict a physical property as calculated by the OLI/ESP simulation for each of the 41 OLH design points (points not used in the equation fits). This was measured as the percent difference between the predicted and simulated values ([simulation prediction $] /(\text { prediction })^{*} 100$ ). Table 4-1 and Table 4-2 list the statistics for the percent difference between the model predictions and the OLI/ESP simulation values for the 41 OLH (Orthogonal Latin Hypercube) design points. Rows labeled 16 and 16T represent fits to two equations (Eqs. 17 and 18) that are both linear in composition using the 16 design points, where the equation for 16T includes temperature cross terms. Rows labeled 88 and $88 \mathrm{~T}$ are fits of exactly the same forms (Eqs. 17 and 18) using 88 design points. Rows labeled 88C and 88TC (equations (19) and (20)) represent fits to equations that include both $1^{\text {st }}$ and $2^{\text {nd }}$ order composition terms using the set of 88 design points, where the equation for $88 \mathrm{~T}$ also includes temperature cross terms.

The linear fit for viscosity was consistently poorer than those of other physical properties. Therefore, in addition to the polynomial forms, viscosity was fit to a non-linear form commonly used for pure fluids (Vogel) using the responses of the 88 design points, which greatly improved the fit. The Vogel form expresses the viscosity as an exponential function of temperature, and it is not surprising that the polynomial models were not able to capture this behavior.

Ideally, the mean should be zero. Mean values far from zero relative to the error in the mean (say more than 2 times the error in the mean) would suggest a bias in the model. Taken together, the mean and error in the mean give a measure of the model's accuracy. For example, the estimated mean for the density fit of Eq. 5 in the $35.37-70^{\circ} \mathrm{C}$ range (Table $4-2$, using 88 design points with no temperature terms) was $0.52 \%$, with an error in the estimated mean of $0.29 \%$. The positive mean might indicate a slight bias of the model to under-predict the simulated values. However, the error in the mean is of the same order of magnitude, so it is difficult to make a statistical distinction between the estimated mean and zero.

The standard deviation is of course a measure of the precision about the estimated mean, the smaller it is the better. For the density mentioned above, the standard deviation about the estimated mean is $1.9 \%$. These three measures taken together (mean, error in mean, and standard deviation) indicate the density model is very successful at predicting the simulation results.

The columns labeled min and max give the extreme values (minimum and maximum) of the percent difference. Again, for the same density, the highlighted model did not over-predict any of the OLH design points by more that $3.2 \%$, nor did it under-predict any by more than $4.3 \%$.

The highlighted rows correspond to the models felt to have the best combination of accuracy and precision. Where there is little difference in performance, the simpler form was chosen. The equations corresponding to the highlighted rows are given below. 
WSRC-TR-2002-00319 Rev. 0

SRT-RPP-2002-00171

Statistical Comparison in terms of Percent Difference between Model and Simulation Results for OLH Design Points

Table 4-1 20-35.37 ${ }^{\circ} \mathrm{C}$

Table 4-2 $35.37-70^{\circ} \mathrm{C}$

(highlighted rows used for model fits)

(highlighted rows used for model fits)

\begin{tabular}{|c|c|c|c|c|c|}
\hline model & mean & $\begin{array}{r}\text { Error in } \\
\text { mean }\end{array}$ & $\begin{array}{l}\text { std } \\
\text { dev }\end{array}$ & $\min$ & $\operatorname{Max}$ \\
\hline \multicolumn{6}{|c|}{$\begin{array}{c}\text { WMF } \\
\text { (at saturation) }\end{array}$} \\
\hline 16 & -10.5 & 2.9 & 18.8 & -47.0 & 47.3 \\
\hline $16 \mathrm{~T}$ & -11.3 & 2.7 & 17.5 & -46.6 & 46.9 \\
\hline 88 & -13.4 & 2.4 & 15.6 & -45.4 & 20.1 \\
\hline $88 \mathrm{~T}$ & -13.9 & 2.4 & 15.3 & -46.7 & 21.7 \\
\hline $88 \mathrm{C}$ & -91.1 & 4.6 & 29.6 & -98.5 & 84.2 \\
\hline 88TC & -90.8 & 3.4 & 21.7 & -98.8 & 33.7 \\
\hline \multicolumn{6}{|c|}{$\begin{array}{c}\text { Solubility } \\
\text { (at saturation) }\end{array}$} \\
\hline 16 & 21.9 & 7.5 & 48.0 & -62.2 & 181 \\
\hline $16 \mathrm{~T}$ & 21.9 & 7.3 & 46.8 & -61.1 & 179 \\
\hline 88 & 29.1 & 7.6 & 29.1 & -49.4 & 169 \\
\hline $88 \mathrm{~T}$ & 30.0 & 7.1 & 30.0 & -48.5 & 187 \\
\hline $88 \mathrm{C}$ & -95.8 & 4.6 & 29.6 & -112 & 75.2 \\
\hline 88TC & -85.9 & 14.3 & 91.6 & -115 & 481 \\
\hline \multicolumn{6}{|c|}{$\begin{array}{c}\text { volume fraction } \\
\text { (at } 80 \% \text { saturation) }\end{array}$} \\
\hline 16 & -23.4 & 4.3 & 27.3 & -55.8 & 68.3 \\
\hline $16 \mathrm{~T}$ & -26.3 & 3.8 & 24.2 & -56.0 & 78.5 \\
\hline 88 & -26.4 & 3.0 & 19.1 & -54.4 & 21.8 \\
\hline 88T & -26.6 & 3.5 & 22.3 & -54.5 & 25.7 \\
\hline $88 \mathrm{C}$ & -121 & 16.6 & 106 & -742 & 96.3 \\
\hline $88 \mathrm{TC}$ & -105 & 19.5 & 125 & -796 & 268 \\
\hline \multicolumn{6}{|c|}{$\begin{array}{c}\text { Density } \\
\text { (at } 80 \% \text { saturation) }\end{array}$} \\
\hline 16 & 5.5 & 1.1 & 7.0 & -11.5 & 20.1 \\
\hline $16 \mathrm{~T}$ & 5.6 & 1.0 & 6.5 & -11.4 & 19.4 \\
\hline 88 & 6.3 & 1.0 & 6.6 & -7.9 & 21.6 \\
\hline $88 \mathrm{~T}$ & 6.5 & 0.9 & 6.1 & -8.4 & 18.6 \\
\hline $88 \mathrm{C}$ & -123 & 13.3 & 85.4 & -630 & 4.9 \\
\hline $88 \mathrm{TC}$ & -105 & 8.6 & 55.2 & -306 & 6.3 \\
\hline \multicolumn{6}{|c|}{$\begin{array}{l}\text { heat capacity } \\
\text { (at } 80 \% \text { saturation) }\end{array}$} \\
\hline 16 & 5.5 & 1.1 & 7.0 & -11.5 & 20.1 \\
\hline $16 \mathrm{~T}$ & 5.6 & 1.0 & 6.5 & -11.4 & 19.4 \\
\hline 88 & 6.3 & 1.0 & 6.6 & -7.9 & 21.6 \\
\hline $88 \mathrm{~T}$ & 6.5 & 0.9 & 6.1 & -8.4 & 18.6 \\
\hline $88 \mathrm{C}$ & -123 & 13.3 & 85.4 & -630 & 4.9 \\
\hline 88TC & -105 & 8.6 & 55.2 & -306 & 6.3 \\
\hline \multicolumn{6}{|c|}{$\begin{array}{c}\text { Viscosity } \\
\text { (at } 80 \% \text { saturation) }\end{array}$} \\
\hline 16 & 54.3 & 12.0 & 76.9 & -62.1 & 278 \\
\hline $16 \mathrm{~T}$ & 57.2 & 12.5 & 80.3 & -65.2 & 297 \\
\hline 88 & 44.5 & 10.8 & 68.8 & -53.4 & 197 \\
\hline $88 \mathrm{~T}$ & 44.3 & 9.4 & 60.2 & -57.1 & 199 \\
\hline $88 \mathrm{C}$ & -81.2 & 3.6 & 22.9 & -95.1 & 49.7 \\
\hline 88TC & -47.4 & 49.4 & 316 & -235 & 1915 \\
\hline
\end{tabular}

\begin{tabular}{|c|c|c|c|c|c|}
\hline model & mean & $\begin{array}{r}\text { Error in } \\
\text { mean }\end{array}$ & $\begin{array}{l}\text { std } \\
\text { dev }\end{array}$ & Min & $\operatorname{Max}$ \\
\hline \multicolumn{6}{|c|}{$\begin{array}{c}\text { WMF } \\
\text { (at saturation) }\end{array}$} \\
\hline 16 & -1.51 & 0.86 & 5.5 & -11.9 & 10.8 \\
\hline $16 \mathrm{~T}$ & -1.4 & 0.9 & 5.8 & -16.2 & 12.4 \\
\hline 88 & -2.6 & 0.6 & 3.9 & -11.5 & 5.5 \\
\hline $88 \mathrm{~T}$ & -2.5 & 0.6 & 3.9 & -11.0 & 3.4 \\
\hline $88 \mathrm{C}$ & -134 & 16.0 & 104 & -682 & 150 \\
\hline 88TC & -75.0 & 20.0 & 128 & -301 & 476 \\
\hline \multicolumn{6}{|c|}{$\begin{array}{c}\text { Solubility } \\
\text { (at saturation) }\end{array}$} \\
\hline 16 & 0.83 & 2.0 & 12.9 & -21.7 & 28.9 \\
\hline $16 \mathrm{~T}$ & 1.3 & 2.5 & 15.9 & -23.8 & 51.2 \\
\hline 88 & 4.7 & 1.5 & 9.9 & -13.4 & 28.1 \\
\hline $88 \mathrm{~T}$ & 4.7 & 1.5 & 9.9 & -10.4 & 26.1 \\
\hline $88 \mathrm{C}$ & -88.3 & 3.2 & 20.5 & -95.6 & 8.2 \\
\hline 88TC & -100 & 18.0 & 115 & -692 & 223 \\
\hline \multicolumn{6}{|c|}{$\begin{array}{l}\text { volume fraction } \\
\text { (at } 80 \% \text { saturation) }\end{array}$} \\
\hline 16 & -3.9 & 1.5 & 9.4 & -17.1 & 24.6 \\
\hline $16 \mathrm{~T}$ & -3.7 & 1.5 & 9.6 & -20.2 & 28.5 \\
\hline 88 & -4.9 & 0.87 & 5.6 & -14.8 & 8.4 \\
\hline $88 \mathrm{~T}$ & -4.7 & 0.91 & 5.8 & -16.2 & 10.6 \\
\hline $88 \mathrm{C}$ & -91.8 & 3.5 & 22.3 & -98.1 & 11.9 \\
\hline 88TC & -91.6 & 3.5 & 22.7 & 98.2 & 13.0 \\
\hline \multicolumn{6}{|c|}{$\begin{array}{c}\text { Density } \\
\text { (at } 80 \% \text { saturation) }\end{array}$} \\
\hline 16 & 0.33 & 0.33 & 2.1 & -3.9 & 4.3 \\
\hline $16 \mathrm{~T}$ & 0.30 & 0.34 & 2.2 & -3.9 & 5.0 \\
\hline 88 & 0.52 & 0.29 & 1.9 & -3.2 & 4.3 \\
\hline $88 \mathrm{~T}$ & -0.50 & 0.29 & 1.8 & -3.0 & 4.5 \\
\hline $88 \mathrm{C}$ & -170 & 58.7 & 376 & -961 & 1841 \\
\hline 88TC & -76.4 & 67.3 & 431 & -1657 & 1173 \\
\hline \multicolumn{6}{|c|}{$\begin{array}{l}\text { heat capacity } \\
\text { (at } 80 \% \text { saturation) }\end{array}$} \\
\hline 16 & -1.2 & 0.68 & 4.3 & 10.1 & 7.6 \\
\hline $16 \mathrm{~T}$ & -1.1 & 0.69 & .4 & -10.9 & 8.0 \\
\hline 88 & -2.2 & 0.52 & 3.3 & -7.8 & 5.8 \\
\hline $88 \mathrm{~T}$ & -2.2 & 0.50 & 3.2 & -8.4 & 4.1 \\
\hline $88 \mathrm{C}$ & -110 & 13.8 & 87.8 & 296 & 375 \\
\hline 88TC & -99.9 & 14.9 & 95.4 & -436 & 144 \\
\hline \multicolumn{6}{|c|}{$\begin{array}{c}\text { Viscosity } \\
\text { (at } 80 \% \text { saturation) }\end{array}$} \\
\hline 16 & -6.61 & 4.3 & 27.7 & -43.0 & 52.5 \\
\hline $16 \mathrm{~T}$ & $-6-6$ & 4.8 & 30.4 & -42.9 & 68.9 \\
\hline 88 & -10.1 & 4.0 & 25.5 & -44.5 & 37.3 \\
\hline $88 \mathrm{~T}$ & -9.6 & 4.6 & 29.7 & -49.0 & 47.3 \\
\hline $88 \mathrm{C}$ & -78.7 & 16.7 & 107 & -99.0 & 582 \\
\hline $88 \mathrm{TC}$ & 9.5 & 104 & 666 & -99.0 & 4170 \\
\hline
\end{tabular}

Measure of physical property models in terms of percent difference between model prediction and simulation results for the $41 \mathrm{OLH}$ design points. 


\section{Expressions valid for $35.37-70^{\circ} \mathrm{C}$}

The apparent solubility ( $1 \mathrm{wt} \%$ insoluble solids or precipitation of a major salt) is given as:

$$
\begin{aligned}
& \text { solubility at endpoint conditions }\left(\mathrm{g} \text { solids } / \mathrm{kg} \mathrm{H}_{2} \mathrm{O}\right) \\
& \begin{aligned}
\mathbf{7 2 8} *\left[\mathrm{AlO}_{2}\right]-2,510 *\left[\mathrm{CO}_{4}\right]+492 *\left[\mathrm{CO}_{3}\right]+554 *[\mathrm{NO}]+1,070 *[\mathrm{NO}] \\
\quad+\mathbf{2 , 7 7 0} *[\mathrm{OH}]+827 *[\mathrm{SQ}]+733 *[\mathrm{TcQ}]
\end{aligned}
\end{aligned}
$$

having a mean of $4.7 \%$ and a standard deviation of $9.9 \%$. The concentrations are in terms of the anion mass fraction relative to the total mass of the anions.

The water mass fraction (WMF) is given as:

$$
\begin{aligned}
& \text { WMF at endpoint conditions }\left(\mathrm{g} \mathrm{H}_{2} \mathrm{O} / \mathrm{g}\right. \text { solution) } \\
& =0.498 *\left[\mathrm{AlO}_{2}\right]+1.59 *\left[\mathrm{C}_{2} \mathrm{O}_{4}\right]+0.662 *\left[\mathrm{CO}_{3}\right]+0.624 *\left[\mathrm{NO}_{2}\right]+0.477 *\left[\mathrm{NO}_{3}\right] \\
& \quad-0.03099 *[\mathrm{OH}]+0.575 *\left[\mathrm{SO}_{4}\right]+0.608 *\left[\mathrm{TcO}_{4}\right]
\end{aligned}
$$

having a mean of $-2.60 \%$ and a standard deviation of $3.93 \%$. The concentrations are in terms of the anion mass fraction relative to the total mass of the anions.

The density is given as:

$$
\begin{aligned}
& \text { density at endpoint conditions }(\mathrm{g} / \mathrm{L}) \\
& =1,920 *[\mathrm{AlO}]+1,150 *\left[\mathrm{C}_{2} \mathrm{O}_{4}\right]+1,260 *[\mathrm{CO}]+1,210 *[\mathrm{NO}] \\
& \\
& \quad+1,300 *\left[\mathrm{NO}_{3}\right]+2,470 *[\mathrm{OH}]+1,180 *\left[\mathrm{SO}_{4}\right]+1,060 *[\mathrm{TcO}]
\end{aligned}
$$

having a mean of $0.33 \%$ and a standard deviation of $2.09 \%$. The concentrations are in terms of the anion mass fraction relative to the total mass of the anions.

The heat capacity is given as:

$$
\begin{aligned}
& \text { heat capacity at endpoint conditions }\left(\mathrm{cal} / \mathrm{g} /{ }^{\mathrm{o}} \mathrm{C}\right) \\
& =0.504 *[\mathrm{AlO}]+\mathbf{2 . 7 0} *\left[\mathrm{GO}_{4}\right]+0.656 *[\mathrm{CO}]+0.736 *[\mathrm{NO}] \\
& +0.559 *[\mathrm{NO}]-1.06 *[\mathrm{OH}]+0.667 *[\mathrm{SO}]+1.23 *[\mathrm{TcO}]
\end{aligned}
$$

having a mean of $1.16 \%$ and a standard deviation of $4.32 \%$. The concentrations are in terms of the anion mass fraction relative to the total mass of the anions.

The viscosity is given as:

$$
\begin{aligned}
& \text { viscosity at endpoint conditions (cP) } \\
& =28.5 *\left[\mathrm{AlO}_{3}\right]+37.2 *\left[\mathrm{C}_{2} \mathrm{O}_{4}\right]+3.02 *\left[\mathrm{CO}_{3}\right]+2.41 *\left[\mathrm{NO}_{2}\right] \\
& +1.18 *[\mathrm{NO}]-7.14 *[\mathrm{OH}]+1.00 *[\mathrm{SO}]+22.9 *[\mathrm{TcO}]
\end{aligned}
$$

having a mean of $6.62 \%$ and a standard deviation of and $27.7 \%$. The concentrations are in terms of the anion mass fraction relative to the total mass of the anions. Because of the poor performance of this form of equation for viscosity, the form given by equation (6) below should be used instead. 
The Vogel form for viscosity is given as:

$$
\begin{aligned}
& \text { viscosity at endpoint conditions (cP) } \\
& =\exp \left(\frac{140}{\left(\text { temperature }\left({ }^{\circ} \mathrm{C}\right)+20\right)}-100\right)
\end{aligned}
$$

having a mean of $0.07 \%$ and a standard deviation of $8.62 \%$, and is a function of temperature only. This form should be used in lieu of equation (5).

The volume fraction is given as:

$$
\begin{aligned}
& \text { volume fraction at endpoint conditions } \\
& \text { (concentrated eluate volume / evaporator feed volume at } 20^{\circ} \mathrm{C} \text { and } 1 \mathrm{wt} \% \text { total solids) } \\
& =\mathbf{0 . 0 1 1 7} *\left[\mathrm{AlO}_{2}\right]+\mathbf{0 . 0 3 3 3}^{*}\left[\mathrm{CO}_{4}\right]+\mathbf{0 . 0 1 4 5}^{*}\left[\mathrm{CO}_{3}\right]+\mathbf{0 . 0 1 1 8}^{*}[\mathrm{NO}] \\
& +\mathbf{0 . 0 0 8 0 2}^{*}\left[\mathrm{NO}_{3}\right]-0.00946 *[\mathrm{OH}]+\mathbf{0 . 0 1 0 5}^{*}\left[\mathrm{SO}_{4}\right]+\mathbf{0 . 0 1 5 6}^{*}[\mathrm{TcO}]
\end{aligned}
$$

having a mean of $4.9 \%$ and a standard deviation of $5.58 \%$. The concentrations are in terms of the anion mass fraction relative to the total mass of the anions. The volume fraction is relative to a feed volume containing $1 \mathrm{wt} \%$ total solids at $20^{\circ} \mathrm{C}$.

The volume reduction is given as:

\section{volume reduction at endpoint conditions}

(evaporator feed volume at $20^{\circ} \mathrm{C}$ and $1 \mathrm{wt} \%$ total solids / concentrated eluate volume)

$$
\begin{aligned}
= & 63.7 *\left[\mathrm{AlO}_{2}\right]-86.8 *\left[\mathrm{C}_{2} \mathrm{O}_{4}\right]+67.0 *\left[\mathrm{CO}_{3}\right]+79.0 *[\mathrm{NO}] \\
& +117 *\left[\mathrm{NO}_{3}\right]-258 *[\mathrm{OH}]+103 *[\mathrm{SQ}]+58.9 *[\mathrm{TcQ}]
\end{aligned}
$$

having a mean of $4.19 \%$ and a standard deviation of $6.36 \%$. The concentrations are in terms of the anion mass fraction relative to the total mass of the anions. The volume reduction is relative to a feed volume containing $1 \mathrm{wt} \%$ total solids at $20^{\circ} \mathrm{C}$.

Figure 4-3 - Figure 4-10 below show plots of the simulation physical property (such as solubility) vs. the corresponding model prediction of the physical property for the $35.37-70^{\circ} \mathrm{C}$ range. Two error curves representing $\pm 15 \%$ of the model prediction are included in the plots to quantify the success of the models. If $67 \%$ (1-sigma) of the points lay with the error curves, then the models meet the acceptance criteria. It can be seen that, with the exception of the liner form of viscosity, all, or very nearly all, of the points are within the error curves, and show that the models for the $35.37-70^{\circ} \mathrm{C}$ range exceed the acceptance criteria (again, with the exception of the linear form for viscosity). 
Figure 4-3 Simulation vs. Model Apparent Solubility $35.37-70^{\circ} \mathrm{C}$

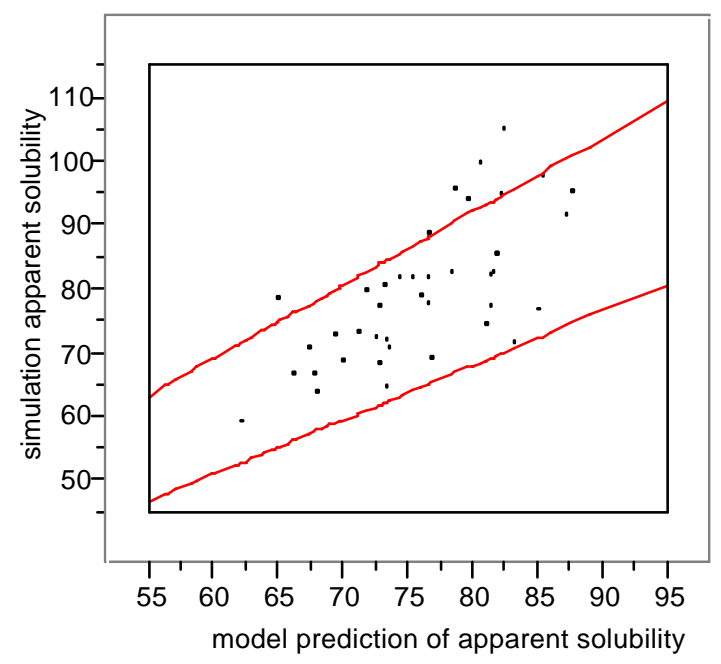

Plot of simulation vs. model apparent solubility of the 41 OLH design points. The two lines indicate the $\pm 15 \%$ error bounds of the model given by Eq. (1).

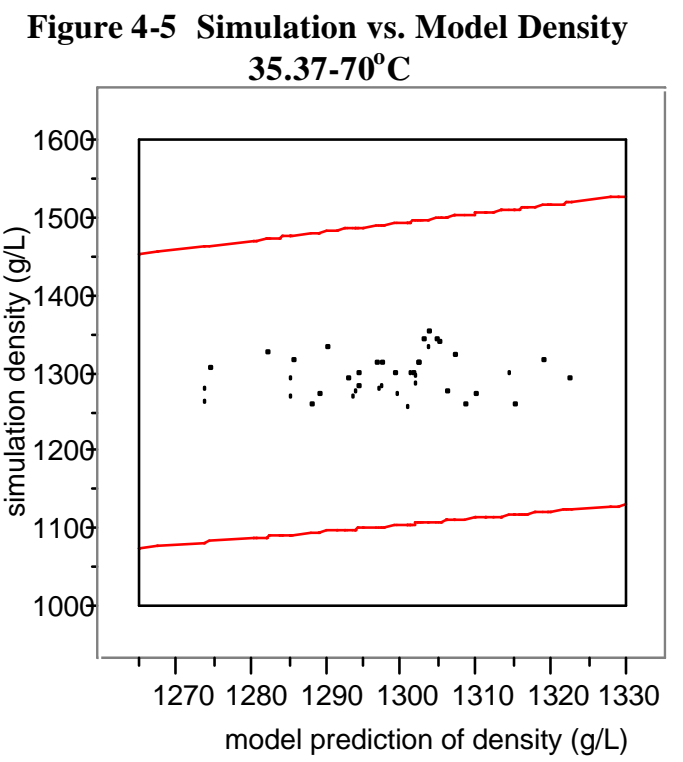

Plot of simulation vs. model density of the $41 \mathrm{OLH}$ design points. The two lines indicate the $\pm 15 \%$ error bounds of the model given by Eq. (3).

\section{Figure 4-4 Simulation vs. Model Water Mass Fraction $35.37-70^{\circ} \mathrm{C}$}

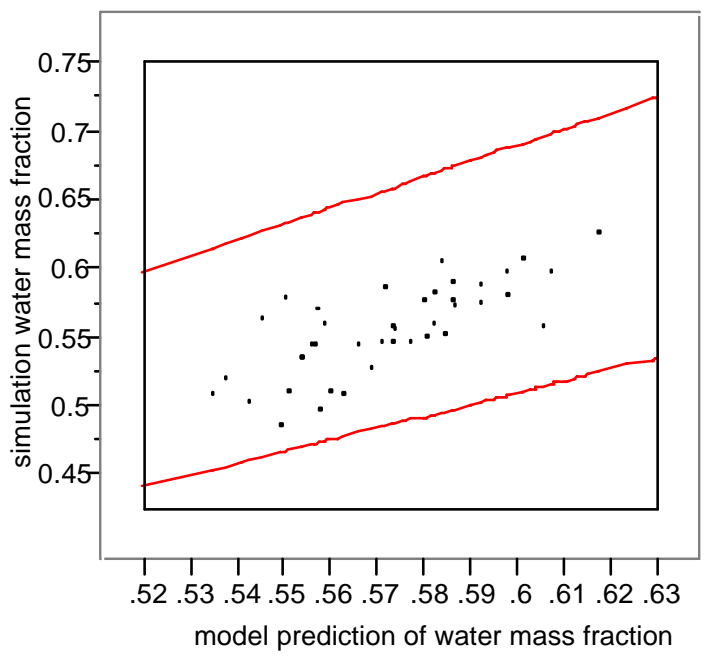

Plot of simulation vs. model water mass fraction of the 41 OLH design points. The two lines indicate the $\pm 15 \%$ error bounds of the model given by Eq. (2).

Figure 4-6 Simulation vs. Model Heat Capacity

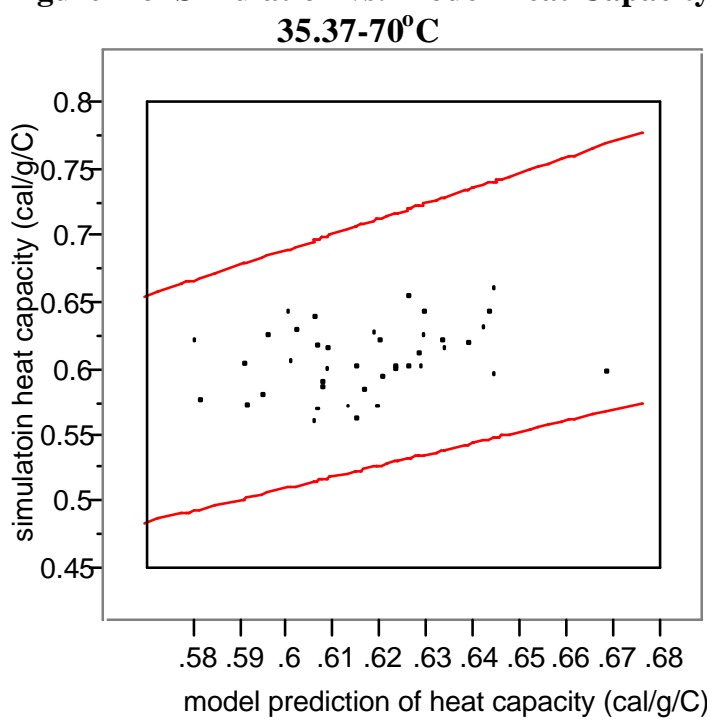

Plot of simulation vs. model heat capacity of the 41 OLH design points. The two lines indicate the $\pm 15 \%$ error bounds of the model given by Eq. (4). 
Figure 4-7 Simulation vs. Linear Model for

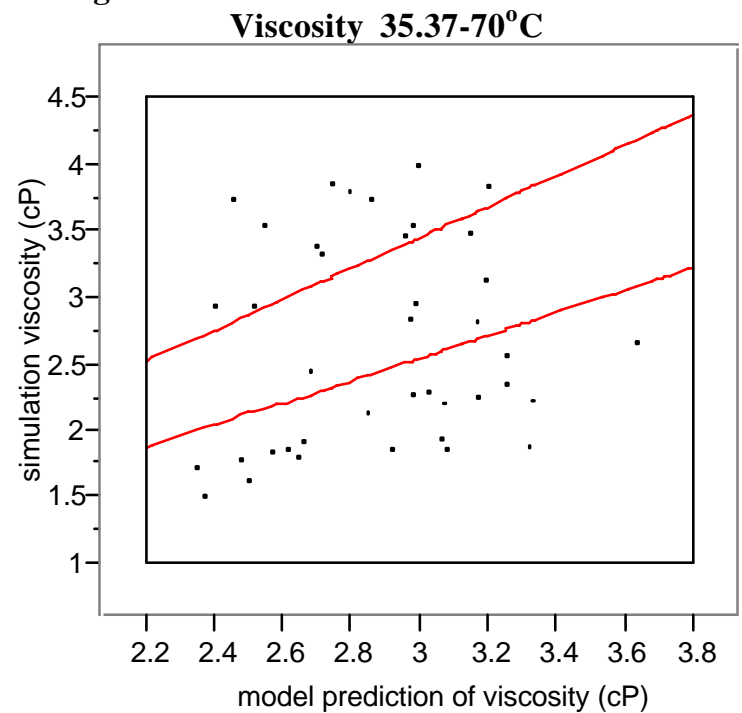

Plot of simulation vs. linear model for viscosity of the $41 \mathrm{OLH}$ design points. The two lines indicate the $\pm 15 \%$ error bounds of the model given by Eq. (5).

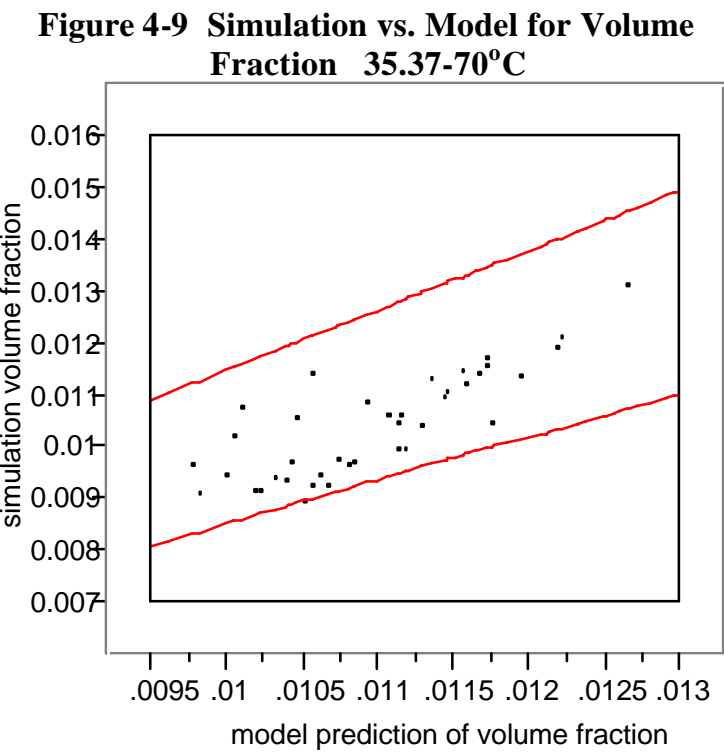

Plot of simulation vs. model volume fraction of the 41 OLH design points. The two lines indicate the $\pm 15 \%$ error bounds of the model given by Eq. (7)

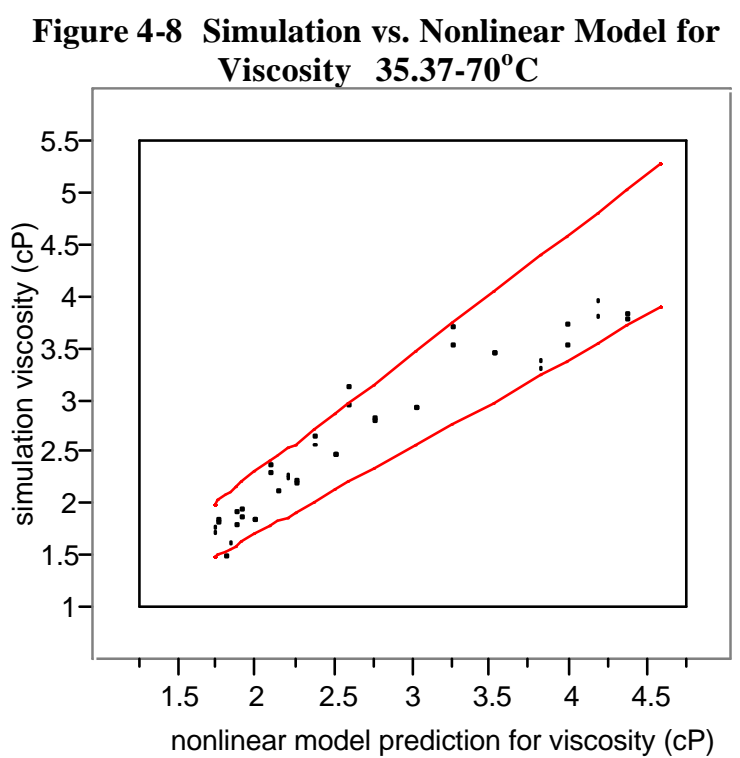

Plot of simulation vs. nonlinear model (Vogel) for viscosity of the 41 OLH design points. The two lines indicate the $\pm 15 \%$ error bounds of the model given by Eq. (6)

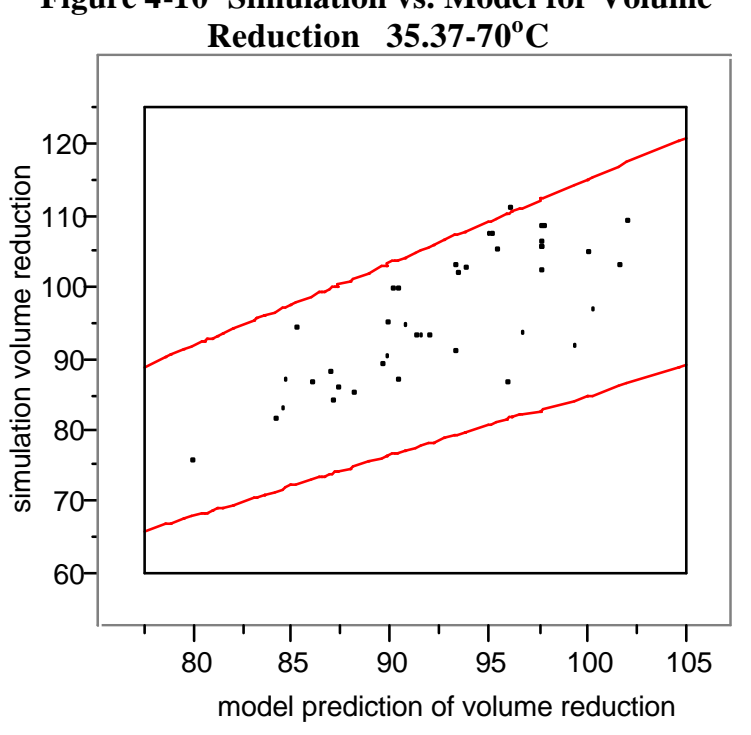

Plot of simulation vs. model volume reduction of the 41 OLH design points. The two lines indicate the $\pm 15 \%$ error bounds of the model given by Eq. (8). 


\section{Expressions valid for $20-35.37^{\circ} \mathrm{C}$}

The apparent solubility (1wt\% insoluble solids or precipitation of a major salt) is given as:

$$
\begin{aligned}
& \text { solubility at endpoint conditions }\left(\mathrm{g} \text { solids } / \mathrm{kg} \mathrm{H}_{2} \mathrm{O}\right) \\
& \begin{aligned}
= & -167,000 *[\mathrm{AlO}]-74,600 *\left[\mathrm{CO}_{4}\right]+12,000 *\left[\mathrm{CO}_{3}\right]+16,300 *[\mathrm{NO}] \\
& +22,900 *\left[\mathrm{NO}_{3}\right]+48,800 *[\mathrm{OH}]+3,160 *\left[\mathrm{SO}_{4}\right]-173,000 *[\mathrm{TcO}]
\end{aligned}
\end{aligned}
$$

having a mean of $21.9 \%$ and a standard deviation of $48 \%$. The concentrations are in terms of the anion mass fraction relative to the total mass of the anions.

The water mass fraction (WMF) is given as:

$$
\begin{aligned}
& \text { WMF at endpoint conditions (grams } \mathrm{H}_{2} \mathrm{O} / \text { grams solution) } \\
& \begin{aligned}
= & 4.62 * \mathrm{AlO}_{2}+4.20 * \mathrm{C}_{2} \mathrm{O}_{4}+0.516 * \mathrm{CO}_{3}+0.451 * \mathrm{NO}_{2}+0.207 * \mathrm{NO}_{3} \\
& -1.58 * \mathrm{OH}+0.668 * \mathrm{SO}_{4}+5.20 * \mathrm{TcO}_{4}
\end{aligned}
\end{aligned}
$$

having a mean of $-10.5 \%$ and a standard deviation of $18.8 \%$. The concentrations are in terms of the anion mass fraction relative to the total mass of the anions.

The density is given as:

$$
\begin{aligned}
& \text { density at endpoint conditions }(\mathrm{g} / \mathrm{L}) \\
& =-1,520 *[\mathrm{AlO}]+762 *\left[\mathrm{C}_{2} \mathrm{O}_{4}\right]+1,310 *[\mathrm{CO}]+1,490 *[\mathrm{NO}] \\
& \quad+1,620 *[\mathrm{NO}]+2,510 *[\mathrm{OH}]+1,100 *[\mathrm{SO}]-2,670 *[\mathrm{TcO}]
\end{aligned}
$$

having a mean of $5.5 \%$ and a standard deviation of $7.0 \%$. The concentrations are in terms of the anion mass fraction relative to the total mass of the anions.

The heat capacity is given as:

$$
\begin{aligned}
& \text { heat capacity at endpoint conditions }\left(\mathrm{cal} / \mathrm{g} /{ }^{\circ} \mathrm{C}\right) \\
& =3.57 *[\mathrm{AlO}]+3.17 *\left[\mathrm{CO}_{4}\right]+0.687 *[\mathrm{CO}]+0.528 *[\mathrm{NO}] \\
& +0.349 *\left[\mathrm{NO}_{3}\right]-1.62 *[\mathrm{OH}]+0.738 *\left[\mathrm{SO}_{4}\right]+4.76 *[\mathrm{TcO}]
\end{aligned}
$$

having a mean of $-11.5 \%$ and a standard deviation of $13.6 \%$. The concentrations are in terms of the anion mass fraction relative to the total mass of the anions.

The viscosity is given as:

$$
\begin{aligned}
& \text { viscosity at endpoint conditions }(\mathrm{cP}) \\
& =28.5 *[\mathrm{AlO}]+37.2 *\left[\mathrm{C}_{2} \mathrm{O}_{4}\right]+3.02 *\left[\mathrm{CO}_{3}\right]+2.41 *[\mathrm{NO}] \\
& +1.18 *[\mathrm{NO}]-7.14 *[\mathrm{OH}]+1.00 *\left[\mathrm{SO}_{4}\right]+22.9 *[\mathrm{TcO}]
\end{aligned}
$$

having a mean of $44.5 \%$ and a standard deviation of $68.8 \%$. The concentrations are in terms of the anion mass fraction relative to the total mass of the anions. 
The alternative form non-linear form for viscosity (Vogel) is given below:

$$
\begin{aligned}
& \text { viscosity at endpoint conditions }(\mathrm{cP}) \\
& =\exp \left(000174 * \operatorname{density}(\mathrm{g} / \mathrm{L})-\frac{0.0181 * \operatorname{density}(\mathrm{g} / \mathrm{L})}{\left(\operatorname{temperature}\left({ }^{\circ} \mathrm{C}\right)+5\right)}\right)
\end{aligned}
$$

having a mean of $54.8 \%$ and a standard deviation of $65.7 \%$, and is a function of temperature and density only. Note that unlike its corresponding equation (6) for the $35.37-70^{\circ} \mathrm{C}$ range, this equation includes density terms. The measured density of the sample should be used as opposed to that calculated by equation (11).

The volume fraction is given as:

volume fraction at endpoint conditions

(concentrated eluate volume / evaporator feed volume at $20^{\circ} \mathrm{C}$ and $1 \mathrm{wt} \%$ total solids)

$=0.0626 *\left[\mathrm{AlO}_{2}\right]+0.0720 *\left[\mathrm{CO}_{4}\right]+0.0239 *\left[\mathrm{CO}_{3}\right]+0.00626 *\left[\mathrm{NO}_{2}\right]$

$+0.00291 *\left[\mathrm{NO}_{3}\right]-0.0808 *[\mathrm{OH}]+0.0149 *[\mathrm{SO}]+0.0649 *\left[\mathrm{TcO}_{4}\right]$

having a mean of $-26 \%$ and a standard deviation of $19 \%$. The concentrations are in terms of the anion mass fraction relative to the total mass of the anions. The volume fraction is relative to a feed volume containing $1 \mathrm{wt} \%$ total solids at $20^{\circ} \mathrm{C}$.

The volume reduction is given as:

volume reduction at endpoint conditions

(evaporator feed volume at $20^{\circ} \mathrm{C}$ and $1 \mathrm{wt} \%$ total solids / concentrated eluate volume)

$=-888 *[\mathrm{AlO}]-438 *\left[\mathrm{GO}_{4}\right]+\mathbf{8 6 . 0} *[\mathrm{CO}]+142 *[\mathrm{NO}]$

$+198 *[\mathrm{NO}]+636 *[\mathrm{OH}]+32.8 *\left[\mathrm{SO}_{4}\right]-1,290 *[\mathrm{TcQ}]$

having a mean of $23 \%$ and a standard deviation of $29 \%$. The concentrations are in terms of the anion mass fraction relative to the total mass of the anions. The volume reduction is relative to a feed volume containing $1 \mathrm{wt} \%$ total solids at $20^{\circ} \mathrm{C}$.

Figure 4-11 - Figure 4-18 below show plots of the simulation physical property (such as solubility) vs. the corresponding model prediction of the physical property for the $20-35.37^{\circ} \mathrm{C}$ range. Two error curves representing $\pm 15 \%$ of the model prediction are included in the plots to quantify the success of the models. If $67 \%$ (1-sigma) of the points lay with the error curves, then the models meet the acceptance criteria. Unlike the corresponding figures (Figure 4-3 - Figure 4-10) for the $35.37-70^{\circ} \mathrm{C}$ range, a significant number of the points fall outside the error curves (with the exception of density), and many plots appear as a shotgun blast, suggesting no significant correlation to the models. Figure 4-17 does appear to show some correlation, but with very poor accuracy. 
Figure 4-11 Simulation vs. Model Apparent Solubility $20-35.37^{\circ} \mathrm{C}$

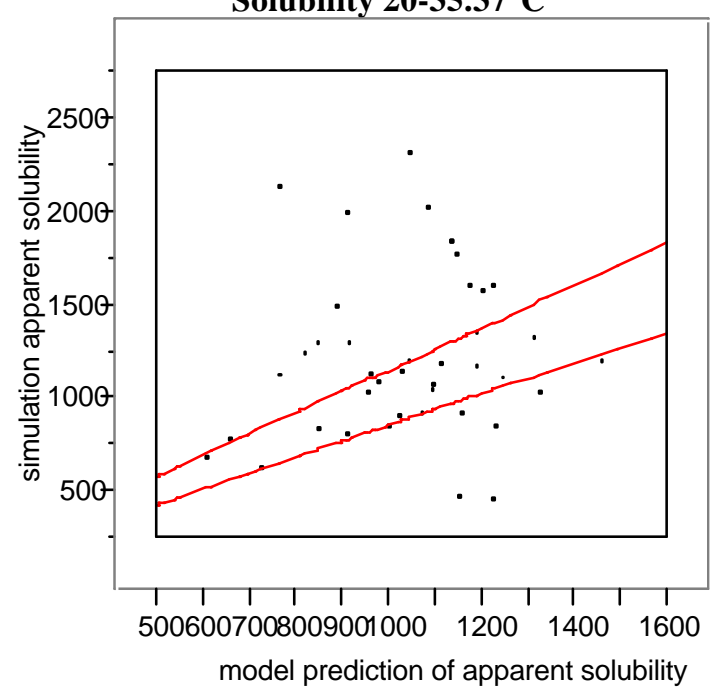

Plot of simulation vs. model apparent solubility of the 41 OLH design points. The two lines indicate the $\pm 15 \%$ error bounds of the model given by Eq. (9).

Figure 4-13 Simulation vs. Model Density 20-

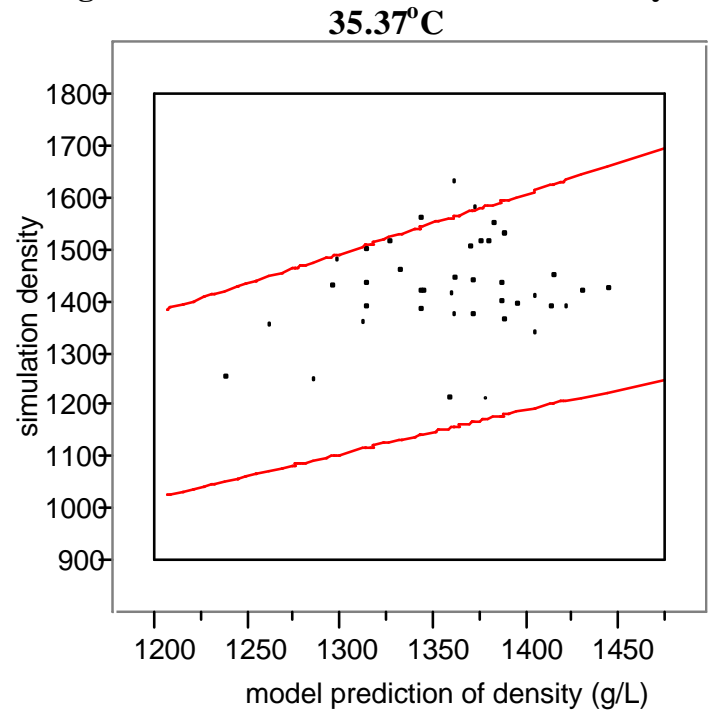

Plot of simulation vs. model heat capacity of the 41 OLH design points. The two lines indicate the $\pm 15 \%$ error bounds of the model given by Eq. (11).
Figure 4-12 Simulation vs. Model Water Mass 20-35.37 ${ }^{\circ} \mathrm{C}$

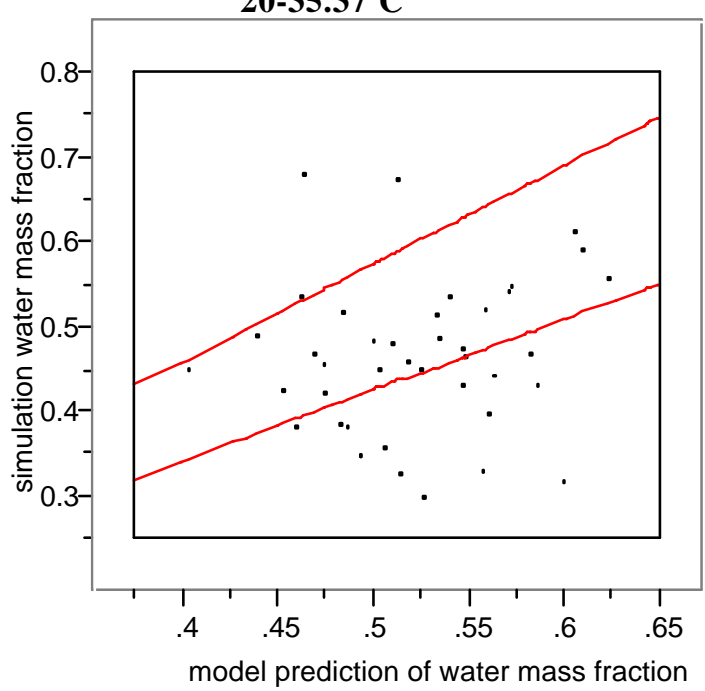

Plot of simulation vs. model water mass fraction of the 41 OLH design points. The two lines indicate the $\pm 15 \%$ error bounds of the model given by Eq. (10).

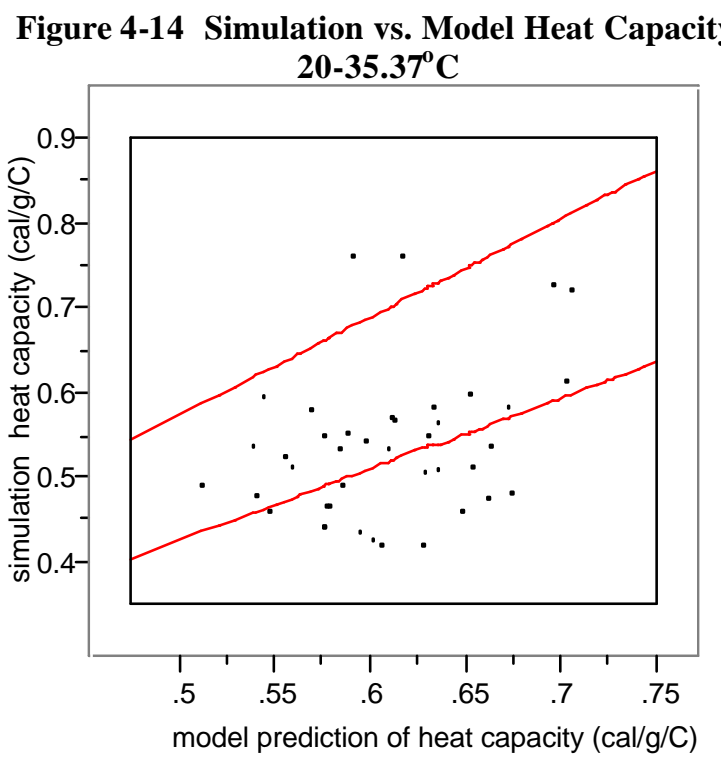

Plot of simulation vs. model density of the $41 \mathrm{OLH}$ design points. The two lines indicate the $\pm 15 \%$ error bounds of the model given by Eq. (12). 
Figure 4-15 Simulation vs. Linear Model for Viscosity $20-35.37^{\circ} \mathrm{C}$

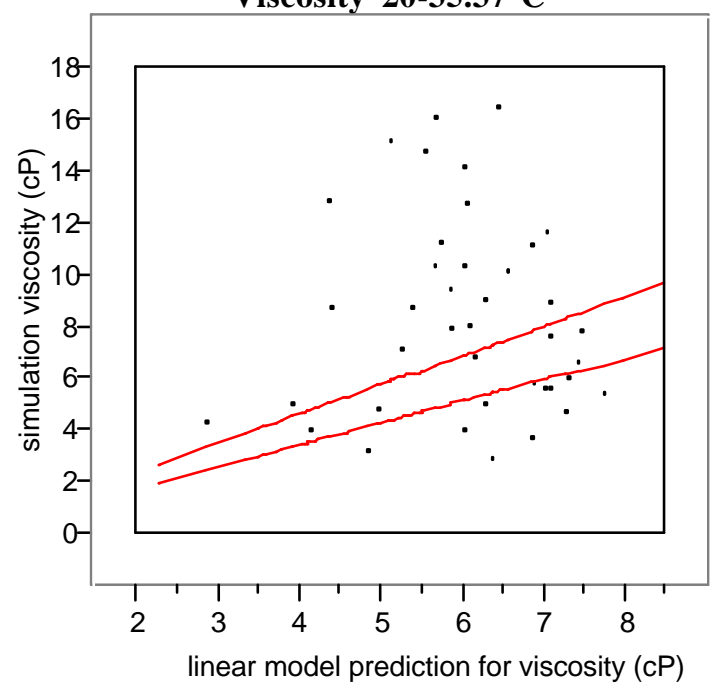

Plot of simulation vs. linear model for viscosity of the 41 OLH design points. The two lines indicate the $\pm 15 \%$ error bounds of the model given by Eq. (13).

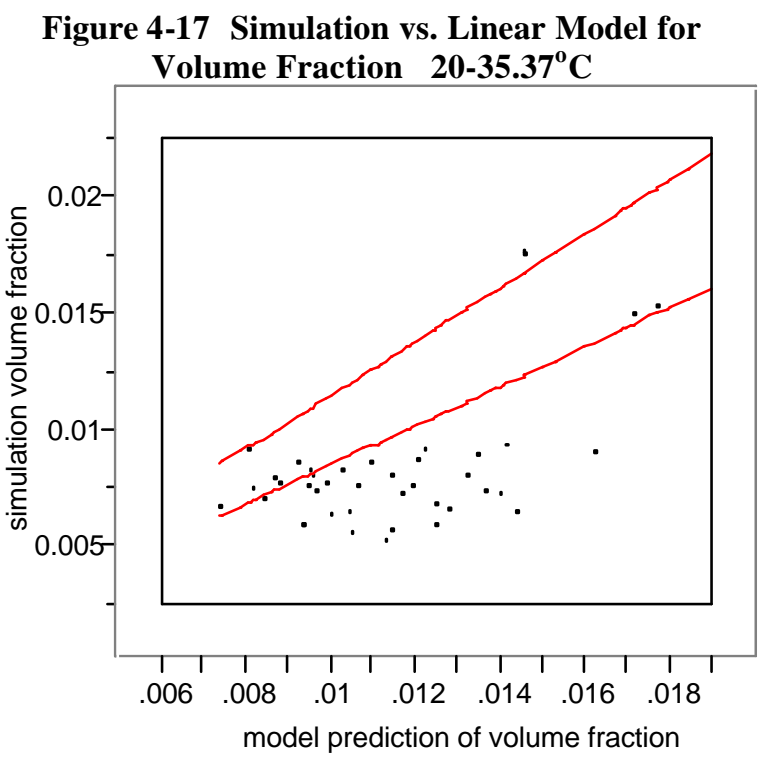

Plot of simulation vs. model volume fraction of the 41 OLH design points. The two lines indicate the $\pm 15 \%$ error bounds of the model given by Eq. (15)
Figure 4-16 Simulation vs. Nonlinear Model for Viscosity $20-35.37^{\circ} \mathrm{C}$

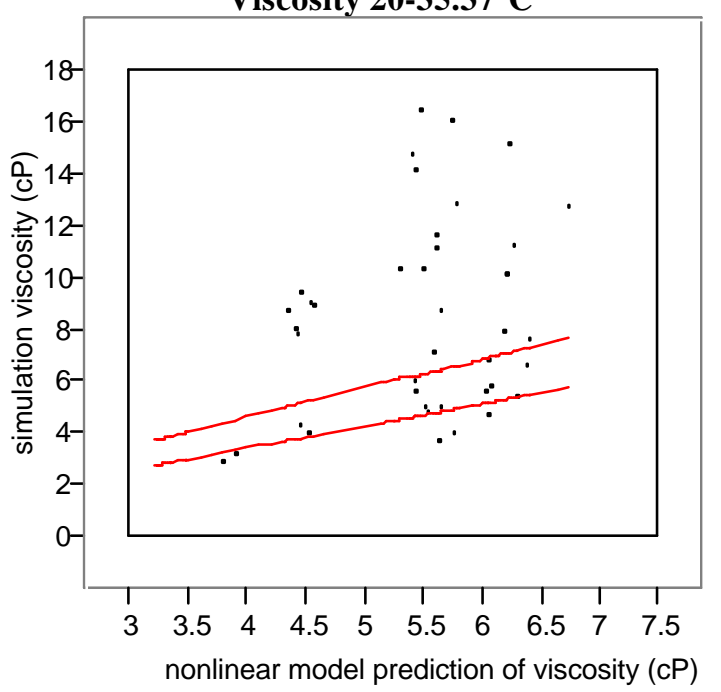

Plot of simulation vs. nonlinear model (Vogel) for viscosity of the 41 OLH design points. The two lines indicate the $\pm 15 \%$ error bounds of the model given by Eq. (14).

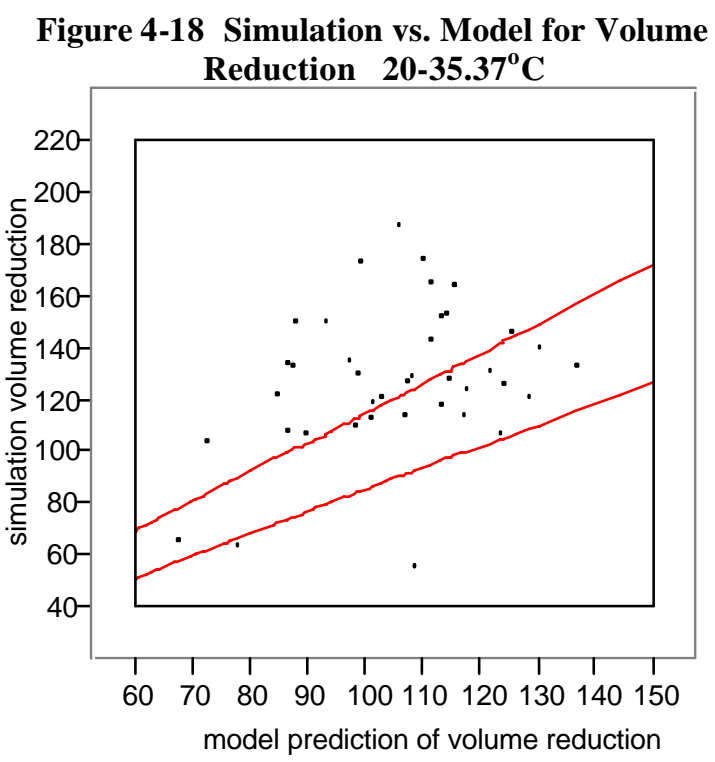

Plot of simulation vs. model volume reduction of the 41 OLH design points. The two lines indicate the $\pm 15 \%$ error bounds of the model given by Eq. (16). 
The expression for solubility is generally poorer than that for water mass fraction (WMF), and can be expressed in terms of WMF as:

$$
\text { Solubility }=(1-\mathrm{WMF}) / \mathrm{WMF}^{*} 1000
$$

In fact, this expression was used to calculate the solubility from the simulation results. It can be shown that the error in the mean of the percent difference for WMF will propagate to an error in the mean for solubility approximately in proportion to $1 / \mathrm{WMF}^{2}$. Because this is always greater than one, the error for solubility will always be greater than that for WMF. Also, it is expected that the fits of the two properties would perform differently because they are linear fits of properties having a non-linear relationship.

For this reason, and because the WMF is a more direct measurement than solubility when dealing with actual eluate samples in the lab, it is believed that water mass fraction (or equivalently, solids mass fraction) would provide a more accurate measure of the evaporation endpoint.

Table 4-3 and Table 4-4 list the simulation results (responses) for the 129 design points.

Table 4-3. Simulation Results (Responses) for $20-35.37^{\circ} \mathrm{C}$

\begin{tabular}{|c|c|c|c|c|c|c|c|}
\hline & temp $\left({ }^{\circ} \mathrm{C}\right)$ & \begin{tabular}{|l} 
water mass \\
fraction \\
(water mass/ \\
total mass)
\end{tabular} & $\begin{array}{l}\text { solubility } \\
\text { (mass solids/mass } \\
\text { water)*1000 }\end{array}$ & density $(\mathrm{g} / \mathrm{L})$ & $\mathrm{Cp}\left(\mathrm{cal} / \mathrm{g} /{ }^{\circ} \mathrm{C}\right)$ & $\begin{array}{l}\text { viscosity } \\
(\mathrm{cP})\end{array}$ & $\begin{array}{l}\text { volume fraction } \\
\text { (concentrated } \\
\text { vol/ feed vol) }\end{array}$ \\
\hline RTS001 & 20 & 0.80 & 245.21 & 1151.05 & 0.85 & 2.40 & 0.0293 \\
\hline RTS002 & 20 & 0.75 & 326.91 & 1186.14 & 0.82 & 2.86 & 0.0226 \\
\hline RTS003 & 20 & 0.71 & 409.05 & 1192.59 & 0.79 & 2.66 & 0.0199 \\
\hline RTS004 & 20 & 0.30 & 2379.74 & 1659.52 & 0.42 & 20.15 & 0.0053 \\
\hline RTS005 & 20 & 0.43 & 1317.55 & 1365.61 & 0.57 & 4.64 & 0.0089 \\
\hline RTS006 & 20 & 0.51 & 970.42 & 1391.64 & 0.59 & 6.74 & 0.0090 \\
\hline RTS007 & 20 & 0.44 & 1275.80 & 1435.96 & 0.53 & 6.00 & 0.0076 \\
\hline RTS008 & 20 & 0.43 & 1341.57 & 1414.25 & 0.59 & 7.41 & 0.0085 \\
\hline RTS009 & 35.369 & 0.57 & 769.34 & 1267.03 & 0.70 & 4.23 & 0.0149 \\
\hline RTS010 & 35.369 & 0.59 & 685.43 & 1361.74 & 0.63 & 6.53 & 0.0103 \\
\hline RTS011 & 35.369 & 0.45 & 1209.55 & 1402.12 & 0.52 & 7.77 & 0.0081 \\
\hline RTS012 & 35.369 & 0.57 & 747.61 & 1328.13 & 0.63 & 3.98 & 0.0101 \\
\hline RTS013 & 35.369 & 0.43 & 1340.00 & 1339.02 & 0.56 & 3.13 & 0.0089 \\
\hline RTS014 & 35.369 & 0.57 & 741.76 & 1314.08 & 0.60 & 3.27 & 0.0103 \\
\hline RTS015 & 35.369 & 0.39 & 1553.13 & 1437.95 & 0.51 & 4.73 & 0.0070 \\
\hline RTS016 & 35.369 & 0.56 & 783.71 & 1336.42 & 0.60 & 3.53 & 0.0098 \\
\hline RTS017 & 32.01 & 0.56 & 789.44 & 1363.98 & 0.62 & 5.06 & 0.0095 \\
\hline RTS018 & 22.88 & 0.68 & 478.01 & 1219.59 & 0.76 & 2.96 & 0.0176 \\
\hline RTS019 & 32.97 & 0.43 & 1307.77 & 1441.45 & 0.48 & 8.09 & 0.0074 \\
\hline RTS020 & 21.92 & 0.30 & 2327.98 & 1635.01 & 0.42 & 16.15 & 0.0053 \\
\hline RTS021 & 20.96 & 0.48 & 1095.83 & 1423.47 & 0.55 & 8.12 & 0.0082 \\
\hline RTS022 & 34.89 & 0.43 & 1344.59 & 1429.34 & 0.48 & 5.42 & 0.0071 \\
\hline RTS023 & 27.20 & 0.59 & 682.72 & 1264.80 & 0.72 & 4.32 & 0.0151 \\
\hline RTS024 & 28.65 & 0.62 & 625.21 & 1256.29 & 0.73 & 4.04 & 0.0155 \\
\hline RTS025 & 26.24 & 0.47 & 1126.74 & 1436.61 & 0.54 & 10.50 & 0.0081 \\
\hline RTS026 & 29.61 & 0.32 & 2142.12 & 1507.27 & 0.48 & 11.37 & 0.0066 \\
\hline RTS027 & 27.68 & 0.35 & 1853.04 & 1537.56 & 0.44 & 10.29 & 0.0060 \\
\hline RTS028 & 33.45 & 0.38 & 1621.63 & 1457.88 & 0.46 & 6.75 & 0.0068 \\
\hline RTS029 & 21.44 & 0.48 & 1072.82 & 1409.66 & 0.56 & 7.98 & 0.0084 \\
\hline RTS030 & 20.48 & 0.49 & 1039.01 & 1426.96 & 0.57 & 8.83 & 0.0083 \\
\hline RTS031 & 35.37 & 0.45 & 1211.20 & 1434.95 & 0.49 & 7.72 & 0.0074 \\
\hline RTS032 & 30.09 & 0.52 & 910.91 & 1380.59 & 0.57 & 6.08 & 0.0088 \\
\hline RTS033 & 24.80 & 0.39 & 1584.13 & 1521.04 & 0.47 & 11.79 & 0.0065 \\
\hline RTS034 & 31.05 & 0.55 & 811.59 & 1397.98 & 0.59 & 8.86 & 0.0091 \\
\hline RTS035 & 23.84 & 0.68 & 463.81 & 1224.53 & 0.76 & 3.25 & 0.0178 \\
\hline RTS036 & 32.01 & 0.54 & 836.48 & 1366.94 & 0.60 & 4.88 & 0.0092 \\
\hline RTS037 & 22.88 & 0.36 & 1782.46 & 1558.06 & 0.43 & 16.55 & 0.0060 \\
\hline RTS038 & 32.97 & 0.47 & 1139.64 & 1425.01 & 0.51 & 6.93 & 0.0076 \\
\hline RTS039 & 21.92 & 0.33 & 2035.86 & 1585.46 & 0.44 & 14.25 & 0.0057 \\
\hline RTS040 & 33.93 & 0.52 & 926.52 & 1371.39 & 0.58 & 4.08 & 0.0087 \\
\hline RTS041 & 20.96 & 0.45 & 1210.73 & 1449.19 & 0.53 & 9.12 & 0.0076 \\
\hline RTS042 & 34.89 & 0.47 & 1118.84 & 1398.81 & 0.53 & 4.78 & 0.0078 \\
\hline RTS042 & 27.20 & 0.44 & 1251.56 & 1488.20 & 0.51 & 12.94 & 0.0074 \\
\hline RTS044 & 28.65 & 0.40 & 1507.37 & 1521.30 & 0.46 & 15.22 & 0.0066 \\
\hline RTS045 & 26.24 & 0.43 & 1308.90 & 1467.12 & 0.51 & 10.40 & 0.0073 \\
\hline RTS046 & 29.61 & 0.33 & 2013.40 & 1567.01 & 0.42 & 12.81 & 0.0057 \\
\hline
\end{tabular}


WSRC-TR-2002-00319 Rev. 0 SRT-RPP-2002-00171

\begin{tabular}{|c|c|c|c|c|c|c|c|}
\hline & temp $\left({ }^{\circ} \mathrm{C}\right)$ & $\begin{array}{l}\text { water mass } \\
\text { fraction } \\
\text { (water mass/ } \\
\text { total mass) } \\
\end{array}$ & $\begin{array}{l}\text { solubility } \\
\text { (mass solids/mass } \\
\text { water)* }^{*} 1000\end{array}$ & density $(\mathrm{g} / \mathrm{L})$ & $\mathrm{Cp}\left(\mathrm{cal} / \mathrm{g} /{ }^{\circ} \mathrm{C}\right)$ & $\begin{array}{l}\text { viscosity } \\
(\mathrm{cP})\end{array}$ & $\begin{array}{l}\text { volume fraction } \\
\text { (concentrated } \\
\text { vol/ feed vol) }\end{array}$ \\
\hline RTS047 & 30.57 & 0.49 & 1052.00 & 1404.05 & 0.55 & 5.07 & 0.0080 \\
\hline RTS048 & 27.68 & 0.35 & 1853.82 & 1537.54 & 0.44 & 10.29 & 0.0060 \\
\hline RTS049 & 33.45 & 0.46 & 1181.52 & 1416.99 & 0.51 & 5.63 & 0.0075 \\
\hline RTS050 & 21.44 & 0.45 & 1203.48 & 1441.56 & 0.53 & 9.09 & 0.0077 \\
\hline RTS051 & 34.41 & 0.54 & 856.89 & 1347.15 & 0.60 & 3.70 & 0.0092 \\
\hline RTS052 & 20.48 & 0.46 & 1161.35 & 1451.47 & 0.55 & 9.56 & 0.0078 \\
\hline RTS053 & 35.37 & 0.49 & 1028.09 & 1396.78 & 0.54 & 5.84 & 0.0082 \\
\hline RTS051 & 34.41 & 0.54 & 856.89 & 1347.15 & 0.60 & 3.70 & 0.0092 \\
\hline RTS052 & 20.48 & 0.46 & 1161.35 & 1451.47 & 0.55 & 9.56 & 0.0078 \\
\hline RTS053 & 35.37 & 0.49 & 1028.09 & 1396.78 & 0.54 & 5.84 & 0.0082 \\
\hline RTS051 & 34.41 & 0.54 & 856.89 & 1347.15 & 0.60 & 3.70 & 0.0092 \\
\hline RTS052 & 20.48 & 0.46 & 1161.35 & 1451.47 & 0.55 & 9.56 & 0.0078 \\
\hline RTS053 & 35.37 & 0.49 & 1028.09 & 1396.78 & 0.54 & 5.84 & 0.0082 \\
\hline RTS054 & 30.09 & 0.52 & 927.29 & 1382.40 & 0.57 & 5.67 & 0.0087 \\
\hline RTS055 & 24.80 & 0.38 & 1612.64 & 1521.71 & 0.47 & 11.26 & 0.0065 \\
\hline RTS056 & 31.05 & 0.54 & 854.22 & 1390.08 & 0.59 & 7.21 & 0.0089 \\
\hline RTS057 & 23.84 & 0.42 & 1367.05 & 1514.83 & 0.49 & 14.84 & 0.0069 \\
\hline RTS058 & 20 & 0.69 & $\begin{array}{r}449.99 \\
\end{array}$ & 1207.55 & 0.78 & 2.75 & 0.0183 \\
\hline RTS059 & 20 & 0.77 & 303.18 & 1170.07 & 0.83 & 2.46 & 0.0245 \\
\hline RTS060 & 20 & 0.76 & 315.49 & 1172.79 & 0.83 & 2.53 & 0.0238 \\
\hline RTS061 & 20 & 0.72 & 380.99 & 1189.82 & 0.80 & 2.67 & 0.0207 \\
\hline RTS062 & 20 & 0.73 & 375.75 & 1188.66 & 0.81 & 2.68 & 0.0209 \\
\hline RTS063 & 20 & 0.79 & 263.90 & 1158.03 & 0.85 & 2.46 & 0.0274 \\
\hline RTS064 & 20 & 0.40 & 1515.70 & 1444.66 & 0.56 & 11.66 & 0.0080 \\
\hline RTS065 & 20 & 0.68 & 465.61 & 1217.90 & 0.77 & 3.19 & 0.0178 \\
\hline RTS066 & 20 & 0.77 & 296.39 & 1167.64 & 0.83 & 2.49 & 0.0249 \\
\hline RTS067 & 20 & 0.65 & 528.31 & 1225.90 & 0.76 & 3.25 & 0.0168 \\
\hline RTS068 & 20 & 0.75 & 340.91 & 1186.87 & 0.81 & 2.86 & 0.0221 \\
\hline RTS069 & 20 & 0.80 & 249.09 & 1153.20 & 0.85 & 2.42 & 0.0289 \\
\hline RTS070 & 20 & 0.73 & 361.93 & 1184.68 & 0.81 & 2.52 & 0.0213 \\
\hline RTS071 & 20 & 0.53 & 892.10 & 1267.66 & 0.71 & 3.68 & 0.0135 \\
\hline RTS072 & 20 & 0.79 & 268.14 & 1158.31 & 0.85 & 2.43 & 0.0272 \\
\hline RTS073 & 20 & 0.53 & 870.19 & 1288.83 & 0.69 & 4.32 & 0.0125 \\
\hline RTS074 & 20 & 0.74 & 352.72 & 1183.62 & 0.82 & 2.55 & 0.0216 \\
\hline RTS075 & 20 & 0.75 & 327.24 & 1174.00 & 0.82 & 2.51 & 0.0234 \\
\hline RTS076 & 20 & 0.40 & 1472.88 & 1442.05 & 0.57 & 11.22 & 0.0081 \\
\hline RTS077 & 20 & 0.75 & 338.74 & 1179.35 & 0.82 & 2.65 & 0.0226 \\
\hline RTS078 & 20 & 0.77 & 300.13 & 1167.76 & 0.84 & 2.51 & 0.0249 \\
\hline RTS079 & 20 & 0.76 & 315.11 & 1172.81 & 0.83 & 2.56 & 0.0239 \\
\hline RTS080 & 20 & 0.67 & 491.57 & 1225.46 & 0.76 & 3.03 & 0.0170 \\
\hline RTS081 & 20 & 0.62 & 623.87 & 1234.86 & 0.74 & 3.26 & 0.0158 \\
\hline RTS082 & 20 & 0.64 & 574.31 & 1231.63 & 0.75 & 3.23 & 0.0161 \\
\hline RTS083 & 20 & 0.80 & 252.92 & 1153.09 & 0.85 & 2.43 & 0.0286 \\
\hline RTS084 & 20 & 0.78 & 276.85 & 1161.47 & 0.85 & 2.48 & 0.0266 \\
\hline RTS085 & 20 & 0.76 & 316.13 & 1173.07 & 0.83 & 2.53 & 0.0238 \\
\hline RTS086 & 20 & 0.65 & 549.79 & 1229.07 & 0.77 & 2.97 & 0.0162 \\
\hline RTS087 & 20 & 0.78 & 285.46 & 1163.91 & 0.83 & 2.41 & 0.0257 \\
\hline RTS088 & 20 & 0.78 & 275.88 & 1161.85 & 0.84 & 2.47 & 0.0265 \\
\hline RTS089 & 20 & 0.78 & 278.93 & 1162.27 & 0.85 & 2.46 & 0.0263 \\
\hline RTS090 & 20 & 0.40 & 1514.95 & 1514.55 & 0.48 & 15.03 & 0.0067 \\
\hline RTS091 & 20 & 0.79 & 264.09 & 1158.45 & 0.85 & 2.43 & 0.0274 \\
\hline RTS092 & 20 & 0.70 & 427.07 & 1200.28 & 0.78 & 2.78 & 0.0191 \\
\hline RTS093 & 20 & 0.73 & 360.74 & 1187.34 & 0.81 & 2.70 & 0.0214 \\
\hline RTS094 & 35.369 & 0.40 & 1497.68 & 1400.41 & 0.54 & 6.97 & 0.0083 \\
\hline RTS095 & 35.369 & 0.47 & 1127.61 & 1377.83 & 0.57 & 7.56 & 0.0095 \\
\hline RTS096 & 35.369 & 0.47 & 1116.20 & 1403.01 & 0.55 & 8.81 & 0.0087 \\
\hline RTS097 & 35.369 & 0.49 & 1048.21 & 1416.03 & 0.53 & 8.40 & 0.0081 \\
\hline RTS098 & 35.369 & 0.51 & 971.57 & 1401.71 & 0.52 & 7.38 & 0.0084 \\
\hline RTS099 & 35.369 & 0.53 & 893.24 & 1282.64 & 0.68 & 4.40 & 0.0136 \\
\hline RTS100 & 35.369 & 0.59 & 695.12 & 1314.71 & 0.64 & 3.99 & 0.0106 \\
\hline RTS101 & 35.369 & 0.59 & 697.70 & 1334.01 & 0.64 & 4.78 & 0.0105 \\
\hline RTS102 & 35.369 & 0.47 & 1115.14 & 1373.90 & 0.57 & 7.86 & 0.0095 \\
\hline RTS103 & 35.369 & 0.58 & 736.08 & 1335.77 & 0.62 & 4.70 & 0.0102 \\
\hline RTS104 & 35.369 & 0.59 & 695.75 & 1355.80 & 0.63 & 6.25 & 0.0102 \\
\hline RTS105 & 35.369 & 0.56 & 773.36 & 1269.42 & 0.70 & 4.29 & 0.0146 \\
\hline RTS106 & 35.369 & 0.45 & 1202.31 & 1416.41 & 0.51 & 8.60 & 0.0080 \\
\hline RTS107 & 35.369 & 0.51 & 970.14 & 1386.12 & 0.56 & 5.46 & 0.0085 \\
\hline RTS108 & 35.369 & 0.51 & 963.22 & 1287.83 & 0.67 & 4.45 & 0.0134 \\
\hline RTS109 & 35.369 & 0.56 & 790.63 & 1341.37 & 0.61 & 4.29 & 0.0097 \\
\hline RTS110 & 35.369 & 0.39 & 1572.93 & 1423.40 & 0.50 & 8.27 & 0.0079 \\
\hline RTS111 & 35.369 & 0.46 & 1155.94 & 1431.86 & 0.51 & 9.91 & 0.0080 \\
\hline RTS112 & 35.369 & 0.57 & 741.70 & 1332.48 & 0.63 & 4.23 & 0.0101 \\
\hline RTS113 & 35.369 & 0.54 & 861.15 & 1384.46 & 0.57 & 7.90 & 0.0091 \\
\hline RTS114 & 35.369 & 0.48 & 1096.22 & 1377.96 & 0.57 & 7.89 & 0.0096 \\
\hline RTS115 & 35.369 & 0.51 & 977.77 & 1410.55 & 0.55 & 9.27 & 0.0085 \\
\hline
\end{tabular}


WSRC-TR-2002-00319 Rev. 0 SRT-RPP-2002-00171

\begin{tabular}{|c|c|c|c|c|c|c|c|}
\hline & temp $\left({ }^{\circ} \mathrm{C}\right)$ & $\begin{array}{l}\text { water mass } \\
\text { fraction } \\
\text { (water mass/ } \\
\text { total mass) }\end{array}$ & $\begin{array}{l}\begin{array}{l}\text { solubility } \\
\text { (mass solids/mass } \\
\text { water)* } 1000\end{array} \\
\end{array}$ & density $(\mathrm{g} / \mathrm{L})$ & $\mathrm{Cp}\left(\mathrm{cal} / \mathrm{g} /{ }^{\circ} \mathrm{C}\right)$ & $\begin{array}{l}\text { viscosity } \\
(\mathrm{cP})\end{array}$ & $\begin{array}{l}\text { volume fraction } \\
\text { (concentrated } \\
\text { vol/ feed vol) }\end{array}$ \\
\hline RTS116 & 35.369 & 0.53 & 893.56 & 1389.79 & 0.57 & 5.86 & 0.0087 \\
\hline RTS117 & 35.369 & 0.54 & 852.24 & 1361.87 & 0.63 & 5.21 & 0.0092 \\
\hline RTS 118 & 35.369 & 0.55 & 830.68 & 1359.06 & 0.59 & 5.16 & 0.0094 \\
\hline RTS119 & 35.369 & 0.55 & 824.64 & 1275.21 & 0.70 & 4.40 & 0.0143 \\
\hline RTS120 & 35.369 & 0.49 & 1042.61 & 1302.13 & 0.66 & 4.93 & 0.0126 \\
\hline RTS121 & 35.369 & 0.47 & 1119.19 & 1403.20 & 0.54 & 8.75 & 0.0087 \\
\hline RTS122 & 35.369 & 0.45 & 1221.12 & 1393.93 & 0.54 & 5.84 & 0.0083 \\
\hline RTS123 & 35.369 & 0.47 & 1108.66 & 1314.36 & 0.64 & 5.00 & 0.0119 \\
\hline RTS124 & 35.369 & 0.49 & 1038.14 & 1300.58 & 0.66 & 4.89 & 0.0126 \\
\hline RTS125 & 35.369 & 0.49 & 1051.27 & 1303.23 & 0.66 & 4.88 & 0.0124 \\
\hline RTS126 & 35.369 & 0.40 & 1508.92 & 1467.28 & 0.44 & 8.66 & 0.0067 \\
\hline RTS127 & 35.369 & 0.53 & 880.43 & 1282.76 & 0.68 & 4.36 & 0.0137 \\
\hline RTS128 & 35.369 & 0.48 & 1061.95 & 1408.49 & 0.53 & 7.75 & 0.0081 \\
\hline RTS129 & 35.369 & 0.54 & 868.20 & 1387.82 & 0.57 & 7.51 & 0.0090 \\
\hline
\end{tabular}

Table 4-4. Simulation Results (Responses) for $35.37-70^{\circ} \mathrm{C}$

\begin{tabular}{|c|c|c|c|c|c|c|c|}
\hline Run & temp $\left({ }^{\circ} \mathrm{C}\right)$ & \begin{tabular}{|l} 
water mass \\
fraction \\
(water mass/ \\
total mass)
\end{tabular} & $\begin{array}{l}\begin{array}{l}\text { solubility } \\
\text { (mass solids/mass } \\
\text { water)* } 1000\end{array} \\
\end{array}$ & density (g/L) & $\mathrm{Cp}\left(\mathrm{cal} / \mathrm{g} /{ }^{\circ} \mathrm{C}\right)$ & $\begin{array}{l}\text { viscosity } \\
(\mathrm{cP})\end{array}$ & $\begin{array}{l}\text { volume fraction } \\
\text { (concentrated } \\
\text { vol/ feed vol) }\end{array}$ \\
\hline RTS001 & 35.37 & 0.64 & 566.60 & 1295.97 & 0.67 & 5.29 & 0.0133 \\
\hline RTS002 & 35.37 & 0.59 & 685.42 & 1359.43 & 0.62 & 6.42 & 0.0104 \\
\hline RTS003 & 35.37 & 0.59 & 704.41 & 1299.06 & 0.64 & 3.97 & 0.0115 \\
\hline \begin{tabular}{|l|} 
RTS004 \\
\end{tabular} & 35.37 & 0.57 & 747.60 & 1328.12 & 0.63 & 3.98 & 0.0101 \\
\hline \begin{tabular}{|l|} 
RTS005 \\
\end{tabular} & 35.37 & 0.46 & 1186.88 & 1339.02 & 0.56 & 3.13 & 0.0089 \\
\hline RTS006 & 35.37 & 0.57 & 741.76 & 1314.08 & 0.60 & 3.27 & 0.0103 \\
\hline RTS007 & 35.37 & 0.43 & 1337.25 & 1411.05 & 0.50 & 4.05 & 0.0076 \\
\hline RTS008 & 35.37 & 0.56 & 783.71 & 1336.42 & 0.60 & 3.53 & 0.0098 \\
\hline $\begin{array}{l}\text { RTS009 } \\
\end{array}$ & 70 & 0.66 & 507.47 & 1228.68 & 0.68 & 1.85 & 0.0151 \\
\hline RTS010 & 70 & 0.65 & 538.02 & 1253.26 & 0.69 & 1.75 & 0.0129 \\
\hline RTS011 & 70 & 0.61 & 642.68 & 1235.93 & 0.65 & 1.67 & 0.0128 \\
\hline RTS012 & 70 & 0.63 & 598.50 & 1233.45 & 0.69 & 1.51 & 0.0124 \\
\hline RTS013 & 70 & 0.49 & 1039.34 & 1290.57 & 0.56 & 1.64 & 0.0092 \\
\hline \begin{tabular}{|l} 
RTS014 \\
\end{tabular} & 70 & 0.62 & 613.53 & 1228.77 & 0.66 & 1.32 & 0.0124 \\
\hline RTS015 & 70 & 0.46 & 1163.55 & 1325.22 & 0.54 & 1.74 & 0.0086 \\
\hline RTS016 & 70 & 0.60 & 654.42 & 1250.43 & 0.65 & 1.40 & 0.0116 \\
\hline \begin{tabular}{|l} 
RTS017 \\
\end{tabular} & 62.42 & 0.61 & 650.11 & 1269.32 & 0.66 & 1.87 & 0.0114 \\
\hline \begin{tabular}{|l|} 
RTS018 \\
\end{tabular} & 41.86 & 0.58 & 713.08 & 1299.92 & 0.62 & 3.50 & 0.0114 \\
\hline RTS019 & 64.59 & 0.58 & 737.57 & 1265.65 & 0.62 & 1.89 & 0.0115 \\
\hline \begin{tabular}{|l} 
RTS020 \\
\end{tabular} & 39.70 & 0.56 & 792.44 & 1331.94 & 0.62 & 3.35 & 0.0097 \\
\hline RTS021 & 37.53 & 0.51 & 947.71 & 1349.55 & 0.57 & 3.85 & 0.0093 \\
\hline $\begin{array}{l}\text { RTS022 } \\
\end{array}$ & 68.92 & 0.49 & 1055.77 & 1304.73 & 0.56 & 1.84 & 0.0092 \\
\hline \begin{tabular}{|l} 
RTS023 \\
\end{tabular} & 51.60 & 0.61 & 642.19 & 1290.89 & 0.64 & 3.15 & 0.0122 \\
\hline RTS024 & 54.85 & 0.63 & 594.09 & 1265.72 & 0.66 & 2.69 & 0.0132 \\
\hline RTS025 & 49.44 & 0.58 & 733.40 & 1287.18 & 0.62 & 2.85 & 0.0115 \\
\hline $\begin{array}{l}\text { RTS026 } \\
\end{array}$ & 57.01 & 0.60 & 670.34 & 1274.09 & 0.65 & 2.24 & 0.0113 \\
\hline RTS027 & 52.69 & 0.54 & 860.81 & 1317.89 & 0.59 & 2.48 & 0.0094 \\
\hline $\begin{array}{l}\text { RTS028 } \\
\end{array}$ & 65.67 & 0.51 & 956.82 & 1297.12 & 0.58 & 1.81 & 0.0097 \\
\hline \begin{tabular}{|l|} 
RTS029 \\
\end{tabular} & 38.62 & 0.53 & 890.31 & 1328.83 & 0.59 & 3.55 & 0.0098 \\
\hline RTS030 & 36.45 & 0.56 & 778.98 & 1339.03 & 0.62 & 3.81 & 0.0097 \\
\hline RTS031 & 70.00 & 0.57 & 748.48 & 1263.72 & 0.62 & 1.73 & 0.0115 \\
\hline \begin{tabular}{|l|} 
RTS032 \\
\end{tabular} & 58.10 & 0.56 & 788.58 & 1286.03 & 0.60 & 2.27 & 0.0105 \\
\hline RTS033 & 46.19 & 0.56 & 780.28 & 1311.53 & 0.60 & 2.95 & 0.0100 \\
\hline RTS034 & 60.26 & 0.60 & 669.99 & 1277.81 & 0.63 & 2.38 & 0.0120 \\
\hline RTS035 & 44.03 & 0.59 & 690.38 & 1305.81 & 0.63 & 3.75 & 0.0116 \\
\hline $\begin{array}{l}\text { RTS036 } \\
\end{array}$ & 62.42 & 0.59 & 697.39 & 1275.47 & 0.64 & 1.87 & 0.0109 \\
\hline RTS037 & 41.86 & 0.55 & 808.32 & 1317.17 & 0.60 & 3.49 & 0.0105 \\
\hline $\begin{array}{l}\text { RTS038 } \\
\end{array}$ & 64.59 & 0.55 & 821.48 & 1281.28 & 0.60 & 1.95 & 0.0107 \\
\hline RTS039 & 39.70 & 0.55 & 827.50 & 1337.82 & 0.61 & 3.41 & 0.0095 \\
\hline RTS040 & 66.75 & 0.56 & 770.17 & 1278.87 & 0.63 & 1.63 & 0.0103 \\
\hline \begin{tabular}{|l|} 
RTS041 \\
\end{tabular} & 37.53 & 0.50 & 998.48 & 1359.52 & 0.56 & 4.00 & 0.0090 \\
\hline $\begin{array}{l}\text { RTS042 } \\
\end{array}$ & 68.92 & 0.51 & 954.27 & 1304.74 & 0.57 & 1.87 & 0.0092 \\
\hline RTS042 & 51.60 & 0.58 & 711.63 & 1301.81 & 0.62 & 2.98 & 0.0111 \\
\hline \begin{tabular}{|l|} 
RTS044 \\
\end{tabular} & 54.85 & 0.59 & 694.78 & 1280.62 & 0.63 & 2.58 & 0.0118 \\
\hline \begin{tabular}{|l|l} 
RTS045 \\
\end{tabular} & 49.44 & 0.55 & 820.72 & 1304.88 & 0.60 & 2.86 & 0.0105 \\
\hline RTS046 & 57.01 & 0.58 & 725.88 & 1285.07 & 0.63 & 2.22 & 0.0107 \\
\hline RTS047 & 59.18 & 0.52 & 918.62 & 1320.01 & 0.58 & 2.14 & 0.0091 \\
\hline \begin{tabular}{|l|} 
RTS048 \\
\end{tabular} & 52.69 & 0.54 & 860.80 & 1317.89 & 0.59 & 2.48 & 0.0094 \\
\hline \begin{tabular}{|l|} 
RTS049 \\
\end{tabular} & 65.67 & 0.51 & 979.14 & 1304.07 & 0.58 & 1.93 & 0.0095 \\
\hline
\end{tabular}


WSRC-TR-2002-00319 Rev. 0 SRT-RPP-2002-00171

\begin{tabular}{|c|c|c|c|c|c|c|c|}
\hline Run & temp $\left({ }^{\circ} \mathrm{C}\right)$ & $\begin{array}{l}\text { water mass } \\
\text { fraction } \\
\text { (water mass/ } \\
\text { total mass) }\end{array}$ & $\begin{array}{l}\text { solubility } \\
\text { (mass solids/mass } \\
\text { water)*1000 }\end{array}$ & density (g/L) & $\mathrm{Cp}\left(\mathrm{cal} / \mathrm{g} /{ }^{\circ} \mathrm{C}\right)$ & $\begin{array}{l}\text { viscosity } \\
(\mathrm{cP})\end{array}$ & $\begin{array}{l}\text { volume fraction } \\
\text { (concentrated } \\
\text { vol/ feed vol) }\end{array}$ \\
\hline RTS050 & 38.62 & 0.51 & 959.72 & 1345.31 & 0.57 & 3.75 & 0.0093 \\
\hline RTS051 & 67.84 & 0.58 & 721.16 & 1261.69 & 0.65 & 1.52 & 0.0108 \\
\hline RTS052 & 36.45 & 0.55 & 829.57 & 1347.76 & 0.60 & 3.87 & 0.0094 \\
\hline RTS053 & 70.00 & 0.55 & 826.39 & 1278.22 & 0.61 & 1.79 & 0.0106 \\
\hline RTS051 & 58.10 & 0.55 & 802.91 & 1297.92 & 0.60 & 2.29 & 0.0100 \\
\hline RTS052 & 46.19 & 0.55 & 820.35 & 1320.58 & 0.60 & 2.96 & 0.0097 \\
\hline RTS053 & 60.26 & 0.58 & 726.45 & 1288.39 & 0.62 & 2.31 & 0.0110 \\
\hline RTS051 & 44.03 & 0.56 & 784.42 & 1318.6 & 0.61 & 3.56 & 0.0107 \\
\hline RTS052 & 62.42 & 0.56 & 778.48 & 1324.58 & 0.62 & 4.17 & 0.0105 \\
\hline RTS053 & 41.86 & 0.60 & 671.84 & 1315.94 & 0.64 & 4.85 & 0.0116 \\
\hline RTS054 & 64.59 & 0.60 & 660.11 & 1307.88 & 0.65 & 4.68 & 0.0118 \\
\hline RTS055 & 39.70 & 0.58 & 712.85 & 1311.96 & 0.64 & 4.38 & 0.0112 \\
\hline RTS056 & 37.53 & 0.57 & 764.00 & 1327.36 & 0.61 & 4.77 & 0.0109 \\
\hline RTS057 & 68.92 & 0.62 & 620.14 & 1308.2 & 0.65 & 5.31 & 0.0123 \\
\hline RTS058 & 35.37 & 0.59 & 695.12 & 1314.7 & 0.64 & 3.99 & 0.0106 \\
\hline RTS059 & 35.37 & 0.59 & 697.70 & 1334 & 0.64 & 4.78 & 0.0105 \\
\hline RTS060 & 35.37 & 0.61 & 648.03 & 1308.05 & 0.64 & 4.93 & 0.0119 \\
\hline RTS061 & 35.37 & 0.58 & 736.08 & 1335.76 & 0.62 & 4.70 & 0.0102 \\
\hline RTS062 & 35.37 & 0.59 & 695.73 & 1353.56 & 0.63 & 6.10 & 0.0105 \\
\hline RTS063 & 35.37 & 0.64 & 566.86 & 1296.49 & 0.67 & 5.29 & 0.0131 \\
\hline RTS064 & 35.37 & 0.59 & 690.52 & 1307.21 & 0.64 & 4.21 & 0.0114 \\
\hline RTS065 & 35.37 & 0.53 & 900.34 & 1348.39 & 0.59 & 4.32 & 0.0095 \\
\hline RTS066 & 35.37 & 0.61 & 628.55 & 1309.1 & 0.65 & 5.19 & 0.0124 \\
\hline RTS067 & 35.37 & 0.56 & 790.63 & 1341.36 & 0.61 & 4.29 & 0.0097 \\
\hline RTS068 & 35.37 & 0.57 & 740.45 & 1323.22 & 0.62 & 4.60 & 0.0109 \\
\hline RTS069 & 35.37 & 0.60 & 667.20 & 1305.65 & 0.65 & 4.49 & 0.0120 \\
\hline RTS070 & 35.37 & 0.57 & 741.69 & 1332.47 & 0.63 & 4.23 & 0.0101 \\
\hline RTS071 & 35.37 & 0.59 & 681.19 & 1310.78 & 0.64 & 4.78 & 0.0116 \\
\hline RTS072 & 35.37 & 0.60 & 656.39 & 1310.53 & 0.65 & 4.92 & 0.0120 \\
\hline RTS073 & 35.37 & 0.60 & 656.90 & 1307.67 & 0.65 & 4.78 & 0.0119 \\
\hline RTS074 & 35.37 & 0.53 & 893.55 & 1372.53 & 0.58 & 5.25 & 0.0093 \\
\hline RTS075 & 35.37 & 0.54 & 836.39 & 1336.85 & 0.61 & 4.45 & 0.0100 \\
\hline RTS076 & 35.37 & 0.55 & 830.68 & 1339.12 & 0.61 & 4.55 & 0.0100 \\
\hline RTS077 & 35.37 & 0.63 & 575.10 & 1296.65 & 0.67 & 5.18 & 0.0132 \\
\hline RTS078 & 35.37 & 0.62 & 621.87 & 1306.35 & 0.66 & 5.08 & 0.0124 \\
\hline RTS079 & 35.37 & 0.60 & 663.82 & 1308.99 & 0.65 & 4.69 & 0.0118 \\
\hline RTS080 & 35.37 & 0.53 & 881.10 & 1345.63 & 0.59 & 4.30 & 0.0097 \\
\hline RTS081 & 35.37 & 0.61 & 652.13 & 1312.79 & 0.64 & 4.94 & 0.0120 \\
\hline RTS082 & 35.37 & 0.62 & 620.02 & 1306.28 & 0.66 & 5.09 & 0.0123 \\
\hline RTS083 & 35.37 & 0.61 & 632.04 & 1307.58 & 0.65 & 5.03 & 0.0122 \\
\hline RTS084 & 35.37 & 0.54 & 846.68 & 1333.45 & 0.60 & 4.06 & 0.0101 \\
\hline RTS085 & 35.37 & 0.62 & 613.09 & 1308.27 & 0.65 & 5.26 & 0.0124 \\
\hline RTS086 & 35.37 & 0.57 & 744.36 & 1314.6 & 0.63 & 4.28 & 0.0109 \\
\hline RTS087 & 35.37 & 0.58 & 714.39 & 1321.37 & 0.64 & 4.78 & 0.0111 \\
\hline RTS088 & 35.37 & 0.59 & 706.72 & 1257.76 & 0.63 & 1.70 & 0.0118 \\
\hline RTS089 & 35.37 & 0.63 & 595.49 & 1243.71 & 0.66 & 1.78 & 0.0134 \\
\hline RTS090 & 35.37 & 0.63 & 598.60 & 1241.71 & 0.66 & 1.79 & 0.0133 \\
\hline RTS091 & 35.37 & 0.61 & 652.71 & 1247.18 & 0.65 & 1.76 & 0.0126 \\
\hline RTS092 & 35.37 & 0.59 & 685.67 & 1254.3 & 0.64 & 1.84 & 0.0123 \\
\hline RTS093 & 35.37 & 0.65 & 550.38 & 1236.69 & 0.67 & 1.85 & 0.0142 \\
\hline RTS094 & 70 & 0.63 & 574.84 & 1229.64 & 0.68 & 1.50 & 0.0128 \\
\hline RTS095 & 70 & 0.64 & 574.78 & 1244.87 & 0.68 & 1.65 & 0.0126 \\
\hline RTS096 & 70 & 0.63 & 579.42 & 1237.86 & 0.66 & 1.81 & 0.0136 \\
\hline RTS097 & 70 & 0.62 & 614.36 & 1248.67 & 0.67 & 1.69 & 0.0122 \\
\hline RTS098 & 70 & 0.65 & 543.26 & 1247.76 & 0.68 & 1.75 & 0.0130 \\
\hline RTS099 & 70 & 0.66 & 508.86 & 1229.94 & 0.68 & 1.85 & 0.0149 \\
\hline RTS100 & 70 & 0.62 & 625.09 & 1241.29 & 0.65 & 1.68 & 0.0129 \\
\hline RTS101 & 70 & 0.55 & 828.30 & 1280.69 & 0.61 & 1.80 & 0.0105 \\
\hline RTS102 & 70 & 0.64 & 554.60 & 1236.2 & 0.67 & 1.82 & 0.0142 \\
\hline RTS103 & 70 & 0.60 & 664.99 & 1255.82 & 0.65 & 1.62 & 0.0115 \\
\hline RTS104 & 70 & 0.60 & 656.69 & 1250.29 & 0.64 & 1.77 & 0.0125 \\
\hline RTS105 & 70 & 0.62 & 606.37 & 1240.17 & 0.66 & 1.76 & 0.0134 \\
\hline RTS106 & 70 & 0.62 & 617.54 & 1246.11 & 0.67 & 1.57 & 0.0121 \\
\hline RTS107 & 70 & 0.62 & 625.02 & 1245.68 & 0.65 & 1.84 & 0.0130 \\
\hline RTS108 & 70 & 0.63 & 590.86 & 1241.7 & 0.66 & 1.83 & 0.0136 \\
\hline RTS109 & 70 & 0.63 & 597.78 & 1242.23 & 0.66 & 1.82 & 0.0133 \\
\hline RTS110 & 70 & 0.59 & 708.47 & 1280.43 & 0.63 & 1.84 & 0.0107 \\
\hline RTS111 & 70 & 0.58 & 725.21 & 1270.51 & 0.63 & 1.83 & 0.0111 \\
\hline RTS112 & 70 & 0.59 & 703.29 & 1270.76 & 0.63 & 1.84 & 0.0110 \\
\hline RTS113 & 70 & 0.66 & 517.64 & 1230.46 & 0.68 & 1.85 & 0.0149 \\
\hline RTS114 & 70 & 0.64 & 557.01 & 1238.1 & 0.67 & 1.84 & 0.0141 \\
\hline RTS115 & 70 & 0.62 & 601.29 & 1242.25 & 0.66 & 1.79 & 0.0133 \\
\hline RTS116 & 70 & 0.56 & 788.36 & 1271.65 & 0.61 & 1.76 & 0.0109 \\
\hline RTS117 & 70 & 0.64 & 574.02 & 1239.13 & 0.66 & 1.78 & 0.0138 \\
\hline RTS118 & 70 & 0.64 & 555.69 & 1237.79 & 0.67 & 1.83 & 0.0140 \\
\hline
\end{tabular}


WSRC-TR-2002-00319 Rev. 0

SRT-RPP-2002-00171

\begin{tabular}{|l|r|l|l|r|r|r|r|}
\hline Run & temp ${ }^{\circ} \mathrm{C}$ ) & $\begin{array}{l}\text { Water mass } \\
\text { fraction } \\
\text { (water mass/ } \\
\text { total mass) }\end{array}$ & $\begin{array}{l}\text { lolubility } \\
\text { (mass solids/mass } \\
\text { water)*1000 }\end{array}$ & density (g/L) & Cp (cal/g/ $\left.{ }^{\circ} \mathrm{C}\right)$ & $\begin{array}{l}\text { viscosity } \\
(\mathrm{cP})\end{array}$ & $\begin{array}{l}\text { volume fraction } \\
(\mathrm{concentrated} \\
\text { vol/ feed vol) }\end{array}$ \\
\hline RTS119 & 70 & 0.64 & 564.38 & 1237.78 & 0.67 & 1.82 & 0.0139 \\
\hline RTS120 & 70 & 0.57 & 768.23 & 1264.29 & 0.62 & 1.68 & 0.0113 \\
\hline RTS121 & 70 & 0.65 & 543.93 & 1236.69 & 0.67 & 1.83 & 0.0143 \\
\hline RTS122 & 70 & 0.59 & 681.61 & 1249.48 & 0.64 & 1.75 & 0.0122 \\
\hline RTS123 & 70 & 0.60 & 655.33 & 1255.39 & 0.65 & 1.84 & 0.0124 \\
\hline RTS124 & 70 & 0.64 & 566.60 & 1295.97 & 0.67 & 5.29 & 0.0133 \\
\hline RTS125 & 70 & 0.59 & 685.42 & 1359.43 & 0.62 & 6.42 & 0.0104 \\
\hline RTS126 & 70 & 0.59 & 704.41 & 1299.06 & 0.64 & 3.97 & 0.0115 \\
\hline RTS127 & 70 & 0.57 & 747.60 & 1328.12 & 0.63 & 3.98 & 0.0101 \\
\hline RTS128 & 70 & 0.46 & 1186.88 & 1339.02 & 0.56 & 3.13 & 0.0089 \\
\hline RTS129 & 70 & 0.57 & 741.76 & 1314.08 & 0.60 & 3.27 & \\
\hline
\end{tabular}

\subsection{Simulation of Actual Eluate Compositions}

Model predictions were also compared to OLI/ESP simulations using an expanded chemistry model based on all species present in the eight radioactive Tc ion exchange eluate samples generated from Hanford tank sample process studies conducted by Battelle and SRTC (those used to determine the significant species) above the minimum detection limits.

Simulations of these eight compositions were done at $20,35.369,35.37$, and $70^{\circ} \mathrm{C}$, corresponding to the two temperature ranges, for a total of 32 simulations. Table 4-5 lists the percent difference between the simulation results and the results form of the model whose predictions were in closest agreement ( 88 design points fit to the linear composition form). The ability of the trial expression to predict the simulation results of actual eluate compositions are not as good as their predictions of the of OLH design points using the simplified chemistry model. However, the many of the predictions are within the acceptance criteria for the upper temperature range (and for density in lower temperature range). The models need to be validated by results from the evaporation of simulant Tc eluate currently in progress.

Table 4-5. Comparison between Model and Simulation Results of Actual Eluate Compositions in terms of Percent Difference

\begin{tabular}{|c|c|c|c|c|c|c|}
\hline Sample & $\begin{array}{l}\text { Temp. } \\
\left({ }^{\circ} \mathrm{C}\right)\end{array}$ & $\begin{array}{c}\text { WMF } \\
\text { percent difference }\end{array}$ & $\begin{array}{c}\text { solubility } \\
\text { percent difference }\end{array}$ & $\begin{array}{c}\text { density } \\
\text { percent difference }\end{array}$ & $\begin{array}{l}\text { volume reduction } \\
\text { percent difference }\end{array}$ & $\begin{array}{c}\text { nonlinear } \\
\text { viscosity percent } \\
\text { difference }\end{array}$ \\
\hline \multicolumn{7}{|c|}{$20-35.369^{\circ} \mathrm{C}$ temperature range } \\
\hline AZ-102 sample 1 & 20 & -5.94 & 49.42 & -0.017 & -43.36 & 21 \\
\hline AZ-102 sample 2 & 20 & 38.13 & 50.82 & 0.0089 & -43.16 & 20 \\
\hline AN-105 sample 1 & 20 & 43.89 & -69.07 & -9.00 & -75.80 & 21 \\
\hline AN-105 sample 2 & 20 & 4.67 & -72.25 & -10.27 & -77.62 & 21 \\
\hline AN-107 sample 1 & 20 & 12.27 & -1.79 & 2.32 & -47.36 & 250 \\
\hline AN-107 sample 2 & 20 & 46.02 & -14.35 & 0.15 & -51.02 & 223 \\
\hline AN-103 sample 1 & 20 & 24.69 & -33.14 & -6.47 & -61.17 & 141 \\
\hline AN-103 sample 2 & 20 & -4.61 & -20.40 & -3.33 & -56.64 & 169 \\
\hline AZ-102 sample 1 & 35.369 & -4.63 & 43.09 & -1.73 & -44.87 & -16 \\
\hline AZ-102 sample 2 & 35.369 & -5.47 & 44.29 & -1.71 & -44.71 & -17 \\
\hline AN-105 sample 1 & 35.369 & -55.24 & 0.35 & 7.27 & -36.35 & 222 \\
\hline AN-105 sample 2 & 35.369 & 3.249 & 226.05 & 18.48 & -1.13 & 467 \\
\hline \begin{tabular}{|l|}
$\mathrm{AN}-107$ sample 1 \\
\end{tabular} & 35.369 & 11.05 & 0.48 & -1.06 & -48.66 & 126 \\
\hline AN-107 sample 2 & 35.369 & 31.80 & -12.66 & -2.83 & -52.11 & 109 \\
\hline AN-103 sample 1 & 35.369 & 11.86 & -19.99 & -6.34 & -57.83 & 87 \\
\hline AN-103 sample 2 & 35.369 & -5.93 & -4.39 & -3.35 & -53.10 & 110 \\
\hline \multicolumn{7}{|c|}{$35.37-70^{\circ} \mathrm{C}$ temperature range } \\
\hline AZ-102 sample 1 & 35.37 & 16.00 & -35.53 & -6.67 & -67.58 & -1.83 \\
\hline AZ-102 sample 2 & 35.37 & 16.12 & -35.74 & -6.72 & -67.66 & -2.07 \\
\hline AN-105 sample 1 & 35.37 & -1.47 & 3.87 & 2.06 & -45.29 & 211.80 \\
\hline AN-105 sample 2 & 35.37 & -1.30 & 3.06 & 2.16 & -45.87 & 208.18 \\
\hline AN-107 sample 1 & 35.37 & -11.38 & 28.52 & 3.15 & -41.25 & 101.37 \\
\hline AN-107 sample 2 & 35.37 & -9.53 & 23.42 & 2.47 & -42.8 & 93.80 \\
\hline AN-103 sample 1 & 35.37 & -13.97 & 33.16 & 6.48 & -39.95 & 131.77 \\
\hline
\end{tabular}


WSRC-TR-2002-00319 Rev. 0

SRT-RPP-2002-00171

\begin{tabular}{|c|c|c|c|c|c|c|}
\hline Sample & $\begin{array}{l}\text { Temp. } \\
\left({ }^{\circ} \mathrm{C}\right)\end{array}$ & $\begin{array}{c}\text { WMF } \\
\text { percent difference }\end{array}$ & $\begin{array}{c}\text { solubility } \\
\text { percent difference }\end{array}$ & $\begin{array}{c}\text { density } \\
\text { percent difference }\end{array}$ & $\begin{array}{l}\text { volume reduction } \\
\text { percent difference }\end{array}$ & $\begin{array}{c}\text { nonlinear } \\
\text { viscosity percent } \\
\text { difference } \\
\end{array}$ \\
\hline AN-103 sample 2 & 35.37 & -13.47 & 32.04 & 6.01 & -39.98 & 130.59 \\
\hline AZ-102 sample 1 & 70 & 10.87 & -25.82 & -7.10 & -63.73 & -50.57 \\
\hline AZ-102 sample 2 & 70 & 11.00 & -26.05 & -7.15 & -63.8 & -50.67 \\
\hline AN-105 sample 1 & 70 & 3.66 & -9.459 & -3.93 & -52.97 & -15.80 \\
\hline AN-105 sample 2 & 70 & 3.85 & -10.18 & -3.83 & -53.46 & -16.04 \\
\hline AN-107 sample 1 & 70 & -5.55 & 13.05 & -2.68 & -48.24 & -27.14 \\
\hline AN-107 sample 2 & 70 & -3.86 & 8.87 & -3.17 & -49.46 & -29.00 \\
\hline AN-103 sample 1 & 70 & -7.16 & 15.86 & 0.02 & -47.29 & -20.23 \\
\hline AN-103 sample 2 & 70 & -6.98 & 15.60 & -0.30 & -47.1 & -20.13 \\
\hline
\end{tabular}

Figure 4-19 - Figure 4-28 below show plots of the simulation physical property (such as solubility) vs. the corresponding model prediction of the physical property for the both temperature ranges. Two error curves representing $\pm 15 \%$ of the model prediction are included in the plots to quantify the success of the models. If $67 \%$ (1-sigma) of the points lay with the error curves, then the models meet the acceptance criteria.

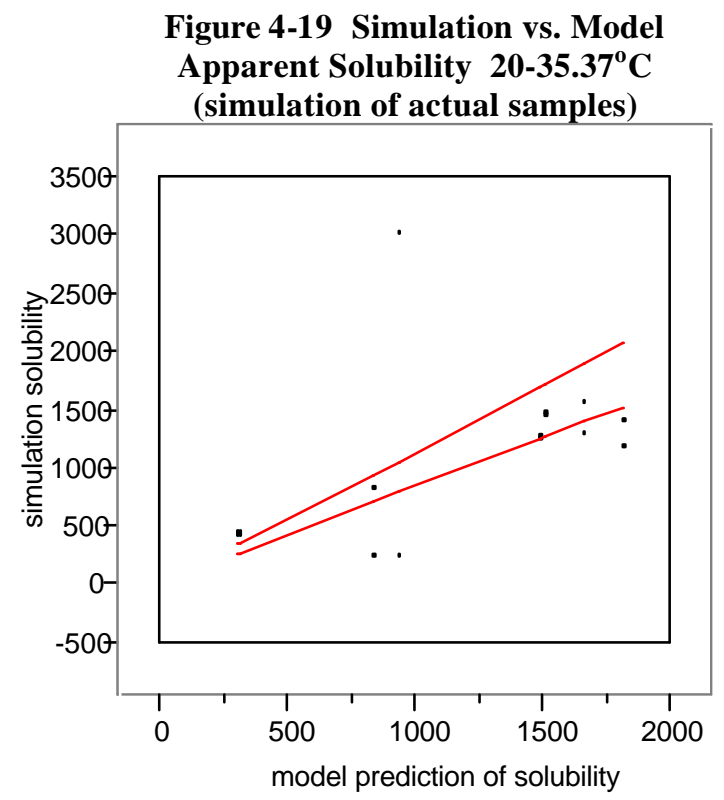

Plot of simulation vs. model apparent solubility for simulation of actual sample compositions. The two lines indicate the $\pm 15 \%$ error bounds of the model given by Eq. (9).

\section{Figure 4-20 Simulation vs. Model Water Mass 20-35.37 $\mathrm{C}$ (simulation of actual samples)}

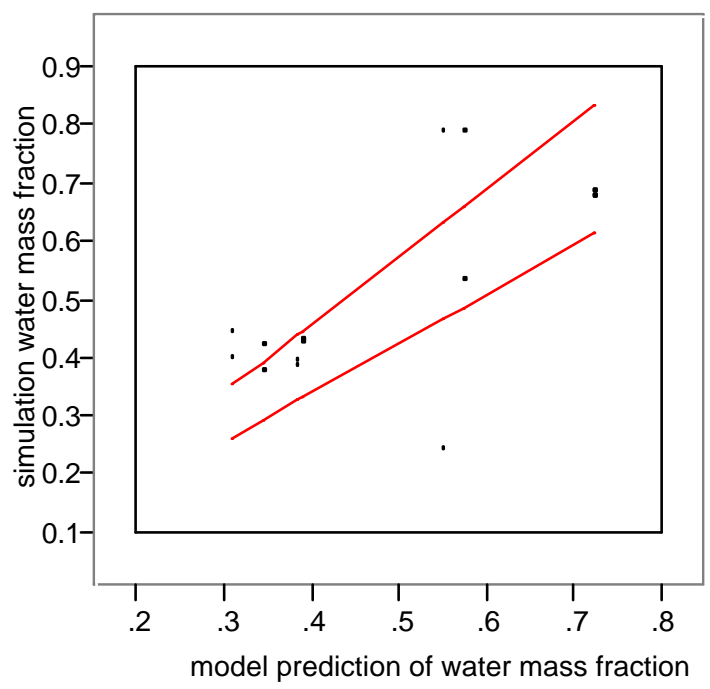

Plot of simulation vs. model water mass fraction for simulation of actual sample compositions. The two lines indicate the $\pm 15 \%$ error bounds of the model given by Eq. (10). 
Figure 4-21 Simulation vs. Model Density $20-35.37^{\circ} \mathrm{C}$ (simulation of actual samples)

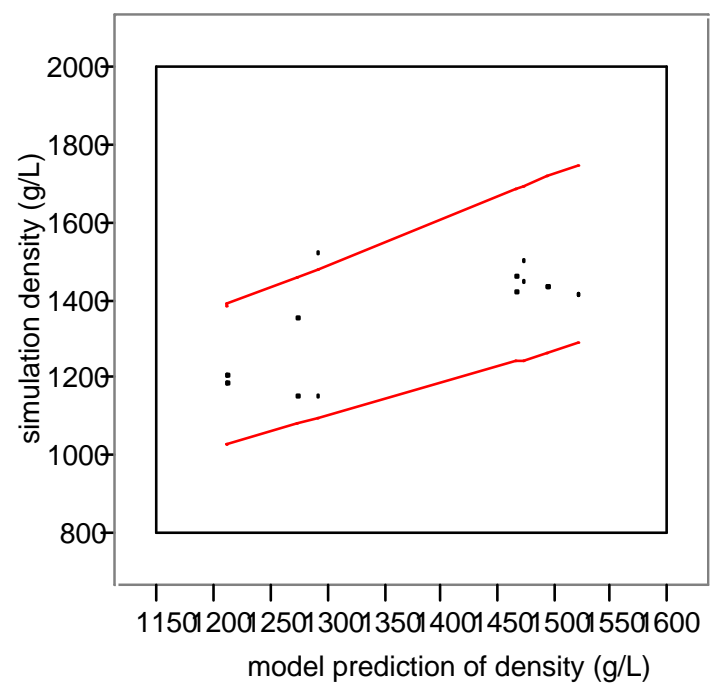

Plot of simulation vs. model density for simulation of actual sample compositions. The two lines indicate the $\pm 15 \%$ error bounds of the model given by Eq. (11).

Figure 4-23 Simulation vs. Model for Volume Reduction $20-35.37^{\circ} \mathrm{C}$ (simulation of actual samples)

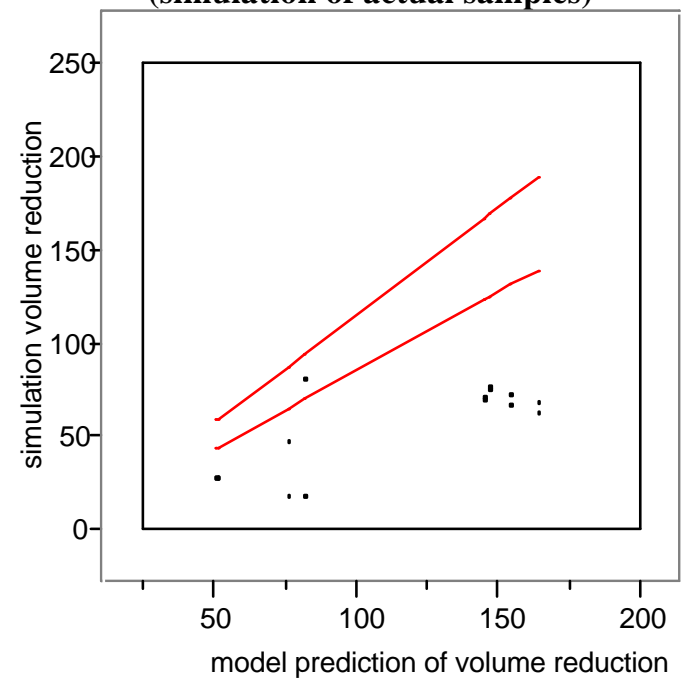

Plot of simulation vs. model volume reduction for simulation of actual sample compositions. The two lines indicate the $\pm 15 \%$ error bounds of the model given by Eq. (16).
Figure 4-22 Simulation vs. Nonlinear

Model Viscosity $20-35.37^{\circ} \mathrm{C}$

(simulation of actual samples)

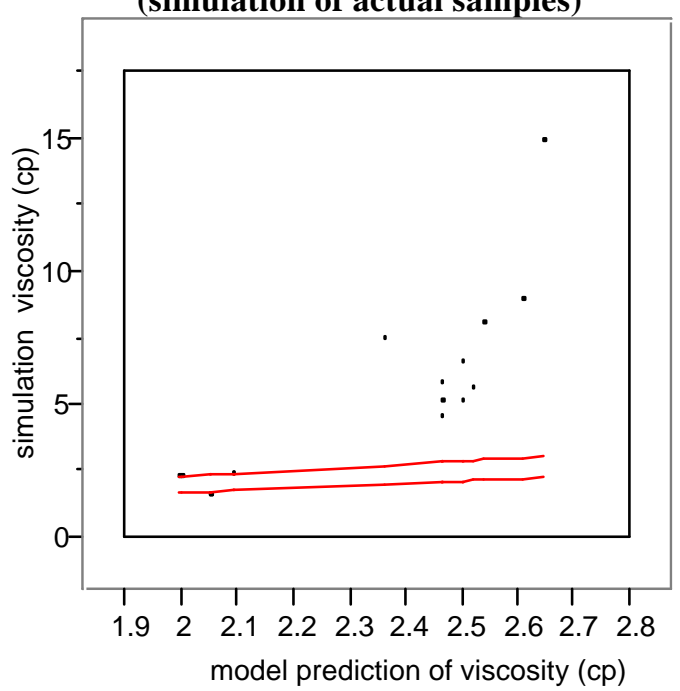

Plot of simulation vs. nonlinear viscosity model for simulation of actual sample compositions. The two lines indicate the $\pm 15 \%$ error bounds of the model given by Eq. (14).

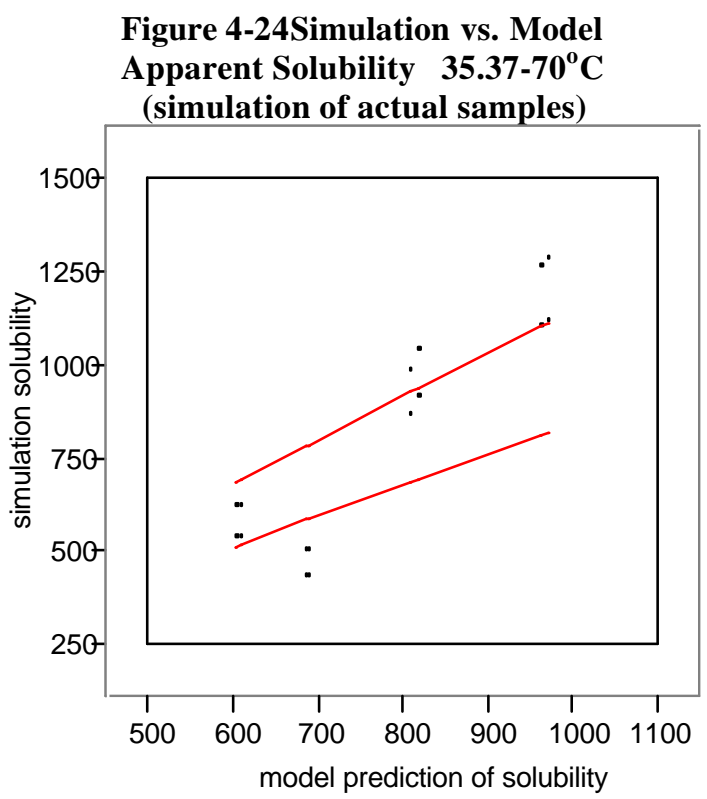

Plot of simulation vs. model apparent solubility for simulation of actual sample compositions. The two lines indicate the $\pm 15 \%$ error bounds of the model given by Eq. (1). 


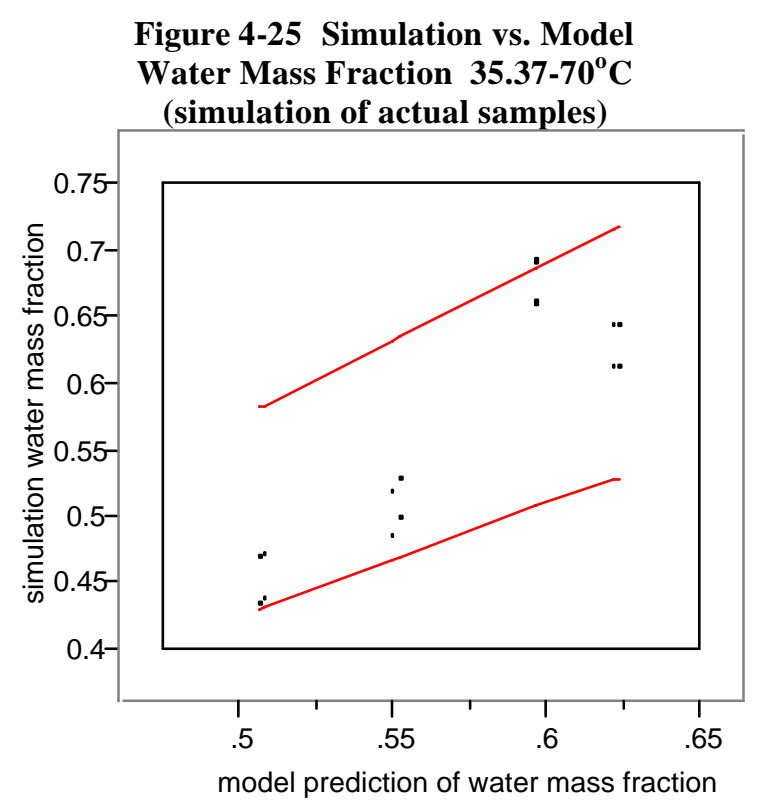

Plot of simulation vs. model water mass fraction for simulation of actual sample compositions. The two lines indicate the $\pm 15 \%$ error bounds of the model given by Eq. (2).

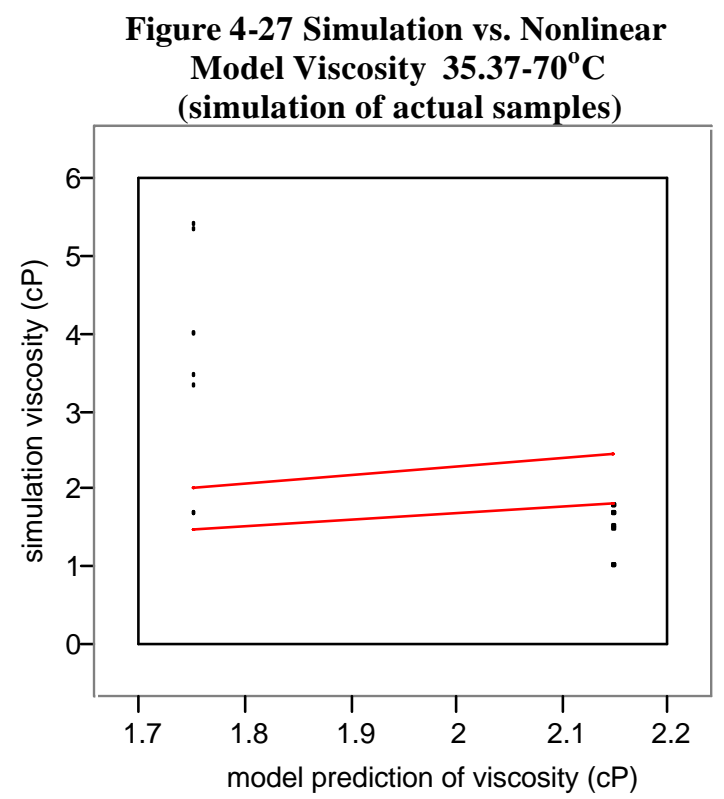

Plot of simulation vs. nonlinear viscosity model for simulation of actual sample compositions. The two lines indicate the $\pm 15 \%$ error bounds of the model given by Eq. (6).
Figure 4-26 Simulation vs. Model Density
$35.37-70^{\circ} \mathrm{C}$ (simulation of actual samples)

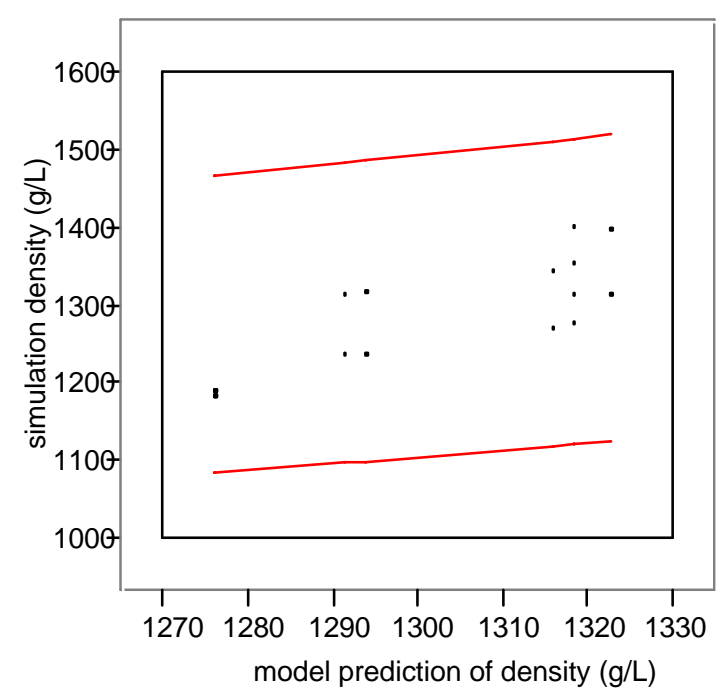

Plot of simulation vs. model density for simulation of actual sample compositions. The two lines indicate the $\pm 15 \%$ error bounds of the model given by Eq. (3).

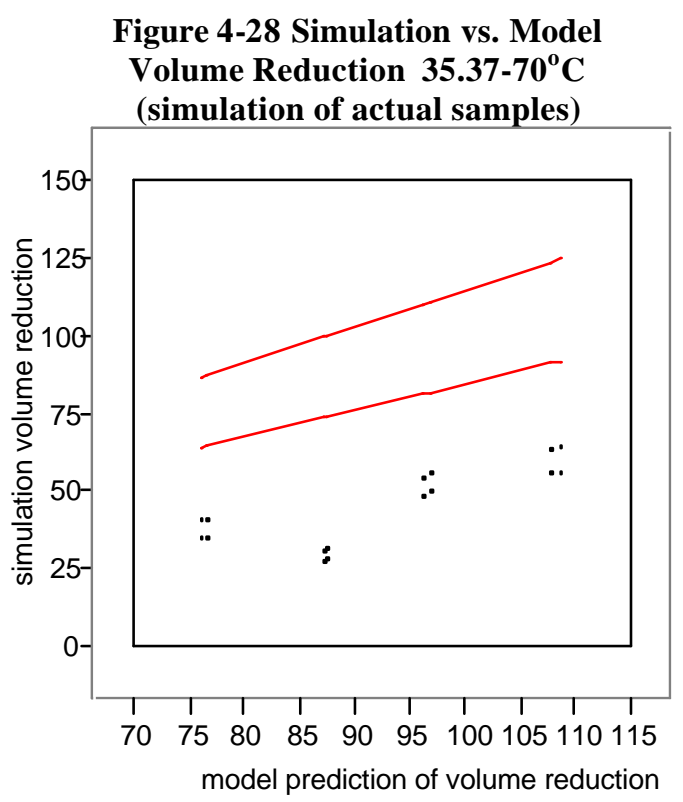

Plot of simulation vs. model volume reduction for simulation of actual sample compositions. The two lines indicate the $\pm 15 \%$ error bounds of the model given by Eq. (8). 


\section{Dynamic Simulation}

According to the WTP pretreatment flowsheet, the technetium eluate will be concentrated in a reboiler with thermo-siphon circulation to reduce the volume. The eluate is fed to the reboiler pot initially charged with water, and the vacuum is adjusted so that boilup would occur at $70{ }^{\circ} \mathrm{C}$ while maintaining a constant liquid volume in the pot. The feeding is to continue with no bottom removal until the liquid in the pot reaches the target endpoint defined earlier in this report. The $\mathrm{pH}$ of typical technetium eluate samples was estimated to be somewhere between 11 and 12, and a concern was raised by the WTP Process Engineering personnel over the potential of forming undesirable solids such as gibbsite during the initial phase of feeding, when a moderately alkaline eluate feed is trickled into a pool of neutral water.

The fact that water is the only volatile component of the technetium eluate has allowed us to approximate the semi-batch evaporation process described above as a continuous operation, since the same concentration endpoint would be reached regardless of whether the evaporation proceeds continuously or on a semi-batch mode. However, that same steady state approximation would not be valid, if one were to examine the impact of varying $\mathrm{pH}$ on the solubility of such salts as gibbsite or carbonate. This was precisely the motivation for the dynamic simulation of semi-batch evaporation discussed in this section.

\subsection{Dynamic Model}

The ESP software has a module, called DynaChem, used to simulate dynamic processes. However, its use as a full-scale dynamic flowsheet simulator is rather limited, since it provides three built-in "units" that can be used to model only a certain number of unit operations and does not allow users to build custom models. As a result, the main steady state module of the ESP software was used in this study to model the semibatch evaporator by approximating it as a series of continuous still pots, as shown in Figure 1. The mass ratio of the initial water charge-to-technetium eluate feed to the $1^{\text {st }}$ stage was set at 5:1. Additional stages were then added to the existing model one-by-one at the same feed ratio of 5:1, until the concentrate from the final stage reaches the prescribed evaporation endpoint at $25{ }^{\circ} \mathrm{C}$ and $1 \mathrm{~atm}$. Higher feed ratios of 10:1 and 100:1 were also tried and they were shown to have little impact on the overall vapor-liquid equilibria; only the required number of stages were increased proportionally. The validity of approximating the semibatch evaporator as a series of continuous still pots was confirmed earlier against the batch distillation data collected at $1 \mathrm{~atm}[13]$

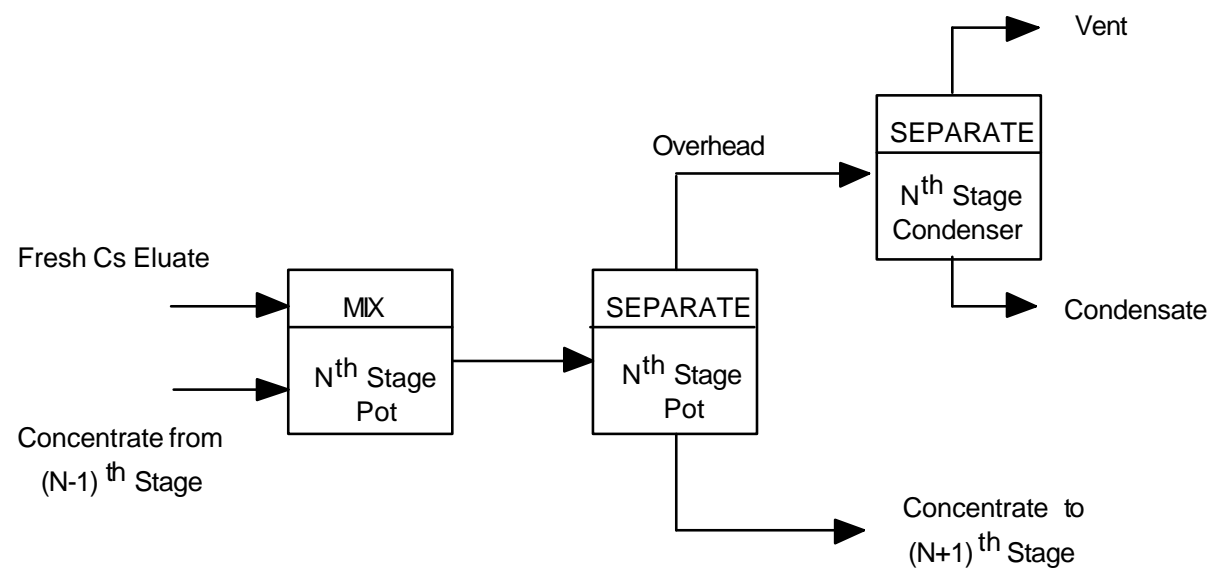

FIGURE 5-1. Schematic of $\mathbf{N}^{\text {th }}$ Stage Evaporation of Tc Eluate Model [Ref. 13]. 


\subsection{AZ-102 Technetium Eluate}

It was preferable to use the feed with the highest concentration of aluminum for the dynamic simulation, since the WTP Process Engineering personnel was most concerned with the possible formation of gibbsite when the $\mathrm{pH}$ of the solution in the pot is at its minimum. In Appendix B, the concentration of aluminate ion in the AZ-102 technetium eluate sample is estimated to be 0.0285 in mass fraction among all the anions considered, and Table 1-1 shows that this value is close to the upper limit of 0.03 for aluminate ion used in the matrix development. For this reason, the composition of AZ-102 technetium eluate shown in Table 5-1 was used as the feed to the dynamic model.

The composition shown in Table 5-1 was derived from the analytical data collected during the AZ-102 technetium ion-exchange column run at SRTC [7]. Since the free hydroxide concentration was not measured, it was instead estimated to be $0.0029 \mathrm{M}$ by performing a mass balance over the entire ionexchange run cycle. Furthermore, the reported value of total organic carbon (TOC) was reduced by a factor of 3.5, since the TOC level of the as-received AZ-102 sample measured by SRTC was higher than the grab sample data measured at the Hanford site in 1995 by the same factor [17]. As a result, the measured concentration of oxalate had to be reduced by $26 \%$ from 214 to $159 \mathrm{mg} / \mathrm{L}$ in order to match the adjusted TOC data, assuming that there were no other organic species present in the technetium eluate besides the oxalate. Finally, the concentration of sodium shown in Table 5-1 reflects approximately 18\% reduction from the measured data to preserve the charge balance. It is noted that the resulting mass fraction of aluminate ion in the adjusted AZ-102 technetium eluate was 0.0292 , which is slightly higher than the value estimated in Appendix B.

The actual input to the semi-batch evaporator model were the full-scale flow rates given in the right half of Table 5-1. These flow rates were set based on the instantaneous flow rate of sodium required to meet the design basis LAW glass production rate and the sodium-to-technetium mass ratio found in the untreated AZ-102 filtrate [7]. Based on the design basis Envelope B glass production rate of 60 metric tons per day at $10 \mathrm{wt} \% \mathrm{Na}_{2} \mathrm{O}$, the required instantaneous flow rate of sodium in the AZ-102 supernate was calculated to be $185,484 \mathrm{~g} / \mathrm{hr}$. The corresponding instantaneous flow rate of technetium was then calculated to be 47.9 $\mathrm{g} / \mathrm{hr}$ based on the sodium-to-technetium mass ratio of 3,872 , which was estimated from the SRTC analytical data [7]. Therefore, the full-scale flow rates given in Table 5-1 represent the required instantaneous flow rates of individual technetium eluate components to support the design basis Envelope B glass production rate.

TABLE 5-1. Composition of AZ-102 Technetium Eluate Feed for Semi-Batch Evaporation Model

\begin{tabular}{|c|c|c|c|c|c|c|c|c|}
\hline Anions & $\mathrm{FW}$ & Conc (mg/l) & Conc (M) & \multicolumn{2}{|c|}{ Equiv (M) wt frac anion } & Species & $\begin{array}{r}\text { full-scale flow } \\
\text { (mole/hr) }\end{array}$ & wt\% (dry) \\
\hline NO2 & 46 & 579.5 & 0.01260 & 0.01260 & 0.18325 & $\mathrm{NaNO} 2$ & $1.5316 \mathrm{E}+01$ & $1.7689 \mathrm{E}+01$ \\
\hline NO3 & 62 & 695.5 & 0.01122 & 0.01122 & 0.21994 & $\mathrm{NaNO} 3$ & 1.1188E+01 & $1.5917 \mathrm{E}+01$ \\
\hline $\mathrm{OH}$ & 17 & 49.3 & 0.00290 & 0.00290 & 0.01559 & $\mathrm{NaOH}$ & $3.5256 \mathrm{E}+00$ & $2.3647 \mathrm{E}+00$ \\
\hline $\mathrm{SO} 4$ & 98.058 & 596.5 & 0.00608 & 0.01217 & 0.18863 & $\mathrm{Na} 2 \mathrm{SO} 4$ & 7.3955E+00 & $1.7583 \mathrm{E}+01$ \\
\hline $\mathrm{C} 2 \mathrm{O} 4$ & 88.02 & 159.104 & 0.00181 & 0.00362 & 0.05031 & $\mathrm{Na} 2 \mathrm{C} 2 \mathrm{O} 4$ & $2.1976 \mathrm{E}+00$ & $4.9290 \mathrm{E}+00$ \\
\hline $\mathrm{AlO} 2$ & 58.98154 & 92.4676 & 0.00157 & 0.00157 & 0.02924 & $\mathrm{NaAlO} 2$ & $1.9060 \mathrm{E}+00$ & $2.6151 \mathrm{E}+00$ \\
\hline $\mathrm{CO} 3$ & 60.009 & 925 & 0.01541 & 0.03083 & 0.29251 & $\mathrm{Na} 2 \mathrm{CO} 3$ & $1.8740 \mathrm{E}+01$ & $3.3249 \mathrm{E}+01$ \\
\hline $\mathrm{TcO} 4$ & 162.9064 & 64.8948 & 0.00040 & 0.00040 & 0.02052 & NaTcO4 & 4.8430E-01 & $1.5069 \mathrm{E}+00$ \\
\hline \multirow[t]{2}{*}{ Total Anions } & & & 0.05199 & 0.07529 & 1.00000 & KNO3 & $2.4502 \mathrm{E}+00$ & $4.1463 \mathrm{E}+00$ \\
\hline & & & & & & $\mathrm{H} 2 \mathrm{O}$ & $6.8146 \mathrm{E}+04$ & \\
\hline Cations & & & & & & Total & $6.8210 \mathrm{E}+04$ & $1.0000 \mathrm{E}+02$ \\
\hline $\mathrm{Na}$ & 22.99 & 1684.65 & 0.07328 & 0.07328 & & & & \\
\hline $\mathrm{K}(\mathrm{AA})$ & 39.0983 & 78.80 & 0.00202 & 0.00202 & & & & \\
\hline Total Cations & & & 0.07529 & 0.07529 & & & & \\
\hline
\end{tabular}




\subsection{Execution of Dynamic Simulation Model}

One key process constraint that must be adhered to during the execution of the model was to ensure that the liquid volume in the pot or the flow rate of concentrate from one stage to the next is maintained constant throughout the entire evaporation cycle. This was achieved in essence by controlling the boilup rate or the vacuum in the pot. It turned out that by maintaining the molar boilup rate very close to that of the feed it was possible to contain the maximum volume fluctuations within $\pm 2 \%$.

The composition of concentrated eluate flow out of each stage was checked for presence of any solids, and the evaporation simulation was continued until the cumulative volume of eluate fed equaled 100 times (100X) the volume of water initially batched into the pot. The ESP model was run in conjunction with two private OLI databases, called GIBBSITE and CARBONAT, along with PUBLIC v6.5.

\subsection{Results of Dynamic Simulation}

The simulation results showed that the as-received AZ-102 technetium eluate feed would be at a $\mathrm{pH}$ of 11.7 , and $55 \%$ of aluminum would remain undissolved, i.e., as gibbsite. However, Figure 5-2 shows that as the eluate feed is introduced into the pot for the first time, all the gibbsite solids present in the feed would re-dissolve mainly due to dilution. The $\mathrm{pH}$ of the solution in the pot is shown to increase from 7 to above 11 instantly upon initiation of feeding. This means that the solution $\mathrm{pH}$ would remain above 11 practically throughout the evaporation cycle, and the concern over the $\mathrm{pH}$ being so low near neutral as to favor the formation of gibbsite appears unjustified.

Figure 5-2 further shows that as the concentration of aluminum in the pot increases with continued feeding, gibbsite begins to re-appear immediately and its concentration rapidly reaches its maximum when the cumulative feed volume equals about 10X the initial water volume. No more than $30 \%$ of total aluminum fed is predicted to remain as gibbsite during the entire evaporation cycle, and the total insoluble solids content in the pot due to gibbsite formation is practically negligible. However, as the concentration of gibbsite in the pot continuously declines, crystals of sodium oxalate begin to appear when the cumulative feed volume equals $30 \mathrm{X}$ the initial water volume. The total insoluble solids content in the pot only then begins to rise significantly. 


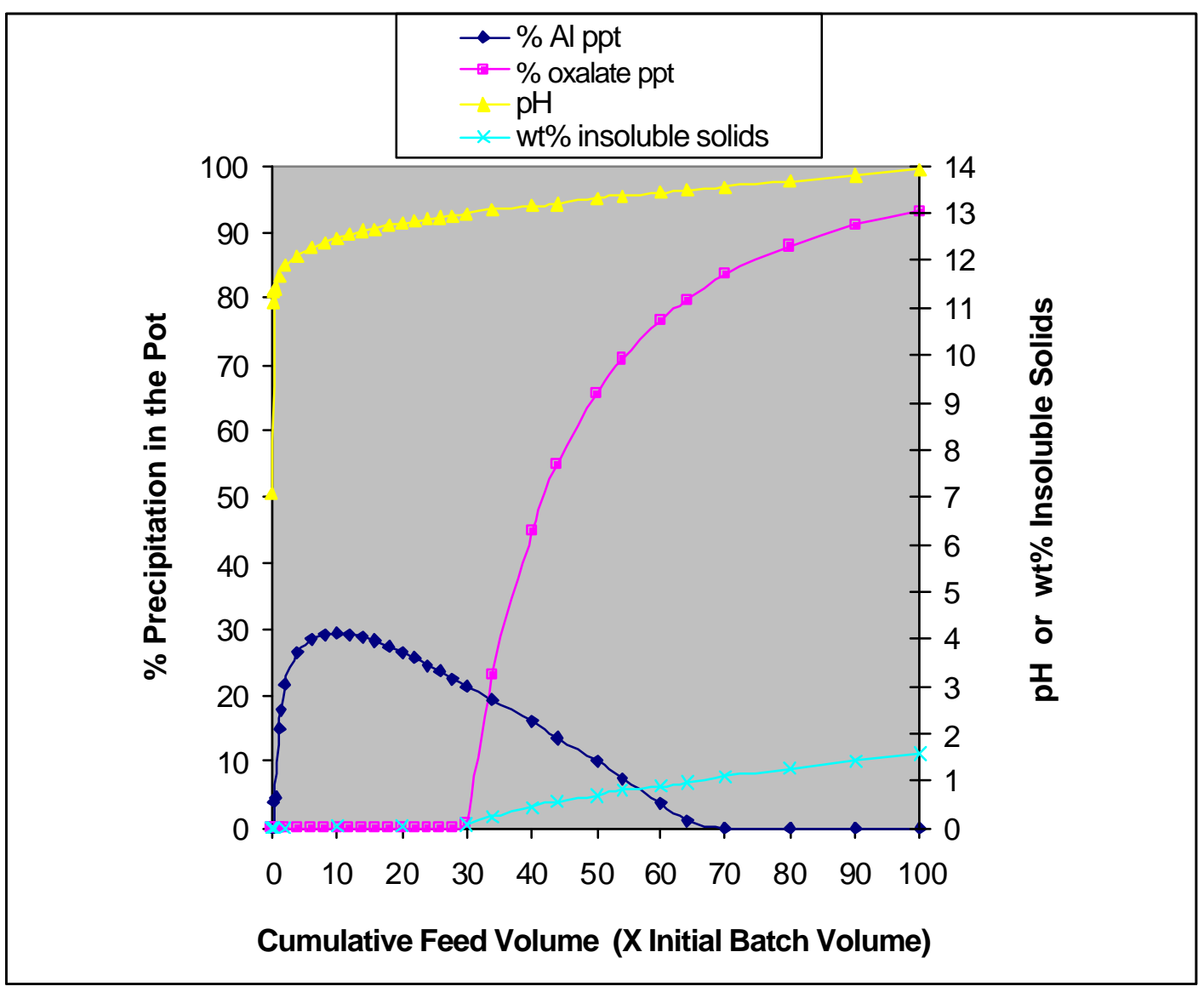

FIGURE 5-2. Results of Dynamic Simulation of Semi-Batch Evaporation of AZ-102 Technetium Eluate.

The model predicted that a total of 324 stages like the ones shown in Figure 5-1 would be required to reach the target endpoint of $1.0 \mathrm{wt} \%$ insoluble solids in the pot, consisting exclusively of sodium oxalate crystals. According to Figure 5-2, this would occur at a $\mathrm{pH}$ of 13.5 or when the cumulative feed volume equals $65 \mathrm{X}$ the initial water volume. Since the liquid volume in the pot was set to remain constant throughout the evaporation cycle, the volume reduction factor that would be achieved at the $1.0 \mathrm{wt} \%$ insoluble solids endpoint would also be $65 \mathrm{X}$. It is noted that the volume reduction factor is defined here as the ratio of the cumulative feed volume to the initial water volume. The model also predicted that the likelihood of forming any major salts such as $\mathrm{NaNO}_{3}, \mathrm{Na}_{2} \mathrm{CO}_{3} \cdot \mathrm{xH}_{2} \mathrm{O}$ or more importantly $\mathrm{NaTcO} 4$, out of the AZ-102 technetium eluate solution would still be remote even at $100 \mathrm{X}$ volume reduction.

\section{Conclusion/Summary}

Mathematical expressions for the solubility, density, heat capacity, viscosity and volume reduction were developed by fitting the results of computer simulations to several trial expressions. With the exception of the linear form for viscosity, all expressions derived for the $35.37-70^{\circ} \mathrm{C}$ range were well within acceptance criteria. Only the expression for density was within the acceptance criteria for the $20-35.37^{\circ} \mathrm{C}$ range, all others were significantly outside the acceptance criteria. This was due to the number of precipitating salts, as well as the complicated nature of their precipitation in the temperature range. 


\section{WSRC-TR-2002-00319 Rev. 0 \\ SRT-RPP-2002-00171}

Future work to improve the model fit in the $20-35.37^{\circ} \mathrm{C}$ range would include 1) an increased number of design points for higher resolution, 2) possible alternative functional forms for the physical properties, and 3) modification of the OLI/ESP databooks based on the analytical results of Tc eluate simulant work currently in progress.

Agreement between OLI/ESP simulations of actual Tc eluate composition and the mathematical models were generally not as good as that between those for simulations of the 41 OLH test points using the simplified OLI/ESP chemistry model and the mathematical models, although many were within specification.

The verification done here only tests the ability of the mathematical models to predict the OLI/ESP simulation results. While this is certainly a necessary test, it is of course insufficient. The models will be verified using data from simulant work, which is currently in progress. Ideally, actual Tc eluate evaporation tests would be the ultimate answer for testing any model.

The results of dynamic simulation showed that in the case of AZ-102 technetium eluate evaporation up to $30 \%$ of total aluminum fed could remain undissolved in the pot as gibbsite. However, the formation of gibbsite would not be an operational issue due to its low concentration. Instead, the target evaporation endpoint of $1.0 \mathrm{wt} \%$ insoluble solids in the pot would be reached due to formation of sodium oxalate crystals, and the maximum volume reduction factor that can be achieved at that endpoint is $65 \mathrm{X}$. Furthermore, the likelihood of forming any major salts such as $\mathrm{NaNO}_{3}, \mathrm{Na}_{2} \mathrm{CO}_{3} \cdot \mathrm{xH}_{2} \mathrm{O}$ (where $\mathrm{x}$ is 1 , 7, or 10 for the mono-, hepta-, or deca-hydrated salt) or more importantly NaTcO4, out of the AZ-102 technetium eluate solution would still be remote even at 100X volume reduction.

\section{References}

[1] Choi, A. S., "Task Technical and Quality Assurance Plan for Cesium and Technetium Eluate Physical Property Modeling”, WSCR-TR-2002-00408, Rev. 0, Westinghouse Savannah River Co., Aiken, SC, Nov. 2001.

[2] Edwards, T. B., "A Statistically Designed Test Matrix for a Computer Study of Tc Eluate Solubility”, SRT-SCS-2002-00030, Westinghouse Savannah River Co., Aiken, SC, May 2002.

[3] Ye, K.Q., "Orthogonal Column Latin Hypercubes and Their Application in Computer Experiments", Journal of the American Statistical Association, 93, 1430-1439, 1998.

[4] Hassan, N. M. et al, "Small-Scale Ion Exchange Removal of Cesium and Technetium from Hanford Tank 241-AN-103”, BNF-003-98-0146, Rev. 1, Westinghouse Savannah River Co., Aiken, SC, April 2000.

Hassan, N. M., McCabe, D. J., "Hanford Envelope B Tank Waste Ion Exchange Column Study", STRC-BNFL-017, Rev. 0, Westinghouse Savannah River Co., Aiken, SC, Oct. 1997.

Hassan, N. M., McCabe, D. J., "Hanford Envelope C Tank Waste Ion Exchange Column Study (U)”, STRC-BNFL-018, Rev. 0, Westinghouse Savannah River Co., Aiken, SC, Oct. 1997.

Hassan, N. M. et al, "Small-Scale Ion Exchange Removal of Cesium and Technetium from Envelope B Hanford Tank 241-AZ-102", WSRC-TR-2000-00419, Rev. 0, Westinghouse Savannah River Co., Aiken, SC, Jan. 2001.

Blanchard Jr., D. L., et al, "Small Column Testing of SuperLig ${ }^{\circledR} 639$ for Removing ${ }^{99}$ Tc from Hanford Tank Waste Envelope A (Tank 241-AW-101)", PNWD-3004, Rev. 0, Battelle, Pacific Northwestern Division, Richland, Washington, Aug. 2000. 
[9] Hassan, N. M. et al, "Small-Scale Ion Exchange Removal of Cesium and Technetium from Hanford Tank 241-AN-102", BNF-003-98-0219, Rev. 0, Westinghouse Savannah River Co., Aiken, SC, March 2000.

[10] King, W. D., et al, "Intermediate-Scale Ion Exchange Removal of Cesium and Technetium from Hanford Tank 241-AN-102", WSRC-TR-2000-00420, Rev. 0, Westinghouse Savannah River Co., Aiken, SC, Dec. 2000.

[11] Blanchard Jr., D. L., et al, "Small Column Testing of SuperLig ${ }^{\circledR} 639$ for Removing ${ }^{99}$ Tc from Hanford Tank Waste Envelope C (Tank 241-AN-107)", Battelle, Pacific Northwestern Division, Richland, Washington, June 2000.

[12] King, W. D., Calloway, T. B., "Tank 241-AZ-102 SuperLig ${ }^{\circledR} 639$ Technetium Ion Exchange Eluate Evaporation Study”, WSRC-TR-2000-00424, Rev. 0, Westinghouse Savannah River Co., Aiken, SC, 2000

[13] Choi, A. S., "Estimation of Physical Properties of AN-107 Cesium and Technetium Eluate Blend", WSRC-TR-2000-00527, Westinghouse Savannah River Co., Aiken, SC, Feb. 2001.

[14] Choi, A. S., "Software Quality Assurance Plan for Hanford RPP-WTP Evaporator Modeling (U)" WSRC-RP-2001-00337 Rev.0, Westinghouse Savannah River Co., Aiken, SC, Feb. 2001.

[15] SAS Institute, Inc., JMP ${ }^{\circledR}$ Statistics and Graphics Guide, Version 4.05, SAS Institute, Inc., Cary, NC, 2000.

[16] Hay, M. S., Bronikowski, M. G., "Chemical Characterization of an Envelope B/D Sample from Hanford Tank 241-AZ-102, BNF-003-98-0249 Rev. 0, Westinghouse Savannah River Co., Aiken, SC, July 2000

[17] Schreiber, Ruth D., "Tank Characterization Report for Double-Shell Tank 241-AZ-102" WHCSD-WM-ER-411 Rev. 0-A, Westinghouse Hanford Company, Richland, WA, Dec. 1995 


\section{Appendix A Compositions of Eluate Samples}

Table A-1lists the mass fractions of the significant anions for the actual Tc eluate samples. These values were used in the models to predict physical properties and compare with OLI/ESP simulations using the expanded chemistry model as described in Sec. 4.3. Table A-2lists the complete composition of the eight eluate samples.

Table A-1 Anion Mass Fractions of Actual Tc Eluate Samples

\begin{tabular}{|c|c|c|c|c|c|c|c|c|c|}
\hline & $\begin{array}{l}\mathrm{AlO} 2 \\
\text { mass fraction }\end{array}$ & \begin{tabular}{|l|}
$\mathrm{C} 2 \mathrm{O} 4$ \\
Mass fraction
\end{tabular} & \begin{tabular}{|l|}
$\mathrm{CO} 3$ \\
mass fraction
\end{tabular} & $\begin{array}{l}\mathrm{NO} 2 \\
\text { mass fraction }\end{array}$ & $\begin{array}{l}\text { NO3 } \\
\text { Mass fraction }\end{array}$ & \begin{tabular}{|l|}
$\mathrm{OH}$ \\
mass fraction
\end{tabular} & $\begin{array}{l}\mathrm{SO} 4 \\
\text { mass fraction }\end{array}$ & \begin{tabular}{|l|}
$\mathrm{TcO} 4$ \\
mass fraction
\end{tabular} & sum \\
\hline AZ-102 sample 1 & 0.030154 & 0.020397 & 0.300772 & 0.188918 & 0.227179 & 0.016351 & 0.195145 & 0.021085 & 1 \\
\hline AZ-102 sample 2 & 0.030205 & 0.020336 & 0.301494 & 0.189325 & 0.226819 & 0.016301 & 0.19423 & 0.021289 & 1 \\
\hline AN-105 sample 1 & 0.022209 & 0 & 0.806454 & 0.038727 & 0.105552 & 0.018221 & 0 & 0.008838 & 1 \\
\hline AN-105 sample 2 & 0.017431 & 0 & 0.79688 & 0.04157 & 0.116835 & 0.018398 & 0 & 0.008886 & 1 \\
\hline AN-107 sample 1 & 0.021071 & 0 & 0.219534 & 0.359151 & 0.359182 & 0.040628 & 0 & 0.000434 & 1 \\
\hline AN-107 sample 2 & 0.020874 & 0 & 0.235019 & 0.352456 & 0.352487 & 0.03875 & 0 & 0.000416 & 1 \\
\hline AN-103 sample 1 & 0.007519 & 0 & 0.16776 & 0.061037 & 0.732511 & 0.022569 & 0 & 0.008603 & 1 \\
\hline AN-103 sample 2 & 0.015272 & 0 & 0.173612 & 0.065022 & 0.714764 & 0.02285 & 0 & 0.008481 & 7 \\
\hline
\end{tabular}

Mass fractions of significant anions of actual Tc eluate samples applied to

mathematical models to predict physical properties.

Table A-2. Composition of Radioactive Tc Eluate Samples above Minimum Detection Limits (Charge Balanced)

\begin{tabular}{|c|c|c|c|c|c|c|c|c|}
\hline ICP-AES & charge & AZ-102 Sample 1 & AZ-102 Sample 2 & AN-105 Sample 1 & AN-105 Sample 2 & AN-107 Sample 1 & AN-107 Sample 2 & AN-103 Sample 1 \\
\hline element & & mole/L & Mole/L & mole/L & mole/L & mole/L & mole/L & mole/L \\
\hline $\mathrm{Al}(\mathrm{AlO} 2)$ & -1 & 0.001564006 & 0.001571 & 0.00103 & 0.0008 & 0.000423 & 0.000439 & 0.000284 \\
\hline $\mathrm{B}$ & 3 & 0.000807511 & 0.000802 & 0.000771 & 0.000671 & 0.000303 & 0.000309 & -- \\
\hline $\mathrm{Ba}$ & 2 & $1.37625 \mathrm{E}-05$ & $1.41 \mathrm{E}-05$ & -- & \begin{tabular}{l|l}
- & \\
\end{tabular} & -- & -- & -- \\
\hline $\mathrm{Ca}$ & 2 & $7.58521 \mathrm{E}-06$ & $1.79 \mathrm{E}-05$ & $2.24 \mathrm{E}-05$ & 0.000102 & $4.93 \mathrm{E}-05$ & $4.9 \mathrm{E}-05$ & $2.54 \mathrm{E}-05$ \\
\hline $\mathrm{Cd}$ & 2 & $3.08691 \mathrm{E}-06$ & $3.97 \mathrm{E}-06$ & -- & -- & -- & $9.81 \mathrm{E}-07$ & $3.65 \mathrm{E}-06$ \\
\hline $\mathrm{Co}$ & 2 & $1.54582 \mathrm{E}-05$ & $1.83 \mathrm{E}-05$ & -- & -- & $8.85 \mathrm{E}-06$ & $8.28 \mathrm{E}-06$ & -- \\
\hline $\mathrm{Cr}(\mathrm{CrO} 4)$ & -2 & 0.0002981 & 0.000298 & $2.11 \mathrm{E}-05$ & -- & $6.69 \mathrm{E}-06$ & $8.24 \mathrm{E}-06$ & -- \\
\hline $\mathrm{Cu}$ & 2 & $1.0575 \mathrm{E}-05$ & $1.13 \mathrm{E}-05$ & -- & -- & $8.08 \mathrm{E}-06$ & $9.81 \mathrm{E}-06$ & -- \\
\hline $\mathrm{Fe}$ & 3 & $8.93513 \mathrm{E}-06$ & $1.01 \mathrm{E}-05$ & -- & -- & $2.77 \mathrm{E}-05$ & $2.7 \mathrm{E}-05$ & -- \\
\hline $\mathrm{K}(\mathrm{AA})$ & 1 & 0.001992429 & 0.002041 & 0.001465 & 0.00139 & 0.0002 & 0.000188 & -- \\
\hline \begin{tabular}{|l}
$\mathrm{La}$ \\
\end{tabular} & 3 & $1.15182 \mathrm{E}-05$ & $1.22 \mathrm{E}-05$ & $2.44 \mathrm{E}-05$ & -- & $4.02 \mathrm{E}-05$ & $4.67 \mathrm{E}-05$ & -- \\
\hline $\mathrm{Li}$ & 1 & 6.09422E-05 & 0.0002 & -- & -- & $8.86 \mathrm{E}-05$ & $9.53 \mathrm{E}-05$ & -- \\
\hline $\mathrm{Mg}$ & 2 & -- & -- & -- & $5.06 \mathrm{E}-06$ & $3.67 \mathrm{E}-06$ & $3.84 \mathrm{E}-06$ & -- \\
\hline $\mathrm{Mn}$ & 2 & $2.56653 \mathrm{E}-06$ & $2.97 \mathrm{E}-06$ & -- & -- & -- & -- & -- \\
\hline Mo (MoO4) & -2 & $1.78236 \mathrm{E}-05$ & $1.66 \mathrm{E}-05$ & -- & -- & -- & -- & $5.31 \mathrm{E}-06$ \\
\hline \begin{tabular}{|l|}
$\mathrm{Na}$ \\
\end{tabular} & 1 & 0.07881837 & 0.078947 & 0.082298 & 0.081075 & 0.031433 & 0.035178 & 0.045199 \\
\hline $\mathrm{Ni}$ & 2 & $1.95945 \mathrm{E}-05$ & $1.61 \mathrm{E}-05$ & -- & $1.59 \mathrm{E}-05$ & $3.33 \mathrm{E}-05$ & $3.69 \mathrm{E}-05$ & -- \\
\hline $\mathrm{P}$ (PO4) & -3 & $1.15182 \mathrm{E}-05$ & $1.22 \mathrm{E}-05$ & -- & -- & -- & -- & $4.88 \mathrm{E}-06$ \\
\hline $\mathrm{Pb}$ & 2 & $2.17664 \mathrm{E}-05$ & $2.3 \mathrm{E}-05$ & -- & $2.46 \mathrm{E}-05$ & $1.2 \mathrm{E}-05$ & -- & -- \\
\hline \begin{tabular}{|l|}
$\mathrm{Si}(\mathrm{HSiO} 4)$ \\
\end{tabular} & -1 & 0.000242114 & 0.00022 & 0.000972 & 0.000732 & 0.000833 & 0.000887 & 0.000171 \\
\hline $\mathrm{Sn}$ & 2 & -- & -- & -- & -- & $2.38 \mathrm{E}-05$ & $3.19 \mathrm{E}-05$ & -- \\
\hline $\mathrm{Ti}$ & 3 & $3.92605 \mathrm{E}-06$ & $4.03 \mathrm{E}-06$ & -- & -- & -- & -- & -- \\
\hline $\mathrm{U}$ & 4 & $2.71397 \mathrm{E}-07$ & $3.21 \mathrm{E}-07$ & -- & -- & -- & -- & -- \\
\hline \begin{tabular}{|l|}
$\mathrm{V}$ \\
\end{tabular} & 3 & $4.3692 \mathrm{E}-06$ & $4.62 \mathrm{E}-06$ & -- & -- & $3.44 \mathrm{E}-06$ & $3.78 \mathrm{E}-06$ & -- \\
\hline $\mathrm{Zn}$ & 2 & & -- & -- & -- & $3.77 \mathrm{E}-06$ & $3.53 \mathrm{E}-06$ & -- \\
\hline $\mathrm{Zr}$ & 4 & $4.29729 \mathrm{E}-05$ & $4.22 \mathrm{E}-05$ & -- & -- & -- & -- & -- \\
\hline \multicolumn{9}{|l|}{ IC } \\
\hline $\mathrm{Cl}$ & -1 & 0.009646574 & 0.009759 & 0.000628 & 0.000698 & 0.004265 & 0.006445 & -- \\
\hline $\mathrm{NO} 2$ & -1 & 0.012563579 & 0.012629 & 0.002302 & 0.002447 & 0.009243 & 0.009511 & 0.002954 \\
\hline $\mathrm{NO} 3$ & -1 & 0.011208773 & 0.011225 & 0.004654 & 0.005102 & 0.006858 & 0.007057 & 0.026303 \\
\hline $\mathrm{C} 2 \mathrm{O} 4$ & -2 & 0.00070893 & 0.000709 & -- & -- & -- & -- & -- \\
\hline SO4 & -2 & 0.006214607 & 0.006204 & -- & -- & -- & -- & -- \\
\hline $\mathrm{OH}$ & -1 & 0.002941176 & 0.002941 & 0.002929 & 0.002929 & 0.002828 & 0.002828 & 0.002955 \\
\hline TIC (CO3) & -2 & 0.015333333 & 0.015417 & 0.036744 & 0.035957 & 0.004331 & 0.004861 & 0.006224 \\
\hline \multicolumn{9}{|l|}{ GEA } \\
\hline Sr-90 & 2 & -- & -- & -- & -- & -- & -- & $8.06 \mathrm{E}-10$ \\
\hline \multicolumn{9}{|l|}{ Cs-137 } \\
\hline \multicolumn{9}{|l|}{ ICP-MS } \\
\hline $\mathrm{Tc}-99(\mathrm{TcO} 4)$ & -1 & 0.00039596 & $4.01 \mathrm{E}-04$ & 0.000148 & 0.000148 & $3.15 \mathrm{E}-06$ & $3.17 \mathrm{E}-06$ & $1.18 \mathrm{E}-04$ \\
\hline
\end{tabular}




\section{Appendix B Sample Calculation}

The following is an example calculation of the density using equation (3) for sample 1 of AZ-102. The concentrations, listed in Table B-2, are given in terms of mole/L, and are converted to a mass/L for the eight anions using the molecular weights given in Table B-1 From this the anion mass fractions are calculated and then applied to the equation for density.

Table B-1 Mass Fraction Ranges for which Models are Valid

\begin{tabular}{|c|c|c|c|c|c|c|c|c|}
\hline & $\mathrm{AlO}_{2}$ & $\mathrm{C}_{2} \mathrm{O}_{4}$ & $\mathrm{CO}_{3}$ & $\mathrm{NO}_{2}$ & $\mathrm{NO}_{3}$ & $\mathrm{OH}$ & $\mathrm{SO}_{4}$ & $\mathrm{TcO}_{4}$ \\
\hline $\mathrm{FW}$ & 58.982 & 88.02 & 60.0093 & 46.002 & 62.005 & 17.007 & 96.064 & 162.9039 \\
\hline $\mathrm{Min}$ & 0.00750 & 0.00001 & 0.16500 & 0.03900 & 0.10500 & 0.01600 & 0.000001 & 0.00042 \\
\hline Max & 0.03000 & 0.02050 & 0.80500 & 0.36000 & 0.73500 & 0.04100 & 0.19500 & 0.02150 \\
\hline \multicolumn{7}{|c|}{ Valid ranges for anions in terms of mass fractions relative total mass of the eight anions. }
\end{tabular}

Table B-2. Composition of AZ-102, Sample 1

\begin{tabular}{|c|c|c|c|c|c|c|c|c|}
\hline & $\mathrm{AlO}_{2}$ & $\mathrm{C}_{2} \mathrm{O}_{4}$ & $\mathrm{CO}_{3}$ & $\mathrm{NO}_{2}$ & $\mathrm{NO}_{3}$ & $\mathrm{OH}$ & $\mathrm{SO}_{4}$ & $\mathrm{TcO}_{4}$ \\
\hline $\mathrm{Mol} / \mathrm{L}$ & $1.56 \mathrm{E}-3$ & $0.709 \mathrm{E}-3$ & 0.0153 & 0.012 & 0.0112 & $2.94 \mathrm{E}-3$ & $6.21 \mathrm{E}-3$ & $0.396 \mathrm{E}-3$ \\
\hline
\end{tabular}

The total mass of the eight significant anions per liter is:

Total mass /liter of significant anions:

$=[\mathrm{AlO} 2] * 58.982+\left[\mathrm{C}_{2} \mathrm{O}_{4}\right] * 88.02+\left[\mathrm{CO}_{3}\right] * 60.0093+\left[\mathrm{NO}_{2}\right] * 46.002+\left[\mathrm{NO}_{3}\right] * 62.005+[\mathrm{OH}] * 17.007+$ $\left[\mathrm{SO}_{4}\right] * 96.064+\left[\mathrm{TcO}_{4}\right] * 162.9039$

$=[0.00156] * 58.982+[0.000709] * 88.02+[0.0153] * 60.0093+[0.012] * 46.002+[0.0112] * 62.005+$

$[0.00294] * 17.007+[0.00621] * 96.064+[0.000396] * 162.9039$

$=0.09024+0.0624+0.9181+0.552+0.694+0.05+0.597+0.0645$

$=3.028 \mathrm{~g} / \mathrm{L}$ (total mass of anions $/$ liter)

$$
\begin{aligned}
\mathrm{AlO} 2 & =0.09024 / 3.028=0.0298 \\
\mathrm{C} 2 \mathrm{O} 4 & =0.0624 / 3.028=0.0206 \\
\mathrm{CO} 3 & =0.9181 / 3.028=0.303 \\
\mathrm{NO} 2 & =0.552 / 3.028=0.182 \\
\mathrm{NO} 3 & =0.694 / 3.028=0.229 \\
\mathrm{OH} & =0.05 / 3.028=0.0165 \\
\mathrm{SO} 4 & =0.597 / 3.028=0.197 \\
\mathrm{TcO} 4 & =0.645 / 3.028=0.0212
\end{aligned}
$$

The density is calculated using the anion mass fractions and Eq. 3 for density in the $35.37-70^{\circ} \mathrm{C}$ range below.

$$
\begin{aligned}
& \text { density at endpoint conditions }(\mathrm{g} / \mathrm{L}) \\
& \begin{aligned}
\mathbf{1 , 9 2 0} *[\mathrm{AlO}]+1,150 *\left[\mathrm{C}_{2} \mathrm{O}_{4}\right]+1,260 & * \mathrm{CO}]+1,210 *[\mathrm{NO}] \\
& +1,300 *\left[\mathrm{NO}_{3}\right]+2,470 *[\mathrm{OH}]+1,180 *\left[\mathrm{SO}_{4}\right]+1,060 *[\mathrm{TcO}]
\end{aligned}
\end{aligned}
$$

$$
\begin{aligned}
& =1920 * 0.0298+1150 * 0.0206+1260 * 0.303+1210 * 0.182+1300 * 0.229+2470 * 0.0165+1180 * 0.197+ \\
& 1060 * 0.0212 \\
& =57.22+29.9+381.78+220.22+297.7+40.76+232.46+22.47 \\
& =1282.51(\mathrm{gram} / \mathrm{L})
\end{aligned}
$$




\section{Appendix C Quality Assurance; Verification of Excel Macros and Perl Script}

Note, the following examples were done using results from fits to a single temperature range $\left(20-70^{\circ} \mathrm{C}\right)$. Although the data does not correspond to the results presented in this work, the example is still valid as the identical macros and scripts were used in both cases.

\section{C.1 Excel Macros}

Two Excel macros were used to automate the execution of OLI/ESP Tc eluate simulations, one for simulations of evaporation of the eluate to the endpoint composition and temperature, and the other for generating enthalpytemperature plots for heat capacity calculations.

In both cases, the macros modify an existing OLI/ESP input file (with suffix "bin") prior to the execution of a batch program ("batrun.exe", written by OLI at the request of Frank Smith of SRTC) which reads the names of the OLI/ESP chemistry model and flow sheet from a text file ("names.lis") and passes them to the OLI/ESP simulation engine for execution. Finally, the OLI/ESP input and output files (with suffixes "bin", "bou", and "bst") are copied to a specified directory at the completion of the simulation. The names of the copied files were made unique by appending them with the design point "run number", providing a cross-reference between the output file and corresponding design point.

The macro used for the endpoint simulations writes the OLI species name and composition (read from a spreadsheet) to an existing OLI/ESP input file, along with an initial guess of the vapor mole fraction to be removed to achieve the endpoint. At the completion of the simulation, the "bst" output file was read to determine the weight percent solids of the product stream or if a sodium nitrate or nitrite salt has precipitated. The vapor fraction is adjusted accordingly and written to the "bin" input file for the next iteration of the simulation. This sequence continued until the endpoint is reached within a specified tolerance, when the simulation of the next design point is begun.

The macro for heat capacity calculations is similar, but instead of reading the input composition from a spreadsheet, it used the endpoint "bin" input file from the previous series of simulations described above. It ran a series of simulations over a specified temperature range by writing the appropriate temperature of the product stream to the "bin" file. At the completion of each simulation, the enthalpy of the product stream was copied into an exiting spreadsheet from the "bst" output file. Enthalpy-temperature plots for each design point were created from the data in this spreadsheet.

\section{C.2 Perl Script}

Perl is a well-known programming language widely used for file manipulation. A perl script was used to read relevant values (density, water mass, etc.) from the OLI/ESP output files (*.bst) and write them to text files for input to JMP ${ }^{\circledR}$, the statistical software used for the model fits. The Excel macro used to automate the simulations saved the OLI/ESP output files to a unique name by appending them with a "run number". This "run number" was read from the file name by the Perl script and used to cross-reference the retrieved simulation values with the appropriate design point. Each line of the generated text begins with the simulation run number, followed by the comma separated simulation results.

\section{C.3 Example Verification}

Note: the examples below are from the initial simulations done for the entire $20-70^{\circ} \mathrm{C}$ temperature range (as opposed to the split temperature ranges described in the body of the report), however, the examples are still valid as the identical macros and scripts were used in both cases.

This appendix gives an example of the type of verification that was done to insure that data used in JMP was correctly retrieve from the OLI/ESP output files. 


\section{WSRC-TR-2002-00319 Rev. 0 \\ SRT-RPP-2002-00171}

Table C.1 is a clip of the Excel file of the OLI/ESP results, retrieved from the OLI/ESP output files using the Excel macros and Perl script, and used by JMP ${ }^{\circledR}$ to fit the models. Table C.2 lists the enthalpies as a function of temperature, and was generated by the Excel macro. Tables C.3 and C.4 are partial listings of an OLI/ESP output file from run number TS001, and one of the files from which data was read. Table C.3 lists the details of the concentrated eluate stream (product), and Table C.4 lists data on the block (unit operation) used to set the temperature of the concentrated eluate to its endpoint. The following are calculations from the OLI/ESP output file to compare with the data in the Excel files.

The water mass fraction was calculated from the moles/hr of water, multiplied by its molecular weight of 18.01534 grams/mole, and divided by the mass/hr of the aqueous phase of the stream, and is:

$$
\begin{aligned}
& \text { water mass fraction }=3246.5(\mathrm{~mol} / \mathrm{hr}) * 18.01534(\mathrm{gram} / \mathrm{mol}) / 77996.3(\mathrm{gram} / \mathrm{hr}) \\
& =0.749866357,
\end{aligned}
$$

the same value found in Table C.1 within the significant digits used.

The density of the aqueous stream was read directly from the OLI/ESP output file as $1218.78 \mathrm{gram} / \mathrm{L}$, and is exactly the same as the density given in the Excel file.

The enthalpy per gram (used in the heat capacity calculations) is that of the aqueous and solids phases combined, and was calculated from the total of the aqueous and solid streams given in the OLI/ESP mixer block in Table C.4 (the individual aqueous and solid values listed are truncated at six digits, and so their sum is less accurate than the given total). From the OLI/ESP output file this is:

$$
\text { Enthalpy }(\mathrm{cal} / \mathrm{gram})=-2.74522 \mathrm{E}+08(\mathrm{cal} / \mathrm{hr}) / 78387(\mathrm{gram} / \mathrm{hr})=-3502.110028 \mathrm{cal} / \mathrm{gram} \text {, }
$$

\begin{tabular}{|c|c|c|c|c|}
\hline Run & $\begin{array}{l}\text { water } \\
\text { mass fract. }\end{array}$ & solubility & $\begin{array}{l}\text { density } \\
(\mathrm{g} / \mathrm{L})\end{array}$ & $\begin{array}{l}\text { Cp } \\
\mathrm{cal} / \mathrm{g} / \mathrm{C}\end{array}$ \\
\hline TSO01 & 0749866 & 0333571 & 121878 & 08052 \\
\hline TS002 & 0.451201 & 1216305 & 1437.44 & 0.5088 \\
\hline ISO03 & 0.378470 & 1.642216 & 1596.50 & 0.4723 \\
\hline TSO04 & 0.801382 & 0.247844 & 1177.49 & 0.828 \\
\hline S005 & 0.847942 & 0.179325 & 1096.27 & 0.867 \\
\hline Tsono & م848779 & 0178162 & 110238 & 0865 \\
\hline TSO07 & 0.874146 & 0.143973 & 1094.15 & 0.896 \\
\hline TSO08 & 0.877214 & 0.139973 & 1078.52 & 903 \\
\hline TS009 & 0.663816 & 0.506443 & 124128 & 0.703 \\
\hline TS010 & 0.320789 & 2.117310 & 1438.09 & 0.4076 \\
\hline Ts011 & 0623903 & 0602812 & 122153 & 0669 \\
\hline TS012 & 0.653656 & 0.529857 & 1254.51 & 0.6826 \\
\hline TS013 & 0.334497 & 1.989567 & 1414.22 & 0.43 \\
\hline ISO14 & 0.75 & 0.262904 & 1109.70 & 0.831 \\
\hline TS015 & 0.802111 & 0.246710 & 3.46 & 0.833 \\
\hline ISO16 & 080 & 0238546 & 109307 & 084 \\
\hline ISO17 & 0.68 & 0.454823 & 0.99 & 0.72 \\
\hline 018 & 0.791531 & 0.263374 & 1153.96 & 0.82 \\
\hline 019 & 0.678721 & 0.473359 & 1199.98 & 71 \\
\hline & & & & \\
\hline
\end{tabular}

exactly the same value as that in Table C.2

Table C-3. Simulation Results of First 20 Design Points 
WSRC-TR-2002-00319 Rev. 0

SRT-RPP-2002-00171

Table C-4. Enthalpies of First 4 Design Points

\begin{tabular}{|c|c|c|c|c|c|c|c|c|c|}
\hline $\begin{array}{r}\text { OLI Bin file } \\
\text { name/ TEMP }\end{array}$ & endpoint -0.8 & Endpoint -0.6 & Endpoint -0.4 & Endpoint -0.2 & $\begin{array}{r}\text { enthalpy at } \\
\text { endpoint temp } \\
\end{array}$ & endpoint +0.2 & Endpoint +0.4 & endpoint +0.6 & endpoint +0.8 \\
\hline TCELUAT1.bin & -3504.342524 & -3503.793967 & -3503.245411 & -3502.684098 & -3502.110028 & -3501.803857 & 38014 & 484929 & -3501.319086 \\
\hline TCELUAT2.bin & -2659.49329 & -2659.335839 & -2659.178389 & -2659.020938 & -2658.863487 & -2658.762269 & -2658.658239 & -2658.557021 & -2658.455803 \\
\hline TCELUAT3.bin & -2648.119749 & -2647.971138 & -2647.825688 & -2647.677077 & -2647.528465 & -2647.433607 & -2647.338749 & -2647.24389 & -2647.14903 \\
\hline TCELUAT4.bin & -3567.358865 & -3566.830934 & -3566.303004 & -3565.764921 & -3565.216685 & -3564.800432 & -3564.627839 & -3564.465399 & -3564.302959 \\
\hline
\end{tabular}

Enthalpies (cal/gram) at various temperatures about (and including) the endpoint temperature for design points 1-4.

Table C-5. Portion of OLI/ESP Simulation Output File for Design Point 1 Showing Eluate Stream

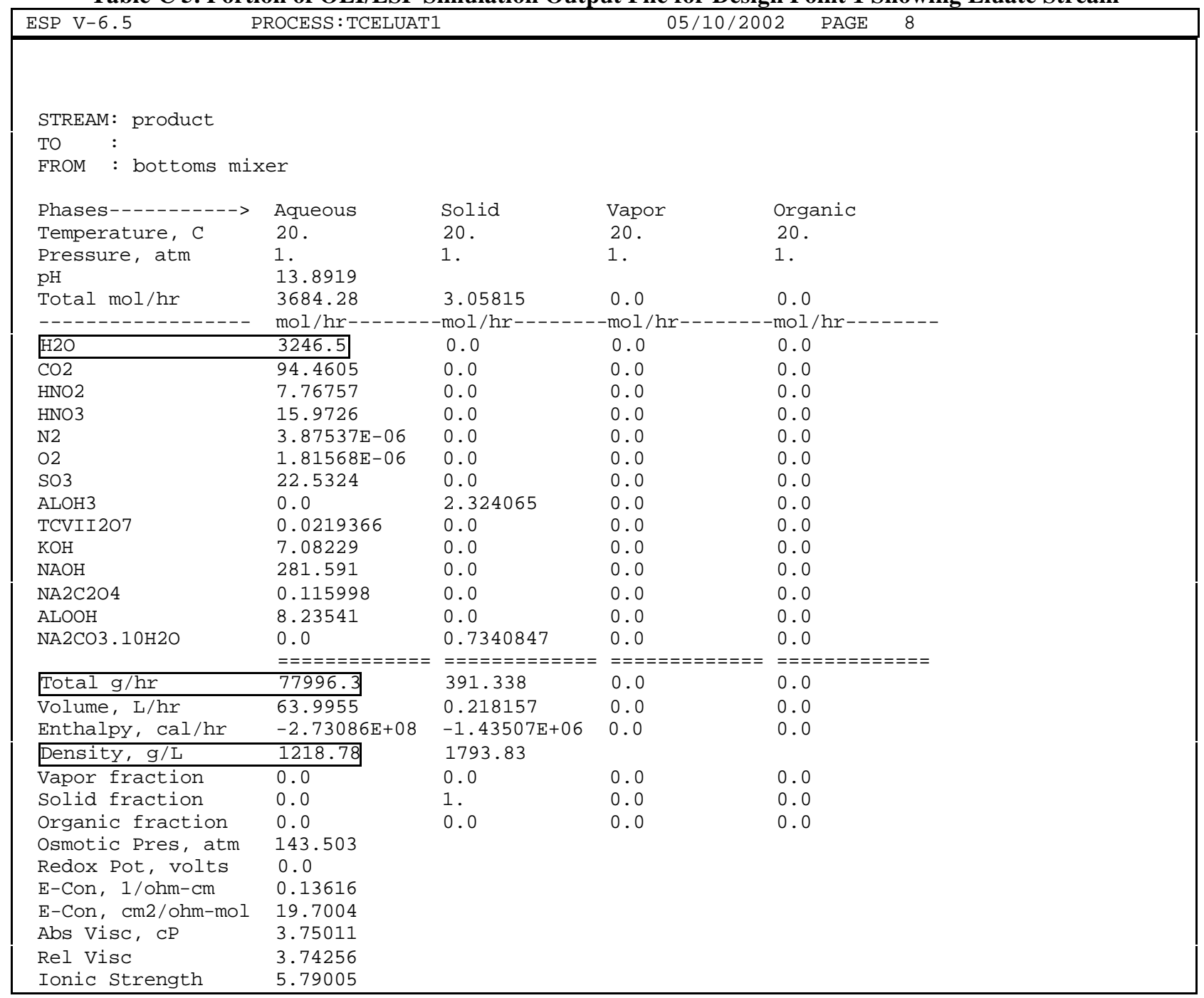


Table C-6. Portion of OLI/ESP Simulation Output File for Design Point 1 Showing Cooling (Mixer) Block

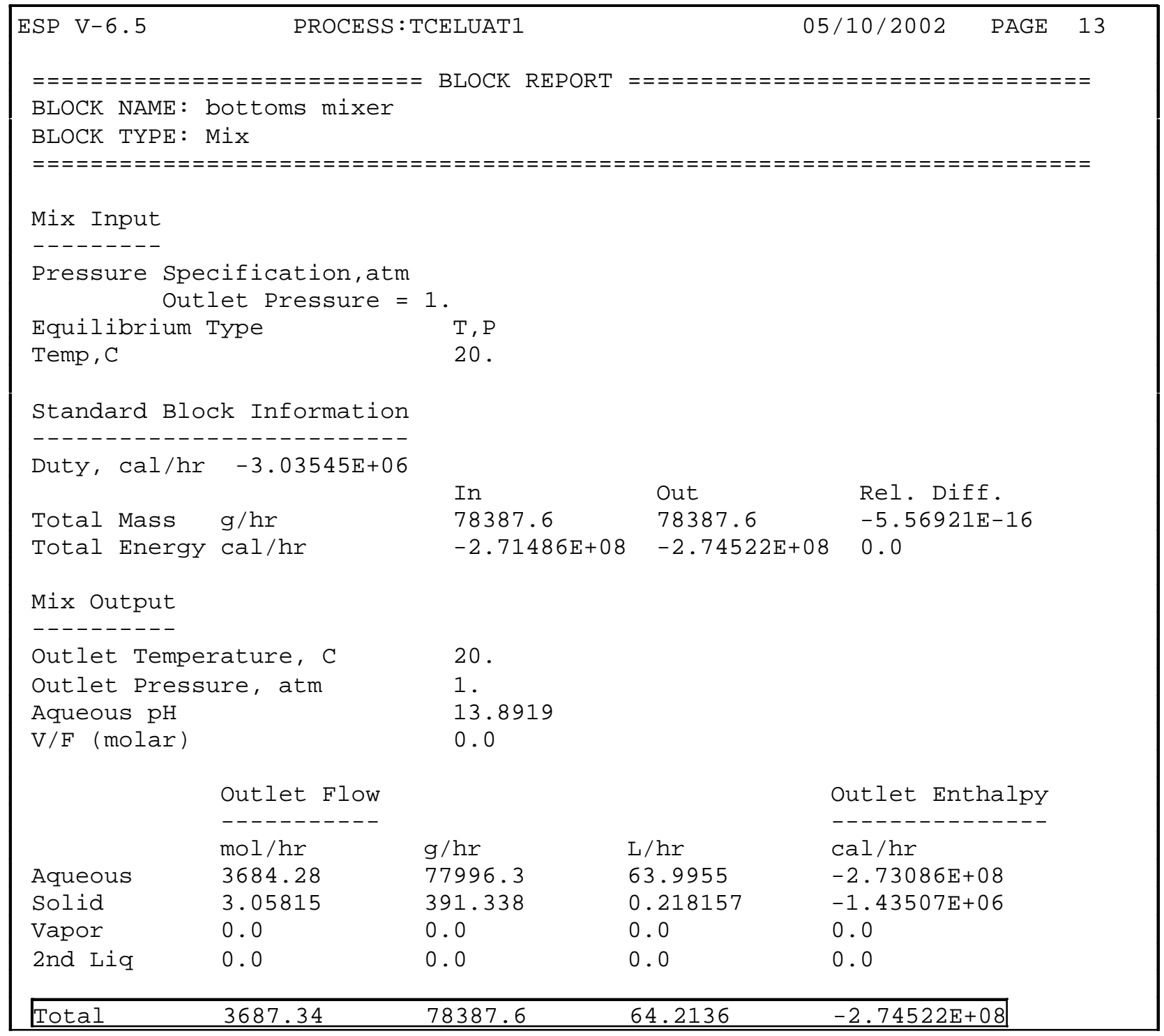




\section{Appendix D Design of Experiment}

SRT-SCS-2002-00030

May 23, 2002

To:

C. D. Barnes, 773-41A

cc:

T. B. Calloway, 999-W

S. P. Harris, 773-42A
A. S. Choi, 773-42A
D. A. Crowley, 773-43A

E. W. Holtzscheiter, 773-A

R. C. Tuckfield, 773-42A

From: $\quad$ T. B. Edwards, 773-42A (5-5148)

Statistical Consulting Section

S. P. Harris, Technical Reviewer

Date

R. C. Tuckfield, Manager

Date

Statistical Consulting Section 


\section{A Statistically Designed Test Matrix FOR A COMPUTER STUdY OF TC ElUATE SolubILITY (U)}




\subsection{INTRODUCTION}

The Statistical Consulting Section (SCS) was asked by the Process Development- Hanford River Protection Project (PD-HRPP) Group of SRTC to provide an experimental design to support the development of models for property calculations generated by the OLI/ESP software. This software is being utilized to predict information associated with the solubility of technetium (Tc) eluate for solutions and/or process conditions of interest for the Hanford RPP. The objective of this memorandum is to provide the experimental design, or test matrix, that will be used to generate the data to support this modeling effort. There are some challenging features to the design of this test matrix that must be met. These features and the approach used to meet them are described in this memorandum; statistical routines available in JMP® Version 3.2.6 [1] and Version 4.0.5 [2] (both developed by SAS Institute, Inc.) were used to support this design effort.

\subsection{Discussion}

Designing a test matrix to support the development of models for calculations made by the ESP/OLI software faces some unique challenges. These challenges are identified in this section along with the approach taken to address them.

\subsection{Factor Space of Interest}

The development of a model for each of several properties is of interest in this study. However, there is the potential for each such model to be a rather complex function of factors associated with the initial solution and/or process conditions. The first challenge to be faced in selecting this test matrix is to identify the initial solution and/or process conditions that define the factors for this study. Based upon input from the PD-HRPP group, the properties and process conditions given in Table 1 were selected to define the design or factor space of interest in the development of this test matrix.

Table 1: Initial Factor Intervals Defining the Design Space

(Chemical Species in Weight Fractions, WFs)

$\begin{array}{ccccccccccc}\text { Factor } & \mathrm{AlO}_{2} & \mathrm{C}_{2} \mathrm{O}_{4} & \mathrm{CO}_{3} & \mathrm{NO}_{2} & \mathrm{NO}_{3} & \mathrm{OH} & \mathrm{SO}_{4} & \mathrm{TcO}_{4} & \text { Temperature } \\ \text { Interval } & (\mathrm{WF}) & (\mathrm{WF}) & (\mathrm{WF}) & (\mathrm{WF}) & (\mathrm{WF}) & (\mathrm{WF}) & (\mathrm{WF}) & (\mathrm{WF}) & \left({ }^{\circ} \mathrm{C}\right) \\ \text { Min } & 0.00750 & 0.00001 & 0.16500 & 0.03900 & 0.10500 & 0.01600 & 0.00001 & 0.00042 & 20 \\ \operatorname{Max} & 0.03000 & 0.02050 & 0.80500 & 0.36000 & 0.73500 & 0.04100 & 0.19500 & 0.02150 & 70\end{array}$

Thus, there are 9 factors for which levels must be determined to define one experimental trial (or row) of the test matrix.

However, there is an additional constraint that is to be imposed on this factor space - the solution of the 8 chemical species identified in Table 1 is to be considered as a mixture (see Cornell [3]). This is the second challenging feature of this design task. Specifically, only combinations of the weight fractions of $\mathrm{AlO}_{2}$, $\mathrm{C}_{2} \mathrm{O}_{4}, \mathrm{CO}_{3}, \mathrm{NO}_{2}, \mathrm{NO}_{3}, \mathrm{OH}, \mathrm{SO}_{4}$, and $\mathrm{TcO}_{4}$ satisfying the following constraint are to be considered valid for this experimental design: 


\section{Equation (1).}

$$
\mathrm{AlO} 2+\mathrm{C}_{2} \mathrm{O}_{4}+\mathrm{CO}_{3}+\mathrm{NO}_{2}+\mathrm{NO}_{3}+\mathrm{OH}+\mathrm{SO}_{4}+\mathrm{TcO}_{4}=1
$$

The temperature factor is considered a "process" factor for these experiments. The mixture constraint and the presence of a process factor do impact the models of interest. This is discussed in the next section.

\subsection{Mathematical Models of Interest}

This experimental design is needed to support the development of mathematical models for properties of interest for which values are generated by the ESP/OLI software. Models of interest for this effort range from simple linear models to the more complex, response surface models. However, the forms of these models are restricted due to the mixture aspects of the problem and the presence of a process factor. A discussion of the impact of these features is provided by Cornell [3].

For this Tc eluate solubility study, the most complex model that will be considered as part of this design effort is of the form given by equation (2): 


\section{Equation (2).}

$$
\begin{aligned}
& \text { Response }_{\text {ESP/OLI }}=\beta_{1} \mathrm{AlO}_{2}+\beta_{2} \mathrm{C}_{2} \mathrm{O}_{4}+\beta_{3} \mathrm{AlO}_{2} \cdot \mathrm{C}_{2} \mathrm{O}_{4}+\beta_{4} \mathrm{CO}_{3}+\beta_{5} \mathrm{AlO}_{2} \cdot \mathrm{CO}_{3} \\
& +\beta_{6} \mathrm{C}_{2} \mathrm{O}_{4} \cdot \mathrm{CO}_{3}+\beta_{7} \mathrm{NO}_{2}+\beta_{8} \mathrm{AlO}_{2} \cdot \mathrm{NO}_{2}+\beta_{9} \mathrm{C}_{2} \mathrm{O}_{4} \cdot \mathrm{NO}_{2} \\
& +\beta_{10} \mathrm{CO}_{3} \cdot \mathrm{NO}_{2}+\beta_{11} \mathrm{NO}_{3}+\beta_{12} \mathrm{AlO}_{2} \cdot \mathrm{NO}_{3}+\beta_{13} \mathrm{C}_{2} \mathrm{O}_{4} \cdot \mathrm{NO}_{3} \\
& +\beta_{14} \mathrm{CO}_{3} \cdot \mathrm{NO}_{3}+\beta_{15} \mathrm{NO}_{2} \cdot \mathrm{NO}_{3}+\beta_{16} \mathrm{OH}+\beta_{17} \mathrm{AlO}{ }_{2} \cdot \mathrm{OH} \\
& +\beta_{18} \mathrm{C}_{2} \mathrm{O}_{4} \cdot \mathrm{OH}+\beta_{19} \mathrm{CO}_{3} \cdot \mathrm{OH}+\beta_{20} \mathrm{NO}_{2} \cdot \mathrm{OH}+\beta_{21} \mathrm{NO}_{3} \cdot \mathrm{OH} \\
& +\beta_{22} \mathrm{SO}_{4}+\beta_{23} \mathrm{AlO}_{2} \cdot \mathrm{SO}_{4}+\beta_{24} \mathrm{C}_{2} \mathrm{O}_{4} \cdot \mathrm{SO}_{4}+\beta_{25} \mathrm{CO}_{3} \cdot \mathrm{SO}_{4} \\
& +\beta_{26} \mathrm{NO}_{2} \cdot \mathrm{SO}_{4}+\beta_{27} \mathrm{NO}_{3} \cdot \mathrm{SO}_{4}+\beta_{28} \mathrm{OH} \cdot \mathrm{SO}_{4} \\
& +\beta_{29} \mathrm{TcO}_{4}+\beta_{30} \mathrm{AlO}_{2} \cdot \mathrm{TcO}_{4}+\beta_{31} \mathrm{C}_{2} \mathrm{O}_{4} \cdot \mathrm{TcO}_{4} \\
& +\beta_{32} \mathrm{CO}_{3} \cdot \mathrm{TcO}_{4}+\beta_{33} \mathrm{NO}_{2} \cdot \mathrm{TcO}_{4}+\beta_{34} \mathrm{NO}_{3} \cdot \mathrm{TcO}_{4} \\
& +\beta_{35} \mathrm{OH} \cdot \mathrm{TcO}_{4}+\beta_{36} \mathrm{SO}_{4} \cdot \mathrm{TcO}_{4}+\beta_{37} \mathrm{AlO}_{2} \cdot \mathrm{Temp}+\beta_{38} \mathrm{C}_{2} \mathrm{O}_{4} \cdot \mathrm{Temp} \\
& +\beta_{39} \mathrm{AlO}_{2} \cdot \mathrm{C}_{2} \mathrm{O}_{4} \cdot \mathrm{Temp}+\beta_{40} \mathrm{CO}_{3} \cdot \text { Temp }+\beta_{41} \mathrm{AlO}_{2} \cdot \mathrm{CO}_{3} \cdot \text { Temp } \\
& +\beta_{42} \mathrm{C}_{2} \mathrm{O}_{4} \cdot \mathrm{CO}_{3} \cdot \mathrm{Temp}+\beta_{43} \mathrm{NO}_{2} \cdot \mathrm{Temp}+\beta_{44} \mathrm{AlO}_{2} \cdot \mathrm{NO}_{2} \cdot \text { Temp } \\
& +\beta_{45} \mathrm{C}_{2} \mathrm{O}_{4} \cdot \mathrm{NO}_{2} \cdot \text { Temp }+\beta_{46} \mathrm{CO}_{3} \cdot \mathrm{NO}_{2} \cdot \text { Temp }+\beta_{47} \mathrm{NO}_{3} \cdot \text { Temp } \\
& +\beta_{48} \mathrm{AlO}_{2} \cdot \mathrm{NO}_{3} \cdot \mathrm{Temp}+\beta_{49} \mathrm{C}_{2} \mathrm{O}_{4} \cdot \mathrm{NO}_{3} \cdot \mathrm{Temp}+\beta_{50} \mathrm{CO}_{3} \cdot \mathrm{NO}_{3} \cdot \mathrm{Temp} \\
& +\beta_{51} \mathrm{NO}_{2} \cdot \mathrm{NO}_{3} \cdot \mathrm{Temp}+\beta_{52} \mathrm{OH} \cdot \mathrm{Temp}+\beta_{53} \mathrm{AlO}_{2} \cdot \mathrm{OH} \cdot \mathrm{Temp} \\
& +\beta_{54} \mathrm{C}_{2} \mathrm{O}_{4} \cdot \mathrm{OH} \cdot \mathrm{Temp}+\beta_{55} \mathrm{CO}_{3} \cdot \mathrm{OH} \cdot \mathrm{Temp}+\beta_{56} \mathrm{NO}_{2} \cdot \mathrm{OH} \cdot \mathrm{Temp} \\
& +\beta_{57} \mathrm{NO}_{3} \cdot \mathrm{OH} \cdot \mathrm{Temp}+\beta_{58} \mathrm{SO}_{4} \cdot \mathrm{Temp}+\beta_{59} \mathrm{AlO}_{2} \cdot \mathrm{SO}_{4} \cdot \mathrm{Temp} \\
& +\beta_{60} \mathrm{C}_{2} \mathrm{O}_{4} \cdot \mathrm{SO}_{4} \cdot \mathrm{Temp}+\beta_{61} \mathrm{CO}_{3} \cdot \mathrm{SO}_{4} \cdot \mathrm{Temp}+\beta_{62} \mathrm{NO}_{2} \cdot \mathrm{SO}_{4} \cdot \mathrm{Temp} \\
& +\beta_{63} \mathrm{NO}_{3} \cdot \mathrm{SO}_{4} \cdot \mathrm{Temp}+\beta_{64} \mathrm{OH} \cdot \mathrm{SO}_{4} \cdot \mathrm{Temp}+\beta_{65} \mathrm{TcO}_{4} \cdot \mathrm{Temp} \\
& +\beta_{66} \mathrm{AlO}_{2} \cdot \mathrm{TcO}_{4} \cdot \mathrm{Temp}+\beta_{67} \mathrm{C}_{2} \mathrm{O}_{4} \cdot \mathrm{TcO}_{4} \cdot \mathrm{Temp}+\beta_{68} \mathrm{CO}_{3} \cdot \mathrm{TcO}_{4} \cdot \mathrm{Temp} \\
& +\beta_{69} \mathrm{NO}_{2} \cdot \mathrm{TcO}_{4} \cdot \mathrm{Temp}+\beta_{70} \mathrm{NO}_{3} \cdot \mathrm{TcO}_{4} \cdot \mathrm{Temp}+\beta_{71} \mathrm{OH} \cdot \mathrm{TcO}_{4} \cdot \mathrm{Temp} \\
& +\beta_{72} \mathrm{SO}_{4} \cdot \mathrm{TcO}_{4} \cdot \mathrm{Temp}
\end{aligned}
$$

where the $\beta$ 's are unknown parameters that may or may not be significant in defining the function. Note that the impacts of the mixture variables and process variables are evident in equation (2) in that there is no intercept term and "Temp" (representing the temperature factor) only appears in the model in cross terms with the mixture variables. Also, note that the minimum number of design points required to fit this model is 72 .

\subsection{Preliminary Design Points}

Classical, well-known statistical designs are available for generating data for fitting general response surface models. Methods are also available to help if mixture response surface models are of interest. JMP 
Version 4.0.5 provides a "Custom Design" feature under its "Design of Experiment (DOE)" platform that supports such a design effort. This feature was used to generate an initial set of 36 design points, which are provided in Table 2. These points were selected using JMP's coordinate exchange algorithm and are optimal for a model consisting of the first 36 terms of equation (2). This first set of design points addresses the mixture aspects of equation (2) but not the process variable. The process variable (Temp) is handled by conducting the 36 mixture experiments both at the low and at the high temperature extremes (i.e., 20 and 70 $\left.{ }^{\circ} \mathrm{C}\right)$. This provides the 72 experimental points that are required to complete the design to support the model given by equation (2).

Table 2: JMP Design Points for Mixture Response Surface Model (Values are given as weight fractions, WFs)

\begin{tabular}{|c|c|c|c|c|c|c|c|}
\hline $\mathrm{AlO}_{2}$ & $\mathrm{C}_{2} \mathrm{O}_{4}$ & $\mathrm{CO}_{3}$ & $\mathrm{NO}_{2}$ & $\mathrm{NO}_{3}$ & $\mathrm{OH}$ & $\mathrm{SO}_{4}$ & $\mathrm{TcO}_{4}$ \\
\hline (WF) & (WF) & (WF) & (WF) & (WF) & (WF) & (WF) & (WF) \\
\hline .030000 & 0.020500 & 0.386845 & 0.039000 & 0.482225 & 0.041000 & 0.000010 & 0.000420 \\
\hline 0.017228 & 0.015282 & 0.615978 & 0.074078 & 0.233978 & 0.018978 & 0.002988 & 0.021490 \\
\hline 0.029990 & 0.020018 & 0.566959 & 0.121159 & 0.185959 & 0.036459 & 0.017969 & 0.021490 \\
\hline 0.029990 & 0.020490 & 0.453658 & 0.200058 & 0.200658 & 0.040990 & 0.032668 & 0.021490 \\
\hline 0.030000 & 0.000010 & 0.490323 & 0.233127 & 0.157839 & 0.016000 & 0.072282 & 0.000420 \\
\hline 0.029990 & 0.020500 & 0.746713 & 0.073667 & 0.107567 & 0.016000 & 0.005143 & 0.000420 \\
\hline 0.007500 & 0.020500 & 0.303183 & 0.360000 & 0.112686 & 0.041000 & 0.154711 & 0.000420 \\
\hline 0.029990 & 0.020490 & 0.390883 & 0.136883 & 0.202883 & 0.040990 & 0.156390 & 0.021490 \\
\hline 0.007500 & 0.020500 & 0.631250 & 0.174450 & 0.112770 & 0.024010 & 0.029100 & 0.000420 \\
\hline 0.029990 & 0.020490 & 0.358783 & 0.233183 & 0.170783 & 0.040990 & 0.124290 & 0.021490 \\
\hline 0.007500 & 0.000010 & 0.571187 & 0.104883 & 0.105000 & 0.016000 & 0.195000 & 0.000420 \\
\hline 0.007500 & 0.020500 & 0.786570 & 0.039000 & 0.105000 & 0.041000 & 0.000010 & 0.000420 \\
\hline 0.007500 & 0.020500 & 0.472619 & 0.181313 & 0.253825 & 0.041000 & 0.001745 & 0.021500 \\
\hline 0.029990 & 0.020490 & 0.286483 & 0.128583 & 0.414483 & 0.040990 & 0.057493 & 0.021490 \\
\hline 0.019980 & 0.000020 & 0.735480 & 0.065580 & 0.162480 & 0.016010 & 0.000020 & 0.000430 \\
\hline 0.029990 & 0.020490 & 0.277708 & 0.215908 & 0.280708 & 0.040990 & 0.112718 & 0.021490 \\
\hline 0.030000 & 0.020500 & 0.510301 & 0.109592 & 0.300392 & 0.016000 & 0.012795 & 0.000420 \\
\hline 0.030000 & 0.000010 & 0.541318 & 0.144902 & 0.208074 & 0.041000 & 0.013197 & 0.021500 \\
\hline 0.029990 & 0.020490 & 0.295333 & 0.169533 & 0.297333 & 0.040990 & 0.145910 & 0.000420 \\
\hline
\end{tabular}




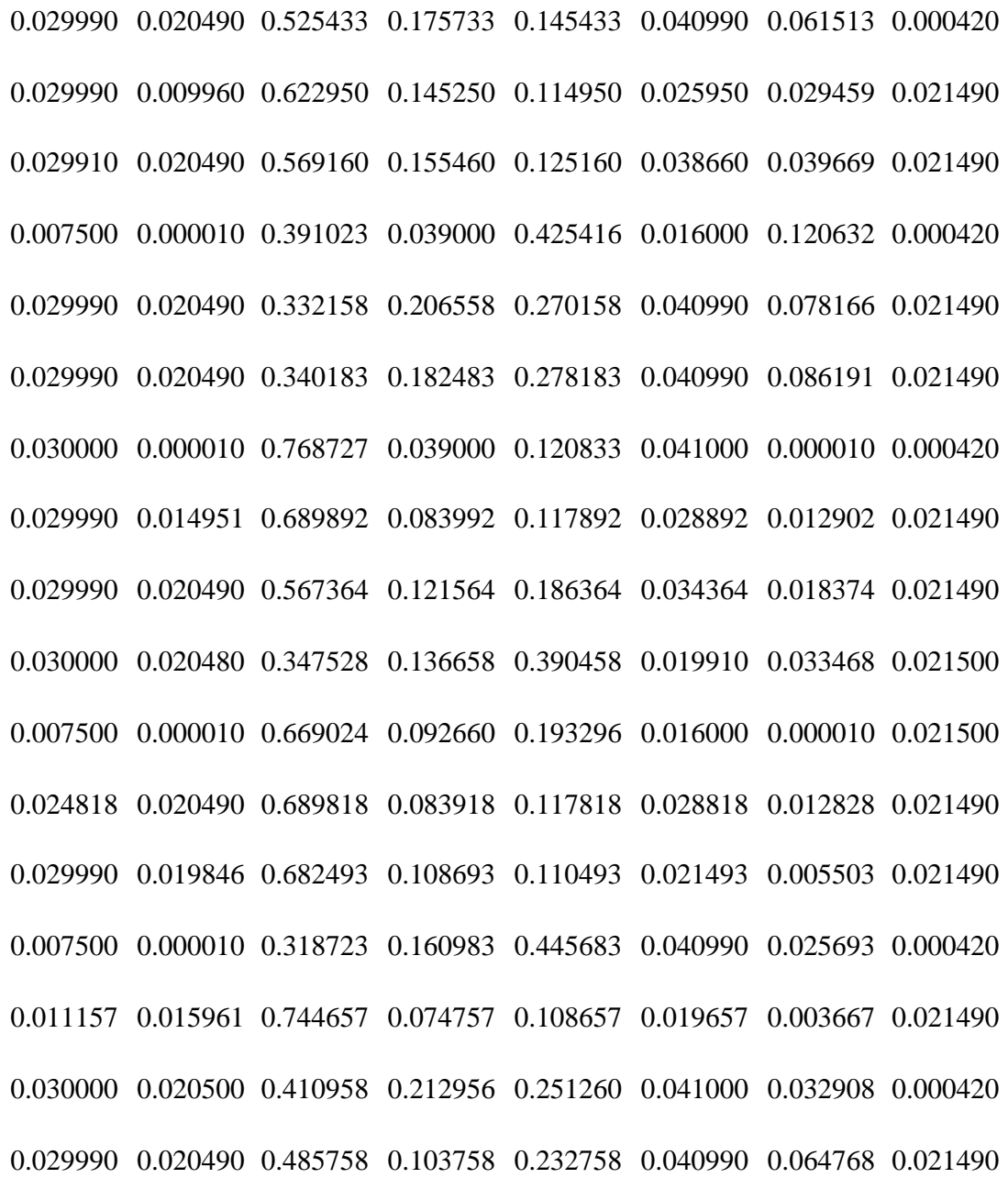

For a classical experimental setting, this set of 72 design points would go a long way toward the final test matrix. However, the setting for this statistical design is not a classical experimental setting; it is, in fact, computer experimentation. There is no experimental error associated with the outcome generated from a design point in this setting (i.e., for a fixed set of inputs the outcome from the computer [experimental] run is deterministic). This is an additional challenging feature of this design task.

\subsection{Designs for Computer Experimentation}

The statistical perspective of design problems involving computer experimentation has been explored [4][7]. These references identify and discuss the unique aspects of this design and analysis problem. A method for generating orthogonal Latin hypercubes (OLHs) and their advantages for such problems are presented in [7]. An advantage of a Latin Hypercube approach is that it facilitates each of the input variables having all portions of its range represented [7]. Thus, the approach provides a "space-filling" (for a factor space such as that defined by Table 1) set of design points. Also, orthogonal Latin hypercube designs "guarantee that the estimates of quadratic effects and bilinear interaction effects are uncorrelated with estimates of linear effects. However, the estimates of quadratic and bilinear interaction effects are correlated with each other." [7] 
From [7], an OLH consisting of $\mathrm{n}$ rows can be constructed when $\mathrm{n}$ is a power of 2 or a power of 2 plus 1 (i.e., $2^{\mathrm{m}}$ or $2^{\mathrm{m}}+1$ ). A method is provided in [7] for constructing such an OLH with $2 \mathrm{~m}-2$ columns. The value of 2m-2 must be equal to or greater than the number of factors of interest. A value of 5 will be used for $\mathrm{m}$ (with $2 \mathrm{~m}-2=8$, which allows 8 factors to be investigated). A value of $\mathrm{m}=5$ leads to a value for $\mathrm{n}$ of 32 or 33 . For this design, a value of 33 will be selected for $n$. Thus, the interval of possible values for each input is divided into 33 equal sub-intervals with representative values for each sub-interval as given in Table 3. 
Table 3: Factor Sub-Intervals in Weight Fractions

(WFs) Defining the Design Space

\begin{tabular}{|c|c|c|c|c|c|c|c|c|c|}
\hline Coded & $\mathrm{AlO}_{2}$ & $\mathrm{C}_{2} \mathrm{O}_{4}$ & $\mathrm{CO}_{3}$ & $\mathrm{NO}_{2}$ & $\mathrm{NO}_{3}$ & $\mathrm{OH}$ & $\mathrm{SO}_{4}$ & $\mathrm{TcO}_{4}$ & Temperature \\
\hline Values & (WF) & $(\mathrm{WF})$ & $(\mathrm{WF})$ & (WF) & (WF) & $(\mathrm{WF})$ & (WF) & (WF) & $\left({ }^{\circ} \mathrm{C}\right)$ \\
\hline-16 & 0.00750 & 0.00001 & 0.16500 & 0.03900 & 0.10500 & 0.01600 & 0.00001 & 0.00042 & 20.0000 \\
\hline-15 & 0.00820 & 0.00065 & 0.18500 & 0.04903 & 0.12469 & 0.01678 & 0.00610 & 0.00107 & 21.5625 \\
\hline-14 & 0.00891 & 0.00129 & 0.20500 & 0.05906 & 0.14438 & 0.01756 & 0.01220 & 0.00173 & 23.1250 \\
\hline-13 & 0.00961 & 0.00193 & 0.22500 & 0.06909 & 0.16406 & 0.01834 & 0.01829 & 0.00239 & 24.6875 \\
\hline-12 & 0.01031 & 0.00257 & 0.24500 & 0.07913 & 0.18375 & 0.01913 & 0.02438 & 0.00305 & 26.2500 \\
\hline-11 & 0.01102 & 0.00321 & 0.26500 & 0.08916 & 0.20344 & 0.01991 & 0.03048 & 0.00371 & 27.8125 \\
\hline-10 & 0.01172 & 0.00385 & 0.28500 & 0.09919 & 0.22313 & 0.02069 & 0.03657 & 0.00437 & 29.3750 \\
\hline-9 & 0.01242 & 0.00449 & 0.30500 & 0.10922 & 0.24281 & 0.02147 & 0.04266 & 0.00503 & 30.9375 \\
\hline-8 & 0.01313 & 0.00513 & 0.32500 & 0.11925 & 0.26250 & 0.02225 & 0.04876 & 0.00569 & 32.5000 \\
\hline-7 & 0.01383 & 0.00577 & 0.34500 & 0.12928 & 0.28219 & 0.02303 & 0.05485 & 0.00635 & 34.0625 \\
\hline-6 & 0.01453 & 0.00641 & 0.36500 & 0.13931 & 0.30188 & 0.02381 & 0.06094 & 0.00700 & 35.6250 \\
\hline-5 & 0.01523 & 0.00705 & 0.38500 & 0.14934 & 0.32156 & 0.02459 & 0.06704 & 0.00766 & 37.1875 \\
\hline-4 & 0.01594 & 0.00769 & 0.40500 & 0.15938 & 0.34125 & 0.02538 & 0.07313 & 0.00832 & 38.7500 \\
\hline-3 & 0.01664 & 0.00833 & 0.42500 & 0.16941 & 0.36094 & 0.02616 & 0.07922 & 0.00898 & 40.3125 \\
\hline-2 & 0.01734 & 0.00897 & 0.44500 & 0.17944 & 0.38063 & 0.02694 & 0.08532 & 0.00964 & 41.8750 \\
\hline-1 & 0.01805 & 0.00961 & 0.46500 & 0.18947 & 0.40031 & 0.02772 & 0.09141 & 0.01030 & 43.4375 \\
\hline 0 & 0.01875 & 0.01026 & 0.48500 & 0.19950 & 0.42000 & 0.02850 & 0.09751 & 0.01096 & 45.0000 \\
\hline 1 & 0.01945 & 0.01090 & 0.50500 & 0.20953 & 0.43969 & 0.02928 & 0.10360 & 0.01162 & 46.5625 \\
\hline 2 & 0.02016 & 0.01154 & 0.52500 & 0.21956 & 0.45938 & 0.03006 & 0.10969 & 0.01228 & 48.1250 \\
\hline 3 & 0.02086 & 0.01218 & 0.54500 & 0.22959 & 0.47906 & 0.03084 & 0.11579 & 0.01293 & 49.6875 \\
\hline 4 & 0.02156 & 0.01282 & 0.56500 & 0.23963 & 0.49875 & 0.03163 & 0.12188 & 0.01359 & 51.2500 \\
\hline 5 & 0.02227 & 0.01346 & 0.58500 & 0.24966 & 0.51844 & 0.03241 & 0.12797 & 0.01425 & 52.8125 \\
\hline 6 & 0.02297 & 0.01410 & 0.60500 & 0.25969 & 0.53813 & 0.03319 & 0.13407 & 0.01491 & 54.3750 \\
\hline
\end{tabular}




$\begin{array}{ccccccccccc}7 & 0.02367 & 0.01474 & 0.62500 & 0.26972 & 0.55781 & 0.03397 & 0.14016 & 0.01557 & 55.9375 \\ 8 & 0.02438 & 0.01538 & 0.64500 & 0.27975 & 0.57750 & 0.03475 & 0.14625 & 0.01623 & 57.5000 \\ 9 & 0.02508 & 0.01602 & 0.66500 & 0.28978 & 0.59719 & 0.03553 & 0.15235 & 0.01689 & 59.0625 \\ 10 & 0.02578 & 0.01666 & 0.68500 & 0.29981 & 0.61688 & 0.03631 & 0.15844 & 0.01755 & 60.6250 \\ 11 & 0.02648 & 0.01730 & 0.70500 & 0.30984 & 0.63656 & 0.03709 & 0.16453 & 0.01821 & 62.1875 \\ 12 & 0.02719 & 0.01794 & 0.72500 & 0.31988 & 0.65625 & 0.03788 & 0.17063 & 0.01886 & 63.7500 \\ 13 & 0.02789 & 0.01858 & 0.74500 & 0.32991 & 0.67594 & 0.03866 & 0.17672 & 0.01952 & 65.3125 \\ 14 & 0.02859 & 0.01922 & 0.76500 & 0.33994 & 0.69563 & 0.03944 & 0.18281 & 0.02018 & 66.8750 \\ 15 & 0.02930 & 0.01986 & 0.78500 & 0.34997 & 0.71531 & 0.04022 & 0.18891 & 0.02084 & 68.4375 \\ 16 & 0.03000 & 0.02050 & 0.80500 & 0.36000 & 0.73500 & 0.04100 & 0.19500 & 0.02150 & 70.0000\end{array}$

However, the method espoused in [7] cannot be used directly for this design problem due to the mixture constraint on factors: $\mathrm{AlO} 2, \mathrm{C}_{2} \mathrm{O}_{4}, \mathrm{CO}_{3}, \mathrm{NO}_{2}, \mathrm{NO}_{3}, \mathrm{OH}, \mathrm{SO}_{4}$, and $\mathrm{TcO}_{4}$. The method may be used on 7 of these factors (say all of the factors except $\mathrm{CO}_{3}$ ) and temperature, but then the $\mathrm{CO}_{3}$ level would need to be forced to equal 1 minus the levels of the other 7 mixture factors. Such an approach was used. Using the method outlined in [7] and working with the coded intervals of Table 3 for [AlO2], $\mathrm{C}_{2} \mathrm{O}_{4}, \mathrm{NO}_{2}, \mathrm{NO}_{3}, \mathrm{OH}$, $\mathrm{SO}_{4}, \mathrm{TcO}_{4}$, and temperature lead to the selection of a orthogonal Latin hypercube for this problem expressed in the coded intervals given by Table 4 . Note that only 8 columns were generated by the method of [7] and these 8 input columns are given in Table 4 along with a shaded and blank, placeholder column for $\mathrm{CO}_{3}$. It is easily verified that the input columns presented in Table 4 are orthogonal (i.e., any two input columns $\mathbf{u}$ and $\mathbf{v}$ from Table 4 satisfy $\mathbf{u}^{\mathrm{T}} \mathbf{v}=0$ ).

Table 4: Initial Coded Orthogonal Latin Hypercube Design

$\begin{array}{cccccccccc}\text { Initial } & \mathrm{AlO}_{2} & \mathrm{C}_{2} \mathrm{O}_{4} & \mathrm{CO}_{3} & \mathrm{NO}_{2} & \mathrm{NO}_{3} & \mathrm{OH} & \mathrm{SO}_{4} & \mathrm{TcO}_{4} & \text { Temperature } \\ \text { Design Pt } & \text { coded } & \text { coded } & & \text { coded } & \text { coded } & \text { coded } & \text { coded } & \text { coded } & \text { coded } \\ 1 & 1 & -2 & -4 & -8 & -16 & 15 & 13 & 9 \\ 2 & 2 & 1 & -3 & -7 & -15 & -16 & -14 & -10 \\ 3 & 3 & -4 & 2 & -6 & -14 & -13 & 15 & 11 \\ 4 & 4 & 3 & 1 & -5 & -13 & 14 & -16 & -12 \\ 5 & 5 & -6 & -8 & 4 & -12 & 11 & -9 & 13\end{array}$




\begin{tabular}{|c|c|c|c|c|c|c|c|c|}
\hline 6 & 6 & 5 & -7 & 3 & -11 & -12 & 10 & -14 \\
\hline 7 & 7 & -8 & 6 & 2 & -10 & -9 & -11 & 15 \\
\hline 8 & 8 & 7 & 5 & 1 & -9 & 10 & 12 & -16 \\
\hline 9 & 9 & -10 & -12 & -16 & 8 & 7 & 5 & -1 \\
\hline 10 & 10 & 9 & -11 & -15 & 7 & -8 & -6 & 2 \\
\hline 11 & 11 & -12 & 10 & -14 & 6 & -5 & 7 & -3 \\
\hline 12 & 12 & 11 & 9 & -13 & 5 & 6 & -8 & 4 \\
\hline 13 & 13 & -14 & -16 & 12 & 4 & 3 & -1 & -5 \\
\hline 14 & 14 & 13 & -15 & 11 & 3 & -4 & 2 & 6 \\
\hline 15 & 15 & -16 & 14 & 10 & 2 & -1 & -3 & -7 \\
\hline 16 & 16 & 15 & 13 & 9 & 1 & 2 & 4 & 8 \\
\hline 17 & 0 & 0 & 0 & 0 & 0 & 0 & 0 & 0 \\
\hline 18 & -1 & 2 & 4 & 8 & 16 & -15 & -13 & -9 \\
\hline 19 & -2 & -1 & 3 & 7 & 15 & 16 & 14 & 10 \\
\hline 20 & -3 & 4 & -2 & 6 & 14 & 13 & -15 & -11 \\
\hline 21 & -4 & -3 & -1 & 5 & 13 & -14 & 16 & 12 \\
\hline 22 & -5 & 6 & 8 & -4 & 12 & -11 & 9 & -13 \\
\hline 23 & -6 & -5 & 7 & -3 & 11 & 12 & -10 & 14 \\
\hline 24 & -7 & 8 & -6 & -2 & 10 & 9 & 11 & -15 \\
\hline 25 & -8 & -7 & -5 & -1 & 9 & -10 & -12 & 16 \\
\hline 26 & -9 & 10 & 12 & 16 & -8 & -7 & -5 & 1 \\
\hline 27 & -10 & -9 & 11 & 15 & -7 & 8 & 6 & -2 \\
\hline 28 & -11 & 12 & -10 & 14 & -6 & 5 & -7 & 3 \\
\hline 29 & -12 & -11 & -9 & 13 & -5 & -6 & 8 & -4 \\
\hline 30 & -13 & 14 & 16 & -12 & -4 & -3 & 1 & 5 \\
\hline 31 & -14 & -13 & 15 & -11 & -3 & 4 & -2 & -6 \\
\hline 32 & -15 & 16 & -14 & -10 & -2 & 1 & 3 & 7 \\
\hline
\end{tabular}


Translating the coded information of Table 4 into the original units for the inputs and computing the value of $\mathrm{CO}_{3}$ so that the mixture constraint is satisfied lead to Table 5, the initial set of design points derived using the OLH approach. Note that five of the values calculated for $\mathrm{CO}_{3}$ are less than zero (i.e., an invalid value). Such values are shaded in Table 5. Also, recall that Table 1 restricts the design space for this problem, and note that the restriction was not imposed during the determination of the $\mathrm{CO}_{3}$ values (only the sum to 1 constraint was imposed). A review of the $\mathrm{CO}_{3}$ values finds that nine initial design points of Table 5 are outside the region of interest for $\mathrm{CO}_{3}$. These points do not satisfy the restrictions of Table 1 and are also shaded in Table 5. 
Table 5: Initial Orthogonal Latin Hypercube Design with Chemical Species in Weight Fractions (WFs)

\begin{tabular}{|c|c|c|c|c|c|c|c|c|c|}
\hline Initial & $\mathrm{AlO}_{2}$ & $\mathrm{C}_{2} \mathrm{O}_{4}$ & $\mathrm{CO}_{3}$ & $\mathrm{NO}_{2}$ & $\mathrm{NO}_{3}$ & $\mathrm{OH}$ & $\mathrm{SO}_{4}$ & $\mathrm{TcO}_{4}$ & Temperature \\
\hline Design Pt & (WF) & (WF) & (WF) & (WF) & (WF) & (WF) & (WF) & (WF) & $\left({ }^{\circ} \mathrm{C}\right)$ \\
\hline 1 & 0.019453 & 0.008974 & 0.325267 & 0.159375 & 0.262500 & 0.016000 & 0.188907 & 0.019524 & 59.0625 \\
\hline 2 & 0.020156 & 0.010895 & 0.498826 & 0.169406 & 0.282188 & 0.016781 & 0.000010 & 0.001738 & 29.3750 \\
\hline 3 & 0.020859 & 0.007694 & 0.393315 & 0.219563 & 0.301875 & 0.017563 & 0.018290 & 0.020841 & 62.1875 \\
\hline 4 & 0.021563 & 0.012176 & 0.233591 & 0.209531 & 0.321563 & 0.018344 & 0.182813 & 0.000420 & 26.2500 \\
\hline 5 & 0.022266 & 0.006413 & 0.164632 & 0.119250 & 0.498750 & 0.019125 & 0.164533 & 0.005031 & 65.3125 \\
\hline 6 & 0.022969 & 0.013457 & 0.293393 & 0.129281 & 0.479063 & 0.019906 & 0.024384 & 0.017548 & 23.1250 \\
\hline 7 & 0.023672 & 0.005133 & 0.185068 & 0.259688 & 0.459375 & 0.020688 & 0.042664 & 0.003714 & 68.4375 \\
\hline 8 & 0.024375 & 0.014737 & 0.072771 & 0.249656 & 0.439688 & 0.021469 & 0.158439 & 0.018865 & 20.0000 \\
\hline 9 & 0.025078 & 0.003852 & 0.597782 & 0.079125 & 0.105000 & 0.034750 & 0.140159 & 0.014254 & 43.4375 \\
\hline 10 & 0.025781 & 0.016018 & 0.654623 & 0.089156 & 0.124688 & 0.033969 & 0.048758 & 0.007008 & 48.1250 \\
\hline 11 & 0.026484 & 0.002571 & 0.410960 & 0.299813 & 0.144375 & 0.033188 & 0.067038 & 0.015571 & 40.3125 \\
\hline 12 & 0.027188 & 0.017298 & 0.329508 & 0.289781 & 0.164063 & 0.032406 & 0.134066 & 0.005690 & 51.2500 \\
\hline 13 & 0.027891 & 0.001291 & 0.117857 & 0.039000 & 0.656250 & 0.031625 & 0.115785 & 0.010301 & 37.1875 \\
\hline 14 & 0.028594 & 0.018579 & 0.150981 & 0.049031 & 0.636563 & 0.030844 & 0.073131 & 0.012278 & 54.3750 \\
\hline 15 & 0.029297 & 0.000010 & -0.116577 & 0.339938 & 0.616875 & 0.030063 & 0.091412 & 0.008984 & 34.0625 \\
\hline 16 & 0.030000 & 0.019860 & -0.129522 & 0.329906 & 0.597188 & 0.029281 & 0.109692 & 0.013595 & 57.5000 \\
\hline 17 & 0.018750 & 0.010255 & 0.214530 & 0.199500 & 0.420000 & 0.028500 & 0.097505 & 0.010960 & 45.0000 \\
\hline 18 & 0.018047 & 0.011536 & 0.103793 & 0.239625 & 0.577500 & 0.041000 & 0.006103 & 0.002396 & 30.9375 \\
\hline 19 & 0.017344 & 0.009615 & -0.069766 & 0.229594 & 0.557813 & 0.040219 & 0.195000 & 0.020183 & 60.6250 \\
\hline 20 & 0.016641 & 0.012816 & 0.035745 & 0.179438 & 0.538125 & 0.039438 & 0.176720 & 0.001079 & 27.8125 \\
\hline 21 & 0.015938 & 0.008334 & 0.195469 & 0.189469 & 0.518438 & 0.038656 & 0.012197 & 0.021500 & 63.7500 \\
\hline 22 & 0.015234 & 0.014097 & 0.264428 & 0.279750 & 0.341250 & 0.037875 & 0.030477 & 0.016889 & 24.6875 \\
\hline 23 & 0.014531 & 0.007053 & 0.135667 & 0.269719 & 0.360938 & 0.037094 & 0.170626 & 0.004373 & 66.8750 \\
\hline
\end{tabular}




$\begin{array}{rrrrrrrrrrrrr}24 & 0.013828 & 0.015378 & 0.243992 & 0.139313 & 0.380625 & 0.036313 & 0.152346 & 0.018206 & 21.5625 \\ 25 & 0.013125 & 0.005773 & 0.356289 & 0.149344 & 0.400313 & 0.035531 & 0.036571 & 0.003055 & 70.0000 \\ 26 & 0.012422 & 0.016658 & -0.168722 & 0.319875 & 0.735000 & 0.022250 & 0.054851 & 0.007666 & 46.5625 \\ 27 & 0.011719 & 0.004492 & -0.225563 & 0.309844 & 0.715313 & 0.023031 & 0.146253 & 0.014913 & 41.8750 \\ 28 & 0.011016 & 0.017939 & 0.018100 & 0.099188 & 0.695625 & 0.023813 & 0.127972 & 0.006349 & 49.6875 \\ 29 & 0.010313 & 0.003212 & 0.099552 & 0.109219 & 0.675938 & 0.024594 & 0.060944 & 0.016230 & 38.7500 \\ 30 & 0.009609 & 0.019219 & 0.311203 & 0.360000 & 0.183750 & 0.025375 & 0.079225 & 0.011619 & 52.8125 \\ 31 & 0.008906 & 0.001931 & 0.278079 & 0.349969 & 0.203438 & 0.026156 & 0.121879 & 0.009643 & 35.6250 \\ 32 & 0.008203 & 0.020500 & 0.545637 & 0.059063 & 0.223125 & 0.026938 & 0.103598 & 0.012936 & 55.9375 \\ 33 & 0.007500 & 0.000650 & 0.558582 & 0.069094 & 0.242813 & 0.027719 & 0.085318 & 0.008325 & 32.5000\end{array}$

From the information of Table 5, there appear to be 14 initial design points that fail to satisfy the design interval for $\mathrm{CO}_{3}$ and, thus, must be discarded. Note that as each point is discarded one sub-interval for each of the inputs is no longer represented in the design. Also note that the orthogonality of the design is also impaired by the elimination of these design points. Thus, attaining the space-filling and orthogonality advantages inherent in the Latin hypercube method of [7] is hampered by the restrictions imposed on the design space through equation (1) and Table 1.

Although the special characteristics of this design problem lead to less than ideal results from the OLH approach, the space-filling feature of the design still has its advantages. In an attempt to select additional design points that help to fill-in the factor space, the OLH approach was repeated with the upper and lower limits for the values of $\mathrm{AlO}_{2}, \mathrm{C}_{2} \mathrm{O}_{4}$, $\mathrm{NO}_{2}, \mathrm{NO}_{3}, \mathrm{OH}, \mathrm{SO}_{4}$, and $\mathrm{TcO}_{4}$ moved in by $20 \%$ of their respective ranges. For example, instead of $\mathrm{AlO}_{2}$ covering the interval from 0.0075 to 0.0300 (as seen in Table 1 ), the factor was restricted to the interval from 0.0120 to 0.0255 . The results from the OLH approach are provided in Table 6 with the infeasible $\mathrm{CO}_{3}$ values shaded as in Table 5.

\section{Table 6: Secondary Orthogonal Latin Hypercube Design}

$\begin{array}{ccccccccccc}\text { Secondary } & \mathrm{AlO}_{2} & \mathrm{C}_{2} \mathrm{O}_{4} & \mathrm{CO}_{3} & \mathrm{NO}_{2} & \mathrm{NO}_{3} & \mathrm{OH} & \mathrm{SO}_{4} & \mathrm{TcO}_{4} & \text { Temperature } \\ \text { Design Pt } & (\mathrm{WF}) & (\mathrm{WF}) & (\mathrm{WF}) & (\mathrm{WF}) & (\mathrm{WF}) & (\mathrm{WF}) & (\mathrm{WF}) & (\mathrm{WF}) & \left({ }^{\circ} \mathrm{C}\right) \\ 1 & 0.019172 & 0.009487 & 0.280978 & 0.175425 & 0.325500 & 0.021000 & 0.152344 & 0.016094 & 59.0625\end{array}$




\begin{tabular}{|c|c|c|c|c|c|c|c|c|c|}
\hline 2 & 0.019594 & 0.010639 & 0.385112 & 0.181444 & 0.337313 & 0.021469 & 0.039010 & 0.005421 & 29.3750 \\
\hline 3 & 0.020016 & 0.008719 & 0.321803 & 0.211538 & 0.349125 & 0.021938 & 0.049978 & 0.016885 & 62.1875 \\
\hline 4 & 0.020438 & 0.011407 & 0.225975 & 0.205519 & 0.360938 & 0.022406 & 0.148688 & 0.004630 & 26.2500 \\
\hline 5 & 0.020859 & 0.007951 & 0.184598 & 0.151350 & 0.467250 & 0.022875 & 0.137720 & 0.007397 & 65.3125 \\
\hline 6 & 0.021281 & 0.012175 & 0.261852 & 0.157369 & 0.455438 & 0.023344 & 0.053634 & 0.014908 & 23.1250 \\
\hline 7 & 0.021703 & 0.007183 & 0.196856 & 0.235613 & 0.443625 & 0.023813 & 0.064602 & 0.006607 & 68.4375 \\
\hline 8 & 0.022125 & 0.012943 & 0.129481 & 0.229594 & 0.431813 & 0.024281 & 0.134064 & 0.015699 & 20.0000 \\
\hline 9 & 0.022547 & 0.006414 & 0.444486 & 0.127275 & 0.231000 & 0.032250 & 0.123097 & 0.012932 & 43.4375 \\
\hline 10 & 0.022969 & 0.013712 & 0.478592 & 0.133294 & 0.242813 & 0.031781 & 0.068258 & 0.008583 & 48.1250 \\
\hline 11 & 0.023391 & 0.005646 & 0.332391 & 0.259688 & 0.254625 & 0.031313 & 0.079225 & 0.013722 & 40.3125 \\
\hline 12 & 0.023813 & 0.014480 & 0.283525 & 0.253669 & 0.266438 & 0.030844 & 0.119441 & 0.007793 & 51.2500 \\
\hline 13 & 0.024234 & 0.004878 & 0.156530 & 0.103200 & 0.561750 & 0.030375 & 0.108473 & 0.010560 & 37.1875 \\
\hline 14 & 0.024656 & 0.015248 & 0.176407 & 0.109219 & 0.549938 & 0.029906 & 0.082881 & 0.011746 & 54.3750 \\
\hline 15 & 0.025078 & 0.004110 & 0.015869 & 0.283763 & 0.538125 & 0.029438 & 0.093849 & 0.009769 & 34.0625 \\
\hline 16 & 0.025500 & 0.016016 & 0.008106 & 0.277744 & 0.526313 & 0.028969 & 0.104817 & 0.012536 & 57.5000 \\
\hline 17 & 0.018750 & 0.010255 & 0.214535 & 0.199500 & 0.420000 & 0.028500 & 0.097505 & 0.010955 & 45.0000 \\
\hline 18 & 0.018328 & 0.011023 & 0.148092 & 0.223575 & 0.514500 & 0.036000 & 0.042666 & 0.005816 & 30.9375 \\
\hline 19 & 0.017906 & 0.009871 & 0.043958 & 0.217556 & 0.502688 & 0.035531 & 0.156000 & 0.016489 & 60.6250 \\
\hline 20 & 0.017484 & 0.011791 & 0.107267 & 0.187463 & 0.490875 & 0.035063 & 0.145032 & 0.005025 & 27.8125 \\
\hline 21 & 0.017063 & 0.009103 & 0.203095 & 0.193481 & 0.479063 & 0.034594 & 0.046322 & 0.017280 & 63.7500 \\
\hline 22 & 0.016641 & 0.012559 & 0.244473 & 0.247650 & 0.372750 & 0.034125 & 0.057290 & 0.014513 & 24.6875 \\
\hline 23 & 0.016219 & 0.008335 & 0.167218 & 0.241631 & 0.384563 & 0.033656 & 0.141376 & 0.007002 & 66.8750 \\
\hline 24 & 0.015797 & 0.013328 & 0.232214 & 0.163388 & 0.396375 & 0.033188 & 0.130408 & 0.015303 & 21.5625 \\
\hline 25 & 0.015375 & 0.007567 & 0.299589 & 0.169406 & 0.408188 & 0.032719 & 0.060946 & 0.006211 & 70.0000 \\
\hline 26 & 0.014953 & 0.014096 & -0.015416 & 0.271725 & 0.609000 & 0.024750 & 0.071913 & 0.008978 & 46.5625 \\
\hline 27 & 0.014531 & 0.006798 & -0.049522 & 0.265706 & 0.597188 & 0.025219 & 0.126753 & 0.013327 & 41.8750 \\
\hline 28 & 0.014109 & 0.014864 & 0.096679 & 0.139313 & 0.585375 & 0.025688 & 0.115785 & 0.008188 & 49.6875 \\
\hline
\end{tabular}




$\begin{array}{llllllllllll}29 & 0.013688 & 0.006030 & 0.145545 & 0.145331 & 0.573563 & 0.026156 & 0.075569 & 0.014118 & 38.7500 \\ 30 & 0.013266 & 0.015632 & 0.272540 & 0.295800 & 0.278250 & 0.026625 & 0.086537 & 0.011350 & 52.8125 \\ 31 & 0.012844 & 0.005262 & 0.252663 & 0.289781 & 0.290063 & 0.027094 & 0.112129 & 0.010164 & 35.6250 \\ 32 & 0.012422 & 0.016400 & 0.413201 & 0.115238 & 0.301875 & 0.027563 & 0.101161 & 0.012141 & 55.9375 \\ 33 & 0.012000 & 0.004494 & 0.420964 & 0.121256 & 0.313688 & 0.028031 & 0.090193 & 0.009374 & 32.5000\end{array}$

From the information of Table 6, there appear to be 11 secondary design points that fail to satisfy the design interval for $\mathrm{CO}_{3}$ and, thus, must be discarded. This leaves 22 points in Table 6 that do satisfy all of the constraints and conditions for this study.

These 22 points along with the 19 points of Table 5 provide some space-filling candidates for the test matrix. Figure 1 provides a set of scatter plots for these 41 points and Table 7 provides the pairwise correlations. 
Figure 1: Scatter Plots of the Design Points Generated in Two Phases Using the Orthogonal Latin Hypercube Approach

(Blue $\mathrm{x}$ - Phase 1 and Orange Open Square - Phase 2)

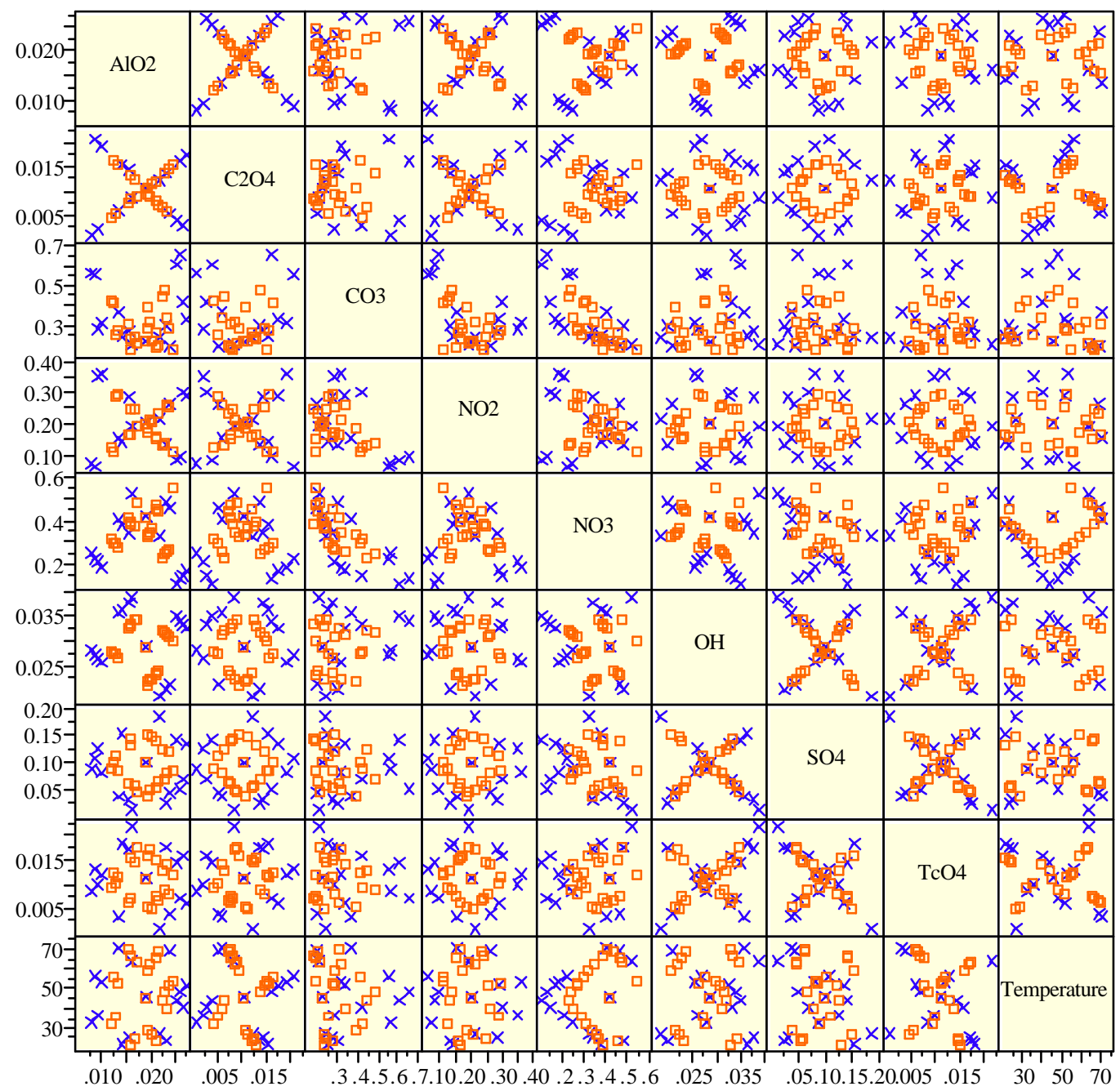


Table 7: Pairwise Correlations for the Initial and Secondary

Design Points Generated Using the Orthogonal Latin Hypercube Approach

$\begin{array}{cccccccccc} & \mathrm{AlO}_{2} & \mathrm{C}_{2} \mathrm{O}_{4} & \mathrm{CO}_{3} & \mathrm{NO}_{2} & \mathrm{NO}_{3} & \mathrm{OH} & \mathrm{SO}_{4} & \mathrm{TcO}_{4} & \text { Temperature } \\ \mathrm{AlO}_{2} & 1.0000 & 0.0095 & -0.0145 & -0.0236 & -0.0072 & -0.0490 & -0.0066 & -0.0972 & 0.0423 \\ \mathrm{C}_{2} \mathrm{O}_{4} & 0.0095 & 1.0000 & -0.0215 & -0.0557 & -0.0040 & 0.0005 & 0.0458 & 0.0953 & -0.0767 \\ \mathrm{CO}_{3} & -0.0145 & -0.0215 & 1.0000 & -0.4970 & -0.7408 & 0.1634 & -0.0596 & -0.0398 & -0.0981 \\ \mathrm{NO}_{2} & -0.0236 & -0.0557 & -0.4970 & 1.0000 & -0.1190 & -0.0637 & -0.0107 & -0.0669 & 0.0214 \\ \mathrm{NO}_{3} & -0.0072 & -0.0040 & -0.7408 & -0.1190 & 1.0000 & -0.1359 & -0.2850 & 0.1228 & 0.1619 \\ \mathrm{OH} & -0.0490 & 0.0005 & 0.1634 & -0.0637 & -0.1359 & 1.0000 & -0.1610 & 0.3343 & 0.0602 \\ \mathrm{SO}_{4} & -0.0066 & 0.0458 & -0.0596 & -0.0107 & -0.2850 & -0.1610 & 1.0000 & -0.2474 & -0.1669 \\ \mathrm{TcO}_{4} & -0.0972 & 0.0953 & -0.0398 & -0.0669 & 0.1228 & 0.3343 & -0.2474 & 1.0000 & -0.1898 \\ \mathrm{Temperature}^{2} & 0.0423 & -0.0767 & -0.0981 & 0.0214 & 0.1619 & 0.0602 & -0.1669 & -0.1898 & 1.0000\end{array}$

\subsection{A Sequential Approach for Model Building}

A large number of candidate design points has been selected for consideration in the development of this test matrix. These points will be reviewed and supplemented in this section. The use of these points in the development and validation of models for the responses generated by the OLI/ESP computer algorithms is to be a sequential process, which is also outlined in this section.

\subsubsection{The First Set of Test Runs}

The initial set of test runs is to be used to develop a linear model in the mixture components and to explore the need for temperature (the process variable) terms in this model. Thus, only a subset of the terms in the model given by equation (2) is of interest initially. This reduced model (maintaining the same numbering scheme for the $\beta$ 's) is given by equation (3).

$$
\begin{aligned}
\text { Response }_{\mathrm{ESP} / \mathrm{OLI}} & =\beta_{1} \mathrm{AlO}_{2}+\beta_{2} \mathrm{C}_{2} \mathrm{O}_{4}+\beta_{4} \mathrm{CO}_{3}+\beta_{7} \mathrm{NO}_{2}+\beta_{11} \mathrm{NO}_{3}+\beta_{16} \mathrm{OH} \\
& +\beta_{22} \mathrm{SO}_{4}+\beta_{29} \mathrm{TcO}_{4}+\beta_{37} \mathrm{AlO}_{2} \cdot \mathrm{Temp}+\beta_{38} \mathrm{C}_{2} \mathrm{O}_{4} \cdot \text { Temp } \\
& +\beta_{40} \mathrm{CO}_{3} \cdot \mathrm{Temp}+\beta_{43} \mathrm{NO}_{2} \cdot \mathrm{Temp}+\beta_{47} \mathrm{NO}_{3} \cdot \text { Temp } \\
& +\beta_{52} \mathrm{OH} \cdot \mathrm{Temp}+\beta_{58} \mathrm{SO}_{4} \cdot \mathrm{Temp}+\beta_{65} \mathrm{TcO}_{4} \cdot \text { Temp }
\end{aligned}
$$


There are 16 terms (unknown coefficients) in equation (3), which require at least 16 computer runs to generate the data so that these coefficients can be estimated.

For a strictly linear mixture model, the optimal design points are a subset of the extreme vertices of the mixture factor space. A review (in the form of a table of minimums and maximums) of all of the candidate design points developed above is provided in Table 8 . 
Table 8: Minimums and Maximums of Candidate Design Points Along with the Initial Factor Intervals Defining the Design Space (Chemical Species in Weight Fractions, WFs)

\begin{tabular}{|ccccccccccccc|}
\hline & & $\mathrm{AlO}_{2}$ & $\mathrm{C}_{2} \mathrm{O}_{4}$ & $\mathrm{CO}_{3}$ & $\mathrm{NO}_{2}$ & $\mathrm{NO}_{3}$ & $\mathrm{OH}$ & $\mathrm{SO}_{4}$ & $\mathrm{TcO}_{4}$ & Temperature \\
& & $(\mathrm{WF})$ & $(\mathrm{WF})$ & $(\mathrm{WF})$ & $(\mathrm{WF})$ & $(\mathrm{WF})$ & $(\mathrm{WF})$ & $(\mathrm{WF})$ & $(\mathrm{WF})$ & $\left({ }^{\circ} \mathrm{C}\right)$ \\
\hline Candidate & $\mathrm{Min}$ & 0.00750 & 0.00001 & 0.16722 & 0.03900 & 0.10500 & 0.01600 & 0.00001 & 0.00042 & 20 \\
Points & $\mathrm{Max}$ & 0.03000 & 0.02050 & 0.78657 & 0.36000 & 0.54994 & 0.04100 & 0.19500 & 0.02150 & 70 \\
\hline Factor & $\operatorname{Min}$ & 0.00750 & 0.00001 & 0.16500 & 0.03900 & 0.10500 & 0.01600 & 0.00001 & 0.00042 & 20 \\
Interval & $\operatorname{Max}$ & 0.03000 & 0.02050 & 0.80500 & 0.36000 & 0.73500 & 0.04100 & 0.19500 & 0.02150 & 70 \\
\hline
\end{tabular}

From Table 8, it is evident that the candidate points considered so far do not cover the maximums for $\mathrm{CO}_{3}$ and $\mathrm{NO}_{3}$. To remedy this problem, the set of extreme vertices of the factor space defined by the mixture components of Table 1 was generated and a set of 8 design points was optimally selected (using the D-Optimality Routine of JMP Version 3.2.6) to support the estimation of the mixture terms of equation (3). As this process was conducted, the JMP routines were utilized in such a manner as to ensure that the desired maximums were included in the final 8 design points. These optimal mixtures are to be run at both the low extreme and high extreme of temperature (as shown in Table 9) to generate the necessary data for fitting the model given by equation (3).

Table 9: First Set of Computer Runs in Weight Fraction (WFs) Units

$\begin{array}{ccccccccccc}\text { Run } & \mathrm{AlO}_{2} & \mathrm{C}_{2} \mathrm{O}_{4} & \mathrm{CO}_{3} & \mathrm{NO}_{2} & \mathrm{NO}_{3} & \mathrm{OH} & \mathrm{SO}_{4} & \mathrm{TcO}_{4} & \text { Temperature } \\ \text { ID } & (\mathrm{WF}) & (\mathrm{WF}) & (\mathrm{WF}) & (\mathrm{WF}) & (\mathrm{WF}) & (\mathrm{WF}) & (\mathrm{WF}) & (\mathrm{WF}) & \left({ }^{\circ} \mathrm{C}\right) \\ \mathrm{RTS} 001 & 0.007500 & 0.000010 & 0.805000 & 0.039000 & 0.107060 & 0.041000 & 0.000010 & 0.000420 & 20 & 20 \\ \text { RTS002 } & 0.007500 & 0.020500 & 0.595500 & 0.039000 & 0.105000 & 0.016000 & 0.195000 & 0.021500 & 20 & 20 \\ \text { RTS003 } & 0.030000 & 0.020500 & 0.421990 & 0.360000 & 0.105000 & 0.041000 & 0.000010 & 0.021500 & 20 \\ \text { RTS004 } & 0.030000 & 0.000010 & 0.293570 & 0.360000 & 0.105000 & 0.016000 & 0.195000 & 0.000420 & 20 \\ \text { RTS005 } & 0.007500 & 0.020500 & 0.165000 & 0.360000 & 0.430570 & 0.016000 & 0.000010 & 0.000420 & 20 \\ \text { RTS006 } & 0.007500 & 0.000010 & 0.165000 & 0.360000 & 0.209990 & 0.041000 & 0.195000 & 0.021500 & 20\end{array}$




$\begin{array}{lccccccccccc}\text { RTS007 } & 0.023480 & 0.000010 & 0.165000 & 0.039000 & 0.735000 & 0.016000 & 0.000010 & 0.021500 & 20 \\ \text { RTS008 } & 0.030000 & 0.020500 & 0.165000 & 0.039000 & 0.509080 & 0.041000 & 0.195000 & 0.000420 & 20 \\ \text { RTS009 } & 0.007500 & 0.000010 & 0.805000 & 0.039000 & 0.107060 & 0.041000 & 0.000010 & 0.000420 & 70 \\ \text { RTS010 } & 0.007500 & 0.020500 & 0.595500 & 0.039000 & 0.105000 & 0.016000 & 0.195000 & 0.021500 & 70 \\ \text { RTS011 } & 0.030000 & 0.020500 & 0.421990 & 0.360000 & 0.105000 & 0.041000 & 0.000010 & 0.021500 & 70 \\ \text { RTS012 } & 0.030000 & 0.000010 & 0.293570 & 0.360000 & 0.105000 & 0.016000 & 0.195000 & 0.000420 & 70 \\ \text { RTS013 } & 0.007500 & 0.020500 & 0.165000 & 0.360000 & 0.430570 & 0.016000 & 0.000010 & 0.000420 & 70 \\ \text { RTS014 } & 0.007500 & 0.000010 & 0.165000 & 0.360000 & 0.209990 & 0.041000 & 0.195000 & 0.021500 & 70 \\ \text { RTS015 } & 0.023480 & 0.000010 & 0.165000 & 0.039000 & 0.735000 & 0.016000 & 0.000010 & 0.021500 & 70 \\ \text { RTS016 } & 0.030000 & 0.020500 & 0.165000 & 0.039000 & 0.509080 & 0.041000 & 0.195000 & 0.000420 & 70\end{array}$

The efficiencies and other details of this selection of 8 extreme vertices of the factor space are provided in Figure 2 (see [2] for a discussion of the details of this format). 
Figure 2: Results from the D-Optimality Routine of JMP Version 3.2.6

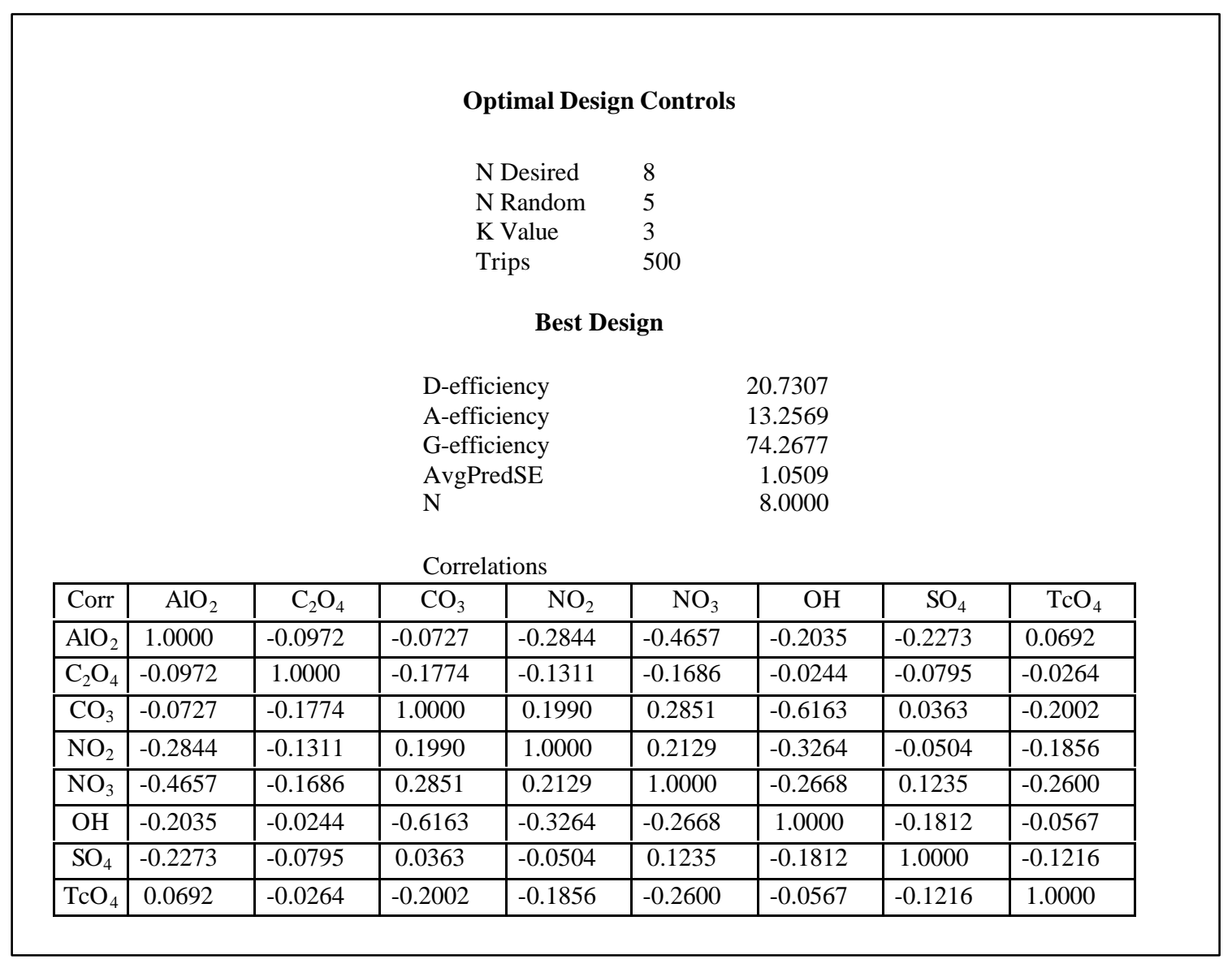

\subsubsection{The Second Set of Test Runs}

Upon completion of the first set of 16 computer runs, activities are to be conducted on two parallel paths: the data from the first set of computer runs are to be investigated as a second set of computer runs is to be initiated. The second set of computer runs are to be the 41 design points generated using the OLH approach of Section 2.4. These runs are identified in Table 10. 


\section{Table 10: Second Set of Computer Runs in Weight Fractions (WFs)}

$\begin{array}{cccccccccc}\text { Run } & \mathrm{AlO}_{2} & \mathrm{C}_{2} \mathrm{O}_{4} & \mathrm{CO}_{3} & \mathrm{NO}_{2} & \mathrm{NO}_{3} & \mathrm{OH} & \mathrm{SO}_{4} & \mathrm{TcO}_{4} & \text { Temperature } \\ \text { ID } & (\mathrm{WF}) & (\mathrm{WF}) & (\mathrm{WF}) & (\mathrm{WF}) & (\mathrm{WF}) & (\mathrm{WF}) & (\mathrm{WF}) & (\mathrm{WF}) & \left({ }^{\circ} \mathrm{C}\right)\end{array}$

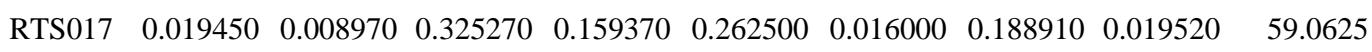

$\begin{array}{llllllllll}\text { RTS018 } & 0.020160 & 0.010900 & 0.498830 & 0.169410 & 0.282190 & 0.016780 & 0.000010 & 0.001740 & 29.3750\end{array}$

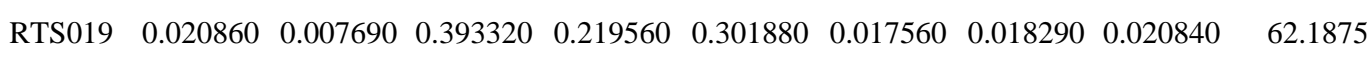

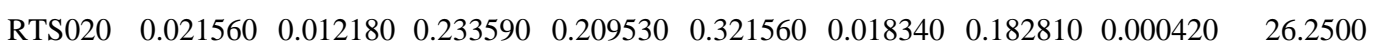

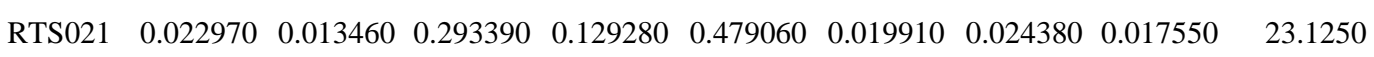

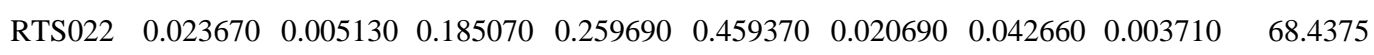

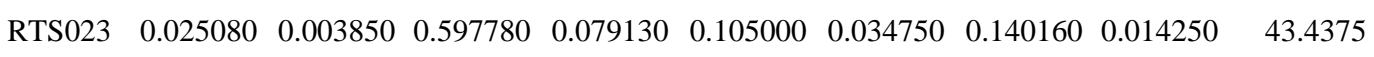

$\begin{array}{llllllllll}\text { RTS024 } & 0.025780 & 0.016020 & 0.654620 & 0.089160 & 0.124690 & 0.033970 & 0.048760 & 0.007010 & 48.1250\end{array}$

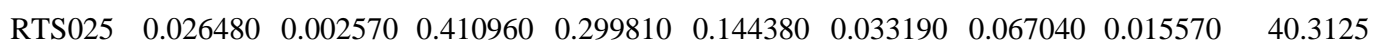

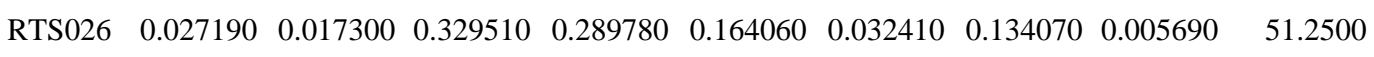

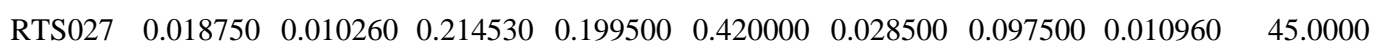

$\begin{array}{llllllllll}\text { RTS028 } & 0.015940 & 0.008330 & 0.195470 & 0.189470 & 0.518440 & 0.038660 & 0.012200 & 0.021500 & 63.7500\end{array}$

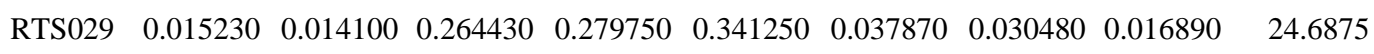

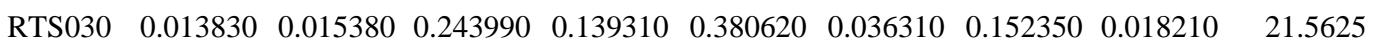

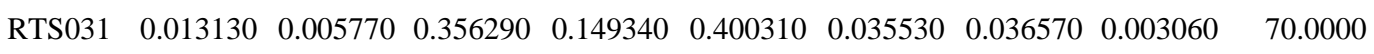

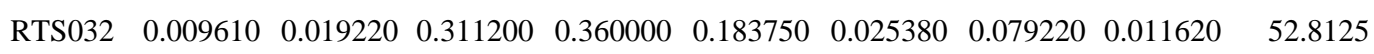

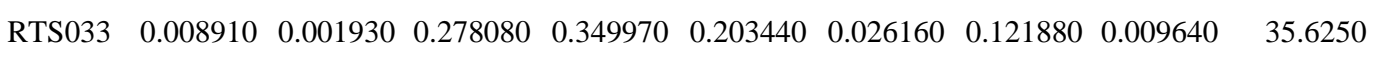

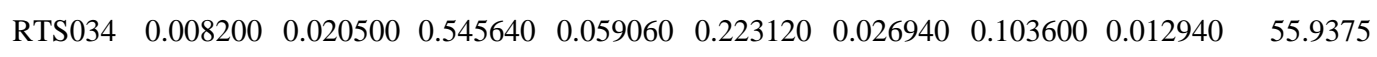

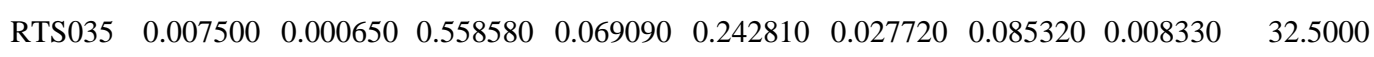

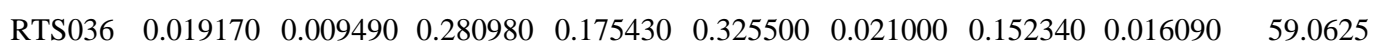

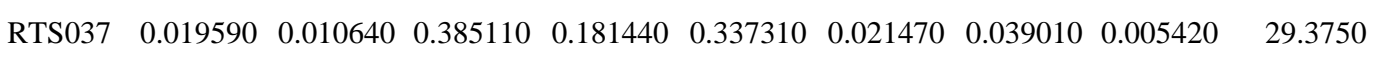

$\begin{array}{llllllllll}\text { RTS038 } & 0.020020 & 0.008720 & 0.321800 & 0.211540 & 0.349130 & 0.021940 & 0.049980 & 0.016880 & 62.1875\end{array}$

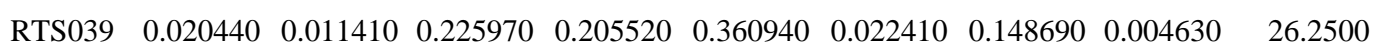

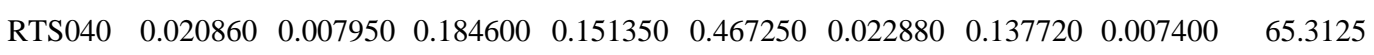




\begin{tabular}{|c|c|c|c|c|c|c|c|c|c|}
\hline RTS041 & 0.021280 & 0.012180 & 0.261850 & 0.157370 & 0.455440 & 0.023340 & 0.053630 & 0.014910 & 23.1250 \\
\hline RTS042 & 0.021700 & 0.007180 & 0.196860 & 0.235610 & 0.443620 & 0.023810 & 0.064600 & 0.006610 & 68.4375 \\
\hline TS042 & 0.022550 & 0.006410 & 0.444490 & 0.127280 & 0.231000 & 0.032250 & 0.123100 & 0.012930 & 43.4375 \\
\hline RTS044 & 0.022970 & 0.013710 & 0.478590 & 0.133290 & 0.242810 & 0.031780 & 0.068260 & 0.008580 & 48.1250 \\
\hline RTS045 & 0.023390 & 0.005650 & 0.332390 & 0.259690 & 0.254620 & 0.031310 & 0.079230 & 0.013720 & 40.3125 \\
\hline RTS046 & 0.023810 & 0.014480 & 0.283520 & 0.253670 & 0.266440 & 0.030840 & 0.119440 & 0.007790 & 51.2500 \\
\hline RTS047 & 0.024660 & 0.015250 & 0.176410 & 0.109220 & 0.549940 & 0.029910 & 0.082880 & 0.011750 & 54.3750 \\
\hline RTS048 & 0.018750 & 0.010260 & 0.214540 & 0.199500 & 0.420000 & 0.028500 & 0.097500 & 0.010950 & 45.0000 \\
\hline RTS049 & .017060 & 0.009100 & 0.203100 & 0.193480 & 0.479060 & 0.034590 & 0.046320 & 0.017280 & 63.7500 \\
\hline RTS050 & 0.016640 & 0.012560 & 0.244470 & 0.247650 & 0.372750 & 0.034130 & 0.057290 & 0.014510 & 24.6875 \\
\hline RTS051 & 0.016220 & 0.008330 & 0.167220 & 0.241630 & 0.384560 & 0.033660 & 0.141380 & 0.007000 & 66.8750 \\
\hline RTS052 & 0.015800 & 0.013330 & 0.232210 & 0.163390 & 0.396370 & 0.033190 & 0.130410 & 0.015300 & 21.5 \\
\hline RTS053 & 0.015380 & 0.007570 & 0.299590 & 0.169410 & 0.408190 & 0.032720 & 0.060950 & 0.006210 & 70.0000 \\
\hline RTS054 & 0.013270 & 0.015630 & 0.272540 & 0.295800 & 0.278250 & 0.026620 & 0.086540 & 0.011350 & 52.8125 \\
\hline RTS055 & 0.012840 & 0.005260 & 0.252660 & 0.289780 & 0.290060 & 0.027090 & 0.112130 & 0.010160 & 35.62 \\
\hline RTS056 & 0.012420 & 0.016400 & 0.413200 & 0.115240 & 0.301880 & 0.027560 & 0.101160 & 0.012140 & 55.9 \\
\hline TS057 & 0.012000 & 0.004490 & 0.420960 & 0.121260 & 0.313690 & 0.028030 & 0.090190 & 0.009370 & 0 \\
\hline
\end{tabular}

If the modeling efforts associated with the first set of responses (those generated from the input data identified in Table 9) look promising, the results from running the test cases of Table 10 will be used to evaluate the performance of these models.

\subsubsection{The Third and Final Set of Test Runs}

If models consisting only of linear mixture terms (such as that given by the linear mixture terms of equation (3)) appear to be inadequate to explain all of the variation in the responses of interest, additional computer runs are to be conducted to support the fitting of more complex models (such as that given by equation (2)). The additional computer runs will be the 36 mixture design points that were selected in Section 2.3.

If temperature appears to be a significance factor (based upon the data generated from the computer runs of Section 2.5.1), then the 36 mixture design points should be run at 20 and at $70^{\circ} \mathrm{C}$. If temperature does not appear to be significant, then the 36 mixture points 
can be run at a single temperature. Table 11 provides the complete set ( 72 design points) of computer runs that cover these situations.

\section{Table 11: Third Set of Computer Runs in Weight Fraction (WFs)}

\begin{tabular}{|c|c|c|c|c|c|c|c|c|c|}
\hline Run & $\mathrm{AlO}_{2}$ & $\mathrm{C}_{2} \mathrm{O}_{4}$ & $\mathrm{CO}_{3}$ & $\mathrm{NO}_{2}$ & $\mathrm{NO}_{3}$ & $\mathrm{OH}$ & $\mathrm{SO}_{4}$ & $\mathrm{TcO}_{4}$ & Temperature \\
\hline ID & (WF) & (WF) & (WF) & (WF) & (WF) & (WF) & (WF) & (WF) & $\left({ }^{\circ} \mathrm{C}\right)$ \\
\hline RTS058 & 0.030000 & 0.020500 & 0.386840 & 0.039000 & 0.482230 & 0.041000 & 0.000010 & 0.000420 & 20 \\
\hline RTS059 & 0.017230 & 0.015280 & 0.615980 & 0.074080 & 0.233980 & 0.018980 & 0.002990 & 0.021490 & 20 \\
\hline RTS060 & 0.029990 & 0.020020 & 0.566960 & 0.121160 & 0.185960 & 0.036460 & 0.017970 & 0.021490 & 20 \\
\hline RTS061 & 0.029990 & 0.020490 & 0.453660 & 0.200060 & 0.200660 & 0.040990 & 0.032670 & 0.021490 & 20 \\
\hline RTS062 & 0.030000 & 0.000010 & 0.490320 & 0.233130 & 0.157840 & 0.016000 & 0.072280 & 0.000420 & 20 \\
\hline RTS063 & 0.029990 & 0.020500 & 0.746710 & 0.073670 & 0.107570 & 0.016000 & 0.005140 & 0.000420 & 20 \\
\hline RTS064 & 0.007500 & 0.020500 & 0.303180 & 0.360000 & 0.112690 & 0.041000 & 0.154710 & 0.000420 & 20 \\
\hline RTS065 & 0.029990 & 0.020490 & 0.390880 & 0.136880 & 0.202880 & 0.040990 & 0.156390 & 0.021490 & 20 \\
\hline RTS066 & 0.007500 & 0.020500 & 0.631250 & 0.174450 & 0.112770 & 0.024010 & 0.029100 & 0.000420 & 20 \\
\hline RTS067 & 0.029990 & 0.020490 & 0.358780 & 0.233180 & 0.170780 & 0.040990 & 0.124290 & 0.021490 & 20 \\
\hline RTS068 & 0.007500 & 0.000010 & 0.571190 & 0.104880 & 0.105000 & 0.016000 & 0.195000 & 0.000420 & 20 \\
\hline RTS069 & 0.007500 & 0.020500 & 0.786570 & 0.039000 & 0.105000 & 0.041000 & 0.000010 & 0.000420 & 20 \\
\hline RTS070 & 0.007500 & 0.020500 & 0.472620 & 0.181310 & 0.253820 & 0.041000 & 0.001740 & 0.021500 & 20 \\
\hline RTS071 & 0.029990 & 0.020490 & 0.286480 & 0.128580 & 0.414480 & 0.040990 & 0.057490 & 0.021490 & 20 \\
\hline RTS072 & 0.019980 & 0.000020 & 0.735480 & 0.065580 & 0.162480 & 0.016010 & 0.000020 & 0.000430 & 20 \\
\hline RTS073 & 0.029990 & 0.020490 & 0.277710 & 0.215910 & 0.280710 & 0.040990 & 0.112720 & 0.021490 & 20 \\
\hline RTS074 & 0.030000 & 0.020500 & 0.510300 & 0.109590 & 0.300390 & 0.016000 & 0.012790 & 0.000420 & 20 \\
\hline RTS075 & 0.030000 & 0.000010 & 0.541320 & 0.144900 & 0.208070 & 0.041000 & 0.013200 & 0.021500 & 20 \\
\hline RTS076 & 0.029990 & 0.020490 & 0.295330 & 0.169530 & 0.297330 & 0.040990 & 0.145910 & 0.000420 & 20 \\
\hline RTS077 & 0.029990 & 0.020490 & 0.525430 & 0.175730 & 0.145430 & 0.040990 & 0.061510 & 0.000420 & 20 \\
\hline RTS078 & 0.029990 & 0.009960 & 0.622950 & 0.145250 & 0.114950 & 0.025950 & 0.029460 & 0.021490 & 20 \\
\hline RTS079 & 0.029910 & 0.020490 & 0.569160 & 0.155460 & 0.125160 & 0.038660 & 0.039670 & 0.021490 & 20 \\
\hline
\end{tabular}




\begin{tabular}{|c|c|c|c|c|c|c|c|c|c|}
\hline RTS080 & 0.007500 & 0.000010 & 0.391020 & 0.039000 & 0.425420 & 0.016000 & 0.120630 & 0.000420 & 20 \\
\hline RTS081 & 0.029990 & 0.020490 & 0.332160 & 0.206560 & 0.270160 & 0.040990 & 0.078170 & 0.021490 & 20 \\
\hline RTS082 & 0.029990 & 0.020490 & 0.340180 & 0.182480 & 0.278180 & 0.040990 & 0.086190 & 0.021490 & 20 \\
\hline RTS083 & 0.030000 & 0.000010 & 0.768730 & 0.039000 & 0.120830 & 0.041000 & 0.000010 & 0.000420 & 20 \\
\hline RTS084 & 0.029990 & 0.014950 & 0.689890 & 0.083990 & 0.117890 & 0.028890 & 0.012900 & 0.021490 & 20 \\
\hline RTS085 & 0.029990 & 0.020490 & 0.567360 & 0.121560 & 0.186360 & 0.034360 & 0.018370 & 0.021490 & 20 \\
\hline RTS086 & 0.030000 & 0.020480 & 0.347530 & 0.136660 & 0.390460 & 0.019910 & 0.033470 & 0.021500 & 20 \\
\hline RTS087 & 0.007500 & 0.000010 & 0.669020 & 0.092660 & 0.193300 & 0.016000 & 0.000010 & 0.021500 & 20 \\
\hline RTS088 & 0.024820 & 0.020490 & 0.689820 & 0.083920 & 0.117820 & 0.028820 & 0.012830 & 0.021490 & 20 \\
\hline RTS089 & 0.029990 & 0.019850 & 0.682490 & 0.108690 & 0.110490 & 0.021490 & 0.005500 & 0.021490 & 20 \\
\hline RTS090 & 0.007500 & 0.000010 & 0.318720 & 0.160980 & 0.445680 & 0.040990 & 0.025690 & 0.000420 & 20 \\
\hline RTS091 & 0.011160 & 0.015960 & 0.744660 & 0.074760 & 0.108660 & 0.019660 & 0.003670 & 0.021490 & 20 \\
\hline RTS092 & 0.030000 & 0.020500 & 0.410960 & 0.212960 & 0.251260 & 0.041000 & 0.032910 & 0.000420 & 20 \\
\hline RTS093 & 0.029990 & 0.020490 & 0.485760 & 0.103760 & 0.232760 & 0.040990 & 0.064770 & 0.021490 & 20 \\
\hline RTS094 & 0.030000 & 0.020500 & 0.386840 & 0.039000 & 0.482230 & 0.041000 & 0.000010 & 0.000420 & 70 \\
\hline RTS095 & 0.017230 & 0.015280 & 0.615980 & 0.074080 & 0.233980 & 0.018980 & 0.002990 & 0.021490 & 70 \\
\hline RTS096 & 0.029990 & 0.020020 & 0.566960 & 0.121160 & 0.185960 & 0.036460 & 0.017970 & 0.021490 & 70 \\
\hline RTS097 & 0.029990 & 0.020490 & 0.453660 & 0.200060 & 0.200660 & 0.040990 & 0.032670 & 0.021490 & 70 \\
\hline RTS098 & 0.030000 & 0.000010 & 0.490320 & 0.233130 & 0.157840 & 0.016000 & 0.072280 & 0.000420 & 70 \\
\hline RTS099 & 0.029990 & 0.020500 & 0.746710 & 0.073670 & 0.107570 & 0.016000 & 0.005140 & 0.000420 & 70 \\
\hline RTS100 & 0.007500 & 0.020500 & 0.303180 & 0.360000 & 0.112690 & 0.041000 & 0.154710 & 0.000420 & 70 \\
\hline RTS101 & 0.029990 & 0.020490 & 0.390880 & 0.136880 & 0.202880 & 0.040990 & 0.156390 & 0.021490 & 70 \\
\hline RTS102 & 0.007500 & 0.020500 & 0.631250 & 0.174450 & 0.112770 & 0.024010 & 0.029100 & 0.000420 & 70 \\
\hline
\end{tabular}


Table 11: Third Set of Computer Runs in Weight Fraction (WFs)

\begin{tabular}{|c|c|c|c|c|c|c|c|c|c|}
\hline \multicolumn{10}{|c|}{ (Continued) } \\
\hline Run & $\mathrm{AlO}_{2}$ & $\mathrm{C}_{2} \mathrm{O}_{4}$ & $\mathrm{CO}_{3}$ & $\mathrm{NO}_{2}$ & $\mathrm{NO}_{3}$ & $\mathrm{OH}$ & $\mathrm{SO}_{4}$ & $\mathrm{TcO}_{4}$ & Temperature \\
\hline ID & (WF) & (WF) & (WF) & $(\mathrm{WF})$ & (WF) & (WF) & (WF) & $(\mathrm{WF})$ & $\left({ }^{\circ} \mathrm{C}\right)$ \\
\hline RTS103 & 0.029990 & 0.020490 & 0.358780 & 0.233180 & 0.170780 & 0.040990 & 0.124290 & 0.021490 & 70 \\
\hline RTS104 & 0.007500 & 0.000010 & 0.571190 & 0.104880 & 0.105000 & 0.016000 & 0.195000 & 0.000420 & 70 \\
\hline RTS105 & 0.007500 & 0.020500 & 0.786570 & 0.039000 & 0.105000 & 0.041000 & 0.000010 & 0.000420 & 70 \\
\hline RTS106 & 0.007500 & 0.020500 & 0.472620 & 0.181310 & 0.253820 & 0.041000 & 0.001740 & 0.021500 & 70 \\
\hline RTS107 & 0.029990 & 0.020490 & 0.286480 & 0.128580 & 0.414480 & 0.040990 & 0.057490 & 0.021490 & 70 \\
\hline RTS108 & 0.019980 & 0.000020 & 0.735480 & 0.065580 & 0.162480 & 0.016010 & 0.000020 & 0.000430 & 70 \\
\hline RTS109 & 0.029990 & 0.020490 & 0.277710 & 0.215910 & 0.280710 & 0.040990 & 0.112720 & 0.021490 & 70 \\
\hline RTS110 & 0.030000 & 0.020500 & 0.510300 & 0.109590 & 0.300390 & 0.016000 & 0.012790 & 0.000420 & 70 \\
\hline RTS111 & 0.030000 & 0.000010 & 0.541320 & 0.144900 & 0.208070 & 0.041000 & 0.013200 & 0.021500 & 70 \\
\hline RTS112 & 0.029990 & 0.020490 & 0.295330 & 0.169530 & 0.297330 & 0.040990 & 0.145910 & 0.000420 & 70 \\
\hline RTS113 & 0.029990 & 0.020490 & 0.525430 & 0.175730 & 0.145430 & 0.040990 & 0.061510 & 0.000420 & 70 \\
\hline RTS114 & 0.029990 & 0.009960 & 0.622950 & 0.145250 & 0.114950 & 0.025950 & 0.029460 & 0.021490 & 70 \\
\hline RTS115 & 0.029910 & 0.020490 & 0.569160 & 0.155460 & 0.125160 & 0.038660 & 0.039670 & 0.021490 & 70 \\
\hline RTS116 & 0.007500 & 0.000010 & 0.391020 & 0.039000 & 0.425420 & 0.016000 & 0.120630 & 0.000420 & 70 \\
\hline RTS117 & 0.029990 & 0.020490 & 0.332160 & 0.206560 & 0.270160 & 0.040990 & 0.078170 & 0.021490 & 70 \\
\hline RTS118 & 0.029990 & 0.020490 & 0.340180 & 0.182480 & 0.278180 & 0.040990 & 0.086190 & 0.021490 & 70 \\
\hline RTS119 & 0.030000 & 0.000010 & 0.768730 & 0.039000 & 0.120830 & 0.041000 & 0.000010 & 0.000420 & 70 \\
\hline RTS120 & 0.029990 & 0.014950 & 0.689890 & 0.083990 & 0.117890 & 0.028890 & 0.012900 & 0.021490 & 70 \\
\hline RTS121 & 0.029990 & 0.020490 & 0.567360 & 0.121560 & 0.186360 & 0.034360 & 0.018370 & 0.021490 & 70 \\
\hline RTS122 & 0.030000 & 0.020480 & 0.347530 & 0.136660 & 0.390460 & 0.019910 & 0.033470 & 0.021500 & 70 \\
\hline RTS123 & 0.007500 & 0.000010 & 0.669020 & 0.092660 & 0.193300 & 0.016000 & 0.000010 & 0.021500 & 70 \\
\hline RTS124 & 0.024820 & 0.020490 & 0.689820 & 0.083920 & 0.117820 & 0.028820 & 0.012830 & 0.021490 & 70 \\
\hline RTS125 & 0.029990 & 0.019850 & 0.682490 & 0.108690 & 0.110490 & 0.021490 & 0.005500 & 0.021490 & 70 \\
\hline
\end{tabular}


$\begin{array}{lllllllllll}\text { RTS126 } & 0.007500 & 0.000010 & 0.318720 & 0.160980 & 0.445680 & 0.040990 & 0.025690 & 0.000420 & 70 \\ \text { RTS127 } & 0.011160 & 0.015960 & 0.744660 & 0.074760 & 0.108660 & 0.019660 & 0.003670 & 0.021490 & 70 \\ \text { RTS128 } & 0.030000 & 0.020500 & 0.410960 & 0.212960 & 0.251260 & 0.041000 & 0.032910 & 0.000420 & 70 \\ \text { RTS129 } & 0.029990 & 0.020490 & 0.485760 & 0.103760 & 0.232760 & 0.040990 & 0.064770 & 0.021490 & 70\end{array}$

The 72 design points of Table 11 along with the first set of 16 test runs (in Table 9) provide all of the data necessary to conduct a model fit for equation (2) for each response of interest. The data from Table 10 provide the information necessary to assess the performance of the models. 


\subsubsection{The Complete Test Matrix}

Tables 9, 10, and 11 define a 3-phased test matrix for this Tc solubility study. Figure 3 provides a scatter of all of the design points outlined in this sequential testing approach. Table 12 provides the pairwise correlations among these data. Exhibit 1 in the Appendix provides additional details of the coverage of the factor space defined by Table 1 for the full set of 129 potential design points.

Table 12: Pairwise Correlations of Design Points

$\begin{array}{cccccccccc} & \mathrm{AlO}_{2} & \mathrm{C}_{2} \mathrm{O}_{4} & \mathrm{CO}_{3} & \mathrm{NO}_{2} & \mathrm{NO}_{3} & \mathrm{OH} & \mathrm{SO}_{4} & \mathrm{TcO}_{4} & \text { Temperature } \\ \mathrm{AlO}_{2} & 1.0000 & 0.3189 & 0.0056 & -0.0477 & -0.0388 & 0.2355 & -0.1126 & 0.2065 & 0.0007 \\ \mathrm{C}_{2} \mathrm{O}_{4} & 0.3189 & 1.0000 & 0.0264 & 0.0052 & -0.1130 & 0.3085 & -0.0905 & 0.2219 & -0.0181 \\ \mathrm{CO}_{3} & 0.0056 & 0.0264 & 1.0000 & -0.5612 & -0.7300 & -0.1008 & -0.4768 & 0.0008 & -0.0270 \\ \mathrm{NO}_{2} & -0.0477 & 0.0052 & -0.5612 & 1.0000 & -0.0437 & 0.1063 & 0.2703 & -0.0116 & 0.0107 \\ \mathrm{NO}_{3} & -0.0388 & -0.1130 & -0.7300 & -0.0437 & 1.0000 & -0.0568 & 0.0188 & -0.0458 & 0.0338 \\ \mathrm{OH} & 0.2355 & 0.3085 & -0.1008 & 0.1063 & -0.0568 & 1.0000 & 0.0134 & 0.1446 & -0.0021 \\ \mathrm{SO}_{4} & -0.1126 & -0.0905 & -0.4768 & 0.2703 & 0.0188 & 0.0134 & 1.0000 & -0.1108 & -0.0084 \\ \mathrm{TcO}_{4} & 0.2065 & 0.2219 & 0.0008 & -0.0116 & -0.0458 & 0.1446 & -0.1108 & 1.0000 & -0.0060 \\ \mathrm{Temperature}^{2} & 0.0007 & -0.0181 & -0.0270 & 0.0107 & 0.0338 & -0.0021 & -0.0084 & -0.0060 & 1.0000\end{array}$


Figure 3: Scatter Plots of Final Design Points:

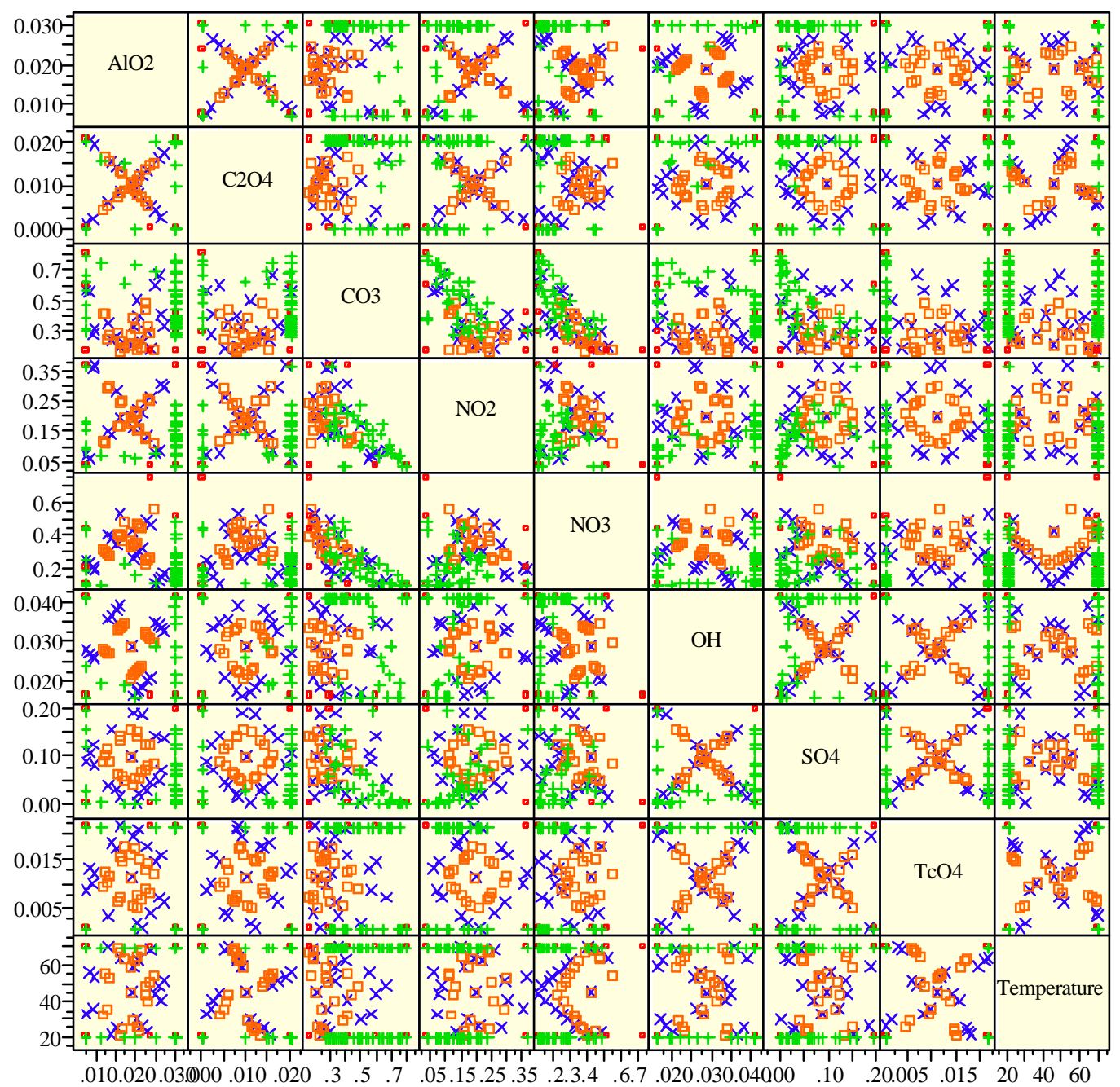

Legend

Type

- JMP Linear

* JMP MRSM

x OLH 1

a $\mathrm{OLH} 2$

\subsection{Concluding Comments}

A statistical design is provided for a sequential model development effort in support of the Tc eluate solubility study in this memorandum. Up to 3 stages of computer runs, a total of 129 design points in all, were identified. Successful completion of these 
experiments should provide the data necessary to fit and evaluate models for the responses (of interest) generated from the OLI/ESP software.

\subsection{Reference}

[1] SAS Institute, Inc., JMP® Statistics and Graphics Guide, Version 3, SAS Institute, Inc., Cary, NC, 1994.

[2] SAS Institute, Inc., JMP® Statistics and Graphics Guide, Version 4, SAS Institute, Inc., Cary, NC, 2000.

[3] Cornell, J. A. Experiments with Mixtures: Designs, Models, and the Analysis of Mixture Data, Second Edition, John Wiley \& Sons, Inc., New York, 1990.

[4] Iman, R. L. and J. C. Helton, "An Investigation of Uncertainty and Sensitivity Analysis Techniques for Computer Models," Risk Analysis, 8, 71-90, 1988.

[5] Sacks, J., S. B. Schiller, and W. J. Welch, "Designs for Computer Experiments," Technometrics, 31, 41-47,1989.

[6] Sacks, J., W. J. Welch, T. J. Mitchell, and H. P. Wynn, "Design and Analysis of Computer Experiments," Statistical Science, 4, 409-435, 1989. Experiments," Journal of the American Statistical Association, 93, 1430-1439, 1998. 


\section{Appendix.}

Exhibit A1: Histograms of Input Values for the Final Test Matrix

AlO2

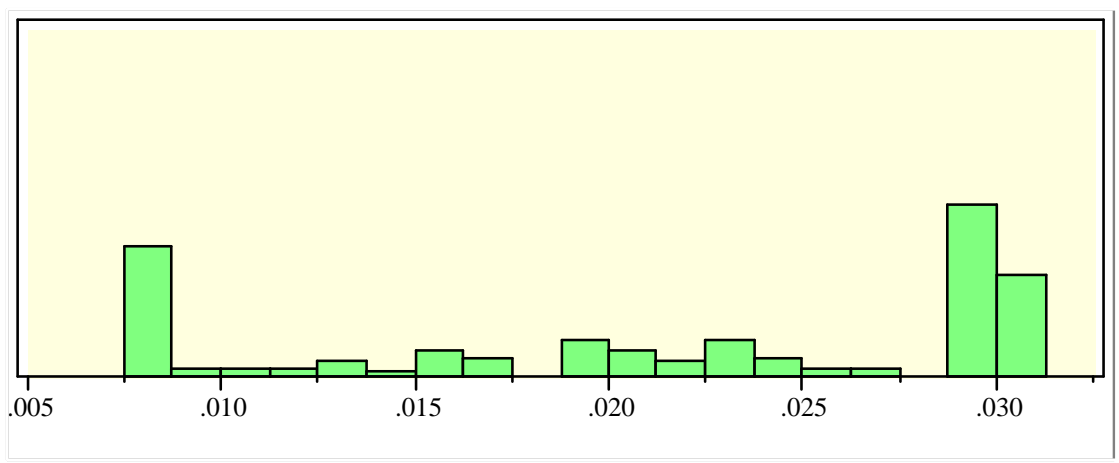

Quantiles

$\begin{array}{lll}100.0 \% & \text { maximum } & 0.03000 \\ 99.5 \% & & 0.03000 \\ 97.5 \% & & 0.03000 \\ 90.0 \% & & 0.03000 \\ 75.0 \% & \text { quartile } & 0.02999 \\ 50.0 \% & \text { median } & 0.02348 \\ 25.0 \% & \text { quartile } & 0.01263 \\ 10.0 \% & & 0.00750 \\ 2.5 \% & & 0.00750 \\ 0.5 \% & & 0.00750 \\ 0.0 \% & \text { minimum } & 0.00750\end{array}$




\section{$\mathrm{C2O4}$}

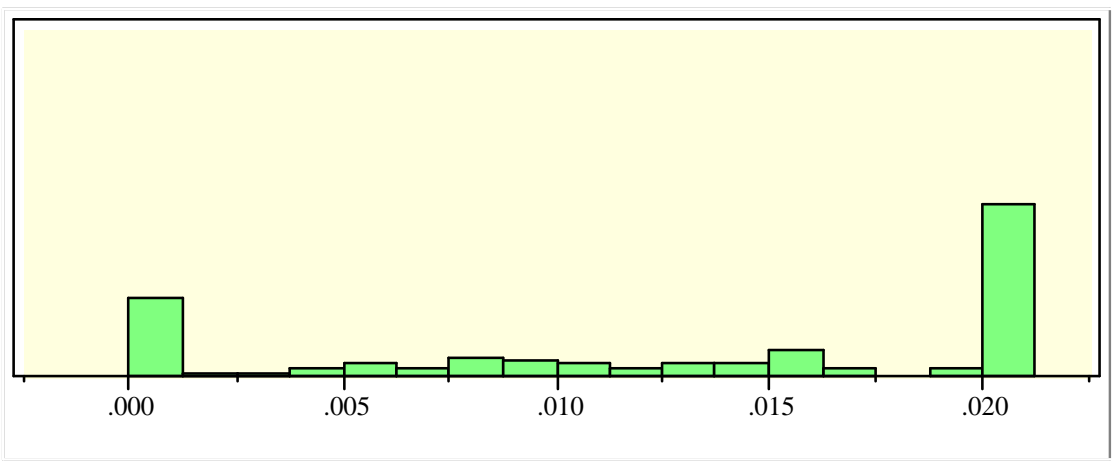

\section{Quantiles}

$\begin{array}{lll}100.0 \% & \text { maximum } & 0.02050 \\ 99.5 \% & & 0.02050 \\ 97.5 \% & & 0.02050 \\ & & 0.02050 \\ 90.0 \% & & 0.02049 \\ 75.0 \% & \text { quartile } & 0.01538 \\ 50.0 \% & \text { median } & 0.00571 \\ 25.0 \% & \text { quartile } & 0.00001 \\ 10.0 \% & & 0.00001 \\ 2.5 \% & & 0.00001 \\ 0.5 \% & & 0.00001\end{array}$


Exhibit A1: Histograms of Input Values for the Final Test Matrix

\section{(continued)}

\section{$\mathrm{CO3}$}

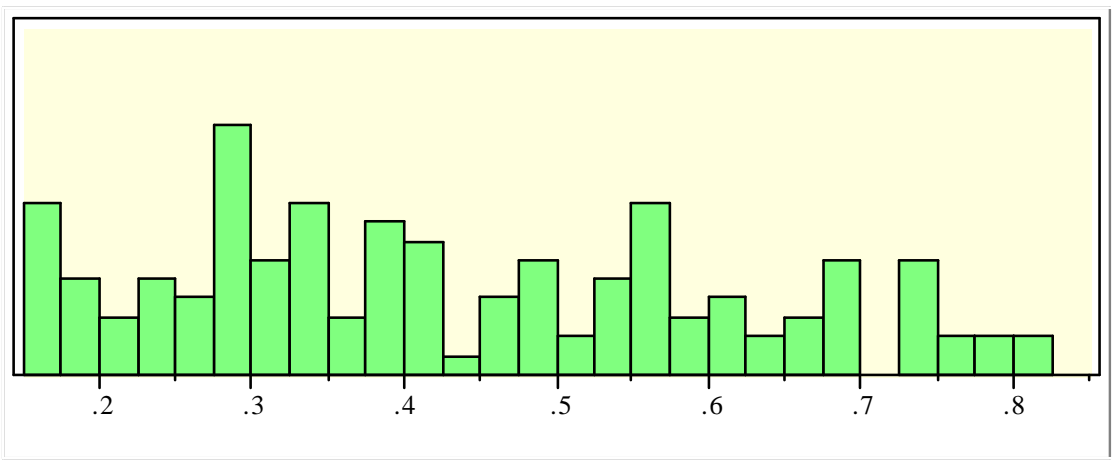

\section{Quantiles}

$\begin{array}{lll}100.0 \% & \text { maximum } & 0.80500 \\ 99.5 \% & & 0.80500 \\ & & 0.78657 \\ 97.5 \% & & 0.68989 \\ 90.0 \% & & 0.57017 \\ 75.0 \% & \text { quartile } & 0.39332 \\ 50.0 \% & \text { median } & 0.28648 \\ 25.0 \% & \text { quartile } & 0.19547 \\ 10.0 \% & & 0.16500 \\ 2.5 \% & & 0.16500 \\ 0.5 \% & & 0.16500\end{array}$




\section{NO2}

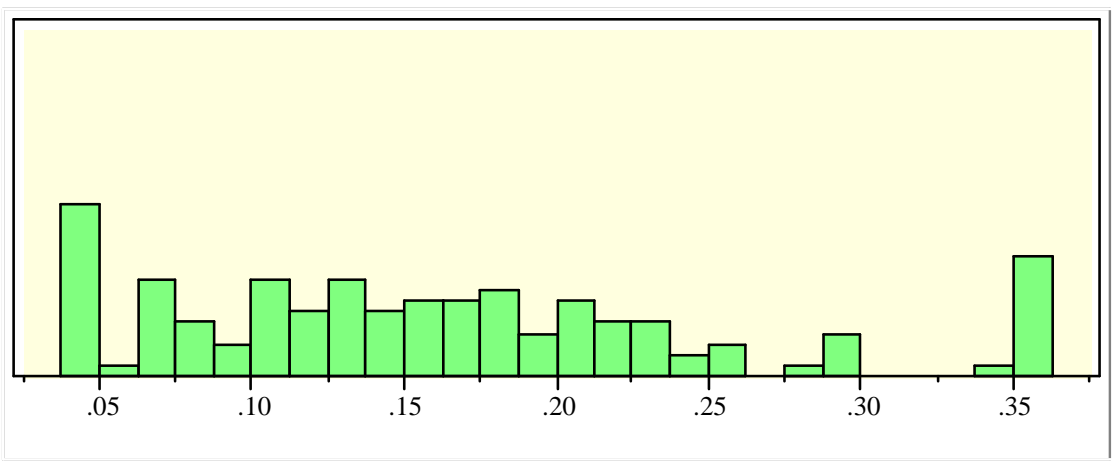

\section{Quantiles}

$\begin{array}{lll}100.0 \% & \text { maximum } & 0.36000 \\ 99.5 \% & & 0.36000 \\ 97.5 \% & & 0.36000 \\ & & 0.29981 \\ 90.0 \% & & 0.21225 \\ 75.0 \% & \text { quartile } & 0.15135 \\ 50.0 \% & \text { median } & 0.09091 \\ 25.0 \% & \text { quartile } & 0.03900 \\ 10.0 \% & & 0.03900 \\ 2.5 \% & & 0.03900 \\ 0.5 \% & & 0.03900\end{array}$


Exhibit A1: Histograms of Input Values for the Final Test Matrix

(continued)

\section{NO3}

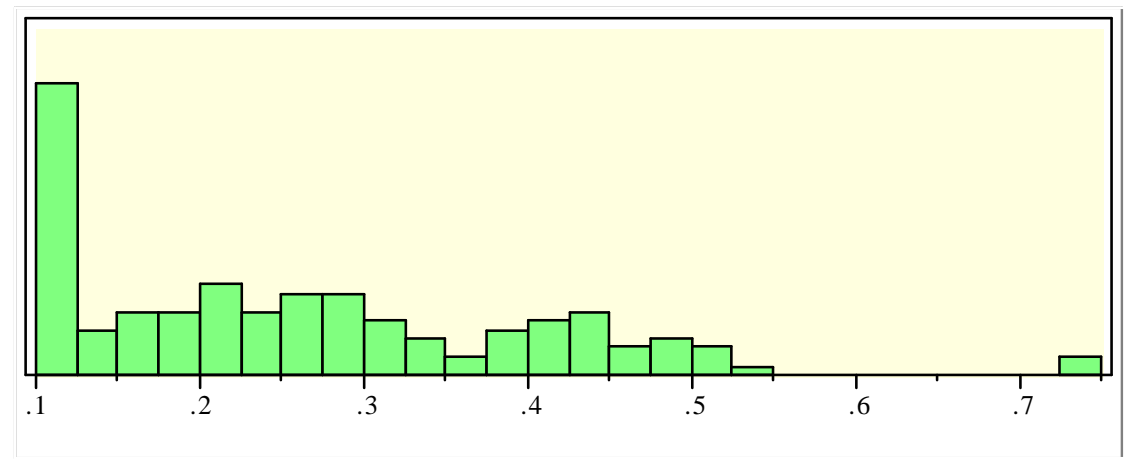

\section{Quantiles}

$\begin{array}{lll}100.0 \% & \text { maximum } & 0.73500 \\ 99.5 \% & & 0.73500 \\ & & 0.54206 \\ 97.5 \% & & 0.45544 \\ 90.0 \% & & 0.36684 \\ 75.0 \% & \text { quartile } & 0.23398 \\ 50.0 \% & \text { median } & 0.12492 \\ 25.0 \% & \text { quartile } & 0.10706 \\ 10.0 \% & & 0.10500 \\ 2.5 \% & & 0.10500 \\ 0.5 \% & & 0.10500\end{array}$




\section{OH}

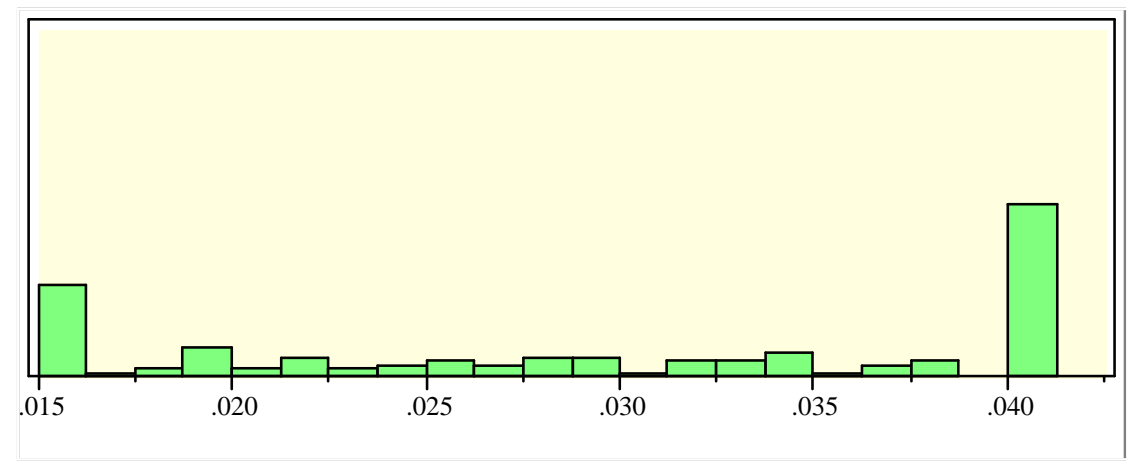

\section{Quantiles}

$\begin{array}{lll}100.0 \% & \text { maximum } & 0.04100 \\ 99.5 \% & & 0.04100 \\ 97.5 \% & & 0.04100 \\ & & 0.04100 \\ 90.0 \% & & 0.04099 \\ 75.0 \% & \text { quartile } & 0.03178 \\ 50.0 \% & \text { median } & 0.01991 \\ 25.0 \% & \text { quartile } & 0.01600 \\ 10.0 \% & & 0.01600 \\ 2.5 \% & & 0.01600 \\ 0.5 \% & & 0.01600\end{array}$


Exhibit A1: Histograms of Input Values for the Final Test Matrix

(continued)

\section{SO4}

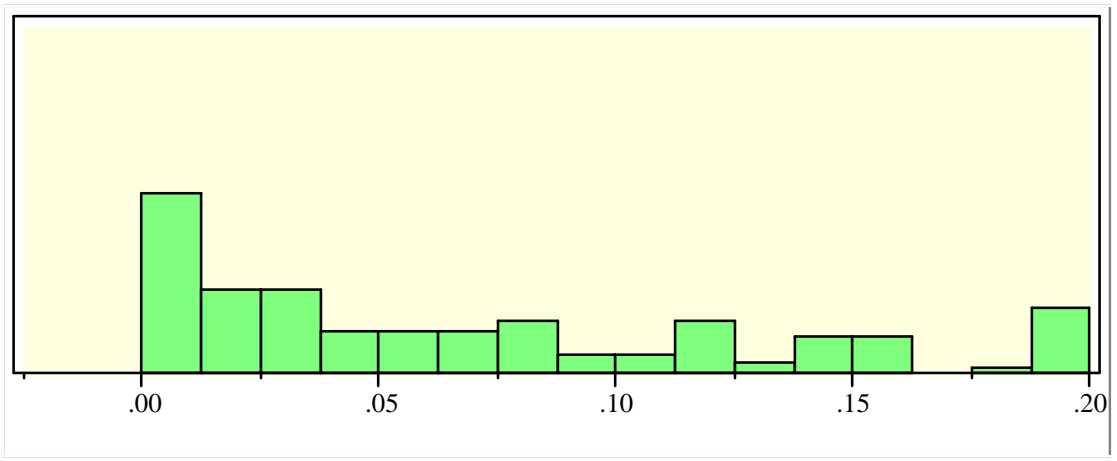

\section{Quantiles}

$\begin{array}{lll}100.0 \% & \text { maximum } & 0.19500 \\ 99.5 \% & & 0.19500 \\ & & 0.19500 \\ 97.5 \% & & 0.15639 \\ 90.0 \% & & 0.12004 \\ 75.0 \% & \text { quartile } & 0.04998 \\ 50.0 \% & \text { median } & 0.01281 \\ 25.0 \% & \text { quartile } & 0.00001 \\ 10.0 \% & & 0.00001 \\ 2.5 \% & & 0.00001 \\ 0.5 \% & & 0.00001 \\ 0.0 \% & \text { minimum } & \end{array}$




\section{TcO4}

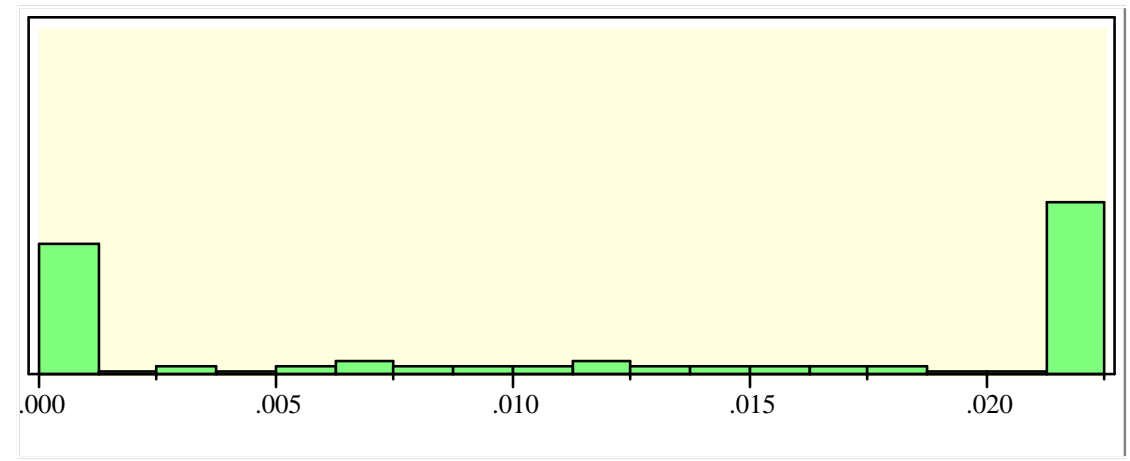

\section{Quantiles}

$\begin{array}{lll}100.0 \% & \text { maximum } & 0.02150 \\ 99.5 \% & & 0.02150 \\ 97.5 \% & & 0.02150 \\ & & 0.02150 \\ 90.0 \% & & 0.02149 \\ 75.0 \% & \text { quartile } & 0.01372 \\ 50.0 \% & \text { median } & 0.00042 \\ 25.0 \% & \text { quartile } & 0.00042 \\ 10.0 \% & & 0.00042 \\ 2.5 \% & & 0.00042 \\ 0.5 \% & & 0.00042 \\ 0.0 \% & \text { minimum } & \end{array}$


Exhibit A1: Histograms of Input Values for the Final Test Matrix

(continued)

\section{Temperature}

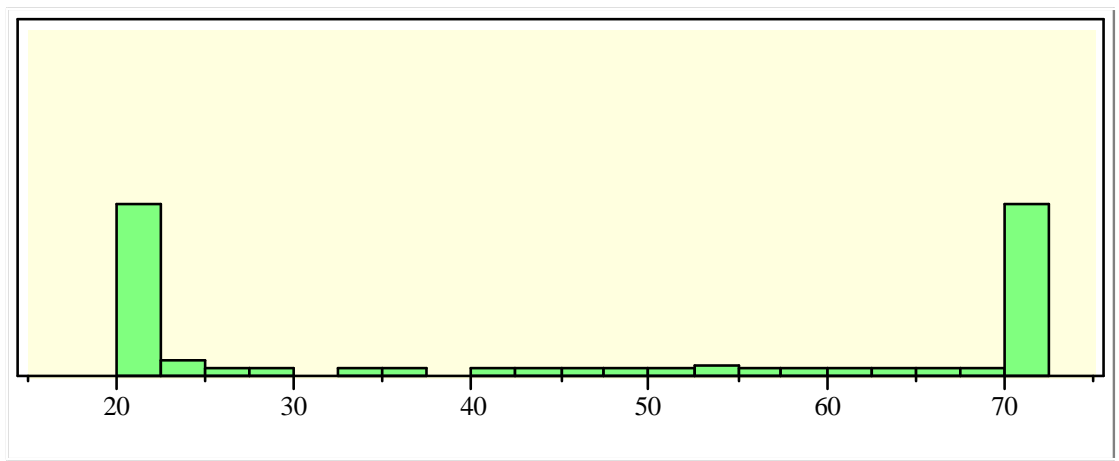

\section{Quantiles}

$\begin{array}{lll}100.0 \% & \text { maximum } & 70.000 \\ 99.5 \% & & 70.000 \\ 97.5 \% & & 70.000 \\ 90.0 \% & & 70.000 \\ 75.0 \% & \text { quartile } & 70.000 \\ 50.0 \% & \text { median } & 48.125 \\ 25.0 \% & \text { quartile } & 20.000 \\ 10.0 \% & & 20.000 \\ 2.5 \% & & 20.000 \\ 0.5 \% & & 20.000 \\ 0.0 \% & \text { minimum } & 20.000\end{array}$

